\title{
Modular Sulfondiimine Synthesis using a Stable Sulfinylamine Reagent
}

Ze-Xin Zhang, Thomas Q. Davies, and Michael C. Willis*

Department of Chemistry, Chemistry Research Laboratory, University of Oxford, Mansfield Road, Oxford, OX1 3TA, United Kingdom.

\section{Supporting information}

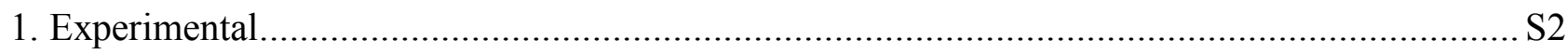

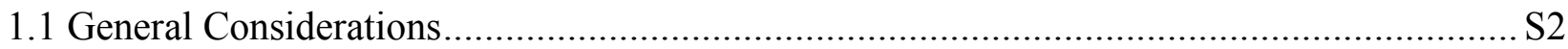

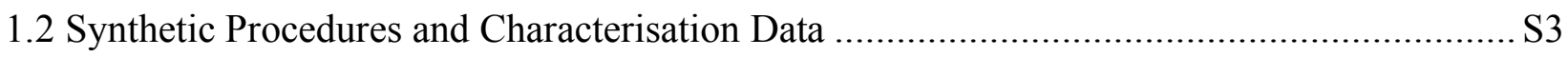

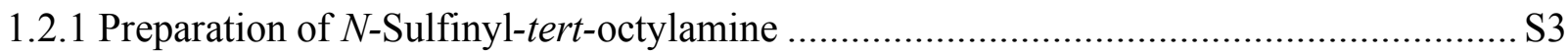

1.2.2 General Procedure A for Diaryl Sulfilimine Synthesis ............................................. S4

1.2.3 General Procedure B for Diaryl Sulfondiimine Synthesis ...................................... S14

1.2.4 General Procedure C for Aryl-Alkyl and Dialkyl Sulfondiimine Synthesis ................. S14

1.2.5 General Procedure D for Diaryl Sulfoximine Synthesis ......................................... S29

1.2.6 General Procedure E for Aryl-Alkyl and Dialkyl Sulfoximine Synthesis ................... S29

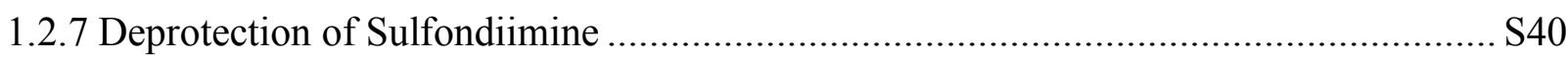

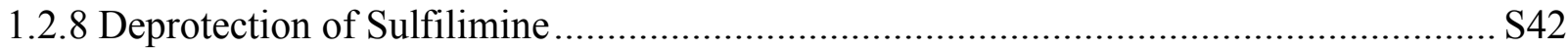

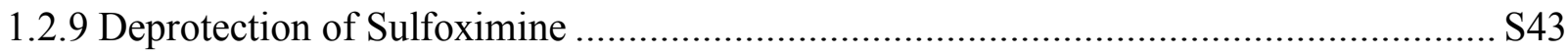

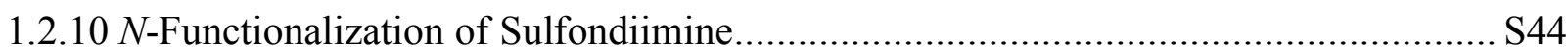

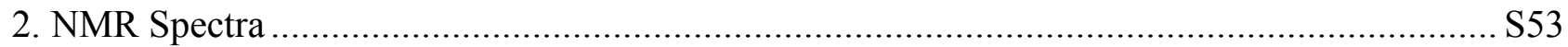

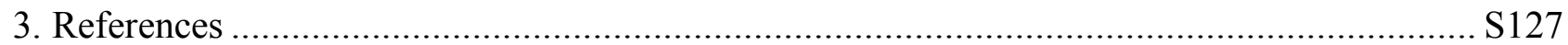




\section{Experimental}

\subsection{General Considerations}

Reactions were performed under inert nitrogen atmosphere with anhydrous solvent unless otherwise stated. All glassware was oven dried at $200{ }^{\circ} \mathrm{C}$ and allowed to cool to room temperature under positive pressure of nitrogen. Reactions were monitored by TLC until deemed complete using aluminum backed silica plates. Plates were visualised under ultraviolet light $(254 \mathrm{~nm})$ and/or by staining with $\mathrm{KMnO}_{4}$ solution. Cooling of reaction mixtures to $0{ }^{\circ} \mathrm{C}$ was achieved using an ice-water bath. Cooling of reaction mixtures between $-20{ }^{\circ} \mathrm{C}$ and $-78{ }^{\circ} \mathrm{C}$ was achieved using a dry ice-acetone bath.

Reagents were purchased from Sigma-Aldrich Chemical Co. Ltd., Alfa Aesar, Acros Organics Ltd., Fluorochem Ltd. or Strem Chemicals Inc. and were used as supplied. Grignard and organolithium reagents were titrated against salicylaldehyde phenylhydrazone. ${ }^{1}$ Flash column chromatography was carried out using matrix 60 silica gel (particle size $0.040-0.063 \mathrm{~nm}$ ). 'Petrol' refers to the fraction of light petroleum ether boiling in the range $40-60{ }^{\circ} \mathrm{C}$.

${ }^{1} \mathrm{H}-\mathrm{NMR}$ spectra were obtained on a Bruker AVIII400 (400 MHz) spectrometer using the residual solvent as an internal standard. ${ }^{13} \mathrm{C}-\mathrm{NMR}$ spectra were obtained on a Bruker AVIII400 (100 MHz) using the residual solvent as an internal standard. ${ }^{19}$ F-NMR spectra were obtained on a Bruker AVIII400 (376 MHz) spectrometer. Chemical shifts $(\delta)$ are reported in parts per million (ppm) with the multiplicities of the spectra reported as following: s, singlet; $d$, doublet; $t$, triplet; q, quartet; pent., quintet; m, multiplet; app., apparent; br., broad. Coupling constants $(J)$ were given in Hertz $(\mathrm{Hz})$.

Low resolution ESI mass spectra were recorded on a Waters LCT Premier spectrometer. High resolution mass spectrometry measurements were recorded on a Bruker Daltronics MicroTOF (ESI) spectrometer by the internal service at Chemistry Research Laboratory, University of Oxford. Samples for mass spectra were prepared as $1 \mathrm{mg} / \mathrm{mL}$ solution in MeOH (LRMS, HRMS-ESI).

Infrared spectra were recorded as thin films on a Bruker Tensor 27 FT-IR spectrometer. Melting points were determined using a Stuart Scientific Melting Point Apparatus SMP1. 


\subsection{Synthetic Procedures and Characterisation Data}

\subsubsection{Preparation of $\mathrm{N}$-Sulfinyl-tert-octylamine}

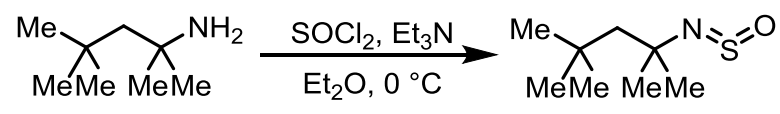

1

tert-Octylamine $(5.11 \mathrm{~g}, 39.6 \mathrm{mmol}, 1.0$ equiv.) was dissolved in anhydrous diethyl ether $(100 \mathrm{~mL})$ in a $250 \mathrm{~mL}$ 3-necked round bottom flask. Anhydrous triethylamine $(11.6 \mathrm{~mL}, 83.2 \mathrm{mmol}$, 2.1 equiv.) was added and the reaction was cooled to $0{ }^{\circ} \mathrm{C}$. Freshly distilled thionyl chloride (3.00 mL, $41.3 \mathrm{mmol}, 1.05$ equiv.) was added dropwise. The reaction was stirred at $0{ }^{\circ} \mathrm{C}$ for $2 \mathrm{~h}$. Filtration through Celite ® (washed with diethyl ether) and removal of solvent under reduced pressure at room temperature afforded $N$-sulfinyl-tert-octylamine 1 as a yellow oil $(6.71 \mathrm{~g}, 97 \%)$.

\section{Notes}

1. $\mathrm{N}$-Sulfinyl-tert-octylamine should be stored in the freezer $\left(-20^{\circ} \mathrm{C}\right)$ and can be used without loss of performance for at least 2 months.

2. CAUTION: Hydrolysis of sulfinylamines results in the formation of toxic sulfur dioxide gas. Evolution of $\mathrm{SO}_{2}$ from $N$-sulfinyl-tert-octylamine has not been observed in the normal course of use, but avoidance of contact with water or prolonged storage at room temperature is advised.

3. tert-Octylamine was purchased from Sigma-Aldrich or Fluorochem (both $£ 27$ for $100 \mathrm{~g}$ ) and used without further purification.

4. After evaporation of diethyl ether following filtration, it is advised not to redisslove the product in solvent. Doing so may result in decomposition and the formation of a solid impurity.

5. The product can be further purified by vacuum distillation at $65{ }^{\circ} \mathrm{C} / 5 \mathrm{mbar}$, but is not necessary. High temperature during distillation may cause decomposition of the product.

${ }^{\mathbf{1}} \mathbf{H}$ NMR $\left(400 \mathrm{MHz}, \mathrm{CDCl}_{3}\right): \delta(\mathrm{ppm})=1.72(\mathrm{~s}, 2 \mathrm{H}), 1.59(\mathrm{~s}, 6 \mathrm{H}), 1.01(\mathrm{~s}, 9 \mathrm{H})$.

${ }^{13} \mathbf{C ~ N M R}\left(100 \mathrm{MHz}, \mathrm{CDCl}_{3}\right): \delta(\mathrm{ppm})=67.6,55.5,32.1,31.5,31.2$.

IR (ATR): $\tilde{v}\left(\mathrm{~cm}^{-1}\right)=2980,2888,1382,1252,1152,1073,954$

HRMS $\left(\mathrm{EI}^{+}\right)$calcd. for $\mathrm{C}_{8} \mathrm{H}_{8} \mathrm{NOS}^{+}[\mathrm{M}+\mathrm{H}]^{+}:$176.1104; found: 176.1106 . 


\subsubsection{General Procedure A for Diaryl Sulfilimine Synthesis}

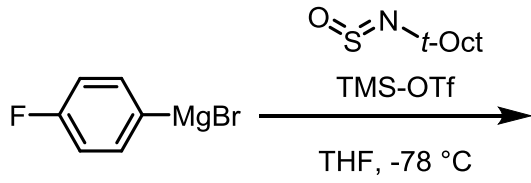

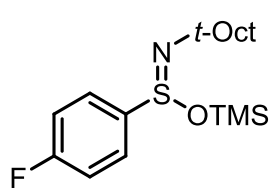

2a

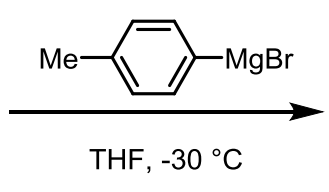

F<smiles>CCON=S(c1ccc(C)cc1)c1ccc(F)cc1</smiles>

3a

$N$-Sulfinyl-tert-octylamine 1 (158 mg, $0.90 \mathrm{mmol}, 1.05$ equiv.) was dissolved in anhydrous THF $(2 \mathrm{~mL})$ in an oven-dried $25 \mathrm{~mL}$ round bottom flask. Then the mixture was cooled to $-78{ }^{\circ} \mathrm{C}$ before TMSOTf (195 mg, $0.86 \mathrm{mmol}, 1.0$ equiv.) was added, and 4-fluorophenylmagnesium bromide ( $0.97 \mathrm{~mL}, 0.89 \mathrm{M}$ in THF, $0.86 \mathrm{mmol}, 1.0$ equiv.) was added dropwise after $1 \mathrm{~min}$. The reaction was stirred at $-78{ }^{\circ} \mathrm{C}$ for $2 \mathrm{~min}$ and then the temperature was increased to $-30{ }^{\circ} \mathrm{C}$ (see note). 4-Methylphenylmagnesium bromide (1.40 mL, 0.91 M in THF, $1.27 \mathrm{mmol}, 1.5$ equiv.) was then added quickly. The mixture was stirred at $-30{ }^{\circ} \mathrm{C}$ for $10 \mathrm{~min}$. Then the reaction was quenched with sat. aq. tetrasodium EDTA solution. Ethyl acetate $(60 \mathrm{~mL})$ was added and the organic phase was separated. The aqueous phase was further extracted with ethyl acetate $(2 \times 30 \mathrm{~mL})$. The combined organic extracts were dried over anhydrous $\mathrm{Na}_{2} \mathrm{SO}_{4}$, filtered and concentrated in vacuo. The crude product was purified by column chromatography on silica gel (petrol/ethyl acetate with $1 \% \mathrm{Et}_{3} \mathrm{~N}$, 4:1 to $1: 1$ to $0: 1)$ to afford sulfilimine $\mathbf{3 a}$ as a light yellow oil (252 $\mathrm{mg}, 85 \%)$.

Note: The reaction can be warmed from $-78{ }^{\circ} \mathrm{C}$ to $-30{ }^{\circ} \mathrm{C}$ through an addition of acetone to the dry ice-acetone bath over 5 min, or a quick replacement of the dry ice-acetone bath with an acetone bath at $-30{ }^{\circ} \mathrm{C}$.

\section{1-(4-Fluorophenyl)-1-( $p$-tolyl)- $N$-(2,4,4-trimethylpentan-2-yl)- $\lambda^{4}$-sulfanimine (3a)}<smiles>Cc1ccc([SH](N=C2OCCO2)c2ccc(F)cc2)cc1</smiles>

${ }^{1} \mathbf{H}$ NMR $\left(400 \mathrm{MHz}, \mathrm{CDCl}_{3}\right): \delta(\mathrm{ppm})=7.61-7.56(\mathrm{~m}, 2 \mathrm{H}), 7.47(\mathrm{~d}, J=8.2 \mathrm{~Hz}, 2 \mathrm{H}), 7.18(\mathrm{~d}$, $J=8.2 \mathrm{~Hz}, 2 \mathrm{H}), 7.08-7.02(\mathrm{~m}, 2 \mathrm{H}), 2.33(\mathrm{~s}, 3 \mathrm{H}), 1.63(\mathrm{~s}, 2 \mathrm{H}), 1.31(\mathrm{~s}, 6 \mathrm{H}), 0.95(\mathrm{~s}, 9 \mathrm{H})$.

${ }^{13}$ C NMR $\left(100 \mathrm{MHz}, \mathrm{CDCl}_{3}\right): \delta(\mathrm{ppm})=163.6\left(\mathrm{~d},{ }^{1} J_{\mathrm{CF}}=250.2 \mathrm{~Hz}\right), 141.8,140.9\left(\mathrm{~d},{ }^{4} J_{\mathrm{CF}}=2.6 \mathrm{~Hz}\right)$, $140.4,129.8,128.8\left(\mathrm{~d},{ }^{3} J_{\mathrm{CF}}=8.7 \mathrm{~Hz}\right), 126.7,116.1\left(\mathrm{~d},{ }^{2} J_{\mathrm{CF}}=22.2 \mathrm{~Hz}\right), 58.5,58.4,32.73,32.70,32.1$, $31.8,21.4$. 
${ }^{19} \mathbf{F}$ NMR $\left(376 \mathrm{MHz}, \mathrm{CDCl}_{3}\right): \delta(\mathrm{ppm})=-111.1(\mathrm{tt}, J=9.0,5.3 \mathrm{~Hz})$.

IR (ATR): $\tilde{v}\left(\mathrm{~cm}^{-1}\right)=2950,1587,1487,1224,1075,1007,833$.

HRMS $\left(\mathrm{ESI}^{+}\right.$) calcd. for $\mathrm{C}_{21} \mathrm{H}_{29} \mathrm{FNS}^{+}[\mathrm{M}+\mathrm{H}]^{+}$: 346.1999 ; found: 346.1997 .

\section{1-(4-Chlorophenyl)-1-(4-fluorophenyl)- $N$-(2,4,4-trimethylpentan-2-yl)- $\lambda^{4}$-sulfanimine (3g)}<smiles>CCON(C(C)=O)S(c1ccccc1)=S(c1ccc(F)cc1)c1ccc(Cl)cc1</smiles>

Prepared according to General Procedure A using $N$-sulfinyl-tert-octylamine 1 (164 mg, 0.934 mmol, 1.05 equiv.), TMSOTf (201 mg, 0.904 mmol, 1.0 equiv.), 4-chlorophenylmagnesium bromide (1.03 $\mathrm{mL}, \quad 0.88 \mathrm{M}$ in 2-methyltetrahydrofuran, $0.906 \mathrm{mmol}, 1.0$ equiv.) and 4-fluorophenylmagnesium bromide (1.42 mL, 0.96 M in THF, $1.36 \mathrm{mmol}, 1.5$ equiv.). Purification by flash column chromatography (petrol/ethyl acetate with $1 \% \mathrm{Et}_{3} \mathrm{~N}, 7: 1$ to $1: 1$ to $0: 1$ ) afforded sulfilimine $\mathbf{3 g}$ as a light yellow oil (262 $\mathrm{mg}, 79 \%)$.

${ }^{1} \mathbf{H}$ NMR $\left(400 \mathrm{MHz}, \mathrm{CDCl}_{3}\right): \delta(\mathrm{ppm})=7.63-7.57(\mathrm{~m}, 2 \mathrm{H}), 7.55(\mathrm{~d}, J=8.6 \mathrm{~Hz}, 2 \mathrm{H}), 7.35(\mathrm{~d}$, $J=8.6 \mathrm{~Hz}, 2 \mathrm{H}), 7.10-7.03(\mathrm{~m}, 2 \mathrm{H}), 1.61(\mathrm{~s}, 2 \mathrm{H}), 1.29(\mathrm{~s}, 6 \mathrm{H}), 0.94(\mathrm{~s}, 9 \mathrm{H})$.

${ }^{13}$ C NMR $\left(100 \mathrm{MHz},\left(\mathrm{CD}_{3}\right)_{2} \mathrm{CO}\right): \delta(\mathrm{ppm})=164.8\left(\mathrm{~d},{ }^{1} J_{\mathrm{CF}}=248.5 \mathrm{~Hz}\right), 146.1,142.7\left(\mathrm{~d},{ }^{4} J_{\mathrm{CF}}=\right.$ $2.9 \mathrm{~Hz}), 136.7,130.3,129.4\left(\mathrm{~d},{ }^{3} J_{\mathrm{CF}}=8.9 \mathrm{~Hz}\right), 128.6,117.2\left(\mathrm{~d},{ }^{2} J_{\mathrm{CF}}=22.9 \mathrm{~Hz}\right), 59.2,59.0,33.84$, $33.79,32.82,32.75$.

${ }^{19}$ F NMR $\left(376 \mathrm{MHz}, \mathrm{CDCl}_{3}\right): \delta(\mathrm{ppm})=-110.1(\mathrm{tt}, J=8.4,5.2 \mathrm{~Hz})$.

IR (ATR): $\tilde{v}\left(\mathrm{~cm}^{-1}\right)=1653,1589,1472,1390,1228,1128,1088,1006,963,812,736$.

HRMS $\left(\mathrm{ESI}^{+}\right.$) calcd. for $\mathrm{C}_{20} \mathrm{H}_{26} \mathrm{~F}^{35} \mathrm{Cl} \mathrm{NS}^{+}[\mathrm{M}+\mathrm{H}]^{+}:$366.1453; found: 366.1446 .

1-(4-Chlorophenyl)-1-(naphthalen-2-yl)- $N$-(2,4,4-trimethylpentan-2-yl)- $\lambda^{4}$-sulfanimine (3h)<smiles>CC(=O)N=S(c1ccc(Cl)cc1)c1ccc2ccccc2c1</smiles> 
Prepared according to General Procedure A using $N$-sulfinyl-tert-octylamine 1 (366 mg, $2.09 \mathrm{mmol}, 1.05$ equiv.), TMSOTf (445 mg, $2.00 \mathrm{mmol}, 1.0$ equiv.), 2-naphthylmagnesium bromide (3.80 mL, $0.52 \mathrm{M}$ in THF, $1.98 \mathrm{mmol}, 1.0$ equiv.) and 4-chlorophenylmagnesium bromide (3.60 mL, $0.83 \mathrm{M}$ in 2-methyltetrahydrofuran, $2.99 \mathrm{mmol}, 1.5$ equiv.). Purification by flash column chromatography (petrol/ethyl acetate with $1 \% \mathrm{Et}_{3} \mathrm{~N}, 7: 1$ to $5: 1$ to $0: 1$ ) afforded sulfilimine $\mathbf{3 h}$ as a light yellow oil (696 mg, 89\%).

${ }^{1} \mathbf{H}$ NMR $\left(400 \mathrm{MHz}, \mathrm{CDCl}_{3}\right): \delta(\mathrm{ppm})=8.30-8.27(\mathrm{~m}, 1 \mathrm{H}), 7.94-7.89(\mathrm{~m}, 1 \mathrm{H}), 7.84-7.78(\mathrm{~m}, 2 \mathrm{H})$, $7.60(\mathrm{~d}, J=8.5 \mathrm{~Hz}, 2 \mathrm{H}), 7.55-7.49(\mathrm{~m}, 3 \mathrm{H}), 7.35(\mathrm{~d}, J=8.5 \mathrm{~Hz}, 2 \mathrm{H}), 1.71(\mathrm{~s}, 2 \mathrm{H}), 1.38(\mathrm{~s}, 6 \mathrm{H}), 0.99$ $(\mathrm{s}, 9 \mathrm{H})$.

${ }^{13}$ C NMR (100 MHz, $\left.\mathrm{CDCl}_{3}\right): \delta(\mathrm{ppm})=143.6,141.7,136.1,134.0,132.9,129.4,129.2,128.7$, $128.1,127.9,127.5,127.1,126.7,123.0,58.7,58.3,32.84,32.82,32.1,31.8$.

IR (ATR): $\tilde{v}\left(\mathrm{~cm}^{-1}\right)=1474,1389,1210,1132,1092,1011,857,812,763,750$.

HRMS (ESI ${ }^{+}$) calcd. for $\mathrm{C}_{24} \mathrm{H}_{29} \mathrm{~N}^{35} \mathrm{ClS}^{+}[\mathrm{M}+\mathrm{H}]^{+}:$398.1704; found: 398.1701 .

\section{1-(4-Chlorophenyl)-1-phenyl- $N$-(2,4,4-trimethylpentan-2-yl)- $\lambda^{4}$-sulfanimine (3j)}<smiles>CCON=S(c1ccccc1)c1ccc(Cl)cc1</smiles>

Prepared according to General Procedure A using $N$-sulfinyl-tert-octylamine 1 (337 mg, $1.93 \mathrm{mmol}, 1.05$ equiv.), TMSOTf (417 mg, $1.88 \mathrm{mmol}, 1.0$ equiv.), 4-chlorophenylmagnesium bromide (2.13 mL, 0.88 M in 2-methyltetrahydrofuran, $1.87 \mathrm{mmol}, 1.0$ equiv.) and phenyllithium (2.20 mL, 1.24 $\mathrm{M}$ in dibutyl ether, $2.73 \mathrm{mmol}, 1.5$ equiv.). Purification by flash column chromatography (petrol/ethyl acetate with $1 \% \mathrm{Et}_{3} \mathrm{~N}, 3: 1$ to $1: 1$ to $0: 1$ ) afforded sulfilimine $\mathbf{3 j}$ as a light yellow oil (590 mg, 91\%).

${ }^{1} \mathbf{H}$ NMR $\left(400 \mathrm{MHz}, \mathrm{CDCl}_{3}\right): \delta(\mathrm{ppm})=7.61-7.57(\mathrm{~m}, 2 \mathrm{H}), 7.55(\mathrm{~d}, J=8.6 \mathrm{~Hz}, 2 \mathrm{H}), 7.38-7.30(\mathrm{~m}$, $5 \mathrm{H}), 1.62(\mathrm{~s}, 2 \mathrm{H}), 1.30(\mathrm{~s}, 6 \mathrm{H}), 0.94(\mathrm{~s}, 9 \mathrm{H})$.

${ }^{13} \mathrm{C}$ NMR $\left(100 \mathrm{MHz}, \mathrm{CDCl}_{3}\right): \delta(\mathrm{ppm})=144.1,143.2,136.1,130.2,129.2,129.1,128.0,126.6$, $58.6,58.1,32.6,32.5,31.9,31.7$. 
IR (ATR): $\tilde{v}\left(\mathrm{~cm}^{-1}\right)=1472,1276,1261,1209,1087,1009,817,750,702$.

HRMS (ESI ${ }^{+}$) calcd. for $\mathrm{C}_{20} \mathrm{H}_{27} \mathrm{~N}^{35} \mathrm{ClS}^{+}[\mathrm{M}+\mathrm{H}]^{+}:$348.1547; found: 348.1544 .

\section{1-(4-Chlorophenyl)-1-(6-methoxypyridin-3-yl)- $N$-(2,4,4-trimethylpentan-2-yl)- $\lambda^{4}-$ sulfanimine (3k)}<smiles>CCON(Sc1ccc(Cl)cc1)c1ccc(OC)nc1</smiles>

\section{Preparation of organolithium reagent}

5-Bromo-2-methoxypyridine (458 mg, $2.44 \mathrm{mmol}, 1.2$ equiv.) and THF (5 mL) were added to an oven-dried reaction tube and were cooled to $-78{ }^{\circ} \mathrm{C}$. $n$-Butyllithium $(1.09 \mathrm{~mL}, 2.24 \mathrm{M}$ in hexanes, 2.44 mmol, 1.2 equiv.) was added dropwise and the mixture was stirred at the same temperature for $40 \mathrm{~min}$.

\section{Preparation of diaryl sulfilimine}

$N$-Sulfinyl-tert-octylamine 1 (370 mg, $2.11 \mathrm{mmol}, 1.05$ equiv.) was dissolved in anhydrous THF $(4.0 \mathrm{~mL})$ in an oven-dried $25 \mathrm{~mL}$ round bottom flask. The mixture was cooled to $-78^{\circ} \mathrm{C}$ before the addition of TMSOTf (440 mg, $1.98 \mathrm{mmol}, 1.0$ equiv.). 4-Chlorophenylmagnesium bromide (2.25 mL, 0.88 M in 2-methyltetrahydrofuran, $1.98 \mathrm{mmol}, 1.0$ equiv.) was added dropwise after $1 \mathrm{~min}$. The reaction was stirred at $-78^{\circ} \mathrm{C}$ for $2 \mathrm{~min}$ and then the temperature was increased to $-30{ }^{\circ} \mathrm{C}$. Then the organolithium reagent was by syringe. The mixture was stirred at $-30{ }^{\circ} \mathrm{C}$ for 10 min, then warmed to room temperature and stirred for $1.5 \mathrm{~h}$. The reaction was subsequently quenched with sat. aq. tetrasodium EDTA solution. Ethyl acetate $(60 \mathrm{~mL})$ was then added and the organic phase was separated. The aqueous phase was further extracted with ethyl acetate $(2 \times 30$ $\mathrm{mL}$ ). The combined extracts were dried over anhydrous $\mathrm{Na}_{2} \mathrm{SO}_{4}$, filtered and concentrated in vacuo. The crude product was purified by flash column chromatography (petrol/ethyl acetate with $1 \% \mathrm{Et}_{3} \mathrm{~N}$, 4:1 to $1: 1$ to $0: 1)$ to afford sulfilimine $\mathbf{3 k}$ as a light yellow oil (570 $\mathrm{mg}, 76 \%)$.

${ }^{1} \mathbf{H}$ NMR $\left(400 \mathrm{MHz}, \mathrm{CDCl}_{3}\right): \delta(\mathrm{ppm})=8.26(\mathrm{dd}, J=2.5,0.7 \mathrm{~Hz}, 1 \mathrm{H}), 7.67(\mathrm{dd}, J=8.7,2.5 \mathrm{~Hz}, 1 \mathrm{H})$, $7.52(\mathrm{~d}, J=8.6 \mathrm{~Hz}, 2 \mathrm{H}), 7.31(\mathrm{~d}, J=8.6 \mathrm{~Hz}, 2 \mathrm{H}), 6.68(\mathrm{dd}, J=8.7,0.7 \mathrm{~Hz}, 1 \mathrm{H}), 3.86(\mathrm{~s}, 3 \mathrm{H}), 1.58(\mathrm{~d}$, $J=14.4 \mathrm{~Hz}, 1 \mathrm{H}), 1.54(\mathrm{~d}, J=14.4 \mathrm{~Hz}, 1 \mathrm{H}), 1.25$ (s, 3H), 1.24 (s, 3H), 0.89 (s, 9H). 
${ }^{13} \mathrm{C}$ NMR $\left(100 \mathrm{MHz}, \mathrm{CDCl}_{3}\right): \delta(\mathrm{ppm})=165.2,145.9,142.9,137.0,136.1,133.9,129.1,127.5$, $112.4,58.5,58.1,53.9,32.7,32.5,31.9,31.7$.

IR (ATR): $\tilde{v}\left(\mathrm{~cm}^{-1}\right)=1587,1473,1362,1276,1261,1010,750$.

HRMS (ESI ${ }^{+}$) calcd. for $\mathrm{C}_{20} \mathrm{H}_{28} \mathrm{O}^{35} \mathrm{Cl} \mathrm{N}_{2} \mathrm{~S}^{+}[\mathrm{M}+\mathrm{H}]^{+}: 379.1605$; found: 379.1602 .

1-(4-Chlorophenyl)-1-(thiophen-2-yl)- $N$-(2,4,4-trimethylpentan-2-yl)- $\lambda^{4}$-sulfanimine (3l)<smiles>CCON=S(c1ccc(Cl)cc1)c1cccs1</smiles>

Prepared according to General Procedure A using $N$-sulfinyl-tert-octylamine 1 (372 mg, $2.12 \mathrm{mmol}, 1.05$ equiv.), TMSOTf (458 mg, $2.06 \mathrm{mmol}, 1.0$ equiv.), 4-chlorophenylmagnesium bromide $(2.34 \mathrm{~mL}, \quad 0.88 \mathrm{M}$ in 2-methyltetrahydrofuran, $2.06 \mathrm{mmol}, 1.0$ equiv.) and 2-thienylmagnesium bromide (3.50 mL, 0.85 M in THF, $2.96 \mathrm{mmol}, 1.5$ equiv.). Purification by flash column chromatography (petrol/ethyl acetate with $1 \% \mathrm{Et}_{3} \mathrm{~N}, 7: 1$ to $2: 1$ to $0: 1$ ) afforded sulfilimine 31 as a light yellow oil (627 $\mathrm{mg}, 86 \%)$.

${ }^{1} \mathbf{H}$ NMR $\left(400 \mathrm{MHz}, \mathrm{CDCl}_{3}\right): \delta(\mathrm{ppm})=7.66(\mathrm{~d}, J=8.6 \mathrm{~Hz}, 2 \mathrm{H}), 7.43(\mathrm{dd}, J=5.0,1.3 \mathrm{~Hz}, 1 \mathrm{H}), 7.37$ $(\mathrm{d}, J=8.6 \mathrm{~Hz}, 2 \mathrm{H}), 7.27-7.21(\mathrm{~m}, 1 \mathrm{H}), 6.97(\mathrm{dd}, J=5.0,3.7 \mathrm{~Hz}, 1 \mathrm{H}), 1.63(\mathrm{~d}, J=14.5 \mathrm{~Hz}, 1 \mathrm{H}), 1.60$ $(\mathrm{d}, J=14.5 \mathrm{~Hz}, 1 \mathrm{H}), 1.32(\mathrm{~s}, 3 \mathrm{H}), 1.31(\mathrm{~s}, 3 \mathrm{H}), 0.95(\mathrm{~s}, 9 \mathrm{H})$.

${ }^{13} \mathrm{C}$ NMR $\left(100 \mathrm{MHz}, \mathrm{CDCl}_{3}\right): \delta(\mathrm{ppm})=149.4,143.0,136.4,130.6,129.1,128.8,127.4,127.2$, $58.8,57.9,32.5,32.4,31.9,31.7$.

IR (ATR): $\tilde{v}\left(\mathrm{~cm}^{-1}\right)=1471,1276,1261,1209,1087,1008,816,766,706$.

HRMS $\left(\mathrm{ESI}^{+}\right.$) calcd. for $\mathrm{C}_{18} \mathrm{H}_{25} \mathrm{~N}^{35} \mathrm{ClS}_{2}{ }^{+}[\mathrm{M}+\mathrm{H}]^{+}$: 354.1112 ; found: 354.1111 . 
<smiles>CCON=S(c1ccc(Cl)cc1)c1cc2ccccc2o1</smiles>

\section{Preparation of organolithium reagent}

Benzofuran (317 mg, $2.69 \mathrm{mmol}, 1.5$ equiv.) and THF (5.0 mL) were added to an oven-dried reaction tube. The reaction was cooled to $0{ }^{\circ} \mathrm{C}$. $n$-Butyllithium $(1.20 \mathrm{~mL}, 2.24 \mathrm{M}$ in hexanes, 2.69 mmol, 1.5 equiv.) was added dropwise and the mixture stirred at the room temperature for $1 \mathrm{~h}$.

\section{Preparation of diaryl sulfilimine}

$N$-Sulfinyl-tert-octylamine 1 (312 mg, $1.78 \mathrm{mmol}, 1.05$ equiv.) was dissolved in anhydrous THF $(3.6 \mathrm{~mL})$ in an oven-dried $25 \mathrm{~mL}$ round bottom flask. Then the reaction was cooled to $-78{ }^{\circ} \mathrm{C}$ before TMSOTf (386 mg, $1.74 \mathrm{mmol}, 1.0$ equiv.) was added, 4-chlorophenylmagnesium bromide (1.97 mL, 0.88 $\mathrm{M}$ in 2-methyltetrahydrofuran, $1.73 \mathrm{mmol}, 1.0$ equiv.) was added dropwise after $1 \mathrm{~min}$. The reaction was stirred at $-78^{\circ} \mathrm{C}$ for $2 \mathrm{~min}$ and then the temperature was increased to $-30{ }^{\circ} \mathrm{C}$. Then the lithium reagent was added by syringe. The mixture was stirred at $-30{ }^{\circ} \mathrm{C}$ for 10 min and then warmed to room temperature and stirred for $1.5 \mathrm{~h}$. Then the reaction was quenched with saturated aqueous tetrasodium EDTA solution and poured into a $250 \mathrm{~mL}$ separating funnel. Ethyl acetate $(60 \mathrm{~mL})$ was then added and the organic phase was separated. The aqueous phase was further extracted with ethyl acetate $(2 \times 30 \mathrm{~mL})$. The combined extracts were dried over anhydrous $\mathrm{Na}_{2} \mathrm{SO}_{4}$, filtered and concentrated in vacuo. The crude product was purified by flash column chromatography (petrol/ethyl acetate with $1 \% \mathrm{Et}_{3} \mathrm{~N}, 7: 1$ to $1: 1$ to $0: 1$ ) to afford sulfilimine $\mathbf{3 m}$ as a light yellow oil (584 mg, 87\%).

${ }^{1} \mathbf{H}$ NMR $\left(400 \mathrm{MHz}, \mathrm{CDCl}_{3}\right): \delta(\mathrm{ppm})=7.75(\mathrm{~d}, J=8.6 \mathrm{~Hz}, 2 \mathrm{H}), 7.57-7.52(\mathrm{~m}, 1 \mathrm{H}), 7.45-7.39(\mathrm{~m}$, 3H), 7.32-7.27 (m, 1H), 7.22 (app. td, $J=7.5,1.1 \mathrm{~Hz}, 1 \mathrm{H}), 7.04$ (d, $J=1.1 \mathrm{~Hz}, 1 \mathrm{H}), 1.68$ (s, 2H), 1.39 (s, 3H), 1.37 (s, 3H), $0.98(\mathrm{~s}, 9 \mathrm{H})$.

${ }^{13} \mathrm{C}$ NMR $\left(100 \mathrm{MHz}, \mathrm{CDCl}_{3}\right): \delta(\mathrm{ppm})=156.6,156.4,139.4,136.8,129.3,127.6,127.0,126.1$, 123.6, 122.0, 112.0, 109.8, 58.7, 57.8, 32.6, 32.4, 31.9, 31.8 .

IR (ATR): $\tilde{v}\left(\mathrm{~cm}^{-1}\right)=1472,1444,1276,1261,1010,816,750$.

HRMS $\left(\mathrm{ESI}^{+}\right)$calcd. for $\mathrm{C}_{22} \mathrm{H}_{27} \mathrm{O}^{35} \mathrm{ClNS}^{+}[\mathrm{M}+\mathrm{H}]^{+}$: 388.1496 ; found: 388.1494 . 

ethylpentan-2-yl)- $\lambda^{4}$-sulfanimine (3n)<smiles>CCON=S(c1ccc(Cl)cc1)c1ccc(-n2nc(C(F)(F)F)cc2-c2ccc(C)cc2)cc1</smiles>

\section{Preparation of organolithium reagent}

1-(4-Bromophenyl)-5-(p-tolyl)-3- (trifluoromethyl)-1H-pyrazole ${ }^{2}$ (413 mg, 1.09 mmol, 1.3 equiv.) and THF $(2.0 \mathrm{~mL})$ were added to an oven-dried reaction tube and were cooled to $-78{ }^{\circ} \mathrm{C}$. n-Butyllithium $(0.48 \mathrm{~mL}, 2.24 \mathrm{M}$ in hexanes, $1.1 \mathrm{mmol}, 1.3$ equiv.) was added dropwise and the mixture was stirred at the same temperature for $40 \mathrm{~min}$.

\section{Preparation of diaryl sulfilimine}

$N$-Sulfinyl-tert-octylamine 1 (155 mg, $0.89 \mathrm{mmol}, 1.05$ equiv.) was dissolved in anhydrous THF $(4.0 \mathrm{~mL})$ in an oven-dried $25 \mathrm{~mL}$ round bottom flask. The mixture was cooled to $-78{ }^{\circ} \mathrm{C}$ before the addition of TMSOTf (190 mg, $0.86 \mathrm{mmol}, 1.0$ equiv.). 4-Chlorophenylmagnesium bromide (0.97 mL, 0.88 $\mathrm{M}$ in 2-methyltetrahydrofuran, $0.85 \mathrm{mmol}, 1.0$ equiv.) was added dropwise after $1 \mathrm{~min}$. The reaction was stirred at $-78^{\circ} \mathrm{C}$ for $2 \mathrm{~min}$ and then the temperature was increased to $-30{ }^{\circ} \mathrm{C}$. Then the organolithium reagent was added by syringe. The mixture was stirred at $-30{ }^{\circ} \mathrm{C}$ for $10 \mathrm{~min}$, then warmed to room temperature and stirred for $1.5 \mathrm{~h}$. The reaction was subsequently quenched with sat. aq. tetrasodium EDTA solution. Ethyl acetate $(50 \mathrm{~mL})$ was then added and the organic phase was separated. The aqueous phase was further extracted with ethyl acetate $(2 \times 30$ $\mathrm{mL}$ ). The combined extracts were dried over anhydrous $\mathrm{Na}_{2} \mathrm{SO}_{4}$, filtered and concentrated in vacuo. The crude product was purified by flash column chromatography (petrol/ethyl acetate with $1 \% \mathrm{Et}_{3} \mathrm{~N}$, 3:1 to $1: 1$ to $0: 1)$ to afford sulfilimine $3 \mathbf{n}$ as a light yellow oil (413 $\mathrm{mg}, 85 \%)$.

${ }^{1} \mathbf{H}$ NMR $\left(400 \mathrm{MHz}, \mathrm{CDCl}_{3}\right): \delta(\mathrm{ppm})=7.60(\mathrm{~d}, J=8.6 \mathrm{~Hz}, 2 \mathrm{H}), 7.56(\mathrm{~d}, J=8.6 \mathrm{~Hz}, 2 \mathrm{H}), 7.38(\mathrm{~d}$, $J=8.6 \mathrm{~Hz}, 2 \mathrm{H}), 7.36(\mathrm{~d}, J=8.6 \mathrm{~Hz}, 2 \mathrm{H}), 7.11(\mathrm{~d}, J=8.3 \mathrm{~Hz}, 2 \mathrm{H}), 7.06(\mathrm{~d}, J=8.3 \mathrm{~Hz}, 2 \mathrm{H}), 6.70(\mathrm{~s}$, 1H), $2.35(\mathrm{~s}, 3 \mathrm{H}), 1.61(\mathrm{~s}, 2 \mathrm{H}), 1.32(\mathrm{~s}, 6 \mathrm{H}), 0.94(\mathrm{~s}, 9 \mathrm{H})$. 
${ }^{13}$ C NMR $\left(100 \mathrm{MHz}, \mathrm{CDCl}_{3}\right): \delta(\mathrm{ppm})=145.1,143.7\left(\mathrm{q},{ }^{2} J_{\mathrm{CF}}=38.7 \mathrm{~Hz}\right), 142.5,140.8,139.6,136.7$, $129.7,129.5,128.7,128.1,127.6,125.92,125.87,125.5,121.2\left(\mathrm{q},{ }^{1} J_{\mathrm{CF}}=268.8 \mathrm{~Hz}\right), 105.9,58.9$, $58.1,32.7,32.5,32.0,31.8,21.4$.

${ }^{19}$ F NMR $\left(376 \mathrm{MHz}, \mathrm{CDCl}_{3}\right): \delta(\mathrm{ppm})=-62.3$.

IR (ATR): $\tilde{v}\left(\mathrm{~cm}^{-1}\right)=1472,1276,1261,1235,1161,1133,1094,975,750$.

HRMS (ESI ${ }^{+}$) calcd. for $\mathrm{C}_{31} \mathrm{H}_{34}{ }^{35} \mathrm{Cl} \mathrm{N}_{3} \mathrm{~F}_{3} \mathrm{~S}^{+}[\mathrm{M}+\mathrm{H}]^{+}$: 572.2109; found: 572.2097 .

\section{1-(4-Methoxyphenyl)-1-(p-tolyl)- $N$-(2,4,4-trimethylpentan-2-yl)- $\lambda^{4}$-sulfanimine (3r)}<smiles>CCON=S(c1ccc(OC)cc1)c1ccc([N+](=O)[O-])cc1</smiles>

Prepared according to General Procedure A using $N$-sulfinyl-tert-octylamine 1 (208 mg, 1.19 mmol, 1.05 equiv.), TMSOTf (252 mg, $1.13 \mathrm{mmol}, 1.0$ equiv.), 4-methoxyphenylmagnesium bromide (2.30 mL, 0.48 M in THF, $1.10 \mathrm{mmol}, 1.0$ equiv.) and 4-methylphenylmagnesium bromide (1.80 mL, $0.94 \mathrm{M}$ in THF, $1.69 \mathrm{mmol}, 1.5$ equiv.). Purification by flash column chromatography (petrol/ethyl acetate with $1 \% \mathrm{Et}_{3} \mathrm{~N}, 3: 1$ to $1: 1$ to $0: 1$ ) afforded sulfilimine $\mathbf{3 r}$ as a light yellow oil (317 mg, 81\%).

${ }^{1} \mathbf{H}$ NMR $\left(400 \mathrm{MHz}, \mathrm{CDCl}_{3}\right): \delta(\mathrm{ppm})=7.50(\mathrm{~d}, J=8.8 \mathrm{~Hz}, 2 \mathrm{H}), 7.46(\mathrm{~d}, J=8.2 \mathrm{~Hz}, 2 \mathrm{H}), 7.15(\mathrm{~d}$, $J=8.2 \mathrm{~Hz}, 2 \mathrm{H}), 6.86(\mathrm{~d}, J=8.8 \mathrm{~Hz}, 2 \mathrm{H}), 3.74(\mathrm{~s}, 3 \mathrm{H}), 2.30(\mathrm{~s}, 3 \mathrm{H}), 1.63(\mathrm{~s}, 2 \mathrm{H}), 1.302(\mathrm{~s}, 3 \mathrm{H})$, $1.298(\mathrm{~s}, 3 \mathrm{H}), 0.94(\mathrm{~s}, 9 \mathrm{H})$.

${ }^{13} \mathbf{C}$ NMR $\left(100 \mathrm{MHz}, \mathrm{CDCl}_{3}\right): \delta(\mathrm{ppm})=160.9,142.0,139.8,136.4,129.5,128.4,126.6,114.3$, $58.3,58.2,55.4,32.62,32.56,32.0,31.7,21.2$.

IR (ATR): $\tilde{v}\left(\mathrm{~cm}^{-1}\right)=1592,1491,1392,1249,1129,1030,828,730$.

HRMS $\left(\mathrm{ESI}^{+}\right.$) calcd. for $\mathrm{C}_{22} \mathrm{H}_{32} \mathrm{NOS}^{+}[\mathrm{M}+\mathrm{H}]^{+}:$358.2199; found: 358.2200. 


\section{1-(4-Methoxyphenyl)-1-( $m$-tolyl)- $N$-(2,4,4-trimethylpentan-2-yl)- $\lambda^{4}$-sulfanimine (3s)}<smiles>COC(=O)O/N=S(/c1ccc(OC)cc1)c1cccc([N+](=O)[O-])c1</smiles>

Prepared according to General Procedure A using $N$-sulfinyl-tert-octylamine 1 (305 mg, $1.74 \mathrm{mmol}, 1.05$ equiv.), TMSOTf (369 mg, $1.66 \mathrm{mmol}, 1.0$ equiv.), 4-methoxyphenylmagnesium bromide (3.45 mL, 0.48 M in THF, $1.66 \mathrm{mmol}, 1.0$ equiv.) and 3-methylphenylmagnesium chloride (3.10 mL, $0.80 \mathrm{M}$ in THF, $2.48 \mathrm{mmol}, 1.5$ equiv.). Purification by flash column chromatography (petrol/ethyl acetate with $1 \% \mathrm{Et}_{3} \mathrm{~N}, 3: 1$ to $1: 1$ to $0: 1$ ) afforded sulfilimine $3 \mathrm{~s}$ as a light yellow oil (445 mg, 78\%).

${ }^{1} \mathbf{H}$ NMR $\left(400 \mathrm{MHz}, \mathrm{CDCl}_{3}\right): \delta(\mathrm{ppm})=7.50(\mathrm{~d}, J=8.8 \mathrm{~Hz}, 2 \mathrm{H}), 7.43$ (app. br. s, 1H), 7.35-7.30 (m, 1H), $7.21(\mathrm{t}, J=7.6 \mathrm{~Hz}, 1 \mathrm{H}), 7.13-7.08(\mathrm{~m}, 1 \mathrm{H}), 6.86(\mathrm{~d}, J=8.8 \mathrm{~Hz}, 2 \mathrm{H}), 3.74(\mathrm{~s}, 3 \mathrm{H}), 2.30(\mathrm{~s}, 3 \mathrm{H})$, $1.63(\mathrm{~s}, 2 \mathrm{H}), 1.31(\mathrm{~s}, 3 \mathrm{H}), 1.30(\mathrm{~s}, 3 \mathrm{H}), 0.93(\mathrm{~s}, 9 \mathrm{H})$.

${ }^{13}$ C NMR $\left(100 \mathrm{MHz}, \mathrm{CDCl}_{3}\right): \delta(\mathrm{ppm})=160.9,144.9,138.8,136.3,130.5,128.51,128.46,126.8$, $123.7,114.3,58.3,58.2,55.3,32.63,32.56,32.0,31.7,21.4$.

IR (ATR): $\tilde{v}\left(\mathrm{~cm}^{-1}\right)=1491,1249,827$.

HRMS (ESI ${ }^{+}$) calcd. for $\mathrm{C}_{22} \mathrm{H}_{32} \mathrm{NOS}^{+}[\mathrm{M}+\mathrm{H}]^{+}:$358.2199; found: 358.2190.

\section{1-(4-Methoxyphenyl)-1-(o-tolyl)- $N$-(2,4,4-trimethylpentan-2-yl)- $\lambda^{4}$-sulfanimine (3t)}<smiles>CCON=S(c1ccc(OC)cc1)c1ccccc1C</smiles>

Prepared according to General Procedure A using $N$-sulfinyl-tert-octylamine 1 (266 mg, $1.52 \mathrm{mmol}, 1.05$ equiv.), TMSOTf (322 mg, $1.45 \mathrm{mmol}, 1.0$ equiv.), 4-methoxyphenylmagnesium bromide (3.00 mL, $0.48 \mathrm{M}$ in THF, $1.44 \mathrm{mmol}, 1.0$ equiv.) and 2-methylphenylmagnesium chloride (3.00 mL, 0.73 M in THF, $2.19 \mathrm{mmol}, 1.5$ equiv.). Purification by flash column chromatography (petrol/ethyl acetate with $1 \% \mathrm{Et}_{3} \mathrm{~N}, 3: 1$ to $1: 1$ to $0: 1$ ) afforded sulfilimine $3 \mathrm{t}$ as a light yellow oil (400 mg, 78\%). 
${ }^{1} \mathbf{H}$ NMR $\left(400 \mathrm{MHz}, \mathrm{CDCl}_{3}\right): \delta(\mathrm{ppm})=8.10(\mathrm{dd}, J=7.8,1.5 \mathrm{~Hz}, 1 \mathrm{H}), 7.34-7.29(\mathrm{~m}, 3 \mathrm{H}), 7.23(\mathrm{td}$, $J=7.4,1.5 \mathrm{~Hz}, 1 \mathrm{H}), 7.08-7.04(\mathrm{~m}, 1 \mathrm{H}), 6.79(\mathrm{~d}, J=8.9 \mathrm{~Hz}, 2 \mathrm{H}), 3.68(\mathrm{~s}, 3 \mathrm{H}), 2.27(\mathrm{~s}, 3 \mathrm{H}), 1.63(\mathrm{~s}$, 2H), $1.28(\mathrm{~s}, 6 \mathrm{H}), 0.93(\mathrm{~s}, 9 \mathrm{H})$.

${ }^{13} \mathrm{C}$ NMR $\left(100 \mathrm{MHz}, \mathrm{CDCl}_{3}\right): \delta(\mathrm{ppm})=160.6,142.9,135.3,135.2,130.2,129.7,128.7,126.9$, $126.8,114.4,58.4,58.3,55.2,32.6,32.3,31.9,31.6,19.0$.

IR (ATR): $\tilde{v}\left(\mathrm{~cm}^{-1}\right)=1492,1250,827,756$.

HRMS $\left(\mathrm{ESI}^{+}\right)$calcd. for $\mathrm{C}_{22} \mathrm{H}_{32} \mathrm{NOS}^{+}[\mathrm{M}+\mathrm{H}]^{+}:$358.2199; found: 358.2196.

\section{1-(4-Methoxyphenyl)-1-(naphthalen-2-yl)- $N$-(2,4,4-trimethylpentan-2-yl)- $\lambda^{4}$-sulfanimine (3u)}<smiles>CCON=S(c1ccc(OC)cc1)c1ccc2ccccc2c1</smiles>

Prepared according to General Procedure A using $N$-sulfinyl-tert-octylamine 1 (358 mg, $2.04 \mathrm{mmol}, 1.05$ equiv.), TMSOTf (433 mg, $1.95 \mathrm{mmol}, 1.0$ equiv.), 4-methoxyphenylmagnesium bromide (4.05 mL, 0.48 M in THF, $1.94 \mathrm{mmol}, 1.0$ equiv.) and 2-naphthylmagnesium bromide (5.60 mL, 0.53 M in THF, $2.97 \mathrm{mmol}, 1.5$ equiv.). Purification by flash column chromatography (petrol/ethyl acetate with $1 \% \mathrm{Et}_{3} \mathrm{~N}, 3: 1$ to $1: 1$ to $0: 1$ ) afforded sulfilimine $3 \mathbf{u}$ as a light yellow oil (598 mg, 78\%).

${ }^{1} \mathbf{H}$ NMR $\left(400 \mathrm{MHz}, \mathrm{CDCl}_{3}\right): \delta(\mathrm{ppm})=8.33-8.30(\mathrm{~m}, 1 \mathrm{H}), 7.93-7.87(\mathrm{~m}, 1 \mathrm{H}), 7.81-7.74(\mathrm{~m}, 2 \mathrm{H})$, $7.57(\mathrm{~d}, J=8.8 \mathrm{~Hz}, 2 \mathrm{H}), 7.51-7.45(\mathrm{~m}, 3 \mathrm{H}), 6.87(\mathrm{~d}, J=8.8 \mathrm{~Hz}, 2 \mathrm{H}), 3.72(\mathrm{~s}, 3 \mathrm{H}), 1.73(\mathrm{~s}, 2 \mathrm{H})$, $1.40(\mathrm{~s}, 3 \mathrm{H}), 1.39(\mathrm{~s}, 3 \mathrm{H}), 0.99(\mathrm{~s}, 9 \mathrm{H})$.

${ }^{13} \mathrm{C}$ NMR $\left(100 \mathrm{MHz}, \mathrm{CDCl}_{3}\right): \delta(\mathrm{ppm})=161.0,142.2,136.0,133.8,132.9,128.8,128.6,128.5$, $127.8,127.2$, 126.8, 126.4, 123.1, 114.4, 58.5, 58.2, 55.3, 32.8, 32.6, 32.0, 31.7.

IR (ATR): $\tilde{v}\left(\mathrm{~cm}^{-1}\right)=1591,1492,1382,1249,1129,1029,825,730$.

HRMS (ESI ${ }^{+}$) calcd. for $\mathrm{C}_{25} \mathrm{H}_{32} \mathrm{NOS}^{+}[\mathrm{M}+\mathrm{H}]^{+}$: 394.2199; found: 394.2191. 


\subsubsection{General Procedure B for Diaryl Sulfondiimine Synthesis}

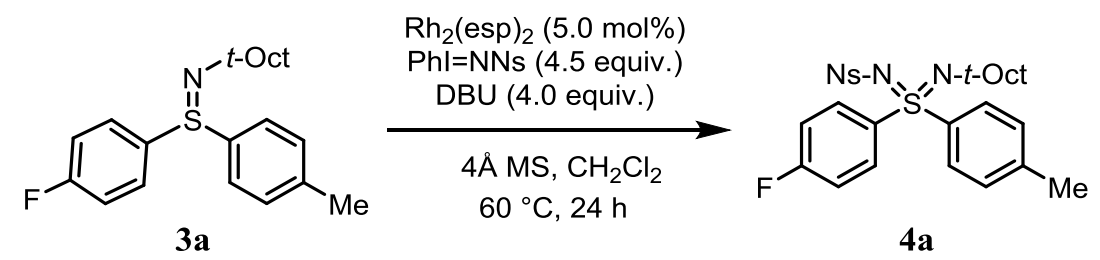

To a $10 \mathrm{~mL}$ vial containing $\mathrm{Rh}_{2}(\mathrm{esp})_{2}(9.0 \mathrm{mg}, 0.012 \mathrm{mmol}, 5.0 \mathrm{~mol} \%), 4 \AA \mathrm{MS}(c .0 .2 \mathrm{~g})$ and $\mathrm{PhI}=\mathrm{NNs}$ (136 mg, $0.337 \mathrm{mmol}, 1.5$ equiv.) was added a solution of sulfilimine 3a (80 mg, 0.23 mmol, 1.0 equiv.) in anhydrous $\mathrm{CH}_{2} \mathrm{Cl}_{2}(1.1 \mathrm{~mL})$. DBU $(0.14 \mathrm{~mL}, 0.93 \mathrm{mmol}, 4.0$ equiv. $)$ was then quickly added at room temperature and the reaction was sealed and stirred at $60{ }^{\circ} \mathrm{C}$ for $8 \mathrm{~h}$. Two portions of $\mathrm{PhI}=\mathrm{NNs}(2 \times 137 \mathrm{mg}, 0.678 \mathrm{mmol}, 3.0$ equiv. $)$ were subsequently added at the $8^{\text {th }}$ and $16^{\text {th }}$ hour. The reaction mixture was then transferred to a $100 \mathrm{~mL}$ round bottom flask, to separate from the $4 \AA \mathrm{MS}$, washing the vial several times with $\mathrm{CH}_{2} \mathrm{Cl}_{2}$. The solvent was then removed under reduced pressure. The crude product was purified by flash column chromatography (petrol/ethyl acetate 6:1 to 4:1) to afford sulfondiimine 4a as a light yellow solid (55 mg, 44\%).

\subsubsection{General Procedure C for Aryl-Alkyl and Dialkyl Sulfondiimine Synthesis}

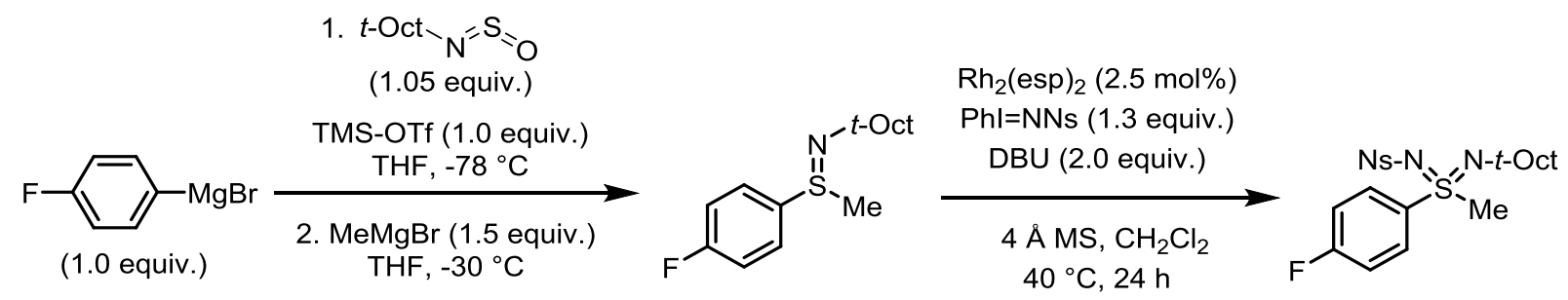

$N$-Sulfinyl-tert-octylamine 1 (240 mg, $1.37 \mathrm{mmol}, 1.05$ equiv.) was dissolved in anhydrous THF $(2.7 \mathrm{~mL})$ in an oven-dried $25 \mathrm{~mL}$ round bottom flask. The mixture was then cooled to $-78{ }^{\circ} \mathrm{C}$ and TMSOTf (297 mg, $1.34 \mathrm{mmol}, 1.0$ equiv.) was added. Then 4-fluorophenylmagnesium bromide (1.47 $\mathrm{mL}, 0.91 \mathrm{M}$ in THF, $1.34 \mathrm{mmol}, 1.0$ equiv.) was added dropwise after $1 \mathrm{~min}$. The reaction was stirred at $-78{ }^{\circ} \mathrm{C}$ for $2 \mathrm{~min}$ and then the temperature was increased to $-30{ }^{\circ} \mathrm{C}$ (see note $\mathbf{1}$ ). Methylmagnesium bromide $(0.67 \mathrm{~mL}, 3.0 \mathrm{M}$ in diethyl ether, $2.0 \mathrm{mmol}, 1.5$ equiv. $)$ was then added quickly. The mixture was stirred at $-30{ }^{\circ} \mathrm{C}$ for $10 \mathrm{~min}$. Then the reaction was quenched with sat. aq. tetrasodium EDTA solution $(100 \mathrm{~mL})$ and poured into a $250 \mathrm{~mL}$ separating funnel. Ethyl acetate $(80 \mathrm{~mL})$ was then added and the organic phase was separated. The aqueous phase was further extracted with ethyl acetate $(2 \times 40 \mathrm{~mL})$. The combined extracts were dried over anhydrous $\mathrm{Na}_{2} \mathrm{SO}_{4}$, filtered and concentrated under reduced pressure. The crude sulfilimine was dissolved in a mixture 
of diethyl ether $(15 \mathrm{~mL})$ and petroleum ether $(5 \mathrm{~mL})$ and acidified by an $1 \mathrm{M}$ aq. solution of 4-toluenesulfonic acid $(35 \mathrm{~mL})$. The organic layer was discarded. The aqueous phase was washed once with a mixture of diethyl ether $(5 \mathrm{~mL})$ and petroleum ether $(15 \mathrm{~mL})$. The aqueous phase was then extracted with $\mathrm{CH}_{2} \mathrm{Cl}_{2}(3 \times 40 \mathrm{~mL})$, combined $\mathrm{CH}_{2} \mathrm{Cl}_{2}$ extracts dried over $\mathrm{Na}_{2} \mathrm{SO}_{4}$, filtered and concentrated under reduced pressure to afford the 4-toluenesulfonic acid-sulfilimine salt 3 ba (c. $85 \%$ yield). The sulfilimine salt 3 ba was then dissolved in $\mathrm{CH}_{2} \mathrm{Cl}_{2}(50 \mathrm{~mL})$ and treated with $1 \mathrm{M}$ aq. $\mathrm{NaOH}(50 \mathrm{~mL})$. The organic layer was separated and the aqueous phase was extracted with $\mathrm{CH}_{2} \mathrm{Cl}_{2}(2 \times 50 \mathrm{~mL})$. The combined organic extracts were dried over anhydrous $\mathrm{Na}_{2} \mathrm{SO}_{4}$, filtered and concentrated under reduced pressure to afford sulfilimine $\mathbf{3 b}$ as a light yellow oil (300 $\mathrm{mg}$, $83 \%)$.

\section{Notes:}

1. The reaction can be warmed from $-78{ }^{\circ} \mathrm{C}$ to $-30{ }^{\circ} \mathrm{C}$ through an addition of acetone to the dry ice-acetone bath over $5 \mathrm{~min}$, or a quick replacement of the dry ice-acetone bath with an acetone bath at $-30{ }^{\circ} \mathrm{C}$

2. Solvents were removed in a rotary evaporator below $30{ }^{\circ} \mathrm{C}$ due to instability of the sulfilimine $\mathbf{3 b}$ at high temperatures.

3. After performing an acid-base workup as mentioned above, crude $S$-aryl-S-alkyl and $S, S$-dialkyl sulfilimines were used without further purification in the next step. For long-term storage, 4-toluenesulfonic acid salt would be preferred over the neutral sulfilimine due to enhanced stability.

4. EDTA solution is used to complex the magnesium salts present in the Grignard reagents, which otherwise emulsions may be formed and complicate the aqueous work-up if only water is used instead.

5. The $S$-aryl-S-alkyl and $S, S$-dialkyl sulfilimines are typically very polar and appear to be strongly basic, which often stay on the baseline of the TLC plate even when ethyl acetate is used as the eluent. When mixtures of ethyl acetate and methanol were used, the sulfilimine may travel further up the TLC plate but "streaking" is often observed. For sulfilimine $\mathbf{3 b}$, 3:1 petrol/ethyl acetate can be used to observe the by-products (which are removed during the diethyl ether/petroleum ether washes).

A solution of sulfilimine $\mathbf{3 b}\left(300 \mathrm{mg}, 1.11 \mathrm{mmol}, 1.0\right.$ equiv.) in anhydrous $\mathrm{CH}_{2} \mathrm{Cl}_{2}(5.6 \mathrm{~mL})$ was 
added to a $25 \mathrm{~mL}$ vial containing $\mathrm{Rh}_{2}(\mathrm{esp})_{2}(21 \mathrm{mg}, 0.028 \mathrm{mmol}, 2.5 \mathrm{~mol} \%), 4 \AA \mathrm{MS}(c .0 .4 \mathrm{~g})$ and $\mathrm{PhI}=\mathrm{NNs}$ (583 mg, $1.44 \mathrm{mmol}, 1.3$ equiv.). DBU (0.34 mL, $2.2 \mathrm{mmol}, 2.0$ equiv.) was then added at room temperature quickly and the reaction was sealed and stirred at $40{ }^{\circ} \mathrm{C}$ for $24 \mathrm{~h}$. The reaction mixture was then transferred to a $100 \mathrm{~mL}$ round bottom flask, to separate from the $4 \AA \mathrm{MS}$, washing the vial several times with $\mathrm{CH}_{2} \mathrm{Cl}_{2}$. The solvent was then removed under reduced pressure. The crude product was purified by flash column chromatography (petrol/ethyl acetate, 4:1 to $2: 1$ ) to afford sulfondiimine $\mathbf{4 b}$ as a light yellow solid (444 mg, 71\% yield over two steps).

\section{Notes:}

1. The reaction was run under air.

2. The reaction is run for 24 hours as standard as some $S, S$-diaryl substrates need extended reaction times, but it may be finished sooner for $S$-aryl-S-alkyl and $S, S$-dialkyl substrates.

3. The protected sulfondiimines are stable towards air and moisture and do not need any special care to be taken when handling them.

\section{$N$-((4-Fluorophenyl)(p-tolyl)((2,4,4-trimethylpentan-2-yl)imino)- $\lambda^{6}$-sulfaneylidene $)-4$ - nitrobenzenesulfonamide (4a)}

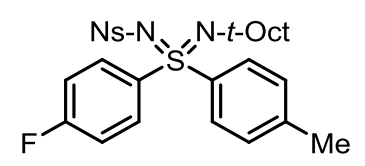

mp $169-171^{\circ} \mathrm{C}\left(\mathrm{CH}_{2} \mathrm{Cl}_{2}\right)$

$\mathbf{R}_{f} 0.50$ (petrol/ethyl acetate, 3:1).

${ }^{1} \mathbf{H}$ NMR $\left(400 \mathrm{MHz}, \mathrm{CDCl}_{3}\right): \delta(\mathrm{ppm})=8.08(\mathrm{~d}, J=8.9 \mathrm{~Hz}, 2 \mathrm{H}), 7.82-7.76(\mathrm{~m}, 2 \mathrm{H}), 7.72(\mathrm{~d}$, $J=8.9 \mathrm{~Hz}, 2 \mathrm{H}), 7.60(\mathrm{~d}, J=8.5 \mathrm{~Hz}, 2 \mathrm{H}), 7.14(\mathrm{~d}, J=8.5 \mathrm{~Hz}, 2 \mathrm{H}), 7.08-6.99(\mathrm{~m}, 2 \mathrm{H}), 2.37(\mathrm{~s}, 3 \mathrm{H})$, $1.60(\mathrm{~d}, J=14.6 \mathrm{~Hz}, 1 \mathrm{H}), 1.56(\mathrm{~d}, J=14.6 \mathrm{~Hz}, 1 \mathrm{H}), 1.38(\mathrm{~s}, 3 \mathrm{H}), 1.35(\mathrm{~s}, 3 \mathrm{H}), 1.08(\mathrm{~s}, 9 \mathrm{H})$.

${ }^{13}$ C NMR (100 MHz, $\left.\left(\mathrm{CD}_{3}\right)_{2} \mathrm{CO}\right): \delta(\mathrm{ppm})=166.0\left(\mathrm{~d},{ }^{1} J_{\mathrm{CF}}=253.5 \mathrm{~Hz}\right), 150.9,150.3,145.0,139.7$, $139.3\left(\mathrm{~d},{ }^{4} J_{\mathrm{CF}}=3.1 \mathrm{~Hz}\right), 132.2\left(\mathrm{~d},{ }^{3} J_{\mathrm{CF}}=9.5 \mathrm{~Hz}\right), 130.8,129.2,128.6,124.8,117.2(\mathrm{~d}$, $\left.{ }^{2} J_{\mathrm{CF}}=23.1 \mathrm{~Hz}\right), 61.0,58.7,32.7,32.6,32.54,32.51,21.5$.

${ }^{19}$ F NMR $\left(376 \mathrm{MHz}, \mathrm{CDCl}_{3}\right): \delta(\mathrm{ppm})=-105.2(\mathrm{tt}, J=8.0,4.9 \mathrm{~Hz})$

IR (ATR): $\tilde{v}\left(\mathrm{~cm}^{-1}\right)=2980,1528,1488,1349,1301,1222,1152,1074,1029,1005,734,612$.

HRMS $\left(\mathrm{ESI}^{+}\right)$calcd. for $\mathrm{C}_{27} \mathrm{H}_{33} \mathrm{FN}_{3} \mathrm{O}_{4} \mathrm{~S}_{2}^{+}[\mathrm{M}+\mathrm{H}]^{+}:$546.1891; found: 546.1885 . 
$N$-((4-Fluorophenyl)(methyl)((2,4,4-trimethylpentan-2-yl)imino)- $\lambda^{6}$-sulfaneylidene)-4nitrobenzenesulfonamide (4b)<smiles>N=NS(=O)(=O)c1ccc(F)cc1</smiles>

mp $153-155^{\circ} \mathrm{C}\left(\mathrm{CH}_{2} \mathrm{Cl}_{2}\right)$

$\mathbf{R}_{f} 0.54$ (petrol/ethyl acetate, 3:1).

${ }^{1} \mathbf{H}$ NMR $\left(400 \mathrm{MHz}, \mathrm{CDCl}_{3}\right): \delta(\mathrm{ppm})=8.27(\mathrm{~d}, J=8.9 \mathrm{~Hz}, 2 \mathrm{H}), 8.07(\mathrm{~d}, J=8.9 \mathrm{~Hz}, 2 \mathrm{H}), 7.99-7.94$ $(\mathrm{m}, 2 \mathrm{H}), 7.21-7.15(\mathrm{~m}, 2 \mathrm{H}), 3.46(\mathrm{~s}, 3 \mathrm{H}), 1.47(\mathrm{~d}, J=14.4 \mathrm{~Hz}, 1 \mathrm{H}), 1.41(\mathrm{~d}, J=14.4 \mathrm{~Hz}, 1 \mathrm{H}), 1.29(\mathrm{~s}$, 3H), $1.14(\mathrm{~s}, 3 \mathrm{H}), 1.00(\mathrm{~s}, 9 \mathrm{H})$.

${ }^{13} \mathrm{C}$ NMR $\left(100 \mathrm{MHz},\left(\mathrm{CD}_{3}\right)_{2} \mathrm{CO}\right): \delta(\mathrm{ppm})=166.3\left(\mathrm{~d},{ }^{1} J_{\mathrm{CF}}=253.4 \mathrm{~Hz}\right), 151.4,150.4,139.6(\mathrm{~d}$, $\left.{ }^{4} J_{\mathrm{CF}}=3.1 \mathrm{~Hz}\right), 131.7\left(\mathrm{~d},{ }^{3} J_{\mathrm{CF}}=9.6 \mathrm{~Hz}\right), 128.6,125.1,117.4\left(\mathrm{~d},{ }^{2} J_{\mathrm{CF}}=23.0 \mathrm{~Hz}\right), 60.3,58.5,49.2,32.6$, $32.5,32.4,32.3$.

${ }^{19}$ F NMR $\left(376 \mathrm{MHz}, \mathrm{CDCl}_{3}\right): \delta(\mathrm{ppm})=-104.6(\mathrm{tt}, J=8.0,4.9 \mathrm{~Hz})$.

IR $(\mathrm{ATR}): \tilde{v}\left(\mathrm{~cm}^{-1}\right)=2953,1589,1528,1487,1349,1293,1223,1149,1035,840$.

HRMS $\left(\mathrm{ESI}^{+}\right.$) calcd. for $\mathrm{C}_{21} \mathrm{H}_{29} \mathrm{FN}_{3} \mathrm{O}_{4} \mathrm{~S}_{2}{ }^{+}[\mathrm{M}+\mathrm{H}]^{+}$: 470.1578 ; found: 470.1577 .

\section{$N$-((4-Methoxyphenyl)(methyl)((2,4,4-trimethylpentan-2-yl)imino)- $\lambda^{6}$-sulfaneylidene)-4- nitrobenzenesulfonamide (4c)}<smiles>CCON=S(=O)(N=[N+]([O-])OC)c1ccc(OC)cc1</smiles>

Sulfilimine prepared according to General Procedure C using $N$-sulfinyl-tert-octylamine 1 (164 mg, 0.937 mmol, 1.05 equiv.), TMSOTf (202 mg, 0.909 mmol, 1.0 equiv.), 4-methoxyphenylmagnesium bromide (1.85 mL, $0.49 \mathrm{M}$ in THF, $0.907 \mathrm{mmol}, 1.0$ equiv.) and methylmagnesium bromide $(0.46 \mathrm{~mL}, 3.0 \mathrm{M}$ in diethyl ether, $1.4 \mathrm{mmol}, 1.5$ equiv.). Following the general purification method, sulfilimine $\mathbf{3 c}$ was generated as light yellow oil with a crude yield of $84 \%$ (215 mg). Sulfondiimine prepared using $\mathrm{Rh}_{2}(\mathrm{esp})_{2}(15 \mathrm{mg}, 0.020 \mathrm{mmol}, 2.5 \mathrm{~mol} \%$ ), PhI=NNs (400 mg, $0.990 \mathrm{mmol}, 1.3$ equiv.) and DBU (0.23 mL, $1.5 \mathrm{mmol}, 2.0$ equiv.). Purification by flash 
column chromatography (petrol/ethyl acetate, 3:1 to 1:1) afforded sulfondiimine $\mathbf{4 c}$ as a light yellow solid (279 mg, 64\% yield over two steps).

mp $117-119{ }^{\circ} \mathrm{C}\left(\mathrm{CH}_{2} \mathrm{Cl}_{2}\right)$

$\mathbf{R}_{f} 0.5$ (petrol/ethyl acetate, $\left.1: 1\right)$.

${ }^{1} \mathbf{H}$ NMR $\left(400 \mathrm{MHz}, \mathrm{CDCl}_{3}\right): \delta(\mathrm{ppm})=8.25(\mathrm{~d}, J=8.9 \mathrm{~Hz}, 2 \mathrm{H}), 8.06(\mathrm{~d}, J=8.9 \mathrm{~Hz}, 2 \mathrm{H}), 7.84(\mathrm{~d}$, $J=9.0 \mathrm{~Hz}, 2 \mathrm{H}), 6.94(\mathrm{~d}, J=9.0 \mathrm{~Hz}, 2 \mathrm{H}), 3.84(\mathrm{~s}, 3 \mathrm{H}), 3.42(\mathrm{~s}, 3 \mathrm{H}), 1.47(\mathrm{~d}, J=14.4 \mathrm{~Hz}, 1 \mathrm{H}), 1.42(\mathrm{~d}$, $J=14.4 \mathrm{~Hz}, 1 \mathrm{H}), 1.30(\mathrm{~s}, 3 \mathrm{H}), 1.16(\mathrm{~s}, 3 \mathrm{H}), 1.00(\mathrm{~s}, 9 \mathrm{H})$.

${ }^{13} \mathrm{C}$ NMR $\left(100 \mathrm{MHz}, \mathrm{CDCl}_{3}\right): \delta(\mathrm{ppm})=163.4,150.2,149.3,133.3,129.6,127.6,124.0,114.7$, $59.8,57.8,55.8,49.7,32.0,31.9,31.83,31.80$.

IR (ATR): $\tilde{v}\left(\mathrm{~cm}^{-1}\right)=2950,1591,1528,1349,1293,1176,1083,1031,855,797$.

HRMS $\left(\mathrm{ESI}^{+}\right.$) calcd. for $\mathrm{C}_{22} \mathrm{H}_{31} \mathrm{~N}_{3} \mathrm{NaO}_{5} \mathrm{~S}_{2}^{+}[\mathrm{M}+\mathrm{Na}]^{+}:$504.1597; found: 504.1596.

\section{$N$-((4-Chlorophenyl)(methyl)((2,4,4-trimethylpentan-2-yl)imino)- $\lambda^{6}$-sulfaneylidene)-4- nitrobenzenesulfonamide (4d)}<smiles></smiles>

Sulfilimine prepared according to General Procedure C using $N$-sulfinyl-tert-octylamine 1 (316 mg, 1.81 mmol, 1.05 equiv.), TMSOTf (381 mg, 1.71 mmol, 1.0 equiv.), 4-chlorophenylmagnesium bromide $(1.95 \mathrm{~mL}, 0.88 \mathrm{M}$ in 2-methyltetrahydrofuran, $1.72 \mathrm{mmol}$, 1.0 equiv.) and methylmagnesium bromide $(0.85 \mathrm{~mL}, 3.0 \mathrm{M}$ in diethyl ether, $2.6 \mathrm{mmol}, 1.5$ equiv.). Following the general purification method, sulfilimine $\mathbf{3 d}$ was generated as light yellow oil with a crude yield of $78 \%$ (380 mg). Sulfondiimine prepared using $\mathrm{Rh}_{2}(\mathrm{esp})_{2}$ (24 mg, $0.032 \mathrm{mmol}$, $2.5 \mathrm{~mol} \%$ ), $\mathrm{PhI}=\mathrm{NNs}$ (726 mg, $1.80 \mathrm{mmol}, 1.3$ equiv.) and $\mathrm{DBU}$ (0.40 mL, $2.7 \mathrm{mmol}, 2.0$ equiv.). Purification by flash column chromatography (petrol/ethyl acetate, $7: 1$ to 3:1) afforded sulfondiimine $\mathbf{4 d}$ as a white solid (546 $\mathrm{mg}, 66 \%$ yield over two steps).

$\boldsymbol{m p} 153-155^{\circ} \mathrm{C}\left(\mathrm{CH}_{2} \mathrm{Cl}_{2}\right)$

$\mathbf{R}_{f} 0.6$ (petrol $/$ ethyl acetate $\left.=2: 1\right)$. 
${ }^{1} \mathbf{H}$ NMR $\left(400 \mathrm{MHz}, \mathrm{CDCl}_{3}\right): \delta(\mathrm{ppm})=8.25(\mathrm{~d}, J=8.9 \mathrm{~Hz}, 2 \mathrm{H}), 8.05(\mathrm{~d}, J=8.9 \mathrm{~Hz}, 2 \mathrm{H}), 7.87(\mathrm{~d}$, $J=8.7 \mathrm{~Hz}, 2 \mathrm{H}), 7.45(\mathrm{~d}, J=8.7 \mathrm{~Hz}, 2 \mathrm{H}), 3.44(\mathrm{~s}, 3 \mathrm{H}), 1.45(\mathrm{~d}, J=14.4 \mathrm{~Hz}, 1 \mathrm{H}), 1.40(\mathrm{~d}, J=14.4 \mathrm{~Hz}$, $1 \mathrm{H}), 1.28(\mathrm{~s}, 3 \mathrm{H}), 1.13(\mathrm{~s}, 3 \mathrm{H}), 0.98(\mathrm{~s}, 9 \mathrm{H})$.

${ }^{13}$ C NMR $\left(100 \mathrm{MHz}, \mathrm{CDCl}_{3}\right): \delta(\mathrm{ppm})=149.8,149.3,141.1,139.8,129.7,129.0,127.5,124.0$, $60.0,57.6,49.3,31.89,31.87,31.73,31.71$.

IR (ATR): $\tilde{v}\left(\mathrm{~cm}^{-1}\right)=1527,1349,1294,1221,1149,1089,1075,1035,1006,968,855,735$.

HRMS $\left(\mathrm{ESI}^{+}\right)$calcd. for $\mathrm{C}_{21} \mathrm{H}_{28} \mathrm{~N}_{3} \mathrm{Na}^{35} \mathrm{ClO}_{4} \mathrm{~S}_{2}{ }^{+}[\mathrm{M}+\mathrm{Na}]^{+}$: 508.1102; found: 508.1104.

\section{$N$-(Methyl(naphthalen-2-yl)((2,4,4-trimethylpentan-2-yl)imino)- $\lambda^{6}$-sulfaneylidene)-4- nitrobenzenesulfonamide $(4 \mathrm{e})$}

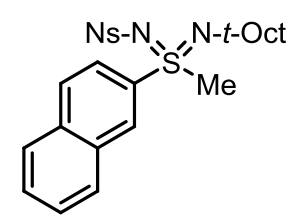

Sulfilimine prepared according to General Procedure C using $N$-sulfinyl-tert-octylamine 1 (249 mg, 1.42 mmol, 1.05 equiv.), TMSOTf (300 mg, 1.35 mmol, 1.0 equiv.), 2-naphthylmagnesium bromide $(2.60 \mathrm{~mL}, 0.52 \mathrm{M}$ in $\mathrm{THF}, 1.35 \mathrm{mmol}, 1.0$ equiv.) and methylmagnesium bromide $(0.70 \mathrm{~mL}, 3.0 \mathrm{M}$ in diethyl ether, $2.1 \mathrm{mmol}, 1.5$ equiv.). Following the general purification method, sulfilimine $\mathbf{3 e}$ was generated as light yellow oil with a crude yield of 78\% (318 mg). Sulfondiimine prepared using $\mathrm{Rh}_{2}(\mathrm{esp})_{2}(20 \mathrm{mg}, 0.026 \mathrm{mmol}, 2.5 \mathrm{~mol} \%$ ), PhI=NNs (524 mg, $1.30 \mathrm{mmol}, 1.3$ equiv.) and DBU (0.30 mL, $2.0 \mathrm{mmol}, 2.0$ equiv.). Purification by flash column chromatography (petrol/ethyl acetate, 4:1 to 2:1) afforded sulfondiimine 4e as a light yellow solid (356 mg, 53\% yield over two steps).

$\boldsymbol{m p} 154-156{ }^{\circ} \mathrm{C}\left(\mathrm{CH}_{2} \mathrm{Cl}_{2}\right)$

$\mathbf{R}_{f} 0.5$ (petrol/ethyl acetate, 1.5:1).

${ }^{1} \mathbf{H}$ NMR $\left(400 \mathrm{MHz}, \mathrm{CDCl}_{3}\right): \delta(\mathrm{ppm})=8.47(\mathrm{~d}, J=1.8 \mathrm{~Hz}, 1 \mathrm{H}), 8.16(\mathrm{~d}, J=8.9 \mathrm{~Hz}, 2 \mathrm{H}), 8.04(\mathrm{~d}$, $J=8.9 \mathrm{~Hz}, 2 \mathrm{H}), 7.96-7.84(\mathrm{~m}, 4 \mathrm{H}), 7.67-7.57(\mathrm{~m}, 2 \mathrm{H}), 3.52(\mathrm{~s}, 3 \mathrm{H}), 1.50(\mathrm{~s}, 2 \mathrm{H}), 1.36(\mathrm{~s}, 3 \mathrm{H}), 1.22(\mathrm{~s}$, $3 \mathrm{H}), 1.04(\mathrm{~s}, 9 \mathrm{H})$. 
${ }^{13} \mathrm{C}$ NMR $\left(100 \mathrm{MHz}, \mathrm{CDCl}_{3}\right): \delta(\mathrm{ppm})=149.9,149.1,138.6,134.8,132.2,129.7,129.4,129.3$, 129.2, 127.91, 127.89, 127.6, 123.9, 122.1, 60.0, 57.7, 49.2, 32.0, 31.94, 31.90, 31.8 .

IR (ATR): $\tilde{v}\left(\mathrm{~cm}^{-1}\right)=1526,1348,1293,1220,1148,1089,1031,1008,969,739$.

HRMS (ESI ${ }^{+}$) calcd. for $\mathrm{C}_{25} \mathrm{H}_{32} \mathrm{~N}_{3} \mathrm{O}_{4} \mathrm{~S}_{2}^{+}[\mathrm{M}+\mathrm{H}]^{+}:$502.1829; found: 502.1827 .

\section{$N$-(Methyl(thiophen-2-yl)((2,4,4-trimethylpentan-2-yl)imino)- $\lambda^{6}$-sulfaneylidene)-4- nitrobenzenesulfonamide (4f)}

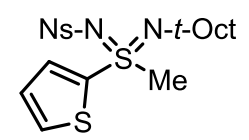

Sulfilimine prepared according to General Procedure C using $N$-sulfinyl-tert-octylamine 1 (295 mg, 1.69 mmol, 1.05 equiv.), TMSOTf (340 mg, $1.53 \mathrm{mmol}, 1.0$ equiv.), 2-thienylmagnesium bromide (1.80 mL, 0.85 M in THF, $1.53 \mathrm{mmol}, 1.0$ equiv.) and methylmagnesium bromide $(0.77$ $\mathrm{mL}, 3.0 \mathrm{M}$ in diethyl ether, $2.3 \mathrm{mmol}, 1.5$ equiv.). Following the general purification method, sulfilimine 3f was generated as light yellow oil with a crude yield of 76\% (300 mg). Sulfondiimine prepared using $\mathrm{Rh}_{2}(\mathrm{esp})_{2}$ (22 $\mathrm{mg}, 0.029 \mathrm{mmol}, 2.5 \mathrm{~mol} \%$ ), $\mathrm{PhI}=\mathrm{NNs}$ (621 mg, $1.54 \mathrm{mmol}, 1.3$ equiv.) and DBU (0.34 mL, $2.3 \mathrm{mmol}, 2.0$ equiv.). Purification by flash column chromatography (petrol/ethyl acetate, 7:1 to 3:1) afforded sulfondiimine $\mathbf{4 f}$ as a white solid (414 $\mathrm{mg}, 60 \%$ yield over two steps).

mp $132-134{ }^{\circ} \mathrm{C}\left(\mathrm{CH}_{2} \mathrm{Cl}_{2}\right)$

$\mathbf{R}_{f} 0.42$ (petrol/ethyl acetate, 3:1).

${ }^{1} \mathbf{H}$ NMR $\left(400 \mathrm{MHz}, \mathrm{CDCl}_{3}\right): \delta(\mathrm{ppm})=8.28(\mathrm{~d}, J=8.9 \mathrm{~Hz}, 2 \mathrm{H}), 8.10(\mathrm{~d}, J=8.9 \mathrm{~Hz}, 2 \mathrm{H}), 7.64(\mathrm{dd}$, $J=5.1,1.3 \mathrm{~Hz}, 1 \mathrm{H}), 7.60(\mathrm{dd}, J=3.9,1.3 \mathrm{~Hz}, 1 \mathrm{H}), 7.10(\mathrm{dd}, J=5.1,3.9 \mathrm{~Hz}, 1 \mathrm{H}), 3.58(\mathrm{~s}, 3 \mathrm{H}), 1.47$ $(\mathrm{d}, J=14.4 \mathrm{~Hz}, 1 \mathrm{H}), 1.41(\mathrm{~d}, J=14.4 \mathrm{~Hz}, 1 \mathrm{H}), 1.34$ (s, 3H), 1.19 (s, 3H), 1.00 (s, 9H).

${ }^{13} \mathbf{C}$ NMR $\left(100 \mathrm{MHz}, \mathrm{CDCl}_{3}\right): \delta(\mathrm{ppm})=149.9,149.4,144.2,134.5,132.1,128.6,127.5,124.1$, $60.3,57.6,51.1,31.9,31.8,31.6,31.5$.

IR (ATR): $\tilde{v}\left(\mathrm{~cm}^{-1}\right)=1527,1349,1293,1241,1220,1149,1090,1028,1008,854,738$.

HRMS (ESI ${ }^{+}$) calcd. for $\mathrm{C}_{19} \mathrm{H}_{27} \mathrm{~N}_{3} \mathrm{NaO}_{4} \mathrm{~S}_{3}{ }^{+}[\mathrm{M}+\mathrm{Na}]^{+}: 480.1056$; found: 480.1050 . 
$N$-((4-Chlorophenyl)(4-fluorophenyl)((2,4,4-trimethylpentan-2-yl)imino)- $\lambda^{6}$-sulfaneylidene)-4nitrobenzenesulfonamide $(\mathbf{4 g})$<smiles></smiles>

Prepared according to General Procedure B using sulfilimine 3g (152 mg, 0.416 mmol, 1.0 equiv.), $\mathrm{Rh}_{2}(\mathrm{esp})_{2}(16 \mathrm{mg}, 0.021 \mathrm{mmol}, 5.0 \mathrm{~mol} \%), \mathrm{PhI}=\mathrm{NNs}(3 \times 262 \mathrm{mg}, 1.95 \mathrm{mmol}, 4.5$ equiv. $)$ and DBU (0.25 mL, $1.7 \mathrm{mmol}, 4.0$ equiv.). Purification by flash column chromatography (petrol/ethyl acetate, 12:1 to 4:1) afforded sulfondiimine $\mathbf{4 g}$ as a white solid (111 $\mathrm{mg}, 47 \%$ yield).

mp $191-192{ }^{\circ} \mathrm{C}\left(\mathrm{CH}_{2} \mathrm{Cl}_{2}\right)$

$\mathbf{R}_{f} 0.50$ (petrol/ethyl acetate, 5:1).

${ }^{1} \mathbf{H}$ NMR $\left(400 \mathrm{MHz}, \mathrm{CDCl}_{3}\right)=8.14(\mathrm{~d}, J=8.9 \mathrm{~Hz}, 2 \mathrm{H}), 7.81-7.77(\mathrm{~m}, 2 \mathrm{H}), 7.75(\mathrm{~d}, J=8.9 \mathrm{~Hz}, 2 \mathrm{H})$, $7.69(\mathrm{~d}, J=8.8 \mathrm{~Hz}, 2 \mathrm{H}), 7.34(\mathrm{~d}, J=8.8 \mathrm{~Hz}, 2 \mathrm{H}), 7.14-6.99(\mathrm{~m}, 2 \mathrm{H}), 1.57(\mathrm{~s}, 2 \mathrm{H}), 1.35(\mathrm{~s}, 3 \mathrm{H}), 1.34$ $(\mathrm{s}, 3 \mathrm{H}), 1.07(\mathrm{~s}, 9 \mathrm{H})$.

${ }^{13}$ C NMR (100 MHz, $\left.\left(\mathrm{CD}_{3}\right)_{2} \mathrm{CO}\right): \delta(\mathrm{ppm})=166.2\left(\mathrm{~d},{ }^{1} J_{\mathrm{CF}}=253.6 \mathrm{~Hz}\right), 150.6,150.4,141.8,139.9$, $138.7\left(\mathrm{~d},{ }^{4} J_{\mathrm{CF}}=3.1 \mathrm{~Hz}\right), 132.3\left(\mathrm{~d},{ }^{4} J_{\mathrm{CF}}=9.6 \mathrm{~Hz}\right), 131.0,130.4,128.6,124.9,117.4(\mathrm{~d}$, $\left.{ }^{2} J_{\mathrm{CF}}=23.1 \mathrm{~Hz}\right), 61.2,58.6,32.6,32.52,32.48(2 \times \mathrm{C})$.

${ }^{19}$ F NMR $\left(376 \mathrm{MHz}, \mathrm{CDCl}_{3}\right): \delta(\mathrm{ppm})=-104.3(\mathrm{ddd}, J=13.0,8.0,4.9 \mathrm{~Hz})$.

IR (ATR): $\tilde{v}\left(\mathrm{~cm}^{-1}\right)=1528,1349,1299,1276,1261,1219,1152,1089,1072,1028,1002,750$.

HRMS $\left(\mathrm{ESI}^{+}\right)$calcd. for $\mathrm{C}_{26} \mathrm{H}_{29} \mathrm{~N}_{3} \mathrm{~F}^{35} \mathrm{ClNaO}_{4} \mathrm{~S}_{2}^{+}[\mathrm{M}+\mathrm{Na}]^{+}$: 588.1164; found: 588.1166.

$N$-((4-Chlorophenyl)(naphthalen-2-yl)((2,4,4-trimethylpentan-2-yl)imino)- $\lambda^{6}$-sulfaneylidene)4-nitrobenzenesulfonamide (4h)<smiles></smiles>

Prepared according to General Procedure B using sulfilimine $3 \mathbf{h}$ (253 mg, 0.637 mmol, 1.0 equiv.), $\mathrm{Rh}_{2}(\mathrm{esp})_{2}(24 \mathrm{mg}, 0.032 \mathrm{mmol}, 5.0 \mathrm{~mol} \%), \mathrm{PhI}=\mathrm{NNs}(3 \times 397 \mathrm{mg}, 2.95 \mathrm{mmol}, 4.5$ equiv. $)$ and DBU 
( $0.38 \mathrm{~mL}, 2.5 \mathrm{mmol}, 4.0$ equiv.). Purification by flash column chromatography (petrol/ethyl acetate, 7:1 to 4:1) afforded sulfondiimine $4 \mathbf{h}$ as a white solid (125 $\mathrm{mg}, 33 \%$ ).

mp $191-193{ }^{\circ} \mathrm{C}\left(\mathrm{CH}_{2} \mathrm{Cl}_{2}\right)$

$\mathbf{R}_{f} 0.50$ (petrol/ethyl acetate, 5:1).

${ }^{1} \mathbf{H}$ NMR $\left(400 \mathrm{MHz}, \mathrm{CDCl}_{3}\right)=8.33(\mathrm{~d}, J=2.3 \mathrm{~Hz}, 1 \mathrm{H}), 7.91(\mathrm{~d}, J=8.9 \mathrm{~Hz}, 2 \mathrm{H}), 7.84$ (app. d, $J=8.1 \mathrm{~Hz}, 1 \mathrm{H}), 7.81-7.73(\mathrm{~m}, 4 \mathrm{H}), 7.69-7.54(\mathrm{~m}, 5 \mathrm{H}), 7.35(\mathrm{~d}, J=8.9 \mathrm{~Hz}, 2 \mathrm{H}), 1.66(\mathrm{~d}, J=14.5 \mathrm{~Hz}$, 1H), $1.60(\mathrm{~d}, J=14.5 \mathrm{~Hz}, 1 \mathrm{H}), 1.44$ (s, 3H), 1.39 (s, 3H), 1.12 (s, 9H).

${ }^{13} \mathrm{C}$ NMR $\left(100 \mathrm{MHz}, \mathrm{CDCl}_{3}\right): \delta(\mathrm{ppm})=149.2,148.9,140.6,139.5,137.4,134.7,132.0,129.9$, $129.8,129.6,129.4,129.30,129.27,128.0,127.9,127.6,123.5,122.7,60.9,58.0,32.2,32.10,32.05$, 32.0 .

IR (ATR): $\tilde{v}\left(\mathrm{~cm}^{-1}\right)=1527,1348,1300,1276,1261,1216,1152,1088,1026,1003,909,750$.

HRMS (ESI ${ }^{+}$) calcd. for $\mathrm{C}_{30} \mathrm{H}_{33} \mathrm{~N}_{3}{ }^{35} \mathrm{ClO}_{4} \mathrm{~S}_{2}{ }^{+}[\mathrm{M}+\mathrm{H}]^{+}:$598.1596; found: 598.1592 .

\section{$N$-(Cyclopropyl(4-fluorophenyl)((2,4,4-trimethylpentan-2-yl)imino)- $\lambda^{6}$-sulfaneylidene)-4- nitrobenzenesulfonamide (4i)}

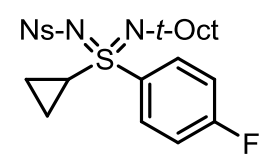

Sulfilimine prepared according to General Procedure C using $N$-sulfinyl-tert-octylamine 1 (276 mg, 1.57 mmol, 1.05 equiv.), TMSOTf (334 mg, $1.50 \mathrm{mmol}, 1.0$ equiv.), cyclopropylmagnesium bromide $(1.71 \mathrm{~mL}, 0.88 \mathrm{M}$ in 2-methyltetrahydrofuran, $1.50 \mathrm{mmol}$, 1.0 equiv.) and 4-fluorophenylmagnesium bromide $(2.30 \mathrm{~mL}, 0.96 \mathrm{M}$ in THF, $2.21 \mathrm{mmol}$, 1.5 equiv.). Following the general purification method, sulfilimine $\mathbf{3 i}$ was generated as light yellow oil with a crude yield of $65 \%(289 \mathrm{mg})$. Sulfondiimine prepared using $\mathrm{Rh}_{2}(\mathrm{esp})_{2}$ (19 $\mathrm{mg}$, $0.025 \mathrm{mmol}, 2.5 \mathrm{~mol} \%), \mathrm{PhI}=\mathrm{NNs}$ (531 mg, $1.31 \mathrm{mmol}, 1.3$ equiv.) and DBU (0.30 mL, $2.0 \mathrm{mmol}$, 2.0 equiv.). Purification by flash column chromatography (petrol/ethyl acetate, 5:1 to 3:1) afforded sulfondiimine $4 \mathbf{i}$ as a light yellow solid (297 mg, 40\% yield over two steps).

$\boldsymbol{m p} 87-89{ }^{\circ} \mathrm{C}\left(\mathrm{CH}_{2} \mathrm{Cl}_{2}\right)$

$\mathbf{R}_{f} 0.62$ (petrol/ethyl acetate, 2:1). 
${ }^{1} \mathbf{H}$ NMR $\left(400 \mathrm{MHz}, \mathrm{CDCl}_{3}\right): \delta(\mathrm{ppm})=8.26(\mathrm{~d}, J=8.9 \mathrm{~Hz}, 2 \mathrm{H}), 8.06(\mathrm{~d}, J=8.9 \mathrm{~Hz}, 2 \mathrm{H}), 7.98-7.91$ (m, 2H), 7.19-7.11 (m, 2H), $2.32(\mathrm{tt}, J=7.6,4.6 \mathrm{~Hz}, 1 \mathrm{H}), 1.71(\mathrm{ddt}, J=10.5,6.3,4.6 \mathrm{~Hz}, 1 \mathrm{H})$, 1.53-1.39 (m, 3H), 1.36 (s, 3H), 1.14 (s, 3H), 1.12-1.02 (m, 2H), 0.97 (s, 9H).

${ }^{13} \mathrm{C}$ NMR $\left(100 \mathrm{MHz},\left(\mathrm{CD}_{3}\right)_{2} \mathrm{CO}\right): \delta(\mathrm{ppm})=166.2\left(\mathrm{~d},{ }^{1} J_{\mathrm{CF}}=252.8 \mathrm{~Hz}\right), 151.9,150.3,140.3(\mathrm{~d}$, $\left.{ }^{4} J_{\mathrm{CF}}=2.8 \mathrm{~Hz}\right), 132.1\left(\mathrm{~d},{ }^{3} J_{\mathrm{CF}}=9.5 \mathrm{~Hz}\right), 128.6,125.0,117.2\left(\mathrm{~d},{ }^{2} J_{\mathrm{CF}}=22.5 \mathrm{~Hz}\right), 60.1,58.8,38.7,32.8$, $32.7,32.5,32.4,9.5,8.7$.

${ }^{19}$ F NMR $\left(376 \mathrm{MHz}, \mathrm{CDCl}_{3}\right): \delta(\mathrm{ppm})=-105.2(\mathrm{tt}, J=8.0,5.1 \mathrm{~Hz})$.

IR (ATR): $\tilde{v}\left(\mathrm{~cm}^{-1}\right)=1527,1349,1296,1276,1260,1220,1149,1090,1075,1030,1007,765,749$. HRMS $\left(\mathrm{ESI}^{+}\right)$calcd. for $\mathrm{C}_{23} \mathrm{H}_{30} \mathrm{~N}_{3} \mathrm{FNaO}_{4} \mathrm{~S}_{2}^{+}[\mathrm{M}+\mathrm{Na}]^{+}$: 518.1554; found: 518.1560 .

\section{$N$-((4-Chlorophenyl)(phenyl)((2,4,4-trimethylpentan-2-yl)imino)- $\lambda^{6}$-sulfaneylidene)-4- nitrobenzenesulfonamide $(\mathbf{4 j})$}

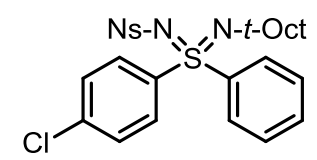

Prepared according to General Procedure B using sulfilimine 3j (251 mg, 0.723 mmol, 1.0 equiv.), $\mathrm{Rh}_{2}(\mathrm{esp})_{2}(27 \mathrm{mg}, 0.036 \mathrm{mmol}, 5.0 \mathrm{~mol} \%), \mathrm{PhI}=\mathrm{NNs}(3 \times 438 \mathrm{mg}, 3.25 \mathrm{mmol}, 4.5$ equiv. $)$ and $\mathrm{DBU}$ (0.44 mL, $2.9 \mathrm{mmol}, 4.0$ equiv.). Purification by flash column chromatography (petrol/ethyl acetate, 7:1 to 3:1) afforded sulfondiimine $\mathbf{4} \mathbf{j}$ as a light yellow solid (138 $\mathrm{mg}, 35 \%$ ).

mp $145-147^{\circ} \mathrm{C}\left(\mathrm{CH}_{2} \mathrm{Cl}_{2}\right)$

$\mathbf{R}_{f} 0.50$ (petrol/ethyl acetate $\left.=5: 1\right)$.

${ }^{1} \mathbf{H}$ NMR $\left(400 \mathrm{MHz}, \mathrm{CDCl}_{3}\right)=8.08(\mathrm{~d}, J=8.9 \mathrm{~Hz}, 2 \mathrm{H}), 7.75-7.68(\mathrm{~m}, 6 \mathrm{H}), 7.52-7.46(\mathrm{~m}, 1 \mathrm{H})$, 7.39-7.31 (m, 4H), $1.61(\mathrm{~d}, J=14.6 \mathrm{~Hz}, 1 \mathrm{H}), 1.57$ (d, $J=14.6 \mathrm{~Hz}, 1 \mathrm{H}), 1.38(\mathrm{~s}, 3 \mathrm{H}), 1.35$ (s, 3H), 1.08 $(\mathrm{s}, 9 \mathrm{H})$.

${ }^{13} \mathrm{C}$ NMR (100 MHz, $\left.\mathrm{CDCl}_{3}\right): \delta(\mathrm{ppm})=149.4,149.1,141.4,140.7,139.5,132.9,129.7,129.3$, $129.2,128.2,127.6,123.7,60.7,57.9,32.04,32.0,31.97(2 \times \mathrm{C})$.

IR (ATR): $\tilde{v}\left(\mathrm{~cm}^{-1}\right)=1527,1348,1300,1276,1261,1217,1151,1088,1071,1030,1006,995,749$. HRMS (ESI ${ }^{+}$) calcd. for $\mathrm{C}_{26} \mathrm{H}_{31} \mathrm{~N}_{3}{ }^{35} \mathrm{ClO}_{4} \mathrm{~S}_{2}{ }^{+}[\mathrm{M}+\mathrm{H}]^{+}:$548.1439; found: 548.1441. 
$N$-((4-Chlorophenyl)(6-methoxypyridin-3-yl)((2,4,4-trimethylpentan-2-yl)imino)- $\lambda^{6}-$ sulfaneylidene)-4-nitrobenzenesulfonamide (4k)<smiles>COc1ccc(S(=N)(=O)c2ccc(Cl)cc2)cn1</smiles>

Prepared according to General Procedure B using sulfilimine 3k (230 mg, 0.608 mmol, 1.0 equiv.), $\mathrm{Rh}_{2}(\mathrm{esp})_{2}(23 \mathrm{mg}, 0.030 \mathrm{mmol}, 5.0 \mathrm{~mol} \%), \mathrm{PhI}=\mathrm{NNs}(3 \times 370 \mathrm{mg}, 2.75 \mathrm{mmol}, 4.5$ equiv. $)$ and DBU ( $0.37 \mathrm{~mL}, 2.5 \mathrm{mmol}, 4.0$ equiv.). Purification by flash column chromatography (petrol/ethyl acetate, 7:1 to 3:1) afforded sulfondiimine $\mathbf{4 k}$ as a white solid (199 $\mathrm{mg}, 57 \%$ ).

mp $197-198{ }^{\circ} \mathrm{C}\left(\mathrm{CH}_{2} \mathrm{Cl}_{2}\right)$

$\mathbf{R}_{f} 0.41$ (petrol/ethyl acetate, 5:1).

${ }^{1} \mathbf{H}$ NMR $\left(400 \mathrm{MHz}, \mathrm{CDCl}_{3}\right): \delta(\mathrm{ppm})=8.46(\mathrm{~d}, J=2.4 \mathrm{~Hz}, 1 \mathrm{H}), 8.13(\mathrm{~d}, J=8.8 \mathrm{~Hz}, 2 \mathrm{H}), 7.81(\mathrm{dd}$, $J=8.9,2.4 \mathrm{~Hz}, 1 \mathrm{H}), 7.76(\mathrm{~d}, J=8.8 \mathrm{~Hz}, 2 \mathrm{H}), 7.72(\mathrm{~d}, J=8.8 \mathrm{~Hz}, 2 \mathrm{H}), 7.35(\mathrm{~d}, J=8.8 \mathrm{~Hz}, 2 \mathrm{H}), 6.68$ $(\mathrm{d}, J=8.9 \mathrm{~Hz}, 1 \mathrm{H}), 3.92(\mathrm{~s}, 3 \mathrm{H}), 1.57(\mathrm{~s}, 2 \mathrm{H}), 1.37(\mathrm{~s}, 3 \mathrm{H}), 1.35(\mathrm{~s}, 3 \mathrm{H}), 1.06(\mathrm{~s}, 9 \mathrm{H})$.

${ }^{13}$ C NMR $\left(100 \mathrm{MHz}, \mathrm{CDCl}_{3}\right): \delta(\mathrm{ppm})=166.4,149.3,149.2,148.6,140.6,139.6,137.9,130.3$, $129.46,129.45,127.6,123.8,111.5,60.8,57.9,54.5,32.03,31.99,31.95,31.93$.

IR (ATR): $\tilde{v}\left(\mathrm{~cm}^{-1}\right)=1586,1529,1477,1371,1349,1308,1276,1261,1217,1153,1087,1027$, $1000,908,750$.

HRMS (ESI ${ }^{+}$) calcd. for $\mathrm{C}_{26} \mathrm{H}_{32} \mathrm{~N}_{4}{ }^{35} \mathrm{ClO}_{5} \mathrm{~S}_{2}{ }^{+}[\mathrm{M}+\mathrm{H}]^{+}:$579.1497; found: 579.1485 .

$N$-((4-Chlorophenyl)(thiophen-2-yl)((2,4,4-trimethylpentan-2-yl)imino)- $\lambda^{6}$-sulfaneylidene)-4nitrobenzenesulfonamide (4I)

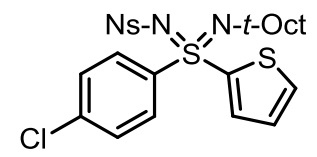

Prepared according to General Procedure B using sulfilimine 31 (203 mg, 0.575 mmol, 1.0 equiv.), $\mathrm{Rh}_{2}(\mathrm{esp})_{2}(21 \mathrm{mg}, 0.028 \mathrm{mmol}, 5.0 \mathrm{~mol} \%), \mathrm{PhI}=\mathrm{NNs}(3 \times 356 \mathrm{mg}, 2.64 \mathrm{mmol}, 4.5$ equiv. $)$ and DBU (0.35 mL, $2.3 \mathrm{mmol}, 4.0$ equiv.). Purification by flash column chromatography (petrol/ethyl acetate, 7:1 to 3:1) afforded sulfondiimine $\mathbf{4 l}$ as a light yellow solid (128 $\mathrm{mg}, 40 \%)$. 
$\boldsymbol{m p} 131-133{ }^{\circ} \mathrm{C}\left(\mathrm{CH}_{2} \mathrm{Cl}_{2}\right)$

$\mathbf{R}_{f} 0.33$ (petrol/ethyl acetate, 5:1).

${ }^{1} \mathbf{H}$ NMR $\left(400 \mathrm{MHz}, \mathrm{CDCl}_{3}\right): \delta(\mathrm{ppm})=8.15(\mathrm{~d}, J=8.8 \mathrm{~Hz}, 2 \mathrm{H}), 7.78(\mathrm{~d}, J=8.8 \mathrm{~Hz}, 2 \mathrm{H}), 7.70(\mathrm{~d}$, $J=8.9 \mathrm{~Hz}, 2 \mathrm{H}), 7.65(\mathrm{dd}, J=5.1,1.3 \mathrm{~Hz}, 1 \mathrm{H}), 7.33-7.26(\mathrm{~m}, 3 \mathrm{H}), 7.01(\mathrm{dd}, J=5.1,3.9 \mathrm{~Hz}, 1 \mathrm{H}), 1.64$ $(\mathrm{d}, J=14.5 \mathrm{~Hz}, 1 \mathrm{H}), 1.58(\mathrm{~d}, J=14.5 \mathrm{~Hz}, 1 \mathrm{H}), 1.49(\mathrm{~s}, 3 \mathrm{H}), 1.38(\mathrm{~s}, 3 \mathrm{H}), 1.08(\mathrm{~s}, 9 \mathrm{H})$.

${ }^{13}$ C NMR (100 MHz, $\left.\left(\mathrm{CD}_{3}\right)_{2} \mathrm{CO}\right): \delta(\mathrm{ppm})=150.6,150.4,144.6,142.1,139.7,136.6,134.7,130.4$, $130.2,130.0,128.5,124.9,61.4,58.6,32.63,32.56,32.5,32.1$.

$(\mathrm{ATR}): \tilde{v}\left(\mathrm{~cm}^{-1}\right)=1527,1348,1300,1276,1260,1216,1153,1089,1026,1004,749$.

HRMS $\left(\mathrm{ESI}^{+}\right.$) calcd. for $\mathrm{C}_{28} \mathrm{H}_{31} \mathrm{~N}_{3}{ }^{35} \mathrm{ClNaO}_{4} \mathrm{~S}_{3}{ }^{+}[\mathrm{M}+\mathrm{Na}]^{+}$: 576.0823 ; found: 576.0823.

\section{$N$-(Benzofuran-2-yl(4-chlorophenyl)((2,4,4-trimethylpentan-2-yl)imino)- $\lambda^{6}$-sulfaneylidene)-4- nitrobenzenesulfonamide $(4 \mathrm{~m})$}

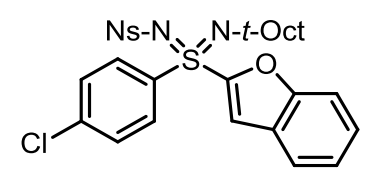

Prepared according to General Procedure B using sulfilimine 3m (240 mg, $0.620 \mathrm{mmol}$, 1.0 equiv.), $\mathrm{Rh}_{2}(\mathrm{esp})_{2}(24 \mathrm{mg}, 0.032 \mathrm{mmol}, 5.0 \mathrm{~mol} \%), \mathrm{PhI}=\mathrm{NNs}(3 \times 385 \mathrm{mg}, 2.86 \mathrm{mmol}$, 4.5 equiv.) and $\mathrm{DBU}$ (0.38 $\mathrm{mL}, 2.6 \mathrm{mmol}, 4.0$ equiv.). Purification by flash column chromatography (petrol/ethyl acetate, 7:1 to 3:1) afforded sulfondiimine $\mathbf{4 m}$ as a light yellow solid (145 mg, 40\%).

mp $201-202{ }^{\circ} \mathrm{C}\left(\mathrm{CH}_{2} \mathrm{Cl}_{2}\right)$

$\mathbf{R}_{f} 0.44$ (petrol/ethyl acetate, 5:1).

${ }^{1} \mathbf{H}$ NMR $\left(400 \mathrm{MHz}, \mathrm{CDCl}_{3}\right): \delta(\mathrm{ppm})=8.08(\mathrm{~d}, J=8.9 \mathrm{~Hz}, 2 \mathrm{H}), 7.82(\mathrm{~d}, J=8.9 \mathrm{~Hz}, 2 \mathrm{H}), 7.79(\mathrm{~d}$, $J=8.9 \mathrm{~Hz}, 2 \mathrm{H}), 7.71-7.66(\mathrm{~m}, 2 \mathrm{H}), 7.43-7.26(\mathrm{~m}, 5 \mathrm{H}), 1.63(\mathrm{~d}, J=14.6 \mathrm{~Hz}, 1 \mathrm{H}), 1.58(\mathrm{~d}, J=14.6 \mathrm{~Hz}$, 1H), 1.47 (s, 3H), 1.38 (s, 3H), 1.09 (s, 9H).

${ }^{13}$ C NMR $\left(100 \mathrm{MHz}, \mathrm{CDCl}_{3}\right): \delta(\mathrm{ppm})=156.3,149.6,149.2,149.0,140.0,137.4,129.6,129.5$, $128.2,127.7,126.1,124.7,123.6,123.2,115.6,112.2,60.9,57.5,32.2,31.92,31.87,31.1$.

IR (ATR): $\tilde{v}\left(\mathrm{~cm}^{-1}\right)=1525,1349,1301,1261,1223,1152,1077,1030,1006,833,746$. 
HRMS $\left(\mathrm{ESI}^{+}\right.$) calcd. for $\mathrm{C}_{28} \mathrm{H}_{31} \mathrm{~N}_{3}{ }^{35} \mathrm{ClO}_{5} \mathrm{~S}_{2}{ }^{+}[\mathrm{M}+\mathrm{H}]^{+}:$588.1388; found: 588.1387 .

$N$-((4-Chlorophenyl)(4-(5-(p-tolyl)-3-(trifluoromethyl)-1H-pyrazol-1-yl)phenyl)((2,4,4trimethylpentan-2-yl)imino)- $\lambda^{6}$-sulfaneylidene)-4-nitrobenzenesulfonamide (4n)<smiles>Cc1ccc(-c2cc(C(F)(F)F)nn2-c2ccc(S(=N)(=O)N=N)cc2)cc1</smiles>

Prepared according to General Procedure B using sulfilimine 3n (170 mg, 0.298 mmol, 1.0 equiv.), $\mathrm{Rh}_{2}(\mathrm{esp})_{2}(12 \mathrm{mg}, 0.016 \mathrm{mmol}, 5.0 \mathrm{~mol} \%), \mathrm{PhI}=\mathrm{NNs}(3 \times 181 \mathrm{mg}, 1.34 \mathrm{mmol}, 4.5$ equiv. $)$ and $\mathrm{DBU}$ ( $0.18 \mathrm{~mL}, 1.2 \mathrm{mmol}, 4.0$ equiv.). Purification by flash column chromatography (petrol/ethyl acetate, 7:1 to 3:1) afforded sulfondiimine $\mathbf{4 n}$ as a light blue oil (93 $\mathrm{mg}, 40 \%$ ).

$\mathbf{R}_{f} 0.56$ (petrol/ethyl acetate, 5:1).

${ }^{1} \mathbf{H}$ NMR $\left(400 \mathrm{MHz}, \mathrm{CDCl}_{3}\right): \delta(\mathrm{ppm})=8.11(\mathrm{~d}, J=8.9 \mathrm{~Hz}, 2 \mathrm{H}), 7.78(\mathrm{~d}, J=8.8 \mathrm{~Hz}, 2 \mathrm{H}), 7.75(\mathrm{~d}$, $J=8.9 \mathrm{~Hz}, 2 \mathrm{H}), 7.66(\mathrm{~d}, J=8.9 \mathrm{~Hz}, 2 \mathrm{H}), 7.39(\mathrm{~d}, J=8.8 \mathrm{~Hz}, 2 \mathrm{H}), 7.34(\mathrm{~d}, J=8.9 \mathrm{~Hz}, 2 \mathrm{H}), 7.17(\mathrm{~d}$, $J=7.9 \mathrm{~Hz}, 2 \mathrm{H}), 7.09(\mathrm{~d}, J=7.9 \mathrm{~Hz}, 2 \mathrm{H}), 6.73(\mathrm{~s}, 1 \mathrm{H}), 2.38(\mathrm{~s}, 3 \mathrm{H}), 1.61(\mathrm{~d}, J=14.5 \mathrm{~Hz}, 1 \mathrm{H}), 1.56(\mathrm{~d}$, $J=14.6 \mathrm{~Hz}, 1 \mathrm{H}), 1.38(\mathrm{~s}, 3 \mathrm{H}), 1.34(\mathrm{~s}, 3 \mathrm{H}), 1.07(\mathrm{~s}, 9 \mathrm{H})$.

${ }^{13}$ C NMR $\left(100 \mathrm{MHz},\left(\mathrm{CD}_{3}\right)_{2} \mathrm{CO}\right): \delta(\mathrm{ppm})=150.6,150.4,146.7,144.4\left(\mathrm{q},{ }^{2} J_{\mathrm{CF}}=38.2 \mathrm{~Hz}\right), 143.9$, $142.9,141.3,140.7,140.0,131.0,130.53,130.49,130.4,130.0,128.6,127.2,126.9,124.9,122.5(q$, $\left.{ }^{1} J_{\mathrm{CF}}=266.5 \mathrm{~Hz}\right), 107.0,61.4,58.6,32.63,32.59,32.5,32.4,21.5$.

${ }^{19}$ F NMR $\left(376 \mathrm{MHz}, \mathrm{CDCl}_{3}\right): \delta(\mathrm{ppm})=-62.5$

IR (ATR): $\tilde{v}\left(\mathrm{~cm}^{-1}\right)=1529,1471,1349,1276,1261,1235,1153,1090,1030,1003,827,750$.

HRMS $\left(\mathrm{ESI}^{+}\right)$calcd. for $\mathrm{C}_{37} \mathrm{H}_{38} \mathrm{~N}_{5} \mathrm{~F}_{3}{ }^{35} \mathrm{ClO}_{4} \mathrm{~S}_{2}{ }^{+}[\mathrm{M}+\mathrm{H}]^{+}:$772.2000; found: 772.1997.

\section{$N$-(Butyl(2-methylallyl)((2,4,4-trimethylpentan-2-yl)imino)- $\lambda^{6}$-sulfaneylidene)-4- nitrobenzenesulfonamide (4o)}<smiles>C=C(C)CS(CCCC)(N=O)N=[W]</smiles> 
Sulfilimine prepared according to General Procedure C using $N$-sulfinyl-tert-octylamine 1 (399 mg, 2.28 mmol, 1.05 equiv.), TMSOTf (486 mg, 2.19 mmol, 1.0 equiv.), $n$-butylmagnesium chloride (1.11 mL, 1.96 M in THF, $2.18 \mathrm{mmol}, 1.0$ equiv.) and 2-methyl-1-propenylmagnesium bromide $(6.70 \mathrm{~mL}, 0.49 \mathrm{M}$ in THF, $3.28 \mathrm{mmol}, 1.5$ equiv.). Following the general purification method, sulfilimine 3o was generated as light yellow oil with a crude yield of 77\% (454 mg). Sulfondiimine prepared using $\mathrm{Rh}_{2}(\mathrm{esp})_{2}(31 \mathrm{mg}, 0.041 \mathrm{mmol}, 2.5 \mathrm{~mol} \%$ ), $\mathrm{PhI}=\mathrm{NNs}$ (861 mg, $2.13 \mathrm{mmol}, 1.3$ equiv.) and $\mathrm{DBU}(0.50 \mathrm{~mL}, 3.4 \mathrm{mmol}, 2.0$ equiv.). Purification by flash column chromatography (petrol/ethyl acetate, 5:1 to 2:1) afforded sulfondiimine 40 as a yellow solid (163 mg, 16\% yield over two steps).

$\boldsymbol{m p} 113-115^{\circ} \mathrm{C}\left(\mathrm{CH}_{2} \mathrm{Cl}_{2}\right)$

$\mathbf{R}_{f} 0.65$ (petrol/ethyl acetate, 3:1).

${ }^{\mathbf{1}} \mathbf{H}$ NMR $\left(400 \mathrm{MHz}, \mathrm{CDCl}_{3}\right): \delta(\mathrm{ppm})=8.25(\mathrm{~d}, J=8.9 \mathrm{~Hz}, 2 \mathrm{H}), 8.04(\mathrm{~d}, J=8.9 \mathrm{~Hz}, 2 \mathrm{H}), 4.92-4.86$ (m, 2H), $4.16(\mathrm{~d}, J=19.3 \mathrm{~Hz}, 1 \mathrm{H}), 3.34$ (d, $J=19.3 \mathrm{~Hz}, 1 \mathrm{H}), 2.91-2.81(\mathrm{~m}, 1 \mathrm{H}), 2.79-2.70(\mathrm{~m}, 1 \mathrm{H})$, $2.00(\mathrm{~d}, J=14.6 \mathrm{~Hz}, 1 \mathrm{H}), 1.72(\mathrm{~s}, 3 \mathrm{H}), 1.62(\mathrm{~d}, J=14.6 \mathrm{~Hz}, 1 \mathrm{H}), 1.47$ (s, 3H), $1.30(\mathrm{~s}, 3 \mathrm{H}), 1.27-1.15$ $(\mathrm{m}, 4 \mathrm{H}), 0.97(\mathrm{~s}, 9 \mathrm{H}), 0.73-0.68(\mathrm{~m}, 3 \mathrm{H})$.

${ }^{13} \mathbf{C}$ NMR $\left(100 \mathrm{MHz}, \mathrm{CDCl}_{3}\right): \delta(\mathrm{ppm})=150.8,149.1,144.1,127.3,123.9,111.9,66.6,52.6,51.8$, $45.4,31.8,31.5,28.5,27.6,25.7,21.2,20.7,13.5$.

IR (ATR): $\tilde{v}\left(\mathrm{~cm}^{-1}\right)=1527,1348,1276,1261,1146,1091,979,910,854,750$.

HRMS (ESI ${ }^{+}$) calcd. for $\mathrm{C}_{22} \mathrm{H}_{38} \mathrm{~N}_{3} \mathrm{O}_{4} \mathrm{~S}_{2}{ }^{+}[\mathrm{M}+\mathrm{H}]^{+}:$472.2298; found: 472.2293 .

\section{$N$-(Butyl(methyl)((2,4,4-trimethylpentan-2-yl)imino)- $\lambda^{6}$-sulfaneylidene)-4- nitrobenzenesulfonamide (4p)}

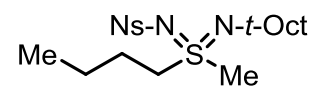

Sulfilimine prepared according to General Procedure C using $N$-sulfinyl-tert-octylamine 1 (307 mg, 1.75 mmol, 1.05 equiv.), TMSOTf (371 mg, $1.67 \mathrm{mmol}, 1.0$ equiv.), $n$-butylmagnesium chloride $(0.85 \mathrm{~mL}, 1.96 \mathrm{M}$ in THF, $1.7 \mathrm{mmol}, 1.0$ equiv.) and methylmagnesium bromide (0.84 mL, 3.0 $\mathrm{M}$ in diethyl ether, $2.5 \mathrm{mmol}, 1.5$ equiv.). Following the general purification method, sulfilimine 3p was generated as light yellow oil with a crude yield of 77\% (298 mg). Sulfondiimine prepared 
using $\mathrm{Rh}_{2}(\mathrm{esp})_{2}(22 \mathrm{mg}, 0.029 \mathrm{mmol}, 2.5 \mathrm{~mol} \%$ ), $\mathrm{PhI}=\mathrm{NNs}$ (690 mg, $1.71 \mathrm{mmol}, 1.3$ equiv.) and DBU (0.40 mL, $2.7 \mathrm{mmol}, 2.0$ equiv.). Purification by flash column chromatography (petrol/ethyl acetate, 4:1 to 2:1) afforded sulfondiimine $\mathbf{4 p}$ as a light yellow solid (187 $\mathrm{mg}, 26 \%$ yield over two steps).

$m p 80-82{ }^{\circ} \mathrm{C}\left(\mathrm{CH}_{2} \mathrm{Cl}_{2}\right)$

$\mathbf{R}_{f} 0.35$ (petrol/ethyl acetate, 2:1).

${ }^{1} \mathbf{H}$ NMR $\left(400 \mathrm{MHz}, \mathrm{CDCl}_{3}\right): \delta(\mathrm{ppm})=8.26(\mathrm{~d}, J=8.9 \mathrm{~Hz}, 2 \mathrm{H}), 8.05(\mathrm{~d}, J=8.9 \mathrm{~Hz}, 2 \mathrm{H}), 3.43-3.37$ (m, 2H), $3.17(\mathrm{~s}, 3 \mathrm{H}), 1.81-1.71(\mathrm{~m}, 2 \mathrm{H}), 1.45-1.37(\mathrm{~m}, 2 \mathrm{H}), 1.37(\mathrm{~d}, J=14.4 \mathrm{~Hz}, 1 \mathrm{H}), 1.33(\mathrm{~d}$, $J=14.4 \mathrm{~Hz}, 1 \mathrm{H}), 1.27(\mathrm{~s}, 3 \mathrm{H}), 1.23(\mathrm{~s}, 3 \mathrm{H}), 0.93-0.87(\mathrm{~m}, 12 \mathrm{H})$.

${ }^{13} \mathrm{C}$ NMR $\left(100 \mathrm{MHz}, \mathrm{CDCl}_{3}\right): \delta(\mathrm{ppm})=150.2,149.3,127.5,123.9,60.0,59.3,57.6,44.6,32.3$, $32.0,31.8,31.7,25.6,21.4,13.6$.

IR (ATR): $\tilde{v}\left(\mathrm{~cm}^{-1}\right)=1528,1349,1276,1261,1220,1146,1089,1029,1008,750$.

HRMS (ESI ${ }^{+}$) calcd. for $\mathrm{C}_{19} \mathrm{H}_{33} \mathrm{~N}_{3} \mathrm{NaO}_{4} \mathrm{~S}_{2}^{+}[\mathrm{M}+\mathrm{Na}]^{+}$: 454.1805; found: 454.1798 .

\section{$N$-(Cyclopropyl(2-methylallyl) $\left((2,4,4-t\right.$ trimethylpentan-2-yl)imino $)-\lambda^{6}$-sulfaneylidene $)-4-$ nitrobenzenesulfonamide (4q)}<smiles>C=C(C)CS(=N)(=O)NN=N</smiles>

Sulfilimine prepared according to General Procedure $\mathbf{C}$ using $N$-sulfinyl-tert-octylamine $\mathbf{1}$ (355 mg, 2.03 mmol, 1.05 equiv.), TMSOTf (444 mg, $2.00 \mathrm{mmol}, 1.0$ equiv.), cyclopropylmagnesium bromide $(2.38 \mathrm{~mL}, 0.84 \mathrm{M}$ in 2-methyltetrahydrofuran, $2.00 \mathrm{mmol}$, 1.0 equiv.) and 2-methyl-1-propenylmagnesium bromide $(6.00 \mathrm{~mL}, 0.50 \mathrm{M}$ in THF, $3.00 \mathrm{mmol}$, 1.5 equiv.). Following the general purification method, sulfilimine $\mathbf{3 q}$ was generated as light yellow oil with a crude yield of $89 \%$ (455 mg). Sulfondiimine prepared using $\mathrm{Rh}_{2}(\mathrm{esp})_{2}$ (33 $\mathrm{mg}$, $0.044 \mathrm{mmol}, 2.5 \mathrm{~mol} \%), \mathrm{PhI}=\mathrm{NNs}$ (930 mg, $2.30 \mathrm{mmol}, 1.3$ equiv.) and DBU (0.53 mL, $3.6 \mathrm{mmol}$, 2.0 equiv.). Purification by flash column chromatography (petrol/ethyl acetate, $4: 1$ to 2:1) afforded sulfondiimine $\mathbf{4 q}$ as a light brown solid (315 mg, 35\% yield over two steps).

$\boldsymbol{m p} 119-121^{\circ} \mathrm{C}\left(\mathrm{CH}_{2} \mathrm{Cl}_{2}\right)$ 
$\mathbf{R}_{f} 0.54$ (petrol/ethyl acetate, 3:1).

${ }^{1} \mathbf{H}$ NMR $\left(400 \mathrm{MHz}, \mathrm{CDCl}_{3}\right): \delta(\mathrm{ppm})=8.24(\mathrm{~d}, J=8.9 \mathrm{~Hz}, 2 \mathrm{H}), 7.99(\mathrm{~d}, J=8.9 \mathrm{~Hz}, 2 \mathrm{H}), 4.93(\mathrm{~s}$, 1H), $4.86(\mathrm{~s}, 1 \mathrm{H}), 4.08(\mathrm{~d}, J=19.3 \mathrm{~Hz}, 1 \mathrm{H}), 3.37(\mathrm{~d}, J=19.3 \mathrm{~Hz}, 1 \mathrm{H}), 2.20(\mathrm{tt}, J=7.7,4.8 \mathrm{~Hz}, 1 \mathrm{H})$, $1.97(\mathrm{~d}, J=14.6 \mathrm{~Hz}, 1 \mathrm{H}), 1.69$ (s, 3H), 1.63 (d, $J=14.6 \mathrm{~Hz}, 1 \mathrm{H}), 1.49$ (s, 3H), 1.29 (s, 3H), 1.23-1.16 (m, 1H), $0.96(\mathrm{~s}, 9 \mathrm{H}), 0.76-0.61(\mathrm{~m}, 2 \mathrm{H}), 0.27-0.21(\mathrm{~m}, 1 \mathrm{H})$.

${ }^{13} \mathrm{C}$ NMR (100 MHz, $\left.\mathrm{CDCl}_{3}\right): \delta(\mathrm{ppm})=150.8,149.0,143.3,127.2,123.9,111.2,66.4,51.8,46.1$, $31.7,31.5,29.6,28.5,27.5,20.6,3.9,2.4$.

IR (ATR): $\tilde{v}\left(\mathrm{~cm}^{-1}\right)=1526,1348,1289,1145,1090,1016,973,854,768,733$.

HRMS (ESI ${ }^{+}$) calcd. for $\mathrm{C}_{21} \mathrm{H}_{34} \mathrm{~N}_{3} \mathrm{O}_{4} \mathrm{~S}_{2}{ }^{+}[\mathrm{M}+\mathrm{H}]^{+}: 456.1985$; found: 456.1979 .

\subsubsection{General Procedure D for Diaryl Sulfoximine Synthesis}<smiles>CCON=S(c1ccc(C)cc1)c1ccc(F)cc1</smiles>

$3 \mathbf{a}$

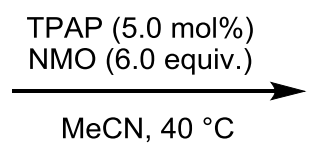

$\mathrm{MeCN}, 40^{\circ} \mathrm{C}$<smiles>CCON=S(=O)(c1ccc(C)cc1)c1ccc(F)cc1</smiles>

5d

Sulfilimine 3a (471 mg, $1.37 \mathrm{mmol}, 1.0$ equiv.) was dissolved in anhydrous $\mathrm{MeCN}(7.0 \mathrm{~mL})$ in an oven-dried $50 \mathrm{~mL}$ round bottom flask. Then the reaction was heated to $40{ }^{\circ} \mathrm{C}$ before TPAP $(24 \mathrm{mg}$, $0.068 \mathrm{mmol}, 5.0 \mathrm{~mol} \%$ ) was added, then NMO (1.01 g, $8.63 \mathrm{mmol}, 6.0$ equiv.) was added. The reaction was stirred at $40{ }^{\circ} \mathrm{C}$ for $24 \mathrm{~h}$ until completion of the reaction (TLC). The reaction was quenched with water and extracted with ethyl acetate $(3 \times 50 \mathrm{~mL})$. The combined organic extracts were washed with brine, dried over anhydrous $\mathrm{Na}_{2} \mathrm{SO}_{4}$, and concentrated. The crude product was purified by flash column chromatography (petrol/ethyl acetate, 20:1 to 10:1) to afford sulfoximine 5d as a colourless oil (490 mg, 99\%).

\subsubsection{General Procedure E for Aryl-Alkyl and Dialkyl Sulfoximine Synthesis}

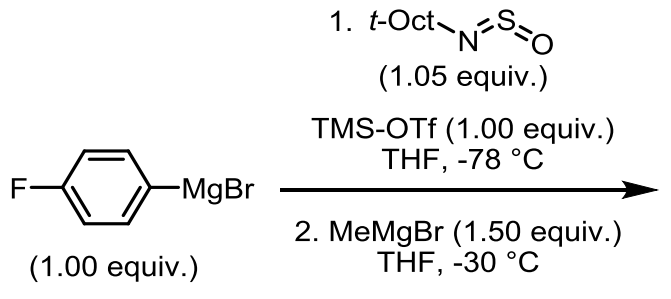

(1.00 equiv.)

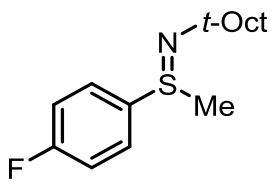

3b

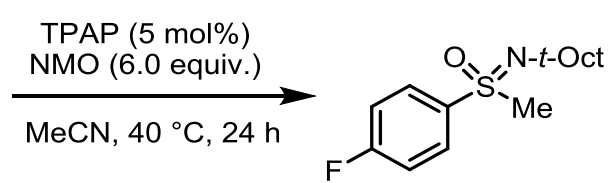

$5 a$ 
$N$-Sulfinyl-tert-octylamine 1 (248 mg, $1.42 \mathrm{mmol}, 1.05$ equiv.) was dissolved in anhydrous THF $(3.0 \mathrm{~mL})$ in an oven-dried $25 \mathrm{~mL}$ round bottom flask. Then the mixture was cooled to $-78{ }^{\circ} \mathrm{C}$ and TMSOTf (310 mg, $1.37 \mathrm{mmol}, 1.0$ equiv.) was added. 4-Fluorophenylmagnesium bromide (1.57 mL, 0.87 M in THF, $1.37 \mathrm{mmol}, 1.0$ equiv.) was added dropwise after $1 \mathrm{~min}$. The mixture was stirred at $-78{ }^{\circ} \mathrm{C}$ for $2 \mathrm{~min}$ and then the temperature was increased to $-30{ }^{\circ} \mathrm{C}$. Methylmagnesium bromide $(0.70 \mathrm{~mL}, 3.0 \mathrm{M}$ in diethyl ether, $2.1 \mathrm{mmol}, 1.5$ equiv.) was then added quickly. The mixture was stirred at $-30{ }^{\circ} \mathrm{C}$ for $10 \mathrm{~min}$. Then the reaction was quenched with sat. aq. tetrasodium EDTA solution and poured into a $250 \mathrm{~mL}$ separating funnel. Ethyl acetate $(80 \mathrm{~mL})$ was then added and the organic phase was separated. The aqueous phase was further extracted with ethyl acetate $(2 \times 40 \mathrm{~mL})$. The combined extracts were dried over anhydrous $\mathrm{Na}_{2} \mathrm{SO}_{4}$, filtered and concentrated under reduced pressure. The crude sulfilimine was dissolved in a mixture of diethyl ether $(15 \mathrm{~mL})$ and petroleum ether $(5 \mathrm{~mL})$ and acidified by a $1 \mathrm{M}$ aq. solution of 4-toluenesulfonic acid (35 $\mathrm{mL})$ and the organic layer was then discarded. The aqueous phase was washed once with a mixture of diethyl ether $(5 \mathrm{~mL})$ and petroleum ether $(15 \mathrm{~mL})$. The aqueous phase was then extracted with $\mathrm{CH}_{2} \mathrm{Cl}_{2}(3 \times 40 \mathrm{~mL})$, dried over anhydrous $\mathrm{Na}_{2} \mathrm{SO}_{4}$, filtered and concentrated in vacuo to afford the 4-toluenesulfonic acid-sulfilimine salt $3 \mathbf{b a}$ (c. 85\% yield). The sulfilimine salt was then dissolved in $\mathrm{CH}_{2} \mathrm{Cl}_{2}(50 \mathrm{~mL})$ and treated with $1 \mathrm{M}$ aq. $\mathrm{NaOH}$ solution $(50 \mathrm{~mL})$. The organic layer was separated and the aqueous phase was extracted with $\mathrm{CH}_{2} \mathrm{Cl}_{2}(2 \times 50 \mathrm{~mL})$. The combined organic extracts were dried over $\mathrm{Na}_{2} \mathrm{SO}_{4}$, filtered and concentrated under reduced pressure to afford sulfilimine $\mathbf{3 b}$ as a light yellow oil (301 mg, 82\%).

(Please see notes on General Procedure C for further guidance.)

Sulfilimine 3b (301 mg, $1.12 \mathrm{mmol}, 1.0$ equiv.) was dissolved in anhydrous MeCN (6.0 mL) in an oven-dried $50 \mathrm{~mL}$ round bottom flask. Then the mixture was heated to $40{ }^{\circ} \mathrm{C}$ before TPAP $(20 \mathrm{mg}$, $0.057 \mathrm{mmol}, 5.0 \mathrm{~mol} \%$ ) was added, then NMO (787 mg, $6.73 \mathrm{mmol}, 6.0$ equiv.) was added. The reaction was stirred at $40{ }^{\circ} \mathrm{C}$ for $24 \mathrm{~h}$ until completion of the reaction (TLC). The reaction was quenched with water and extracted with ethyl acetate $(3 \times 50 \mathrm{~mL})$. The combined extracts were washed with brine, dried over anhydrous $\mathrm{Na}_{2} \mathrm{SO}_{4}$, and concentrated. The crude product was purified by flash column chromatography (petrol/ethyl acetate, 5:1 to 3:1) to afford sulfoximine 5a as a colourless oil (315 mg, 99\%). 
<smiles>NOS(=O)(=O)c1ccc(F)cc1</smiles>

$\mathbf{R}_{f} 0.33$ (petrol/ethyl acetate, 4:1).

${ }^{1} \mathbf{H}$ NMR $\left(400 \mathrm{MHz}, \mathrm{CDCl}_{3}\right): \delta(\mathrm{ppm})=7.97-7.89(\mathrm{~m}, 2 \mathrm{H}), 7.18-7.11(\mathrm{~m}, 2 \mathrm{H}), 2.97(\mathrm{~s}, 3 \mathrm{H}), 1.51(\mathrm{~d}$, $J=14.3 \mathrm{~Hz}, 1 \mathrm{H}), 1.44(\mathrm{~d}, J=14.3 \mathrm{~Hz}, 1 \mathrm{H}), 1.26(\mathrm{~s}, 3 \mathrm{H}), 1.11(\mathrm{~s}, 3 \mathrm{H}), 1.03(\mathrm{~s}, 9 \mathrm{H})$.

${ }^{13} \mathbf{C}$ NMR $\left(100 \mathrm{MHz}, \mathrm{CDCl}_{3}\right): \delta(\mathrm{ppm})=164.9\left(\mathrm{~d},{ }^{1} J_{\mathrm{CF}}=253.0 \mathrm{~Hz}\right), 141.8\left(\mathrm{~d},{ }^{4} J_{\mathrm{CF}}=3.2 \mathrm{~Hz}\right), 130.4$ $\left(\mathrm{d},{ }^{3} J_{\mathrm{CF}}=9.2 \mathrm{~Hz}\right), 116.1\left(\mathrm{~d},{ }^{2} J_{\mathrm{CF}}=22.2 \mathrm{~Hz}\right), 58.8,58.3,48.7,32.9,32.7,32.0,31.9$.

${ }^{19}$ F NMR $\left(376 \mathrm{MHz}, \mathrm{CDCl}_{3}\right): \delta(\mathrm{ppm})=-107.5(\mathrm{tt}, J=8.5,5.2 \mathrm{~Hz})$.

IR (ATR): $\tilde{v}\left(\mathrm{~cm}^{-1}\right)=2956,2903,1589,1491,1363,1226,1150,1090,1077,970,838$

HRMS (ESI ${ }^{+}$) calcd. for $\mathrm{C}_{15} \mathrm{H}_{25} \mathrm{FNOS}^{+}[\mathrm{M}+\mathrm{H}]^{+}: 286.1635$; found: 286.1637 .

(4-Methoxyphenyl)(methyl)((2,4,4-trimethylpentan-2-yl)imino)- $\lambda^{6}$-sulfanone $(5 \mathrm{~b})$<smiles>CCONS(=O)(=O)c1ccc(OC)cc1</smiles>

Sulfilimine prepared according to General Procedure E using $N$-sulfinyl-tert-octylamine 1 (135 mg, 0.770 mmol, 1.05 equiv.), TMSOTf (163 mg, $0.730 \mathrm{mmol}, 1.0$ equiv.), 4-methoxyphenylmagnesium bromide $(1.5 \mathrm{~mL}, 0.49 \mathrm{M}$ in $\mathrm{THF}, 0.74 \mathrm{mmol}, 1.0$ equiv.) and methylmagnesium bromide $(0.37 \mathrm{~mL}, 3.0 \mathrm{M}$ in diethyl ether, $1.1 \mathrm{mmol}, 1.5$ equiv.). Following the general purification method, sulfilimine 3c was generated as light yellow oil with a crude yield of 83\% (170 mg). Sulfoximine prepared using TPAP (11 mg, $0.031 \mathrm{mmol}, 5.0 \mathrm{~mol} \%)$, NMO (0.42 g, $3.6 \mathrm{mmol}, 6.0$ equiv.). Purification by flash column chromatography (petrol/ethyl acetate, $4: 1$ to $2: 1$ ) afforded sulfoximine $\mathbf{5 b}$ as a colourless oil (164 mg, 75\% yield over two steps).

$\mathbf{R}_{f} 0.45$ (petrol/ethyl acetate, 2:1).

${ }^{1} \mathbf{H}$ NMR $\left(400 \mathrm{MHz}, \mathrm{CDCl}_{3}\right): \delta(\mathrm{ppm})=7.85(\mathrm{~d}, J=8.9 \mathrm{~Hz}, 2 \mathrm{H}), 6.96(\mathrm{~d}, J=8.9 \mathrm{~Hz}, 2 \mathrm{H}), 3.85(\mathrm{~s}$, 3H), $2.96(\mathrm{~s}, 3 \mathrm{H}), 1.52(\mathrm{~d}, J=14.3 \mathrm{~Hz}, 1 \mathrm{H}), 1.46(\mathrm{~d}, J=14.3 \mathrm{~Hz}, 1 \mathrm{H}), 1.26(\mathrm{~s}, 3 \mathrm{H}), 1.13$ (s, 3H), 1.04 $(\mathrm{s}, 9 \mathrm{H})$. 
${ }^{13} \mathbf{C}$ NMR $\left(100 \mathrm{MHz}, \mathrm{CDCl}_{3}\right): \delta(\mathrm{ppm})=162.5,137.4,129.8,114.1,58.7,58.4,55.6,48.9,32.9$, $32.7,32.1,31.9$.

IR (ATR): $\tilde{v}\left(\mathrm{~cm}^{-1}\right)=2980,2902,1594,1495,1252,1151,1132,1079,969,833$.

HRMS (ESI ${ }^{+}$) calcd. for $\mathrm{C}_{16} \mathrm{H}_{28} \mathrm{NO}_{2} \mathrm{~S}^{+}[\mathrm{M}+\mathrm{H}]^{+}:$298.1835; found: 298.1836.

\section{Cyclopropyl(4-fluorophenyl $)\left((2,4,4-\right.$ trimethylpentan-2-yl)imino $)-\lambda^{6}$-sulfanone $(5 c)$}<smiles>O=NS(=O)(=O)c1ccc(F)cc1</smiles>

Sulfilimine prepared according to General Procedure E using $N$-sulfinyl-tert-octylamine 1 (231 mg, 1.32 mmol, 1.05 equiv.), TMSOTf (290 mg, $1.31 \mathrm{mmol}, 1.0$ equiv.), cyclopropylmagnesium bromide $(1.55 \mathrm{~mL}, 0.84 \mathrm{M}$ in 2-methyltetrahydrofuran, $1.30 \mathrm{mmol}$, 1.0 equiv.) and 4-fluorophenylmagnesium bromide $(2.00 \mathrm{~mL}, 0.96 \mathrm{M}$ in THF, $1.92 \mathrm{mmol}$, 1.5 equiv.). Following the general purification method, sulfilimine $\mathbf{3 i}$ was generated as light yellow oil with a crude yield of 78\% (300 mg). Sulfoximine prepared using TPAP (18 mg, $0.050 \mathrm{mmol}$, $5.0 \mathrm{~mol} \%$ ), NMO (0.70 g, $6.0 \mathrm{mmol}, 6.0$ equiv.). Purification by flash column chromatography (petrol/ethyl acetate, 5:1 to 3:1) afforded sulfoximine 5c as a white solid (195 mg, 48\% yield over two steps).

mp $77-79{ }^{\circ} \mathrm{C}\left(\mathrm{CH}_{2} \mathrm{Cl}_{2}\right)$

$\mathbf{R}_{f} 0.5$ (petrol/ethyl acetate, $3: 1$ ).

${ }^{1} \mathbf{H}$ NMR $\left(400 \mathrm{MHz}, \mathrm{CDCl}_{3}\right): \delta(\mathrm{ppm})=7.91-7.86(\mathrm{~m}, 2 \mathrm{H}), 7.17-7.11(\mathrm{~m}, 2 \mathrm{H}), 2.39$ (tt, $J=7.8,4.8 \mathrm{~Hz}, 1 \mathrm{H}), 1.51(\mathrm{~d}, J=14.4 \mathrm{~Hz}, 1 \mathrm{H}), 1.46(\mathrm{~d}, J=14.4 \mathrm{~Hz}, 1 \mathrm{H}), 1.43-1.34(\mathrm{~m}, 1 \mathrm{H}), 1.28(\mathrm{~s}$, $3 \mathrm{H}), 1.14(\mathrm{~s}, 3 \mathrm{H}), 1.07-0.94(\mathrm{~m}, 11 \mathrm{H}), 0.80-0.70(\mathrm{~m}, 1 \mathrm{H})$.

${ }^{13} \mathbf{C}$ NMR $\left(100 \mathrm{MHz}, \mathrm{CDCl}_{3}\right): \delta(\mathrm{ppm})=164.7\left(\mathrm{~d},{ }^{1} J_{\mathrm{CF}}=252.7 \mathrm{~Hz}\right), 142.0\left(\mathrm{~d},{ }^{4} J_{\mathrm{CF}}=3.3 \mathrm{~Hz}\right), 130.5$ $\left(\mathrm{d},{ }^{3} J_{\mathrm{CF}}=8.8 \mathrm{~Hz}\right), 115.9\left(\mathrm{~d},{ }^{2} J_{\mathrm{CF}}=22.2 \mathrm{~Hz}\right), 58.5,58.4,36.3,33.2,33.0,32.1,32.0,6.8,5.5$.

${ }^{19}$ F NMR $\left(376 \mathrm{MHz}, \mathrm{CDCl}_{3}\right): \delta(\mathrm{ppm})=-108.1(\mathrm{tt}, J=8.5,5.2 \mathrm{~Hz})$.

IR (ATR): $\tilde{v}\left(\mathrm{~cm}^{-1}\right)=1588,1490,1276,1261,1226,1149,1114,1077,887,836$.

HRMS (ESI ${ }^{+}$) calcd. for $\mathrm{C}_{17} \mathrm{H}_{27} \mathrm{ONFS}^{+}[\mathrm{M}+\mathrm{H}]^{+}$: 312.1792 ; found: 312.1792 . 
<smiles>Cc1ccc(S(=O)(=NO)c2ccc(F)cc2)cc1</smiles>

$\mathbf{R}_{f} 0.65$ (petrol / ethyl acetate $\left.=9: 1\right)$.

${ }^{1} \mathbf{H}$ NMR $\left(400 \mathrm{MHz}, \mathrm{CDCl}_{3}\right): \delta(\mathrm{ppm})=7.97-7.90(\mathrm{~m}, 2 \mathrm{H}), 7.80(\mathrm{~d}, J=8.3 \mathrm{~Hz}, 2 \mathrm{H}), 7.21(\mathrm{~d}$, $J=8.3 \mathrm{~Hz}, 2 \mathrm{H}), 7.09-7.02(\mathrm{~m}, 2 \mathrm{H}), 2.36(\mathrm{~s}, 3 \mathrm{H}), 1.58(\mathrm{~s}, 2 \mathrm{H}), 1.28(\mathrm{~s}, 6 \mathrm{H}), 1.08(\mathrm{~s}, 9 \mathrm{H})$.

${ }^{13} \mathrm{C}$ NMR $\left(100 \mathrm{MHz}, \mathrm{CDCl}_{3}\right): \delta(\mathrm{ppm})=164.5\left(\mathrm{~d},{ }^{1} J_{\mathrm{CF}}=252.7 \mathrm{~Hz}\right), 142.8,142.3,142.1(\mathrm{~d}$, $\left.{ }^{4} J_{\mathrm{CF}}=3.1 \mathrm{~Hz}\right), 130.5\left(\mathrm{~d},{ }^{3} J_{\mathrm{CF}}=9.5 \mathrm{~Hz}\right), 129.6,128.0,115.8\left(\mathrm{~d},{ }^{2} J_{\mathrm{CF}}=22.3 \mathrm{~Hz}\right), 59.0,58.8,33.1,33.0$, $32.11,32.07,21.5$.

${ }^{19}$ F NMR $\left(376 \mathrm{MHz}, \mathrm{CDCl}_{3}\right): \delta(\mathrm{ppm})=-108.3(\mathrm{tt}, J=8.4,5.2 \mathrm{~Hz})$.

IR (ATR): $\tilde{v}\left(\mathrm{~cm}^{-1}\right)=2953,2903,1589,1488,1363,1259,1218,1150,1090,836,813,714,672$.

HRMS (ESI ${ }^{+}$) calcd. for $\mathrm{C}_{21} \mathrm{H}_{29} \mathrm{FNOS}^{+}[\mathrm{M}+\mathrm{H}]^{+}: 362.1948$; found: 362.1946 .

\section{(4-Chlorophenyl)(4-fluorophenyl)((2,4,4-trimethylpentan-2-yl)imino)- $\lambda^{6}$-sulfanone (5e)}<smiles>CCON=S(=O)(c1ccc(F)cc1)c1ccc(Cl)cc1</smiles>

Prepared according to General Procedure D except reaction was carried out at $50{ }^{\circ} \mathrm{C}$ instead of $40{ }^{\circ} \mathrm{C}$, using sulfilimine $3 \mathrm{~g}$ (140 mg, $0.384 \mathrm{mmol}, 1.0$ equiv.), TPAP (6.8 mg, $0.019 \mathrm{mmol}$, $5.0 \mathrm{~mol} \%$ ), NMO (270 mg, $2.31 \mathrm{mmol}, 6.0$ equiv.). Purification by flash column chromatography (petrol/ethyl acetate, 15:1) afforded sulfoximine 5e as a colourless oil (113 mg, 78\%).

$\mathbf{R}_{f} 0.45$ (petrol/ethyl acetate, 7:1).

${ }^{1} \mathbf{H}$ NMR $\left(400 \mathrm{MHz}, \mathrm{CDCl}_{3}\right): \delta(\mathrm{ppm})=7.96-7.90(\mathrm{~m}, 2 \mathrm{H}), 7.85(\mathrm{~d}, J=8.7 \mathrm{~Hz}, 2 \mathrm{H}), 7.38(\mathrm{~d}$, $J=8.7 \mathrm{~Hz}, 2 \mathrm{H}), 7.13-7.05(\mathrm{~m}, 2 \mathrm{H}), 1.58(\mathrm{~s}, 2 \mathrm{H}), 1.28(\mathrm{~s}, 3 \mathrm{H}), 1.27(\mathrm{~s}, 3 \mathrm{H}), 1.07(\mathrm{~s}, 9 \mathrm{H})$.

${ }^{13} \mathbf{C}$ NMR $\left(100 \mathrm{MHz}, \mathrm{CDCl}_{3}\right): \delta(\mathrm{ppm})=164.7\left(\mathrm{~d},{ }^{1} J_{\mathrm{CF}}=253.6 \mathrm{~Hz}\right), 144.3,141.3\left(\mathrm{~d},{ }^{4} J_{\mathrm{CF}}=3.2 \mathrm{~Hz}\right)$, $138.2,130.6\left(\mathrm{~d},{ }^{3} J_{\mathrm{CF}}=9.3 \mathrm{~Hz}\right), 129.4,129.2,116.1\left(\mathrm{~d},{ }^{2} J_{\mathrm{CF}}=22.3 \mathrm{~Hz}\right), 59.2,58.7,33.1,33.0,32.10$, 32.05 .

${ }^{19}$ F NMR $\left(376 \mathrm{MHz}, \mathrm{CDCl}_{3}\right): \delta(\mathrm{ppm})=-107.4(\mathrm{tt}, J=8.2,5.2 \mathrm{~Hz})$. 
IR (ATR): $\tilde{v}\left(\mathrm{~cm}^{-1}\right)=1653,1589,1472,1390,1228,1128,1088,1006,963,813,736$.

HRMS (ESI ${ }^{+}$) calcd. for $\mathrm{C}_{20} \mathrm{H}_{26} \mathrm{~F}^{35} \mathrm{CINOS}^{+}[\mathrm{M}+\mathrm{H}]^{+}$: 382.1402; found: 382.1395 .

\section{(4-Chlorophenyl)(phenyl)((2,4,4-trimethylpentan-2-yl)imino)- $\lambda^{6}$-sulfanone $(5 f)$}<smiles>O=NS(=O)(=O)c1ccccc1</smiles>

Prepared according to General Procedure D except reaction was carried out at $50{ }^{\circ} \mathrm{C}$ instead of $40{ }^{\circ} \mathrm{C}$, using sulfilimine $\mathbf{3 j}$ (210 mg, $0.605 \mathrm{mmol}, 1.0$ equiv.), TPAP (11 mg, $0.031 \mathrm{mmol}$, $5.0 \mathrm{~mol} \%$ ), NMO (428 mg, $3.66 \mathrm{mmol}, 6.0$ equiv.). Purification by flash column chromatography (petrol/ethyl acetate, 10:1 to 6:1) afforded sulfoximine $\mathbf{5 f}$ as a colourless oil (184 mg, 84\%).

$\mathbf{R}_{f} 0.50$ (petrol/ethyl acetate, 15:1).

${ }^{1} \mathbf{H}$ NMR $\left(400 \mathrm{MHz}, \mathrm{CDCl}_{3}\right): \delta(\mathrm{ppm})=7.95-7.91(\mathrm{~m}, 2 \mathrm{H}), 7.88(\mathrm{~d}, J=8.7 \mathrm{~Hz}, 2 \mathrm{H}), 7.46-7.39(\mathrm{~m}$, 3H), $7.37(\mathrm{~d}, J=8.7 \mathrm{~Hz}, 2 \mathrm{H}), 1.59(\mathrm{~s}, 2 \mathrm{H}), 1.283(\mathrm{~s}, 3 \mathrm{H}), 1.278(\mathrm{~s}, 3 \mathrm{H}), 1.08(\mathrm{~s}, 9 \mathrm{H})$.

${ }^{13} \mathbf{C}$ NMR $\left(100 \mathrm{MHz}, \mathrm{CDCl}_{3}\right): \delta(\mathrm{ppm})=145.3,144.4,138.0,131.7,129.5,129.0,128.9,127.9$, 59.1, 58.6, 33.1, 33.0, 32.1, 32.0.

IR (ATR): $\tilde{v}\left(\mathrm{~cm}^{-1}\right)=1473,1276,1261,1213,1153,1087,1066,1013,827,749,701$.

HRMS $\left(\mathrm{ESI}^{+}\right)$calcd. for $\mathrm{C}_{20} \mathrm{H}_{27} \mathrm{O}^{35} \mathrm{CINS}^{+}[\mathrm{M}+\mathrm{H}]^{+}$: 364.1496; found: 364.1500 .

\section{(4-Methoxyphenyl $)(p$-tolyl $)\left((2,4,4\right.$-trimethylpentan-2-yl)imino $)-\lambda^{6}$-sulfanone $(5 \mathrm{~g})$}<smiles>CCON=S(=O)(c1ccc(C)cc1)c1ccc(OC)cc1</smiles>

Prepared according to General Procedure D using sulfilimine 3r (155 mg, 0.434 mmol, 1.0 equiv.), TPAP (8.1 mg, $0.023 \mathrm{mmol}, 5.0 \mathrm{~mol} \%$ ), NMO (310 mg, $2.65 \mathrm{mmol}, 6.0$ equiv.). Purification by flash column chromatography (petrol/ethyl acetate, 7:1) afforded sulfoximine $\mathbf{5 g}$ as a colourless oil (142 mg, 88\%).

$\mathbf{R}_{f} 0.40$ (petrol/ethyl acetate, 5:1). 
${ }^{1} \mathbf{H}$ NMR $\left(400 \mathrm{MHz}, \mathrm{CDCl}_{3}\right): \delta(\mathrm{ppm})=7.86(\mathrm{~d}, J=8.9 \mathrm{~Hz}, 2 \mathrm{H}), 7.80(\mathrm{~d}, J=8.3 \mathrm{~Hz}, 2 \mathrm{H}), 7.18(\mathrm{~d}$, $J=8.0 \mathrm{~Hz}, 2 \mathrm{H}), 6.87(\mathrm{~d}, J=8.9 \mathrm{~Hz}, 2 \mathrm{H}), 3.78(\mathrm{~s}, 3 \mathrm{H}), 2.33(\mathrm{~s}, 3 \mathrm{H}), 1.58(\mathrm{~s}, 2 \mathrm{H}), 1.28(\mathrm{~s}, 3 \mathrm{H}), 1.27$ (s, 3H), 1.09 (s, 9H).

${ }^{13} \mathrm{C}$ NMR (100 MHz, $\left.\left(\mathrm{CD}_{3}\right)_{2} \mathrm{CO}\right): \delta(\mathrm{ppm})=163.3,145.0,142.9,138.9,130.8,130.4,128.7,115.0$, 59.6, 59.1, 56.2, 33.8, 33.7, 32.8, 32.7, 21.6.

IR (ATR): $\tilde{v}\left(\mathrm{~cm}^{-1}\right)=1594,1492,1387,1251,1151,1091,1027,951,801,716,673$.

HRMS (ESI ${ }^{+}$) calcd. for $\mathrm{C}_{22} \mathrm{H}_{32} \mathrm{NO}_{2} \mathrm{~S}^{+}[\mathrm{M}+\mathrm{H}]^{+}:$374.2148; found: 374.2146 .

(4-Methoxyphenyl)(m-tolyl)((2,4,4-trimethylpentan-2-yl)imino $)-\lambda^{6}$-sulfanone $(5 \mathrm{~h})$<smiles>CON=S(=O)(c1ccc(OC)cc1)c1cccc(C)c1</smiles>

Prepared according to General Procedure D except reaction was carried out at $50{ }^{\circ} \mathrm{C}$ instead of $40{ }^{\circ} \mathrm{C}$, using sulfilimine $3 \mathrm{~s}$ (135 mg, $0.378 \mathrm{mmol}, 1.0$ equiv.), TPAP (6.9 mg, $0.019 \mathrm{mmol}$, $5.0 \mathrm{~mol} \%$ ), NMO (263 mg, $2.25 \mathrm{mmol}, 6.0$ equiv.). Purification by flash column chromatography (petrol/ethyl acetate, 12:1) afforded sulfoximine $\mathbf{5 h}$ as a colorless oil (128 $\mathrm{mg}, 91 \%)$.

$\mathbf{R}_{f} 0.46$ (petrol/ethyl acetate, 9:1).

${ }^{1} \mathbf{H}$ NMR $\left(400 \mathrm{MHz}, \mathrm{CDCl}_{3}\right): \delta(\mathrm{ppm})=7.87(\mathrm{~d}, J=8.9 \mathrm{~Hz}, 2 \mathrm{H}), 7.76-7.69(\mathrm{~m}, 2 \mathrm{H}), 7.29-7.24(\mathrm{~m}$, 1H), 7.22-7.18 (m, 1H), $6.88(\mathrm{~d}, J=8.9 \mathrm{~Hz}, 2 \mathrm{H}), 3.78(\mathrm{~s}, 3 \mathrm{H}), 2.35(\mathrm{~s}, 3 \mathrm{H}), 1.59(\mathrm{~s}, 2 \mathrm{H}), 1.282(\mathrm{~s}$, $3 \mathrm{H}), 1.277(\mathrm{~s}, 3 \mathrm{H}), 1.09(\mathrm{~s}, 9 \mathrm{H})$.

${ }^{13}$ C NMR (100 MHz, $\left.\left(\mathrm{CD}_{3}\right)_{2} \mathrm{CO}\right): \delta(\mathrm{ppm})=163.4,147.7,139.8,138.7,133.0,130.9,129.7,128.9$, $125.9,115.0,59.6,59.1,56.2,33.8,33.7,32.8,32.7,21.6$.

IR (ATR): $\tilde{v}\left(\mathrm{~cm}^{-1}\right)=1594,1493,1386,1252,1151,1090,955,832,694$.

HRMS (ESI ${ }^{+}$) calcd. for $\mathrm{C}_{22} \mathrm{H}_{32} \mathrm{NO}_{2} \mathrm{~S}^{+}[\mathrm{M}+\mathrm{H}]^{+}:$374.2148; found: 374.2140.

(4-Methoxyphenyl)(o-tolyl $)\left((2,4,4-\right.$ trimethylpentan-2-yl)imino $)-\lambda^{6}$-sulfanone (5i)

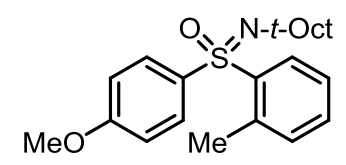


Prepared according to General Procedure D using sulfilimine 3t (60 mg, 0.17 mmol, 1.0 equiv.), TPAP (9.2 mg, $0.026 \mathrm{mmol}, 15 \mathrm{~mol} \%$ ), NMO (117 mg, $1.00 \mathrm{mmol}, 6.0$ equiv.). Purification by flash column chromatography (petrol/ethyl acetate, 12:1) afforded sulfoximine $\mathbf{5 i}$ as a colourless oil (45 mg, 72\%).

$\mathbf{R}_{f} 0.50$ (petrol/ethyl acetate, 9:1).

${ }^{1} \mathbf{H}$ NMR $\left(400 \mathrm{MHz}, \mathrm{CDCl}_{3}\right): \delta(\mathrm{ppm})=8.36(\mathrm{dd}, J=7.6,1.9 \mathrm{~Hz}, 1 \mathrm{H}), 7.80(\mathrm{~d}, J=9.0 \mathrm{~Hz}, 2 \mathrm{H})$, 7.37-7.28 (m, 2H), 7.12-7.08 (m, 1H), $6.88(\mathrm{~d}, J=9.0 \mathrm{~Hz}, 2 \mathrm{H}), 3.81(\mathrm{~s}, 3 \mathrm{H}), 2.33(\mathrm{~s}, 3 \mathrm{H}), 1.59$ (s, 2H), $1.27(\mathrm{~s}, 3 \mathrm{H}), 1.21(\mathrm{~s}, 3 \mathrm{H}), 1.08(\mathrm{~s}, 9 \mathrm{H})$.

${ }^{13} \mathrm{C}$ NMR $\left(100 \mathrm{MHz}, \mathrm{CDCl}_{3}\right): \delta(\mathrm{ppm})=162.0,143.9,137.5,136.6,132.6,131.7,130.2,130.1$, $126.1,113.7,59.0,58.7,55.6,32.7,32.5,32.14,32.07,20.2$.

IR (ATR): $\tilde{v}\left(\mathrm{~cm}^{-1}\right)=1594,1494,1251,1151,1083,833,803,759,717$.

HRMS $\left(\mathrm{ESI}^{+}\right.$) calcd. for $\mathrm{C}_{22} \mathrm{H}_{32} \mathrm{NO}_{2} \mathrm{~S}^{+}[\mathrm{M}+\mathrm{H}]^{+}: 374.2148$; found:374.2138.

(4-Methoxyphenyl)(naphthalen-2-yl)((2,4,4-trimethylpentan-2-yl)imino)- $\lambda^{6}$-sulfanone $(5 j)$<smiles>COc1ccc(S(=O)(=O)NOc2ccc3ccccc3c2)cc1</smiles>

Prepared according to General Procedure D using sulfilimine 3u (153 mg, 0.389 mmol, 1.0 equiv.), TPAP (6.9 mg, 0.019 mmol, $5.0 \mathrm{~mol} \%$ ), NMO (277 mg, $2.37 \mathrm{mmol}, 6.0$ equiv.). Purification by flash column chromatography (petrol/ethyl acetate, 9:1) afforded sulfoximine $\mathbf{5 j}$ as a white solid (146 mg, 92\%).

mp $106-108{ }^{\circ} \mathrm{C}\left(\mathrm{CH}_{2} \mathrm{Cl}_{2}\right)$

$\mathbf{R}_{f} 0.45$ (petrol/ethyl acetate, 5:1).

${ }^{1} \mathbf{H}$ NMR $\left(400 \mathrm{MHz}, \mathrm{CDCl}_{3}\right): \delta(\mathrm{ppm})=8.61-8.55(\mathrm{~m}, 1 \mathrm{H}), 7.98-7.91(\mathrm{~m}, 3 \mathrm{H}), 7.87(\mathrm{dd}$, $J=8.7,1.8 \mathrm{~Hz}, 1 \mathrm{H}), 7.85-7.79(\mathrm{~m}, 2 \mathrm{H}), 7.58-7.51(\mathrm{~m}, 2 \mathrm{H}), 6.89(\mathrm{~d}, J=9.0 \mathrm{~Hz}, 2 \mathrm{H}), 3.78(\mathrm{~s}, 3 \mathrm{H})$, $1.66(\mathrm{~d}, J=14.4 \mathrm{~Hz}, 1 \mathrm{H}), 1.62(\mathrm{~d}, J=14.4 \mathrm{~Hz}, 1 \mathrm{H}), 1.35$ (s, 3H), 1.30 (s, 3H), $1.14(\mathrm{~s}, 9 \mathrm{H})$.

${ }^{13} \mathrm{C}$ NMR $\left(100 \mathrm{MHz}, \mathrm{CDCl}_{3}\right): \delta(\mathrm{ppm})=162.2,143.5,137.1,134.3,132.6,130.1,129.3,128.8$, $128.4,128.2,127.8,127.1,124.0,114.0,58.9,58.8,55.6,33.3,33.0,32.12,32.10$. 
IR (ATR): $\tilde{v}\left(\mathrm{~cm}^{-1}\right)=1593,1493,1386,1254,1152,1083,955,832,748$.

HRMS (ESI ${ }^{+}$) calcd. for $\mathrm{C}_{25} \mathrm{H}_{32} \mathrm{NO}_{2} \mathrm{~S}^{+}[\mathrm{M}+\mathrm{H}]^{+}:$410.2148; found: 410.2141 .

\section{(4-Chlorophenyl)(6-methoxypyridin-3-yl)((2,4,4-trimethylpentan-2-yl)imino)- $\lambda^{6}$-sulfanone}

(5k)<smiles>COc1ccc(S(=O)(=O)c2ccc(Cl)cc2)cn1</smiles>

Prepared according to General Procedure D using sulfilimine 3k (96.6 mg, $0.256 \mathrm{mmol}$, 1.0 equiv.), TPAP (4.5 mg, $0.013 \mathrm{mmol}, 5.0 \mathrm{~mol} \%$ ), NMO (180 mg, $1.54 \mathrm{mmol}, 6.0$ equiv.). Purification by flash column chromatography (petrol/ethyl acetate, 10:1) afforded sulfoximine 5k as a colourless oil $(88 \mathrm{mg}, 88 \%)$.

$\mathbf{R}_{f} 0.50$ (petrol/ethyl acetate, 5:1).

${ }^{1} \mathbf{H}$ NMR $\left(400 \mathrm{MHz}, \mathrm{CDCl}_{3}\right): \delta(\mathrm{ppm})=8.72(\mathrm{dd}, J=2.5,0.7 \mathrm{~Hz}, 1 \mathrm{H}), 7.95(\mathrm{dd}, J=8.8,2.5 \mathrm{~Hz}, 1 \mathrm{H})$, $7.85(\mathrm{~d}, J=8.7 \mathrm{~Hz}, 2 \mathrm{H}), 7.38(\mathrm{~d}, J=8.7 \mathrm{~Hz}, 2 \mathrm{H}), 6.72(\mathrm{dd}, J=8.8,0.7 \mathrm{~Hz}, 1 \mathrm{H}), 3.94(\mathrm{~s}, 3 \mathrm{H}), 1.57$ (s, 2H), $1.28(\mathrm{~s}, 3 \mathrm{H}), 1.26(\mathrm{~s}, 3 \mathrm{H}), 1.06(\mathrm{~s}, 9 \mathrm{H})$.

${ }^{13} \mathrm{C}$ NMR $\left(100 \mathrm{MHz}, \mathrm{CDCl}_{3}\right): \delta(\mathrm{ppm})=165.9,148.2,144.4,138.2,138.1,134.4,129.3,129.2$, $111.2,59.2,58.6,54.3,33.2,33.1,32.1,32.0$.

IR (ATR): $\tilde{v}\left(\mathrm{~cm}^{-1}\right)=1587,1476,1366,1276,1261,1212,1154,1118,1087,1013,823$.

HRMS (ESI ${ }^{+}$) calcd. for $\mathrm{C}_{20} \mathrm{H}_{28}{ }^{35} \mathrm{ClO}_{2} \mathrm{~N}_{2} \mathrm{~S}^{+}[\mathrm{M}+\mathrm{H}]^{+}$: 395.1555 ; found: 395.1553 .

\section{(4-Chlorophenyl)(thiophen-2-yl)((2,4,4-trimethylpentan-2-yl)imino)- $\lambda^{6}$-sulfanone (5l)}<smiles>O=N[SH](=O)(c1ccc(Cl)cc1)c1cccs1</smiles>

Prepared according to General Procedure D except reaction was carried out at $50{ }^{\circ} \mathrm{C}$ instead of $40{ }^{\circ} \mathrm{C}$, using sulfilimine 31 (223 mg, $0.632 \mathrm{mmol}, 1.0$ equiv.), TPAP (12 mg, $0.034 \mathrm{mmol}$, $5.0 \mathrm{~mol} \%$ ), NMO (447 mg, $3.82 \mathrm{mmol}, 6.0$ equiv.). Purification by flash column chromatography (petrol/ethyl acetate, 11:1 to 6:1) afforded sulfoximine $\mathbf{5 l}$ as a colourless oil (201 $\mathrm{mg}, 87 \%$ ).

$\mathbf{R}_{f} 0.50$ (petrol/ethyl acetate, 15:1). 
${ }^{1} \mathbf{H}$ NMR $\left(400 \mathrm{MHz}, \mathrm{CDCl}_{3}\right): \delta(\mathrm{ppm})=7.95(\mathrm{~d}, J=8.7 \mathrm{~Hz}, 2 \mathrm{H}), 7.50(\mathrm{dd}, J=5.0,1.3 \mathrm{~Hz}, 1 \mathrm{H}), 7.43$ $(\mathrm{dd}, J=3.7,1.3 \mathrm{~Hz}, 1 \mathrm{H}), 7.39$ (d, $J=8.7 \mathrm{~Hz}, 2 \mathrm{H}), 6.98(\mathrm{dd}, J=5.0,3.7 \mathrm{~Hz}, 1 \mathrm{H}), 1.58(\mathrm{~s}, 2 \mathrm{H}), 1.334$ (s, 3H), 1.329 (s, 3H), $1.08(\mathrm{~s}, 9 \mathrm{H})$.

${ }^{13} \mathrm{C}$ NMR $\left(100 \mathrm{MHz}, \mathrm{CDCl}_{3}\right): \delta(\mathrm{ppm})=148.5,144.6,138.1,132.5,131.9,129.1,129.0,127.8,59.4$, $58.5,33.1,32.7,32.1,32.0$.

IR (ATR): $\tilde{v}\left(\mathrm{~cm}^{-1}\right)=1473,1276,1261,1212,1154,1088,1012,997,826,750$.

HRMS (ESI ${ }^{+}$) calcd. for $\mathrm{C}_{18} \mathrm{H}_{25} \mathrm{O}^{35} \mathrm{ClNS}_{2}{ }^{+}[\mathrm{M}+\mathrm{H}]^{+}: 370.1061$; found: 370.1059 .

\section{Butyl(2-methylallyl $)\left((2,4,4-\right.$ trimethylpentan-2-yl)imino $)-\lambda^{6}$-sulfanone (5m)}<smiles>C=C(C)CS(=O)(CCCC)=NOCC</smiles>

Sulfilimine prepared according to General Procedure E using $N$-sulfinyl-tert-octylamine 1 (512 mg, $2.92 \mathrm{mmol}, 1.05$ equiv.), TMSOTf (605 mg, $2.72 \mathrm{mmol}, 1.0$ equiv.), $n$-butylmagnesium chloride (1.39 mL, 1.96 M in THF, $2.72 \mathrm{mmol}, 1.0$ equiv.) and 2-methyl-1-propenylmagnesium bromide (8.00 mL, 0.49 M in THF, $3.92 \mathrm{mmol}$, 1.5 equiv.). Following the general purification method, sulfilimine 3o was generated as light yellow oil with a crude yield of $81 \%(600 \mathrm{mg})$. Sulfoximine prepared using TPAP (39 mg, $0.11 \mathrm{mmol}, 5.0 \mathrm{~mol} \%$ ), NMO (1.54 g, $13.2 \mathrm{mmol}$, 6.0 equiv.). Purification by flash column chromatography (petrol/ethyl acetate, $3: 1$ to 1:1) afforded sulfoximine $\mathbf{5 m}$ as a light yellow oil (187 $\mathrm{mg}, 24 \%$ yield over two steps).

$\mathbf{R}_{f} 0.61$ (petrol/ethyl acetate, 1:1).

${ }^{1} \mathbf{H}$ NMR $\left(400 \mathrm{MHz}, \mathrm{CDCl}_{3}\right): \delta(\mathrm{ppm})=4.93(\mathrm{~s}, 1 \mathrm{H}), 4.84(\mathrm{~s}, 1 \mathrm{H}), 3.98(\mathrm{~d}, J=19.6 \mathrm{~Hz}, 1 \mathrm{H}), 3.03(\mathrm{~d}$, $J=19.6 \mathrm{~Hz}, 1 \mathrm{H}), 2.55-2.38(\mathrm{~m}, 2 \mathrm{H}), 1.76(\mathrm{~d}, J=14.7 \mathrm{~Hz}, 1 \mathrm{H}), 1.70(\mathrm{~s}, 3 \mathrm{H}), 1.68(\mathrm{~d}, J=14.7 \mathrm{~Hz}, 1 \mathrm{H})$, 1.62-1.47 (m, 2H), 1.43-1.31 (m, 5H), $1.22(\mathrm{~s}, 3 \mathrm{H}), 0.95(\mathrm{~s}, 9 \mathrm{H}), 0.87(\mathrm{t}, J=7.3 \mathrm{~Hz}, 3 \mathrm{H})$.

${ }^{13} \mathrm{C}$ NMR $\left(100 \mathrm{MHz}, \mathrm{CDCl}_{3}\right): \delta(\mathrm{ppm})=145.6,110.7,63.6,54.5,53.1,42.0,31.9,31.4,29.3,27.5$, $25.9,22.0,20.7,13.8$.

IR (ATR): $\tilde{v}\left(\mathrm{~cm}^{-1}\right)=1469,1368,1276,1261,1138,1071,1045,895,839,763$.

HRMS $\left(\mathrm{ESI}^{+}\right)$calcd. for $\mathrm{C}_{16} \mathrm{H}_{34} \mathrm{NOS}^{+}[\mathrm{M}+\mathrm{H}]^{+}:$288.2356; found: 288.2354. 
Cyclopropyl(2-methylallyl)((2,4,4-trimethylpentan-2-yl)imino $)-\lambda^{6}$-sulfanone (5n)<smiles>C=C(C)CS(=O)(=O)C1CC1</smiles>

Sulfilimine prepared according to General Procedure E using $N$-sulfinyl-tert-octylamine 1 (340 mg, 1.94 mmol, 1.05 equiv.), TMSOTf (416 mg, 1.87 mmol, 1.0 equiv.), cyclopropylmagnesium bromide $(2.23 \mathrm{~mL}, 0.84 \mathrm{M}$ in 2-methyltetrahydrofuran, $1.87 \mathrm{mmol}$, 1.0 equiv.) and 2-methyl-1-propenylmagnesium bromide $(5.60 \mathrm{~mL}, 0.49 \mathrm{M}$ in THF, $2.74 \mathrm{mmol}$, 1.5 equiv.). Following the general purification method, sulfilimine $\mathbf{3 q}$ was generated as light yellow oil with a crude yield of $81 \%$ (387 mg). Sulfoximine prepared using TPAP (27 mg, $0.076 \mathrm{mmol}$, $5.0 \mathrm{~mol} \%$ ), NMO (1.07 g, $9.15 \mathrm{mmol}, 6.0$ equiv.). Purification by flash column chromatography (petrol/ethyl acetate, 4:1 to 2:1) afforded sulfoximine 5n as a colourless oil (236 $\mathrm{mg}, 47 \%$ yield over two steps).

$\mathbf{R}_{f} 0.65$ (petrol/ethyl acetate, 3:1).

${ }^{1} \mathbf{H}$ NMR $\left(400 \mathrm{MHz}, \mathrm{CDCl}_{3}\right): \delta(\mathrm{ppm})=4.96(\mathrm{~s}, 1 \mathrm{H}), 4.78(\mathrm{~s}, 1 \mathrm{H}), 3.88(\mathrm{~d}, J=19.8 \mathrm{~Hz}, 1 \mathrm{H}), 3.05(\mathrm{~d}$, $J=19.8 \mathrm{~Hz}, 1 \mathrm{H}), 1.88(\mathrm{tt}, J=8.0,5.0 \mathrm{~Hz}, 1 \mathrm{H}), 1.79(\mathrm{~d}, J=14.7 \mathrm{~Hz}, 1 \mathrm{H}), 1.65(\mathrm{~s}, 3 \mathrm{H}), 1.64(\mathrm{~d}$, $J=14.7 \mathrm{~Hz}, 1 \mathrm{H}), 1.37(\mathrm{~s}, 3 \mathrm{H}), 1.21(\mathrm{~s}, 3 \mathrm{H}), 1.18-1.11(\mathrm{~m}, 1 \mathrm{H}), 0.93(\mathrm{~s}, 9 \mathrm{H}), 0.65-0.54(\mathrm{~m}, 2 \mathrm{H})$, 0.52-0.44 (m, 1H).

${ }^{13} \mathrm{C}$ NMR $\left(100 \mathrm{MHz}, \mathrm{CDCl}_{3}\right): \delta(\mathrm{ppm})=144.6,110.2,63.4,53.3,42.9,31.9,31.4,30.1,29.2,27.6$, $20.6,2.7,-0.1$.

IR (ATR): $\tilde{v}\left(\mathrm{~cm}^{-1}\right)=1472,1368,1276,1261,1139,1071,1028,896,840,818,750$.

HRMS $\left(\mathrm{ESI}^{+}\right)$calcd. for $\mathrm{C}_{15} \mathrm{H}_{30} \mathrm{NOS}^{+}[\mathrm{M}+\mathrm{H}]^{+}:$272.2043; found: 272.2043 .

\section{(4-Chlorophenyl)(4-(5-(p-tolyl)-3-(trifluoromethyl)-1H-pyrazol-1-yl)phenyl)((2,4,4-} trimethylpentan-2-yl)imino)- $\lambda^{6}$-sulfanone (5o)<smiles>Cc1ccc(-c2cc(C(F)(F)F)nn2-c2ccc(S(=O)(=O)N=O)cc2)cc1</smiles> 
Prepared according to General Procedure D using sulfilimine 3n (108 mg, 0.189 mmol, 1.0 equiv.), TPAP (3.4 mg, $0.010 \mathrm{mmol}, 5.0 \mathrm{~mol} \%$ ), NMO (137 mg, $1.17 \mathrm{mmol}, 6.0$ equiv.). Purification by flash column chromatography (petrol/ethyl acetate, 10:1) afforded sulfoximine 5o as a colourless oil (83 mg, 75\%).

$\mathbf{R}_{f} 0.5$ (petrol/ethyl acetate, 9:1).

${ }^{1}$ H NMR $\left(400 \mathrm{MHz}, \mathrm{CDCl}_{3}\right): \delta(\mathrm{ppm})=7.90(\mathrm{~d}, J=8.7 \mathrm{~Hz}, 2 \mathrm{H}), 7.84(\mathrm{~d}, J=8.7 \mathrm{~Hz}, 2 \mathrm{H}), 7.40(\mathrm{~d}$, $J=6.1 \mathrm{~Hz}, 2 \mathrm{H}), 7.37(\mathrm{~d}, J=6.1 \mathrm{~Hz}, 2 \mathrm{H}), 7.13(\mathrm{~d}, J=7.8 \mathrm{~Hz}, 2 \mathrm{H}), 7.07$ (d, $J=7.8 \mathrm{~Hz}, 2 \mathrm{H}), 6.71(\mathrm{~s}$, 1H), $2.37(\mathrm{~s}, 3 \mathrm{H}), 1.58(\mathrm{~s}, 2 \mathrm{H}), 1.28(\mathrm{~s}, 3 \mathrm{H}), 1.27(\mathrm{~s}, 3 \mathrm{H}), 1.06(\mathrm{~s}, 9 \mathrm{H})$.

${ }^{13} \mathrm{C}$ NMR $\left(100 \mathrm{MHz}, \mathrm{CDCl}_{3}\right): \delta(\mathrm{ppm})=145.2,144.9,144.0\left(\mathrm{q},{ }^{2} J_{\mathrm{CF}}=38.3 \mathrm{~Hz}\right), 143.8,141.8,139.8$, $138.4,129.7,129.6,129.2,129.0,128.8,125.9,125.5,121.2\left(\mathrm{q},{ }^{1} J_{\mathrm{CF}}=269.2 \mathrm{~Hz}\right), 106.2,59.3,58.6$, $33.1,33.0,32.1,32.0,21.5$.

${ }^{19}$ F NMR $\left(376 \mathrm{MHz}, \mathrm{CDCl}_{3}\right): \delta(\mathrm{ppm})=-62.4$

IR (ATR): $\tilde{v}\left(\mathrm{~cm}^{-1}\right)=1472,1276,1261,1235,1214,1134,1088,975,909,750$.

HRMS $\left(\mathrm{ESI}^{+}\right.$) calcd. for $\mathrm{C}_{31} \mathrm{H}_{34} \mathrm{O}^{35} \mathrm{ClN}_{3} \mathrm{~F}_{3} \mathrm{~S}^{+}[\mathrm{M}+\mathrm{H}]^{+}:$: 588.2058; found: 588.2055.

\subsubsection{Deprotection of Sulfondiimine}

$N$-((4-Fluorophenyl)(imino)(methyl)- $\lambda^{6}$-sulfaneylidene)-4-nitrobenzenesulfonamide (6)<smiles>NS(=O)(=O)c1ccc(F)cc1</smiles>

Sulfondiimine 4b (1.00 g, 2.14 mmol, 1.0 equiv.) was mixed with TFA (21 mL) and stirred at room temperature for $14 \mathrm{~h}$. The mixture was concentrated in vacuo then diluted with $\mathrm{CH}_{2} \mathrm{Cl}_{2}$ and basified to $\mathrm{pH}$ 10-11 using $1 \mathrm{M}$ aq. $\mathrm{NaOH}$ solution. The product was extracted with $\mathrm{CH}_{2} \mathrm{Cl}_{2}(3 \times 50 \mathrm{~mL})$. The combined extracts were washed with brine, dried over anhydrous $\mathrm{Na}_{2} \mathrm{SO}_{4}$, and concentrated in vасио. The crude product was purified by flash column chromatography (petrol/ethyl acetate, 1:1 to 1:3) to afford sulfondiimine $\mathbf{6}$ as a white solid (737 $\mathrm{mg}, 97 \%$ ).

mp $173-175^{\circ} \mathrm{C}\left(\mathrm{CH}_{2} \mathrm{Cl}_{2}\right)$

$\mathbf{R}_{f} 0.37$ (petrol/ethyl acetate, 1:3). 
${ }^{1} \mathbf{H}$ NMR $\left(400 \mathrm{MHz}, \mathrm{CDCl}_{3}\right): \delta(\mathrm{ppm})=8.29(\mathrm{~d}, J=8.9 \mathrm{~Hz}, 2 \mathrm{H}), 8.13(\mathrm{~d}, J=8.9 \mathrm{~Hz}, 2 \mathrm{H}), 8.15-8.08$ (m, 2H), 7.29-7.24 (m, 2H), 3.43 (s, 3H), 2.71 (br. s, 1H).

${ }^{13}$ C NMR (100 MHz, $\left.\left(\mathrm{CD}_{3}\right)_{2} \mathrm{CO}\right): \delta(\mathrm{ppm})=166.7\left(\mathrm{~d},{ }^{1} J_{\mathrm{CF}}=253.7 \mathrm{~Hz}\right), 151.5,150.5,138.5(\mathrm{~d}$, $\left.{ }^{4} J_{\mathrm{CF}}=3.2 \mathrm{~Hz}\right), 131.6\left(\mathrm{~d},{ }^{3} J_{\mathrm{CF}}=9.6 \mathrm{~Hz}\right), 129.0,124.8,117.4\left(\mathrm{~d},{ }^{2} J_{\mathrm{CF}}=23.1 \mathrm{~Hz}\right), 48.5$.

${ }^{19}$ F NMR $\left(376 \mathrm{MHz}, \mathrm{CDCl}_{3}\right): \delta(\mathrm{ppm})=-102.4(\mathrm{tt}, J=8.0,4.9 \mathrm{~Hz})$.

IR (ATR): $\tilde{v}\left(\mathrm{~cm}^{-1}\right)=2978,1528,1352,1296,1238,1152,1086,1035,1006,968$.

HRMS $\left(\mathrm{ESI}^{+}\right)$calcd. for $\mathrm{C}_{13} \mathrm{H}_{13} \mathrm{FN}_{3} \mathrm{O}_{4} \mathrm{~S}_{2}^{+}[\mathrm{M}+\mathrm{H}]^{+}:$358.0326; found: 358.0323 .

\section{1-(4-Fluorophenyl)-1-methyl- $N$-(2,4,4-trimethylpentan-2-yl)- $\lambda^{6}$-sulfanediimine (7)}

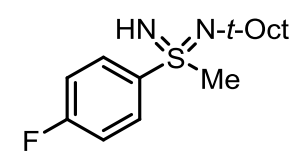

Under an atmosphere of nitrogen, sulfondiimines $\mathbf{4 b}$ (1.50 g, $3.20 \mathrm{mmol}, 1.0$ equiv.) was dissolved in anhydrous MeCN (10 ml). 1-Dodecanethiol $(3.80 \mathrm{ml}, 15.8 \mathrm{mmol}, 5.0$ equiv.) was added at room temperature followed by DBU $(2.30 \mathrm{~mL}, 15.4 \mathrm{mmol}, 4.75$ equiv.). The solution became yellow and was stirred for $20 \mathrm{~min}$ under nitrogen until completion of the reaction (TLC). The reaction mixture was then concentrated in vacuo to afford light yellow oil. The crude product was purified by flash column chromatography (petrol/ethyl acetate, 1:1 to $0: 1$ ) to afford corresponding sulfondiimine 7 as a colourless oil (806 mg, 89\%).

$\mathbf{R}_{f} 0.44$ (ethyl acetate).

${ }^{1} \mathbf{H}$ NMR $\left(400 \mathrm{MHz}, \mathrm{CDCl}_{3}\right): \delta(\mathrm{ppm})=8.14-8.08(\mathrm{~m}, 2 \mathrm{H}), 7.18-7.08$ (m, 2H), 3.05 (s, 3H), 1.97 (br. s, $1 \mathrm{H}), 1.56(\mathrm{~d}, J=14.4 \mathrm{~Hz}, 1 \mathrm{H}), 1.52(\mathrm{~d}, J=14.4 \mathrm{~Hz}, 1 \mathrm{H}), 1.41(\mathrm{~s}, 3 \mathrm{H}), 1.30(\mathrm{~s}, 3 \mathrm{H}), 1.03$ (s, 9H).

${ }^{13} \mathbf{C}$ NMR $\left(100 \mathrm{MHz}, \mathrm{CDCl}_{3}\right): \delta(\mathrm{ppm})=164.6\left(\mathrm{~d},{ }^{1} J_{\mathrm{CF}}=252.7 \mathrm{~Hz}\right), 143.0\left(\mathrm{~d},{ }^{4} J_{\mathrm{CF}}=3.1 \mathrm{~Hz}\right), 129.8$ $\left(\mathrm{d},{ }^{3} J_{\mathrm{CF}}=9.1 \mathrm{~Hz}\right), 115.7\left(\mathrm{~d},{ }^{2} J_{\mathrm{CF}}=22.4 \mathrm{~Hz}\right), 58.72,58.70,51.8,33.3,32.3,32.08,32.05$.

${ }^{19}$ F NMR $\left(376 \mathrm{MHz}, \mathrm{CDCl}_{3}\right): \delta(\mathrm{ppm})=-108.7(\mathrm{tt}, J=8.3,5.2 \mathrm{~Hz})$.

IR (ATR): $\tilde{v}\left(\mathrm{~cm}^{-1}\right)=2954,1589,1488,1219,1188,1152,1087,1001,939$.

HRMS $\left(\mathrm{ESI}^{+}\right.$) calcd. for $\mathrm{C}_{15} \mathrm{H}_{26} \mathrm{FN}_{2} \mathrm{~S}^{+}[\mathrm{M}+\mathrm{H}]^{+}:$285.1795; found: 285.1795 . 


\section{(4-Fluorophenyl)(methyl)- $\lambda^{6}$-sulfanediimine (8)}<smiles>CS(=N)(=N)c1ccc(F)cc1</smiles>

Under an atmosphere of nitrogen, sulfondiimines 6 (602 mg, $1.69 \mathrm{mmol}, 1.0$ equiv.) was dissolved in anhydrous $\mathrm{MeCN}(5.0 \mathrm{ml})$. 1-Dodecanethiol $(2.00 \mathrm{~mL}, 8.35 \mathrm{mmol}, 5.0$ equiv.) was added at room temperature followed by DBU (1.20 mL, $8.02 \mathrm{mmol}, 4.75$ equiv.). The solution became yellow and was stirred for 20 min under nitrogen until completion of the reaction (TLC). The reaction mixture was concentrated in vacuo to afford light yellow oil. The crude product was purified by flash column chromatography $\left(\mathrm{CH}_{2} \mathrm{Cl}_{2} / \mathrm{MeOH}, 9: 1\right)$ to afford sulfondiimine $\mathbf{8}$ as a white solid (285 mg, 98\%).

mp $103-105^{\circ} \mathrm{C}\left(\mathrm{CH}_{2} \mathrm{Cl}_{2}\right)$

$\mathbf{R}_{f} 0.48\left(\mathrm{CH}_{2} \mathrm{Cl}_{2} / \mathrm{MeOH}, 9: 1\right)$.

${ }^{1}$ H NMR $\left(400 \mathrm{MHz}, \mathrm{CDCl}_{3}\right): \delta(\mathrm{ppm})=8.17-8.08(\mathrm{~m}, 2 \mathrm{H}), 7.18-7.09(\mathrm{~m}, 2 \mathrm{H}), 3.09(\mathrm{~s}, 3 \mathrm{H}), 2.48$ (br. s, 2H).

${ }^{13}$ C NMR $\left(100 \mathrm{MHz}, \mathrm{CDCl}_{3}\right): \delta(\mathrm{ppm})=165.2\left(\mathrm{~d},{ }^{1} J_{\mathrm{CF}}=254.2 \mathrm{~Hz}\right), 140.4\left(\mathrm{~d},{ }^{4} J_{\mathrm{CF}}=3.1 \mathrm{~Hz}\right), 129.9$ $\left(\mathrm{d},{ }^{3} J_{\mathrm{CF}}=9.3 \mathrm{~Hz}\right), 116.1\left(\mathrm{~d},{ }^{2} J_{\mathrm{CF}}=22.4 \mathrm{~Hz}\right), 50.6$.

${ }^{19}$ F NMR $\left(376 \mathrm{MHz}, \mathrm{CDCl}_{3}\right): \delta(\mathrm{ppm})=-106.7(\mathrm{tt}, J=8.5,5.1 \mathrm{~Hz})$.

IR (ATR): $\tilde{v}\left(\mathrm{~cm}^{-1}\right)=1589,1490,1227,1057,933,839,661$.

HRMS $\left(\mathrm{ESI}^{+}\right)$calcd. for $\mathrm{C}_{7} \mathrm{H}_{10} \mathrm{FN}_{2} \mathrm{~S}^{+}[\mathrm{M}+\mathrm{H}]^{+}:$173.0543; found: 173.0543 .

\subsubsection{Deprotection of Sulfilimine}

\section{(4-Fluorophenyl)(p-tolyl)- $\lambda^{4}$-sulfaniminium-4-methylbenzenesulfonate (9)}<smiles>Cc1ccc([SH](N)c2ccc(F)cc2)cc1</smiles>

In a $25 \mathrm{~mL}$ round bottom flask, sulfilimine 3a $(225 \mathrm{mg}, 0.652 \mathrm{mmol}, 1.0$ equiv.) was dissolved in anhydrous $\mathrm{CH}_{2} \mathrm{Cl}_{2}(6.5 \mathrm{~mL})$. Triflic acid $(290 \mu \mathrm{L}, 3.29 \mathrm{mmol}, 5.0$ equiv.) was added and the 
reaction was stirred at room temperature for $1 \mathrm{~h}$. The mixture was diluted with $\mathrm{CH}_{2} \mathrm{Cl}_{2}$ and basified to $\mathrm{pH}$ 10-11 using $1 \mathrm{M}$ aq. $\mathrm{NaOH}$ solution. The separated aqueous phase was extracted with $\mathrm{CH}_{2} \mathrm{Cl}_{2}$ $(3 \times 30 \mathrm{~mL})$. The combined extracts were washed with brine, dried over anhydrous $\mathrm{Na}_{2} \mathrm{SO}_{4}$, and concentrated in vacuo. Then the crude sulfilimine was dissolved in $\mathrm{CH}_{2} \mathrm{Cl}_{2}(20 \mathrm{~mL}), \mathrm{TsOH} \cdot \mathrm{H}_{2} \mathrm{O}$ (123 mg, $0.715 \mathrm{mmol}, 1.1$ equiv.) was added and stirred at room temperature for 10 min until a homogeneous solution is formed. The mixture was then concentrated in vacuo and was purified by flash column chromatography $\left(\mathrm{CH}_{2} \mathrm{Cl}_{2} / \mathrm{MeOH}, 20: 1\right.$ to 5:1) to afford sulfiliminium salt 9 as a waxy solid (255 mg, 97\%).

$\mathbf{R}_{f} 0.62\left(\mathrm{CH}_{2} \mathrm{Cl}_{2} / \mathrm{MeOH}, 5: 1\right)$.

${ }^{1} \mathbf{H}$ NMR (400 MHz, CD $\left.{ }_{3} \mathrm{OD}\right): \delta(\mathrm{ppm})=7.87-7.81(\mathrm{~m}, 2 \mathrm{H}), 7.70-7.76(\mathrm{~m}, 4 \mathrm{H}), 7.50(\mathrm{~d}, J=8.1 \mathrm{~Hz}$, 2H), 7.45-7.39 (m, 2H), 7.19 (d, J=7.8 Hz, 2H), 2.44 (s, 3H), 2.34 (s, 3H).

${ }^{13}$ C NMR (100 MHz, CD 3 OD): $\delta(\mathrm{ppm})=166.9\left(\mathrm{~d},{ }^{1} J_{\mathrm{CF}}=255.7 \mathrm{~Hz}\right), 146.7,143.5,141.6,132.4$, $132.3\left(\mathrm{~d},{ }^{3} J_{\mathrm{CF}}=9.7 \mathrm{~Hz}\right), 130.5,130.0\left(\mathrm{~d},{ }^{4} J_{\mathrm{CF}}=3.2 \mathrm{~Hz}\right), 129.8,129.4,126.9,119.0(\mathrm{~d}$, $\left.{ }^{2} J_{\mathrm{CF}}=23.3 \mathrm{~Hz}\right), 21.5,21.3$.

${ }^{19}$ F NMR $\left(376 \mathrm{MHz}, \mathrm{CD}_{3} \mathrm{OD}\right): \delta(\mathrm{ppm})=-105.7(\mathrm{tt}, J=8.2,4.7 \mathrm{~Hz})$.

IR (ATR): $\tilde{v}\left(\mathrm{~cm}^{-1}\right)=2981,2361,2341,1589,1494,1227,1177,1122,1034,1011,814,683$.

HRMS $\left(\mathrm{ESI}^{+}\right)$calcd. for $\mathrm{C}_{13} \mathrm{H}_{13} \mathrm{FNS}^{+}[\mathrm{M}+\mathrm{H}]^{+}:$234.0747; found: 234.0746.

\subsubsection{Deprotection of Sulfoximine}

\section{(4-Fluorophenyl)(imino)(p-tolyl)- $\lambda^{6}$-sulfanone (10)}<smiles>Cc1ccc(S(=N)(=O)c2ccc(F)cc2)cc1</smiles>

Sulfoximine 5d (69 mg, $0.19 \mathrm{mmol}, 1.0$ equiv.) was mixed with TFA (1.5 mL) and heated to $60{ }^{\circ} \mathrm{C}$ for $18 \mathrm{~h}$. The mixture was concentrated in vacuo then diluted with $\mathrm{CH}_{2} \mathrm{Cl}_{2}$ and basified to $\mathrm{pH} 10-11$ using $1 \mathrm{M}$ aq. $\mathrm{NaOH}$ solution. The product was extracted with $\mathrm{CH}_{2} \mathrm{Cl}_{2}(3 \times 30 \mathrm{~mL})$. The combined extracts were washed with brine, dried over anhydrous $\mathrm{Na}_{2} \mathrm{SO}_{4}$, and concentrated in vacuo. The crude product was purified by flash column chromatography (petrol/ethyl acetate, $3: 1$ to $1: 2$ ) to afford sulfoximine $\mathbf{1 0}$ as a colourless oil (47 mg, 99\%).

$\mathbf{R}_{f} 0.41$ (petrol/ethyl acetate, 1:1). 
${ }^{1} \mathbf{H}$ NMR $\left(400 \mathrm{MHz}, \mathrm{CDCl}_{3}\right): \delta(\mathrm{ppm})=8.05-8.00(\mathrm{~m}, 2 \mathrm{H}), 7.89(\mathrm{~d}, J=8.4 \mathrm{~Hz}, 2 \mathrm{H}), 7.26(\mathrm{~d}$, $J=8.4 \mathrm{~Hz}, 2 \mathrm{H}), 7.14-7.08(\mathrm{~m}, 2 \mathrm{H}), 2.90$ (br. s, 1H), 2.37 (s, 3H).

${ }^{13}$ C NMR $\left(100 \mathrm{MHz}, \mathrm{CDCl}_{3}\right): \delta(\mathrm{ppm})=165.2\left(\mathrm{~d},{ }^{1} J_{\mathrm{CF}}=254.4 \mathrm{~Hz}\right), 143.7,140.5,139.8(\mathrm{~d}$, $\left.{ }^{4} J_{\mathrm{CF}}=3.1 \mathrm{~Hz}\right), 130.7\left(\mathrm{~d},{ }^{3} J_{\mathrm{CF}}=9.5 \mathrm{~Hz}\right), 130.0,128.0,116.4\left(\mathrm{~d},{ }^{2} J_{\mathrm{CF}}=22.7 \mathrm{~Hz}\right), 21.6$.

${ }^{19}$ F NMR $\left(376 \mathrm{MHz}, \mathrm{CDCl}_{3}\right): \delta(\mathrm{ppm})=-106.2(\mathrm{tt}, J=8.3,5.1 \mathrm{~Hz})$

IR (ATR): $\tilde{v}\left(\mathrm{~cm}^{-1}\right)=2981,2888,1588,1489,1382,1229,1130,1094,968,839$.

HRMS (ESI ${ }^{+}$) calcd. for $\mathrm{C}_{13} \mathrm{H}_{13} \mathrm{FNOS}^{+}[\mathrm{M}+\mathrm{H}]^{+}$: 250.0696; found: 250.0695.

\subsubsection{N-Functionalization of Sulfondiimine}

$N$-((4-Fluorophenyl)(methyl)(phenylimino)- $\lambda^{6}$-sulfaneylidene)-4-nitrobenzenesulfonamide (11a)<smiles></smiles>

Sulfondiimine 6 (50 mg, $0.14 \mathrm{mmol}, 1.0$ equiv.), phenylboronic acid (45 mg, $0.37 \mathrm{mmol}$, 2.5 equiv.), $\mathrm{Cu}(\mathrm{MeCN})_{4} \mathrm{PF}_{6}$ (26 mg, $0.070 \mathrm{mmol}, 0.5$ equiv.), $N$-methylpiperidine (123 mg, 1.24 mmol, 9.0 equiv.) were added to an oven-dried $10 \mathrm{~mL}$ vial and dissolved in $\mathrm{MeCN}(1.4 \mathrm{~mL})$ under oxygen atmosphere. The reaction was stirred at room temperature for $24 \mathrm{~h}$ until completion of the reaction (TLC). The reaction was quenched with water and extracted with ethyl acetate $(3 \times 20 \mathrm{~mL})$. The combined extracts were washed with brine, dried over anhydrous $\mathrm{Na}_{2} \mathrm{SO}_{4}$, and concentrated in vacuo. The crude product was purified by flash column chromatography (petrol/ethyl acetate, $4: 1$ to $2: 1$ ) to afford sulfondiimine 11a as a white solid (51 $\mathrm{mg}, 84 \%$ ).

mp $177-179^{\circ} \mathrm{C}\left(\mathrm{CH}_{2} \mathrm{Cl}_{2}\right)$

$\mathbf{R}_{f} 0.39$ (petrol/ethyl acetate, 2:1).

${ }^{1} \mathbf{H}$ NMR $\left(400 \mathrm{MHz}, \mathrm{CDCl}_{3}\right): \delta(\mathrm{ppm})=8.08-8.04(\mathrm{~m}, 2 \mathrm{H}), 7.91(\mathrm{~d}, J=8.9 \mathrm{~Hz}, 2 \mathrm{H}), 7.81(\mathrm{~d}$, $J=8.9 \mathrm{~Hz}, 2 \mathrm{H}), 7.29-7.23(\mathrm{~m}, 2 \mathrm{H}), 6.79-6.73(\mathrm{~m}, 2 \mathrm{H}), 6.69-6.63(\mathrm{~m}, 1 \mathrm{H}), 6.40-6.35$ (m, 2H), 3.76 $(\mathrm{s}, 3 \mathrm{H})$. 
${ }^{13} \mathrm{C}$ NMR $\left(100 \mathrm{MHz}, \mathrm{CDCl}_{3}\right): \delta(\mathrm{ppm})=166.3\left(\mathrm{~d},{ }^{1} J_{\mathrm{CF}}=258.6 \mathrm{~Hz}\right), 149.4,147.5,142.6,131.9(\mathrm{~d}$, $\left.{ }^{4} J_{\mathrm{CF}}=3.2 \mathrm{~Hz}\right), 131.1\left(\mathrm{~d},{ }^{3} J_{\mathrm{CF}}=9.7 \mathrm{~Hz}\right), 128.7,128.3,123.5,122.1,121.9,117.9\left(\mathrm{~d},{ }^{2} J_{\mathrm{CF}}=23.0 \mathrm{~Hz}\right)$, 49.1 .

${ }^{19}$ F NMR $\left(376 \mathrm{MHz}, \mathrm{CDCl}_{3}\right): \delta(\mathrm{ppm})=-101.9(\mathrm{tt}, J=8.0,4.8 \mathrm{~Hz})$.

IR (ATR): $\tilde{v}\left(\mathrm{~cm}^{-1}\right)=1592,1527,1487,1350,1294,1260,1156,1065,1002,797,737,691$

HRMS $\left(\mathrm{ESI}^{+}\right.$) calcd. for $\mathrm{C}_{19} \mathrm{H}_{17} \mathrm{FN}_{3} \mathrm{O}_{4} \mathrm{~S}_{2}^{+}[\mathrm{M}+\mathrm{H}]^{+}$: 434.0639; found: 434.0642 .

\section{1-(4-Fluorophenyl)-1-methyl- $N$-phenyl- $\lambda^{6}$-sulfanediimine (11)}

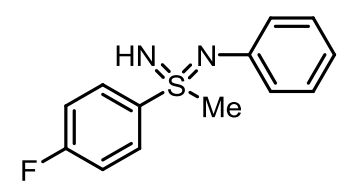

Under an atmosphere of nitrogen, sulfondiimines $\mathbf{1 1 a}(42 \mathrm{mg}, 0.10 \mathrm{mmol}, 1.0$ equiv.) was dissolved in anhydrous $\mathrm{MeCN}(1.0 \mathrm{ml})$. 1-Dodecanethiol $(0.12 \mathrm{~mL}, 0.50 \mathrm{mmol}, 5.0$ equiv.) was added at room temperature followed by DBU (70 $\mu \mathrm{L}, 0.47 \mathrm{mmol}, 4.8$ equiv.). The solution became yellow and was stirred for 20 min under nitrogen until completion of the reaction (TLC). The reaction mixture was concentrated in vacuo to afford light yellow oil. The crude product was purified by flash column chromatography (petrol/ethyl acetate, 1:1 to 0:1) to afford corresponding sulfondiimine $\mathbf{1 1}$ as a white solid (19 $\mathrm{mg}, 80 \%)$.

mp $122-123{ }^{\circ} \mathrm{C}\left(\mathrm{CH}_{2} \mathrm{Cl}_{2}\right)$

$\mathbf{R}_{f} 0.39$ (ethyl acetate).

${ }^{1} \mathbf{H}$ NMR $\left(400 \mathrm{MHz} \mathrm{CDCl}_{3}\right): \delta(\mathrm{ppm})=8.23-8.16(\mathrm{~m}, 2 \mathrm{H}), 7.25-7.14(\mathrm{~m}, 4 \mathrm{H}), 7.10-7.06(\mathrm{~m}, 2 \mathrm{H})$, $6.90(\mathrm{tt}, J=7.5,1.2 \mathrm{~Hz}, 1 \mathrm{H}), 3.25$ (s, 3H), 2.17 (br. s, 1H).

${ }^{13}$ C NMR $\left(100 \mathrm{MHz}, \mathrm{CDCl}_{3}\right): \delta(\mathrm{ppm})=165.5\left(\mathrm{~d},{ }^{1} J_{\mathrm{CF}}=254.8 \mathrm{~Hz}\right), 145.8,138.0\left(\mathrm{~d},{ }^{4} J_{\mathrm{CF}}=3.1 \mathrm{~Hz}\right)$, $130.7\left(\mathrm{~d},{ }^{3} J_{\mathrm{CF}}=9.4 \mathrm{~Hz}\right), 129.2,123.2,121.3,116.6\left(\mathrm{~d},{ }^{2} J_{\mathrm{CF}}=22.6 \mathrm{~Hz}\right), 48.6$.

${ }^{19}$ F NMR $\left(376 \mathrm{MHz}, \mathrm{CDCl}_{3}\right): \delta(\mathrm{ppm})=-106.1(\mathrm{tt}, J=8.3,5.2 \mathrm{~Hz})$.

IR (ATR): $\tilde{v}\left(\mathrm{~cm}^{-1}\right)=1590,1486,1390,1254,1153,1081,956,839,758$

HRMS $\left(\right.$ ESI $^{+}$) calcd. for $\mathrm{C}_{13} \mathrm{H}_{14} \mathrm{FN}_{2} \mathrm{~S}^{+}[\mathrm{M}+\mathrm{H}]^{+}:$249.0856; found: 249.0855 . 


\section{$N$-((Allylimino)(4-fluorophenyl)(methyl)- $\lambda^{6}$-sulfaneylidene)-4-nitrobenzenesulfonamide (12a)}

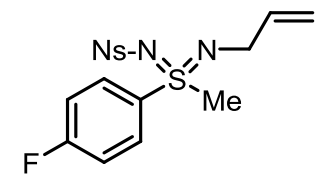

In an oven-dried flask, sulfondiimine 6 (64 $\mathrm{mg}, 0.18 \mathrm{mmol}, 1.0$ equiv.) was dissolved in anhydrous DMSO (0.40 mL). KOH (21 mg, $0.37 \mathrm{mmol}, 2.0$ equiv.) was added followed by allyl bromide ( $35 \mathrm{mg}, 0.29 \mathrm{mmol}, 1.5$ equiv.). The reaction mixture was stirred at room temperature for $12 \mathrm{~h}$ until completion of the reaction (TLC). The reaction was quenched with water and extracted with $\mathrm{CH}_{2} \mathrm{Cl}_{2}$ $(3 \times 20 \mathrm{~mL})$. The combined extracts were washed with brine, dried over anhydrous $\mathrm{Na}_{2} \mathrm{SO}_{4}$, and concentrated in vacuo. The crude product was purified by flash column chromatography (petrol/ethyl acetate, 1:1) to afford sulfondiimine 12a as a light yellow oil (58 $\mathrm{mg}, 81 \%)$.

$\mathbf{R}_{f} 0.35$ (petrol/ethyl acetate, 1:1).

${ }^{1} \mathbf{H}$ NMR $\left(400 \mathrm{MHz}, \mathrm{CDCl}_{3}\right): \delta(\mathrm{ppm})=8.30(\mathrm{~d}, J=9.0 \mathrm{~Hz}, 2 \mathrm{H}), 8.18(\mathrm{~d}, J=9.0 \mathrm{~Hz}, 2 \mathrm{H})$, 8.04-7.96 (m, 2H), 7.41-6.94 (m, 2H), 5.60 (ddt, $J=17.0,10.3,5.2 \mathrm{~Hz}, 1 \mathrm{H}), 5.05$ (dq, $J=17.0,1.8 \mathrm{~Hz}, 1 \mathrm{H}), 4.97(\mathrm{dq}, J=10.3,1.8 \mathrm{~Hz}, 1 \mathrm{H}), 3.59$ (s, 3H), $3.12(\mathrm{ddt}, J=15.5,5.2,1.7 \mathrm{~Hz}$, $1 \mathrm{H}), 2.90(\mathrm{ddt}, J=15.5,5.2,1.7 \mathrm{~Hz}, 1 \mathrm{H})$.

${ }^{13}$ C NMR $\left(100 \mathrm{MHz}, \mathrm{CDCl}_{3}\right): \delta(\mathrm{ppm})=166.2\left(\mathrm{~d},{ }^{1} J_{\mathrm{CF}}=258.0 \mathrm{~Hz}\right), 149.7,149.6,135.5,132.4(\mathrm{~d}$, $\left.{ }^{4} J_{\mathrm{CF}}=3.2 \mathrm{~Hz}\right), 131.3\left(\mathrm{~d},{ }^{3} J_{\mathrm{CF}}=9.7 \mathrm{~Hz}\right), 128.3,124.1,117.5\left(\mathrm{~d},{ }^{2} J_{\mathrm{CF}}=22.7 \mathrm{~Hz}\right), 115.6,47.6,46.4$.

${ }^{19}$ F NMR $\left(376 \mathrm{MHz}, \mathrm{CDCl}_{3}\right): \delta(\mathrm{ppm})=-102.7(\mathrm{tt}, J=8.0,4.9 \mathrm{~Hz})$.

IR (ATR): $\tilde{v}\left(\mathrm{~cm}^{-1}\right)=1526,1390,1153,1084,956$.

HRMS $\left(\mathrm{ESI}^{+}\right)$calcd. for $\mathrm{C}_{16} \mathrm{H}_{17} \mathrm{FN}_{3} \mathrm{O}_{4} \mathrm{~S}_{2}^{+}[\mathrm{M}+\mathrm{H}]^{+}$: 398.0639; found: 398.0644 .

\section{$N$-Allyl-1-(4-fluorophenyl)-1-methyl- $\lambda^{6}$-sulfanediimine (12)}

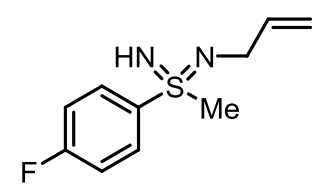

Under an atmosphere of nitrogen, sulfondiimines 12a (45 mg, $0.11 \mathrm{mmol}, 1.0$ equiv.) was dissolved in anhydrous $\mathrm{MeCN}(1.1 \mathrm{ml})$. 1-Dodecanethiol $(0.13 \mathrm{~mL}, 0.54 \mathrm{mmol}, 5.0$ equiv.) was added at room temperature followed by DBU ( $78 \mu \mathrm{L}, 0.52 \mathrm{mmol}, 4.75$ equiv.). The solution became yellow 
and was stirred for 20 mins under nitrogen until completion of the reaction (TLC). The reaction mixture was concentrated in vacuo to afford light yellow oil. The crude product was purified by flash column chromatography $\left(\mathrm{CH}_{2} \mathrm{Cl}_{2} / \mathrm{MeOH}, 15: 1\right.$ to 9:1) to afford corresponding sulfondiimine 12 as a light yellow oil (22 mg, 94\%).

$\mathbf{R}_{f} 0.43\left(\mathrm{CH}_{2} \mathrm{Cl}_{2} / \mathrm{MeOH}=9: 1\right)$.

${ }^{1} \mathbf{H}$ NMR $\left(400 \mathrm{MHz}, \mathrm{CDCl}_{3}\right): \delta(\mathrm{ppm})=8.16-8.01(\mathrm{~m}, 2 \mathrm{H}), 7.23-7.17$ (m, 2H), $5.96(\mathrm{ddt}, J=17.0$, $10.1,5.6 \mathrm{~Hz}, 1 \mathrm{H}), 5.25(\mathrm{dq}, J=17.0,1.8 \mathrm{~Hz}, 1 \mathrm{H}), 5.05(\mathrm{dq}, J=10.1,1.8 \mathrm{~Hz}, 1 \mathrm{H}), 3.68$ (ddt, $J=15.2,5.6,1.6 \mathrm{~Hz}, 1 \mathrm{H}), 3.53(\mathrm{ddt}, J=15.2,5.6,1.6 \mathrm{~Hz}, 1 \mathrm{H}), 3.12$ (s, 3H), 2.25 (br. s, $1 \mathrm{H})$.

${ }^{13} \mathrm{C}$ NMR $\left(100 \mathrm{MHz}, \mathrm{CDCl}_{3}\right): \delta(\mathrm{ppm})=165.3\left(\mathrm{~d},{ }^{1} J_{\mathrm{CF}}=254.1 \mathrm{~Hz}\right), 138.5,137.6\left(\mathrm{~d},{ }^{4} J_{\mathrm{CF}}=3.0 \mathrm{~Hz}\right)$, $130.6\left(\mathrm{~d},{ }^{3} J_{\mathrm{CF}}=9.3 \mathrm{~Hz}\right), 116.4\left(\mathrm{~d},{ }^{2} J_{\mathrm{CF}}=22.5 \mathrm{~Hz}\right), 114.8,48.5,45.7$.

${ }^{19}$ F NMR $\left(376 \mathrm{MHz}, \mathrm{CDCl}_{3}\right): \delta(\mathrm{ppm})=-106.7(\mathrm{tt}, J=8.3,5.1 \mathrm{~Hz})$

IR (ATR): $\tilde{v}\left(\mathrm{~cm}^{-1}\right)=1490,1389,1253,1153,1076,956$.

HRMS $\left(\mathrm{ESI}^{+}\right.$) calcd. for $\mathrm{C}_{10} \mathrm{H}_{14} \mathrm{FN}_{2} \mathrm{~S}^{+}[\mathrm{M}+\mathrm{H}]^{+}: 213.0856$; found: 213.0860.

\section{$N$-((4-Fluorophenyl)(methyl)((2,4,4-trimethylpentan-2-yl)imino)- $\lambda^{6}$-sulfaneylidene)acetamide}

(13a)<smiles>CCON=S(C)(=NC(=O)OC)c1ccc(F)cc1</smiles>

Under an atmosphere of argon, sulfondiimine 7 (40 mg, $0.14 \mathrm{mmol}, 1.0$ equiv.) was dissolved in anhydrous $\mathrm{CH}_{2} \mathrm{Cl}_{2}(1.5 \mathrm{~mL})$. Triethylamine $\left(30 \mu \mathrm{L}, 0.22 \mathrm{mmol}, 1.5\right.$ equiv.) was added at $0^{\circ} \mathrm{C}$ followed by acetic anhydride $(20 \mu \mathrm{L}, 0.21 \mathrm{mmol}, 1.5$ equiv.) and DMAP (4.1 mg, $0.034 \mathrm{mmol}$, 0.25 equiv.). The reaction mixture was stirred at room temperature for $12 \mathrm{~h}$. Sat. aq. $\mathrm{NH}_{4} \mathrm{Cl}(30 \mathrm{~mL})$ was added and the product was extracted with $\mathrm{CH}_{2} \mathrm{Cl}_{2}(3 \times 10 \mathrm{~mL})$. The combined extracts were washed with brine, dried over anhydrous $\mathrm{Na}_{2} \mathrm{SO}_{4}$, and concentrated in vacuo. The crude product was purified by flash column chromatography (petrol/ethyl acetate, 1.5:1) to afford sulfondiimine 13a as a white solid (45 mg, 99\%).

$m p 64-65^{\circ} \mathrm{C}\left(\mathrm{CH}_{2} \mathrm{Cl}_{2}\right)$

$\mathbf{R}_{f} 0.37$ (petrol/ethyl acetate, 1.5:1). 
${ }^{1} \mathbf{H}$ NMR $\left(400 \mathrm{MHz}, \mathrm{CDCl}_{3}\right): \delta(\mathrm{ppm})=8.12-8.02(\mathrm{~m}, 2 \mathrm{H}), 7.23-7.12(\mathrm{~m}, 2 \mathrm{H}), 3.36(\mathrm{~s}, 3 \mathrm{H}), 2.12(\mathrm{~s}$, 3H), $1.52(\mathrm{~d}, J=14.4 \mathrm{~Hz}, 1 \mathrm{H}), 1.47$ (d, $J=14.4 \mathrm{~Hz}, 1 \mathrm{H}), 1.32(\mathrm{~s}, 3 \mathrm{H}), 1.13(\mathrm{~s}, 3 \mathrm{H}), 1.02(\mathrm{~s}, 9 \mathrm{H})$.

${ }^{13}$ C NMR $\left(100 \mathrm{MHz},\left(\mathrm{CD}_{3}\right)_{2} \mathrm{CO}\right): \delta(\mathrm{ppm})=179.6,166.0\left(\mathrm{~d},{ }^{1} J_{\mathrm{CF}}=251.7 \mathrm{~Hz}\right), 141.5(\mathrm{~d}$, $\left.{ }^{4} J_{\mathrm{CF}}=3.2 \mathrm{~Hz}\right), 131.6\left(\mathrm{~d},{ }^{3} J_{\mathrm{CF}}=9.1 \mathrm{~Hz}\right), 117.0\left(\mathrm{~d},{ }^{2} J_{\mathrm{CF}}=23.0 \mathrm{~Hz}\right), 59.6,59.0,47.9,32.7,32.6,32.4$, $32.2,27.5$.

${ }^{19} \mathbf{F ~ N M R}\left(376 \mathrm{MHz}, \mathrm{CDCl}_{3}\right): \delta(\mathrm{ppm})=-106.8(\mathrm{tt}, J=8.1,5.0 \mathrm{~Hz})$.

IR (ATR): $\tilde{v}\left(\mathrm{~cm}^{-1}\right)=2980,1623,1383,1360,1262,1217,1153,1079,1022,969,817$

HRMS (ESI ${ }^{+}$) calcd. for $\mathrm{C}_{17} \mathrm{H}_{28} \mathrm{FN}_{2} \mathrm{OS}^{+}[\mathrm{M}+\mathrm{H}]^{+}$: 327.1901; found: 327.1897 .

\section{$N$-((4-Fluorophenyl)(imino)(methyl)- $\lambda^{6}$-sulfaneylidene)acetamide (13)}<smiles>CN=S(N)(=O)c1ccc(F)cc1</smiles>

Sulfondiimine 13a (91 mg, $0.28 \mathrm{mmol}, 1.0$ equiv.) was mixed with TFA (2.8 mL) and stirred at room temperature for $14 \mathrm{~h}$. The mixture was concentrated in vacuo then diluted with $\mathrm{CH}_{2} \mathrm{Cl}_{2}$ and basified to $\mathrm{pH}$ 10-11 using $1 \mathrm{M}$ aq. $\mathrm{NaOH}$ solution. The product was extracted with $\mathrm{CH}_{2} \mathrm{Cl}_{2}$ $(3 \times 20 \mathrm{~mL})$. The combined extracts were washed with brine, dried over anhydrous $\mathrm{Na}_{2} \mathrm{SO}_{4}$, and concentrated in vacuo. The crude product was purified by flash column chromatography (ethyl acetate, then $\left.\mathrm{CH}_{2} \mathrm{Cl}_{2} / \mathrm{MeOH}, 5: 1\right)$ to afford sulfondiimine 13 as a colourless oil (54 mg, 90\%).

$\mathbf{R}_{f} 0.28$ (ethyl acetate).

${ }^{1} \mathbf{H}$ NMR $\left(400 \mathrm{MHz}, \mathrm{CDCl}_{3}\right): \delta(\mathrm{ppm})=8.01-7.93(\mathrm{~m}, 2 \mathrm{H})$, 7.24-7.17 (m, 2H), $3.16(\mathrm{~s}, 3 \mathrm{H}), 3.04$ (br. s, 1H), 2.07 (s, 3H).

${ }^{13}$ C NMR $\left(100 \mathrm{MHz}, \mathrm{CDCl}_{3}\right): \delta(\mathrm{ppm})=181.8,165.6\left(\mathrm{~d},{ }^{1} J_{\mathrm{CF}}=255.6 \mathrm{~Hz}\right), 137.6\left(\mathrm{~d},{ }^{4} J_{\mathrm{CF}}=3.1 \mathrm{~Hz}\right)$, $129.7\left(\mathrm{~d},{ }^{3} J_{\mathrm{CF}}=9.4 \mathrm{~Hz}\right), 117.0\left(\mathrm{~d},{ }^{2} J_{\mathrm{CF}}=22.9 \mathrm{~Hz}\right), 45.1,26.0$.

${ }^{19}$ F NMR $\left(376 \mathrm{MHz}, \mathrm{CDCl}_{3}\right): \delta(\mathrm{ppm})=-105.0(\mathrm{tt}, J=8.0,5.1 \mathrm{~Hz})$.

IR (ATR): $\tilde{v}\left(\mathrm{~cm}^{-1}\right)=1587,1490,1365,1281,1233,1157,1083,1047,944,818$

HRMS (ESI ${ }^{+}$) calcd. for $\mathrm{C}_{9} \mathrm{H}_{12} \mathrm{FN}_{2} \mathrm{OS}^{+}[\mathrm{M}+\mathrm{H}]^{+}: 215.0649$; found: 215.0648 . 


\section{1-((4-Fluorophenyl)(methyl)((2,4,4-trimethylpentan-2-yl)imino)- $\lambda^{6}$-sulfaneylidene)-3-}

phenylurea (14a)<smiles>O=C(N=S(=O)([O-])c1ccc(F)cc1)Nc1ccccc1</smiles>

To a solution of sulfondiimine 7 (38 mg, $0.13 \mathrm{mmol}, 1.0$ equiv.) in anhydrous $\mathrm{CH}_{2} \mathrm{Cl}_{2}(1.3 \mathrm{~mL})$, the phenyl isocyanate ( $26 \mathrm{mg}, 0.22 \mathrm{mmol}, 1.5$ equiv.) was added dropwise at room temperature under argon atmosphere. The reaction was stirred at room temperature for $24 \mathrm{~h}$ until completion of the reaction (TLC). The reaction was quenched with water and extracted with $\mathrm{CH}_{2} \mathrm{Cl}_{2}(3 \times 20 \mathrm{~mL})$. The combined extracts were washed with brine, dried over anhydrous $\mathrm{Na}_{2} \mathrm{SO}_{4}$, and concentrated in vacuo. The crude product was purified by flash column chromatography (petrol/ethyl acetate, 3:1 to 2:1) to afford sulfondiimine $\mathbf{1 4 a}$ as a white solid (53 $\mathrm{mg}, 98 \%$ ).

$\boldsymbol{m p} 82-84{ }^{\circ} \mathrm{C}\left(\mathrm{CH}_{2} \mathrm{Cl}_{2}\right)$

$\mathbf{R}_{f} 0.56$ (petrol/ethyl acetate, 2:1).

${ }^{1} \mathbf{H}$ NMR $\left(400 \mathrm{MHz} \mathrm{CDCl}_{3}\right): \delta(\mathrm{ppm})=8.15-8.08(\mathrm{~m}, 2 \mathrm{H}), 7.48(\mathrm{dd}, J=8.6,1.2 \mathrm{~Hz}, 1 \mathrm{H}), 7.27(\mathrm{dd}$, $J=8.5,7.3 \mathrm{~Hz}, 1 \mathrm{H}), 7.24-7.18(\mathrm{~m}, 2 \mathrm{H}), 6.99$ (tt, $J=7.3,1.2 \mathrm{~Hz}, 1 \mathrm{H}), 6.90$ (br. s, 1H), 3.43 (s, 3H), $1.56(\mathrm{~d}, J=14.3 \mathrm{~Hz}, 1 \mathrm{H}), 1.51(\mathrm{~d}, J=14.3 \mathrm{~Hz}, 1 \mathrm{H}), 1.37(\mathrm{~s}, 3 \mathrm{H}), 1.17(\mathrm{~s}, 3 \mathrm{H}), 1.04(\mathrm{~s}, 9 \mathrm{H})$.

${ }^{13} \mathrm{C}$ NMR $\left(100 \mathrm{MHz}, \mathrm{CDCl}_{3}\right): \delta(\mathrm{ppm})=165.1\left(\mathrm{~d},{ }^{1} J_{\mathrm{CF}}=254.2 \mathrm{~Hz}\right), 159.2,140.1\left(\mathrm{~d},{ }^{4} J_{\mathrm{CF}}=3.1 \mathrm{~Hz}\right)$, 139.9, 130. $2\left(\mathrm{~d},{ }^{3} J_{\mathrm{CF}}=9.4 \mathrm{~Hz}\right), 129.0,122.5,118.7,116.4\left(\mathrm{~d},{ }^{2} J_{\mathrm{CF}}=22.8 \mathrm{~Hz}\right), 59.4,58.2,48.8,32.1$, $32.0,31.92,31.89$.

${ }^{19}$ F NMR $\left(376 \mathrm{MHz}, \mathrm{CDCl}_{3}\right): \delta(\mathrm{ppm})=-106.9 .(\mathrm{ddd}, J=13.3,8.5,5.1 \mathrm{~Hz})$.

IR (ATR): $\tilde{v}\left(\mathrm{~cm}^{-1}\right)=1650,1506,1389,1217,1153,1088,962,836$.

HRMS (ESI ${ }^{+}$) calcd. for $\mathrm{C}_{22} \mathrm{H}_{31} \mathrm{FN}_{3} \mathrm{OS}^{+}[\mathrm{M}+\mathrm{H}]^{+}:$404.2166; found: 404.1273.

\section{1-((4-Fluorophenyl)(imino)(methyl)- $\lambda^{6}$-sulfaneylidene)-3-phenylurea (14)}<smiles>[N+]S(=N)(=NC(=O)Nc1ccccc1)c1ccc(F)cc1</smiles> 
Sulfondiimine 14a (50 mg, $0.12 \mathrm{mmol}, 1.0$ equiv.) was mixed with TFA (1.2 mL) and stirred at room temperature for 3 days. The mixture was concentrated in vacuo then diluted with $\mathrm{CH}_{2} \mathrm{Cl}_{2}$ and basified to $\mathrm{pH}$ 10-11 using $1 \mathrm{M}$ aq. $\mathrm{NaOH}$ solution. The product was extracted with $\mathrm{CH}_{2} \mathrm{Cl}_{2}$ $(3 \times 20 \mathrm{~mL})$. The combined extracts were washed with brine, dried over anhydrous $\mathrm{Na}_{2} \mathrm{SO}_{4}$, and concentrated in vacuo. The crude product was purified by flash column chromatography (ethyl acetate) to afford sulfondiimine $\mathbf{1 4}$ as a light yellow solid (31 $\mathrm{mg}, 86 \%)$.

\section{$m p 55-57{ }^{\circ} \mathrm{C}\left(\mathrm{CH}_{2} \mathrm{Cl}_{2}\right)$}

$\mathbf{R}_{f} 0.41$ (ethyl acetate).

${ }^{1} \mathbf{H}$ NMR $\left(400 \mathrm{MHz}, \mathrm{CDCl}_{3}\right): \delta(\mathrm{ppm})=8.07-8.01(\mathrm{~m}, 2 \mathrm{H}), 7.37(\mathrm{dd}, J=8.6,1.2 \mathrm{~Hz}, 2 \mathrm{H}), 7.26-7.18$ (m, 4H), 7.01 (br. s, 1H), 7.00-6.96 (m, 1H), 3.24 (s, 3H), 2.87 (br. s, 1H).

${ }^{13}$ C NMR $\left(100 \mathrm{MHz}, \mathrm{CDCl}_{3}\right): \delta(\mathrm{ppm})=165.7\left(\mathrm{~d},{ }^{1} J_{\mathrm{CF}}=255.5 \mathrm{~Hz}\right), 160.3,139.3,138.3(\mathrm{~d}$, $\left.{ }^{4} J_{\mathrm{CF}}=3.2 \mathrm{~Hz}\right), 130.0\left(\mathrm{~d},{ }^{3} J_{\mathrm{CF}}=9.5 \mathrm{~Hz}\right), 129.0,122.8,118.8,117.0\left(\mathrm{~d},{ }^{2} J_{\mathrm{CF}}=22.7 \mathrm{~Hz}\right), 45.7$.

${ }^{19}$ F NMR $\left(376 \mathrm{MHz}, \mathrm{CDCl}_{3}\right): \delta(\mathrm{ppm})=-105.2(\mathrm{ddd}, J=13.2,8.3,4.9 \mathrm{~Hz})$.

IR (ATR): $\tilde{v}\left(\mathrm{~cm}^{-1}\right)=1591,1535,1491,1438,1389,1317,1236,1154,1075,960,832$.

HRMS (ESI ${ }^{+}$) calcd. for $\mathrm{C}_{14} \mathrm{H}_{15} \mathrm{FN}_{3} \mathrm{OS}^{+}[\mathrm{M}+\mathrm{H}]^{+}:$292.0914; found: 292.0915 .

\section{1-(4-Fluorophenyl)-1-methyl- $N$-(4-nitrobenzyl)- $N$-(2,4,4-trimethylpentan-2-yl)- $\lambda^{6}$ -} sulfanediimine (15a)

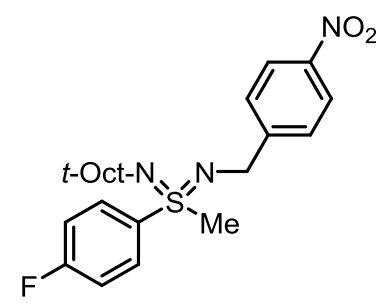

Under an atmosphere of argon, sulfondiimine 7 (91 $\mathrm{mg}, 0.32 \mathrm{mmol}, 1.0$ equiv.) was dissolved in anhydrous DCE $(1.5 \mathrm{~mL}) .4 \AA$ Molecular sieves $(0.1 \mathrm{~g})$ were added followed by 4-nitrobenzaldehyde (71 mg, $0.47 \mathrm{mmol}, 1.3$ equiv.) and $\mathrm{NaBH}(\mathrm{OAc})_{3}$ (199 mg, $0.940 \mathrm{mmol}$, 3.0 equiv.). The reaction mixture was stirred at $50{ }^{\circ} \mathrm{C}$ for $18 \mathrm{~h} .1 \mathrm{M}$ aq. $\mathrm{NaOH}$ solution $(30 \mathrm{~mL})$ was added, the product was extracted with $\mathrm{CH}_{2} \mathrm{Cl}_{2}(3 \times 30 \mathrm{~mL})$. The combined extracts were washed with brine, dried over anhydrous $\mathrm{Na}_{2} \mathrm{SO}_{4}$, and concentrated in vacuo. The crude product was 
purified by flash column chromatography (petrol/ethyl acetate, 4:1 to 1.5:1) to afford sulfondiimine 15a as a colourless oil (109 $\mathrm{mg}, 81 \%)$.

$\mathbf{R}_{f} 0.38$ (petrol/ethyl acetate, 1.5:1).

${ }^{1} \mathbf{H}$ NMR $\left(400 \mathrm{MHz}, \mathrm{CDCl}_{3}\right): \delta(\mathrm{ppm})=8.15(\mathrm{~d}, J=8.8 \mathrm{~Hz}, 2 \mathrm{H}), 8.08-8.01(\mathrm{~m}, 2 \mathrm{H}), 7.55(\mathrm{~d}$, $J=8.8 \mathrm{~Hz}, 2 \mathrm{H}), 7.19-7.10(\mathrm{~m}, 2 \mathrm{H}), 4.33(\mathrm{~d}, J=16.2 \mathrm{~Hz}, 1 \mathrm{H}), 4.08(\mathrm{~d}, J=16.2 \mathrm{~Hz}, 1 \mathrm{H}), 3.15(\mathrm{~s}$, $3 \mathrm{H}), 1.57(\mathrm{~s}, 2 \mathrm{H}), 1.46(\mathrm{~s}, 3 \mathrm{H}), 1.36(\mathrm{~s}, 3 \mathrm{H}), 1.05(\mathrm{~s}, 9 \mathrm{H})$.

${ }^{13} \mathrm{C}$ NMR $\left(100 \mathrm{MHz}, \mathrm{CDCl}_{3}\right): \delta(\mathrm{ppm})=165.2\left(\mathrm{~d},{ }^{1} J_{\mathrm{CF}}=254.5 \mathrm{~Hz}\right), 149.1,146.9,137.1(\mathrm{~d}$, $\left.{ }^{4} J_{\mathrm{CF}}=2.6 \mathrm{~Hz}\right), 131.0\left(\mathrm{~d},{ }^{3} J_{\mathrm{CF}}=9.2 \mathrm{~Hz}\right), 128.3,123.6,116.5\left(\mathrm{~d},{ }^{2} J_{\mathrm{CF}}=22.4 \mathrm{~Hz}\right), 58.9,58.5,48.2,46.2$, $32.6,32.3,32.04,32.01$.

${ }^{19}$ F NMR $\left(376 \mathrm{MHz}, \mathrm{CDCl}_{3}\right): \delta(\mathrm{ppm})=-108.2(\mathrm{ddd}, J=13.4,8.4,5.1 \mathrm{~Hz})$.

IR (ATR): $\tilde{v}\left(\mathrm{~cm}^{-1}\right)=1518,1487,1343,1216,1146,1106,839,738$.

HRMS $\left(\mathrm{ESI}^{+}\right.$) calcd. for $\mathrm{C}_{22} \mathrm{H}_{31} \mathrm{FN}_{3} \mathrm{O}_{2} \mathrm{~S}^{+}[\mathrm{M}+\mathrm{H}]^{+}$: 420.2116; found: 420.2114 .

\section{1-(4-Fluorophenyl)-1-methyl- $N$-(4-nitrobenzyl)- $\lambda^{6}$-sulfanediimine (15)}

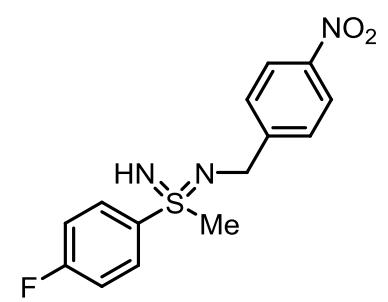

Sulfondiimine 15a (45 mg, $0.11 \mathrm{mmol}, 1.0$ equiv.) was mixed with TFA (1.1 mL) and stir at room temperature for $6 \mathrm{~h}$. The mixture was concentrated in vacuo then diluted with ethyl acetate and basified to $\mathrm{pH} 10-11$ using $1 \mathrm{M}$ aq. $\mathrm{NaOH}$ solution. The product was extracted with ethyl acetate $(3 \times 20 \mathrm{~mL})$. The combined extracts were washed with brine, dried over anhydrous $\mathrm{Na}_{2} \mathrm{SO}_{4}$, and concentrated in vacuo. The crude product was purified by flash column chromatography $\left(\mathrm{CH}_{2} \mathrm{Cl}_{2} / \mathrm{MeOH}, 20: 1\right)$ to afford sulfondiimine 15 as a light yellow solid (29 $\left.\mathrm{mg}, 88 \%\right)$.

$m p 131-133{ }^{\circ} \mathrm{C}\left(\mathrm{CH}_{2} \mathrm{Cl}_{2}\right)$

$\mathbf{R}_{f} 0.36\left(\mathrm{CH}_{2} \mathrm{Cl}_{2} / \mathrm{MeOH}, 9: 1\right)$. 
${ }^{1} \mathbf{H}$ NMR $\left(400 \mathrm{MHz}, \mathrm{CDCl}_{3}\right): \delta(\mathrm{ppm})=8.15(\mathrm{~d}, J=8.7 \mathrm{~Hz}, 2 \mathrm{H}), 8.13-8.08(\mathrm{~m}, 2 \mathrm{H}), 7.55(\mathrm{~d}$, $J=8.7 \mathrm{~Hz}, 2 \mathrm{H}), 7.25-7.17(\mathrm{~m}, 2 \mathrm{H}), 4.33(\mathrm{~d}, J=15.7 \mathrm{~Hz}, 1 \mathrm{H}), 4.18(\mathrm{~d}, J=15.7 \mathrm{~Hz}, 1 \mathrm{H}), 3.15(\mathrm{~s}$, 3H), 1.87 (br. s, 1H).

${ }^{13}$ C NMR $\left(100 \mathrm{MHz}, \mathrm{CDCl}_{3}\right): \delta(\mathrm{ppm})=165.4\left(\mathrm{~d},{ }^{1} J_{\mathrm{CF}}=254.8 \mathrm{~Hz}\right), 150.0,146.8,137.2(\mathrm{~d}$, $\left.{ }^{4} J_{\mathrm{CF}}=3.0 \mathrm{~Hz}\right), 130.6\left(\mathrm{~d},{ }^{3} J_{\mathrm{CF}}=9.4 \mathrm{~Hz}\right), 128.3,123.7,116.6\left(\mathrm{~d},{ }^{2} J_{\mathrm{CF}}=22.4 \mathrm{~Hz}\right), 48.6,46.3$.

${ }^{19}$ F NMR $\left(376 \mathrm{MHz}, \mathrm{CDCl}_{3}\right): \delta(\mathrm{ppm})=-106.0(\mathrm{ddd}, J=13.3,8.2,5.1 \mathrm{~Hz})$.

IR (ATR): $\tilde{v}\left(\mathrm{~cm}^{-1}\right)=1516,1344,1153,839$.

HRMS (ESI ${ }^{+}$) calcd. for $\mathrm{C}_{14} \mathrm{H}_{15} \mathrm{FN}_{3} \mathrm{O}_{2} \mathrm{~S}^{+}[\mathrm{M}+\mathrm{H}]^{+}$: 308.0864; found: 308.0858 . 


\section{NMR Spectra}

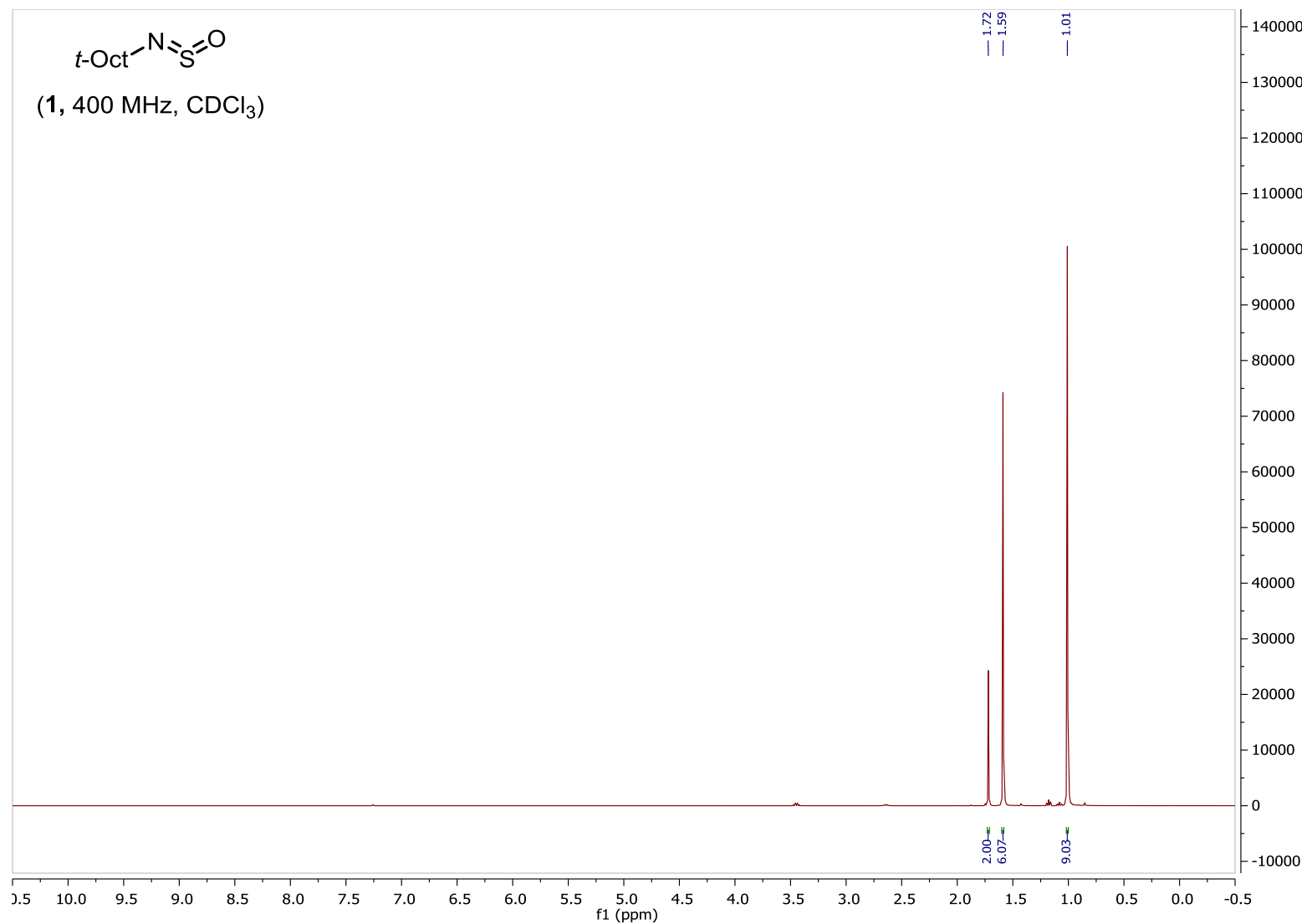

$t-\mathrm{Oct}^{-}-\mathrm{N}=\mathrm{O}$

$\left(1,100 \mathrm{MHz}, \mathrm{CDCl}_{3}\right)$

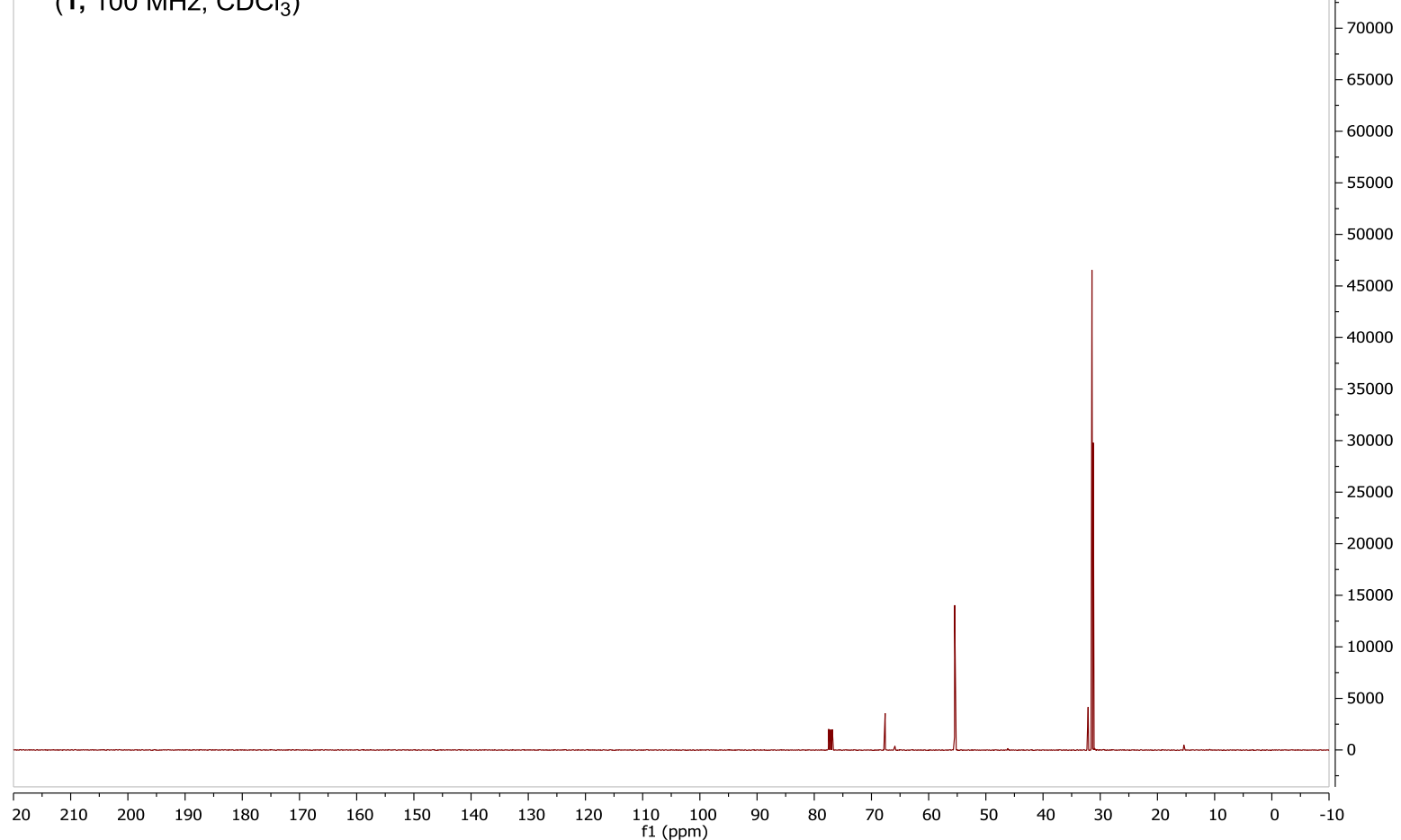




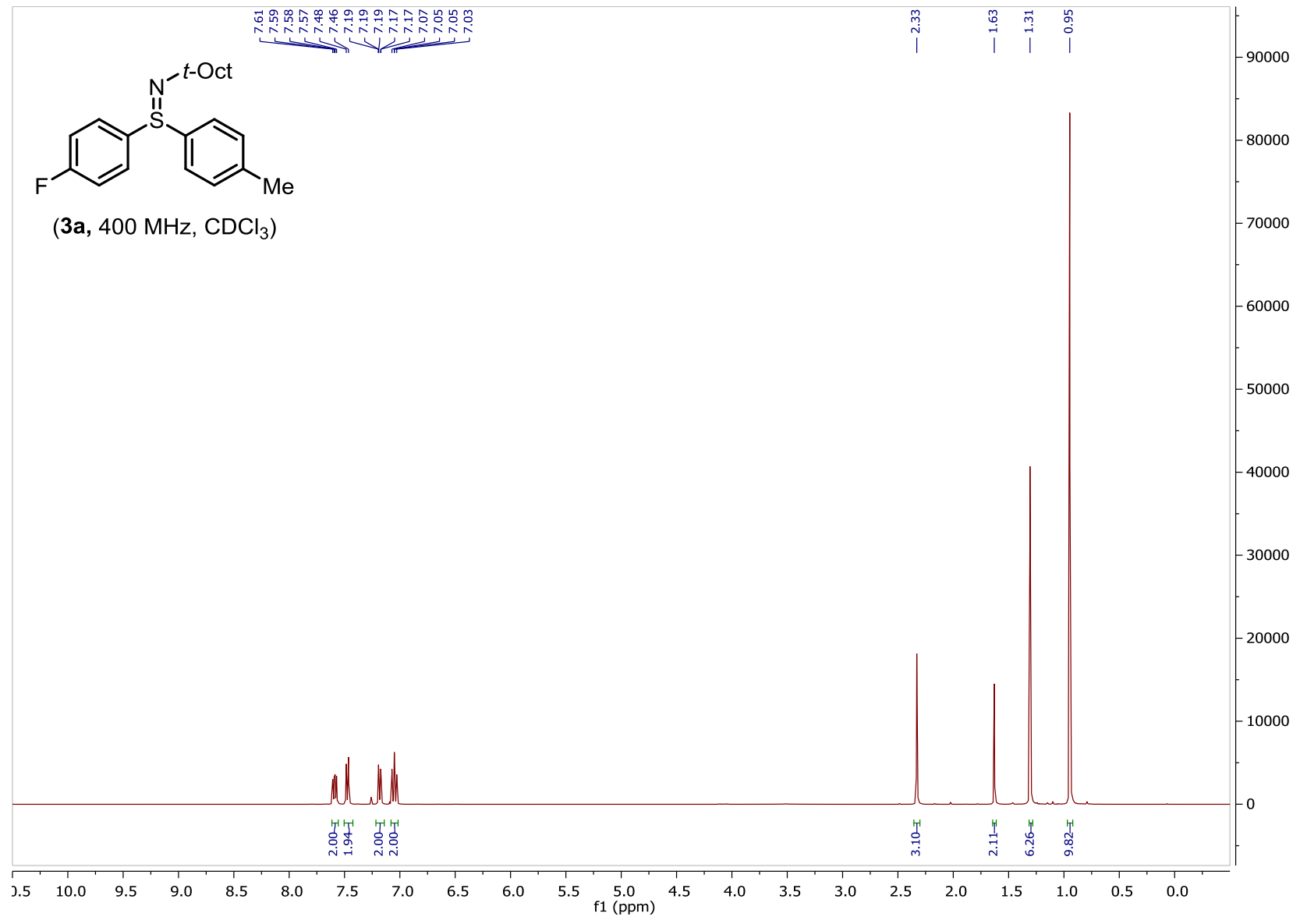<smiles>CCON=S(c1ccc(C)cc1)c1ccc(F)cc1</smiles>

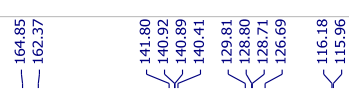

(3a, $100 \mathrm{MHz}, \mathrm{CDCl}_{3}$ )

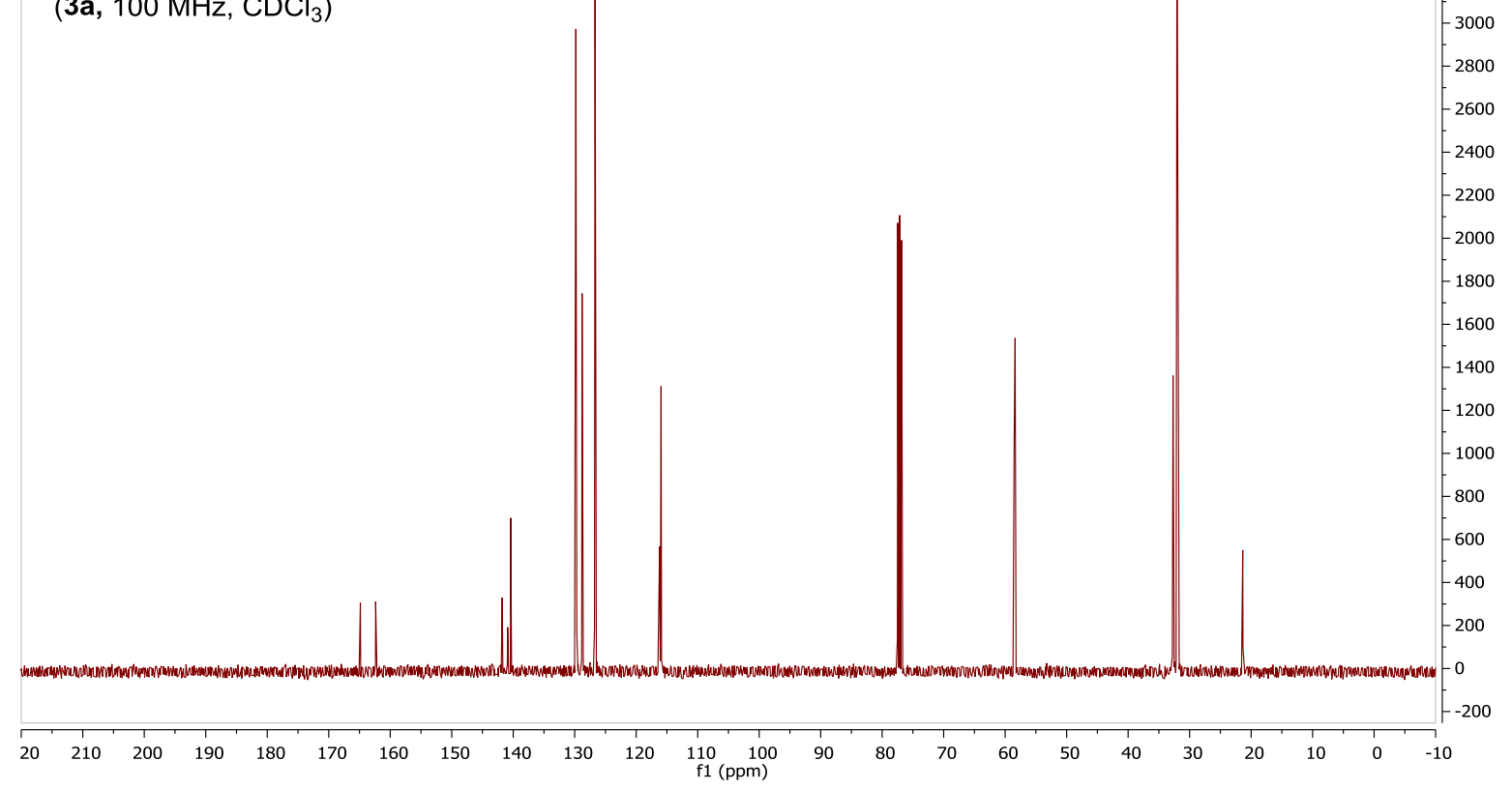




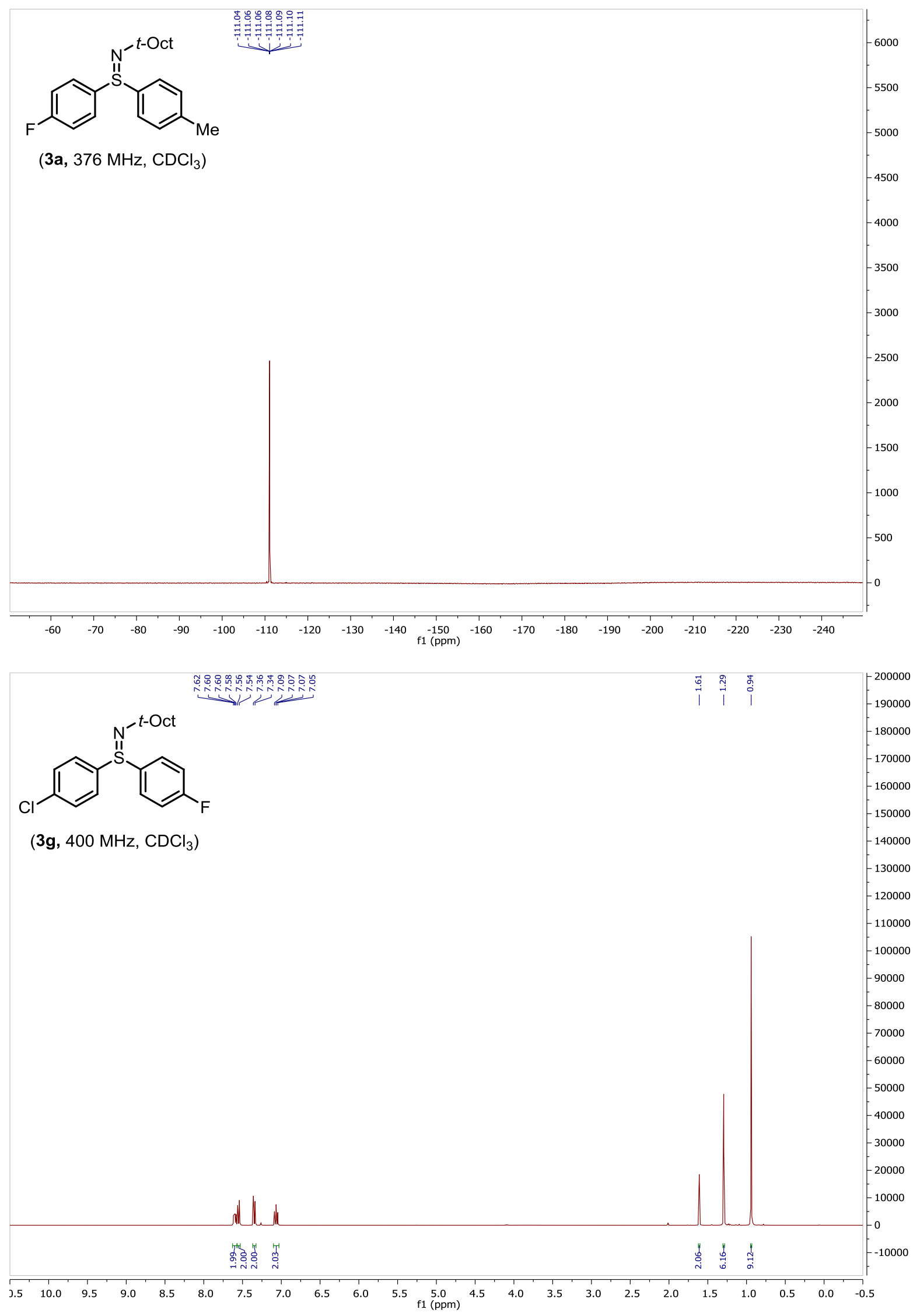



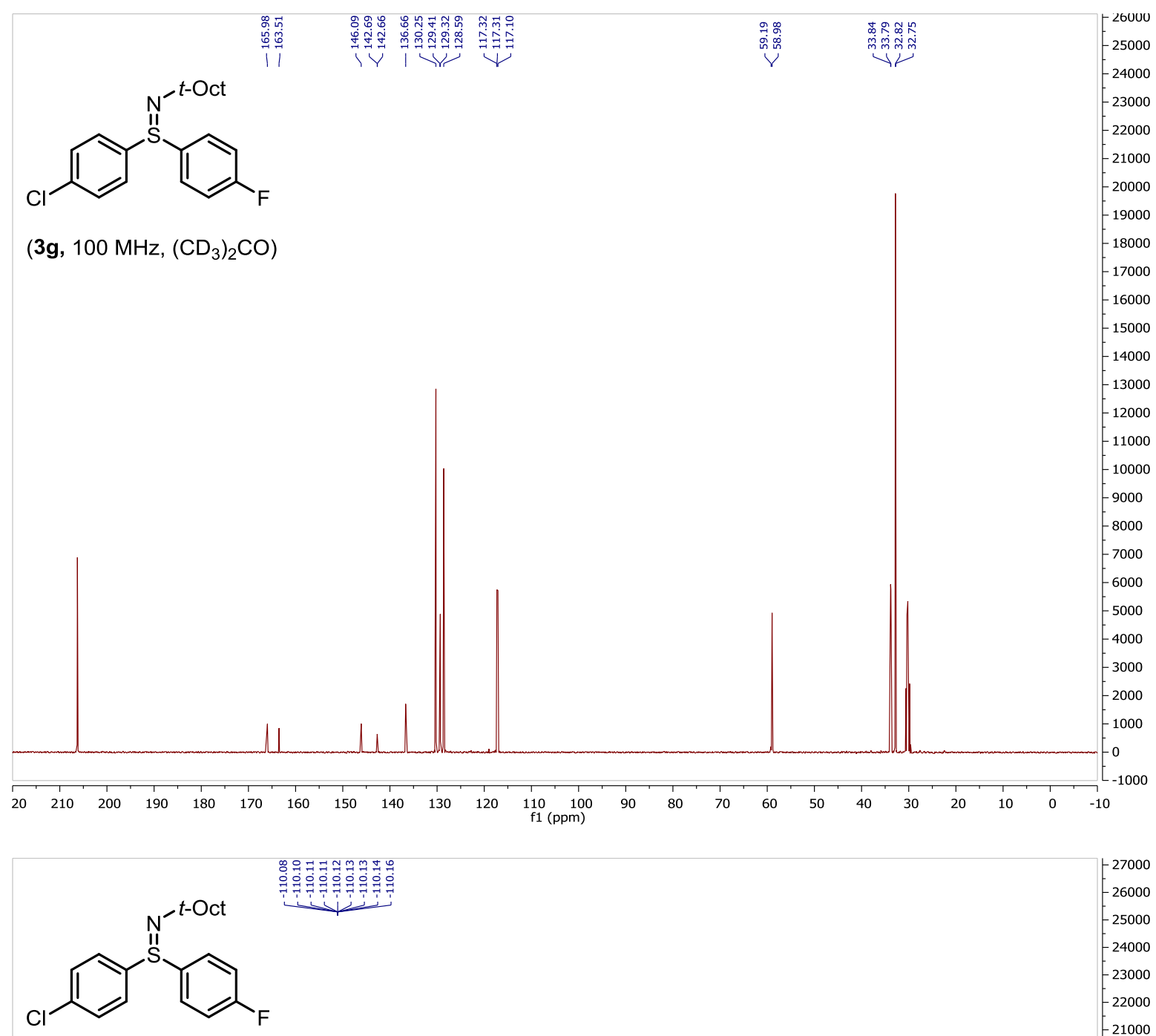

(3g, $376 \mathrm{MHz}, \mathrm{CDCl}_{3}$ )

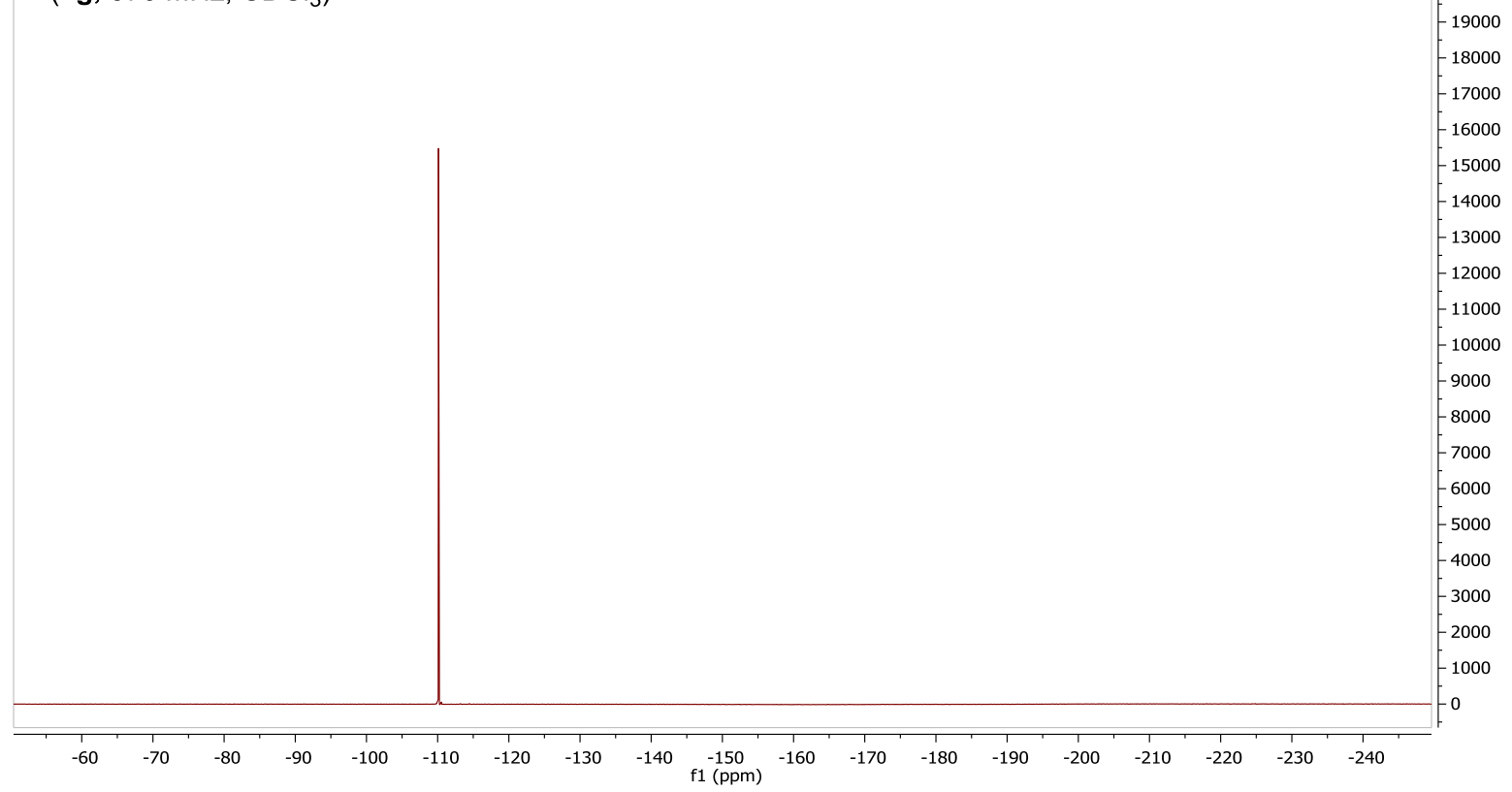




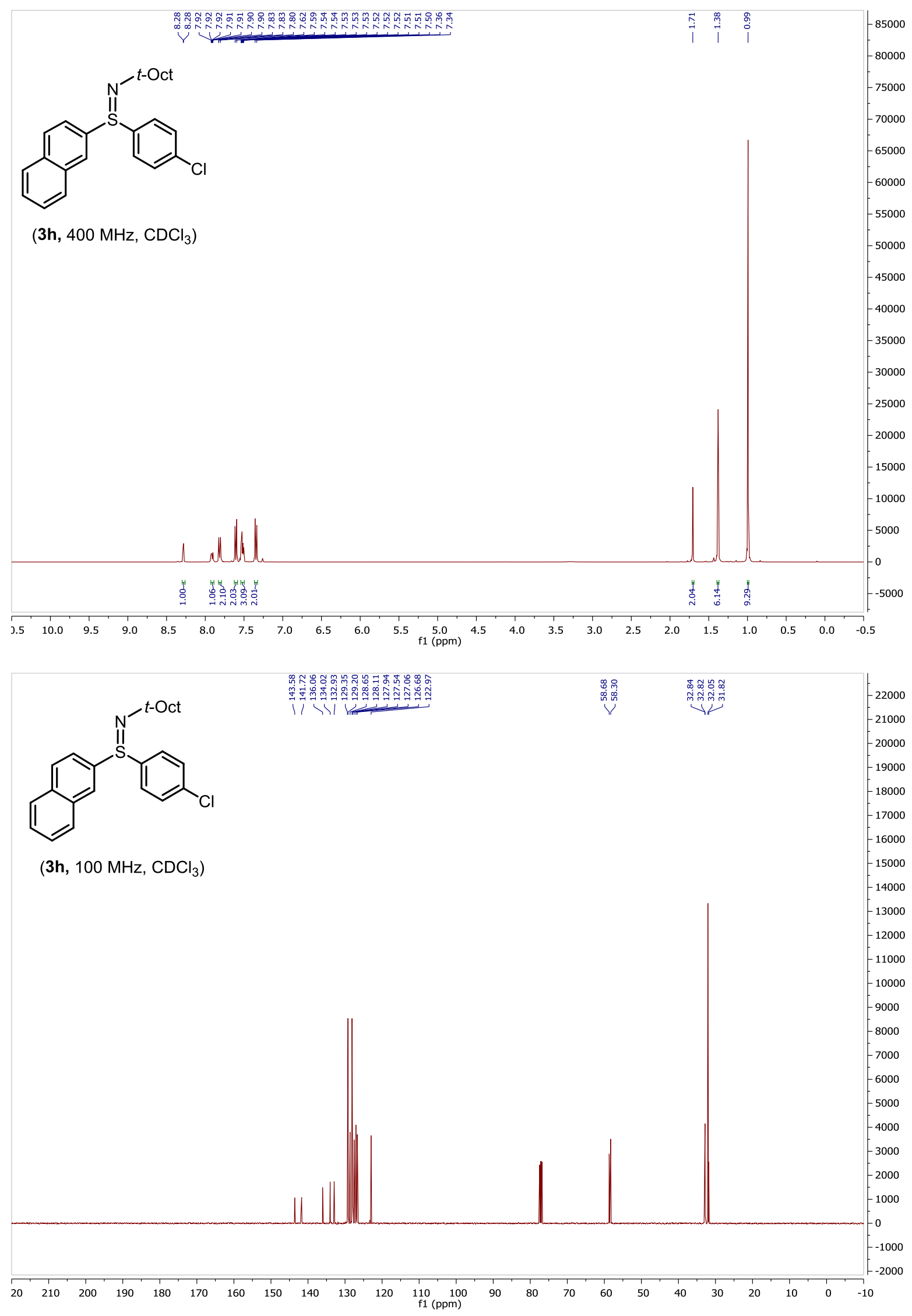



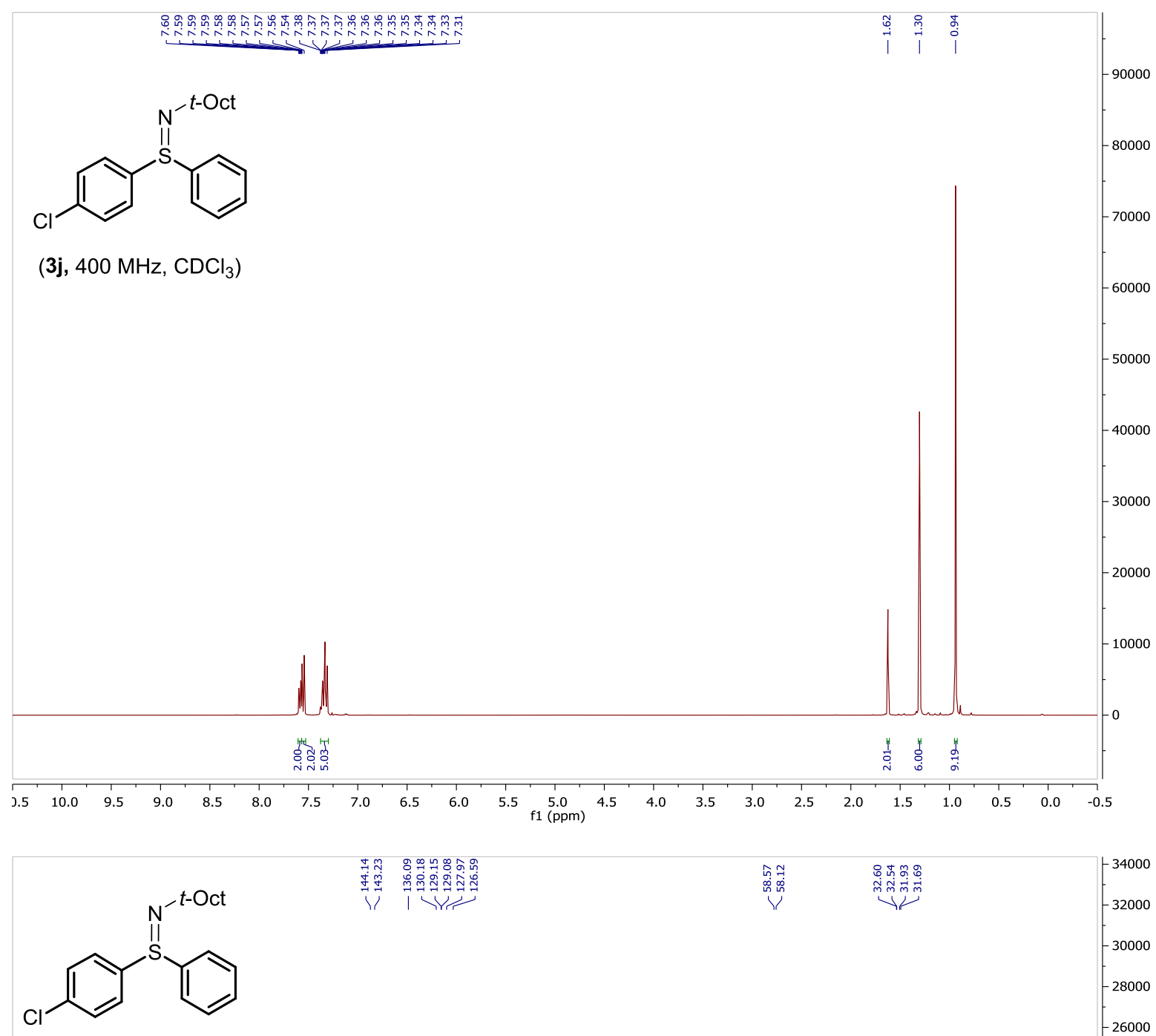

(3j, $100 \mathrm{MHz}, \mathrm{CDCl}_{3}$ )

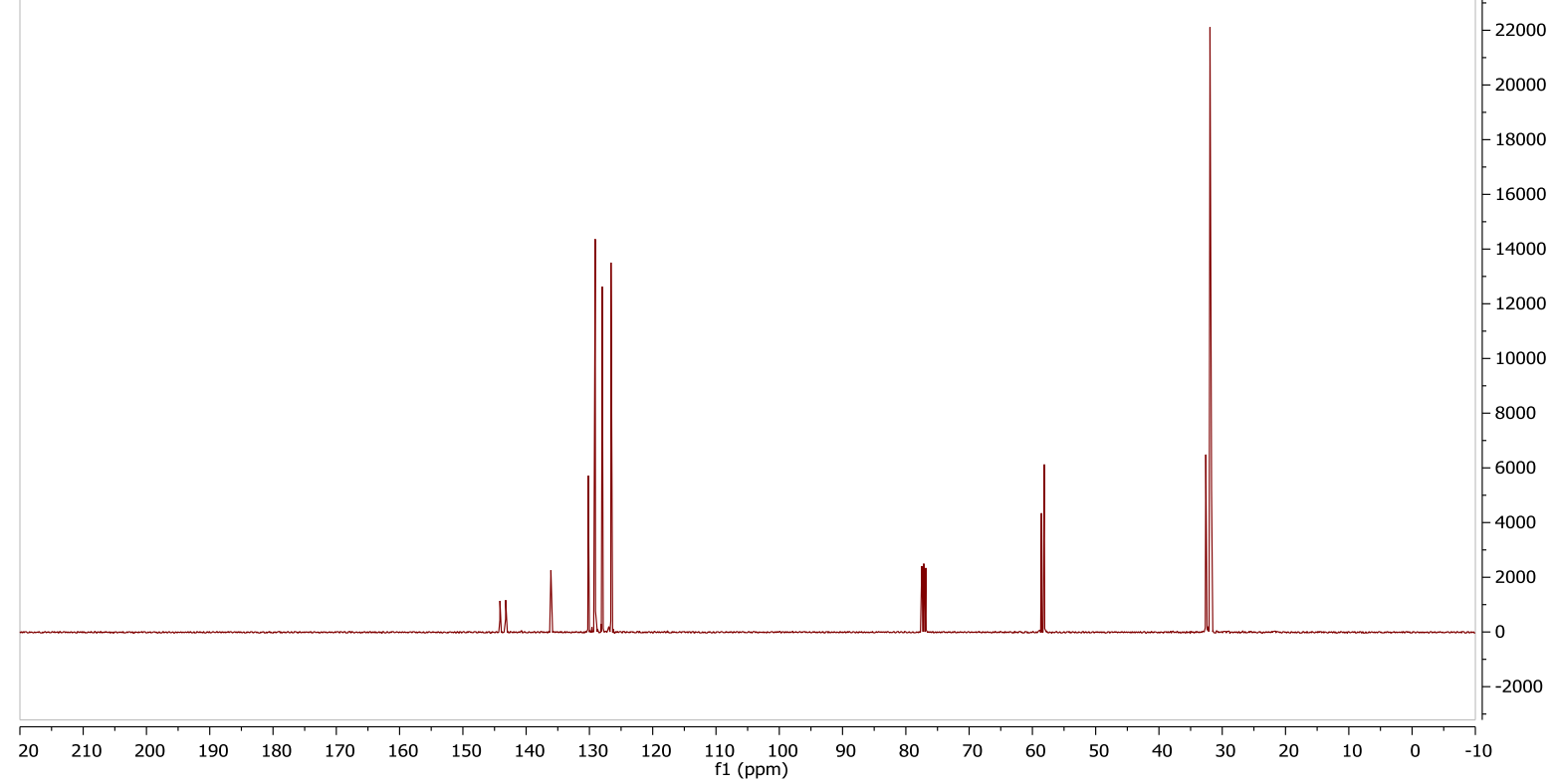



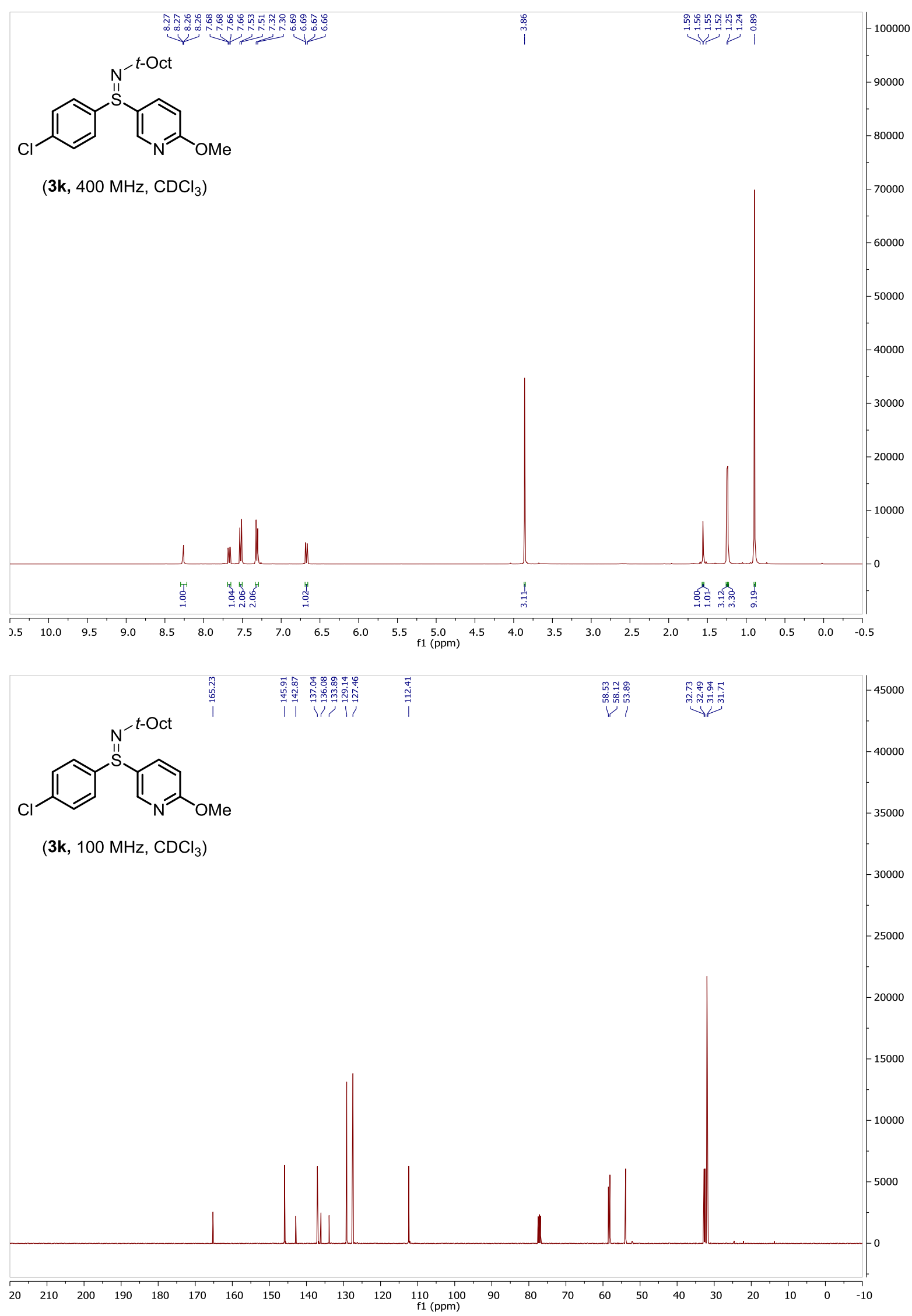

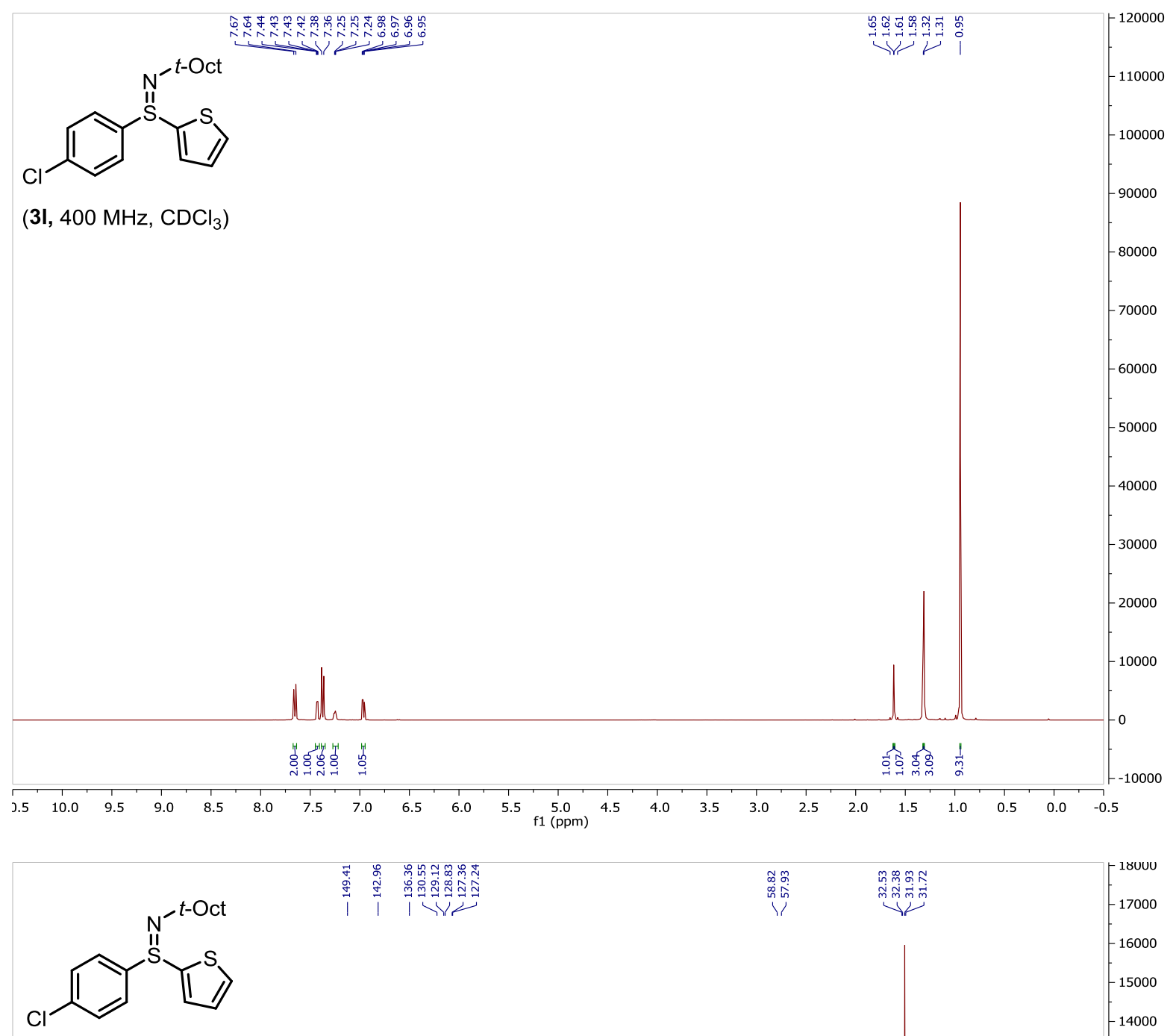

(3I, $100 \mathrm{MHz}, \mathrm{CDCl}_{3}$ )

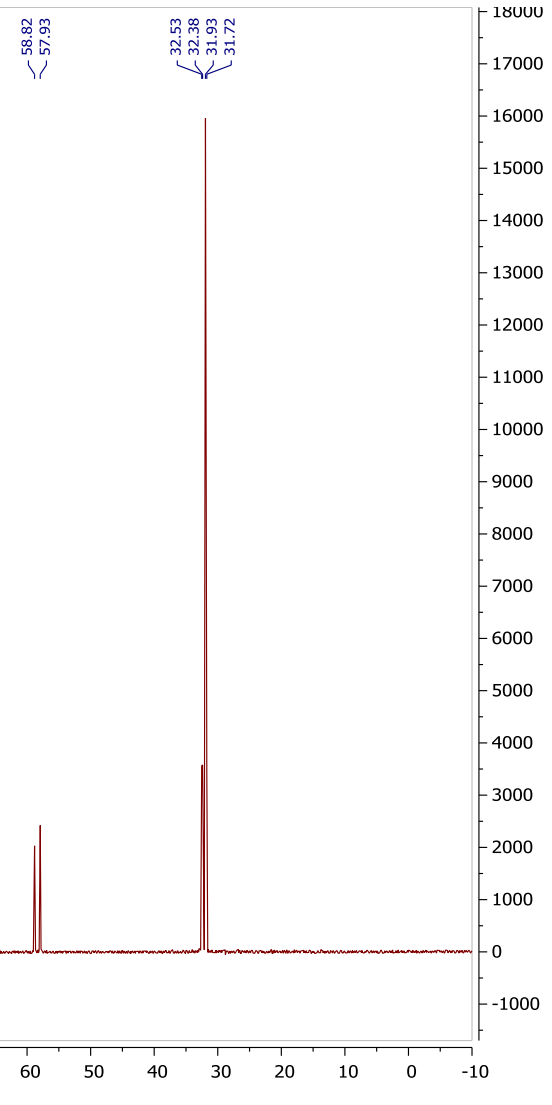



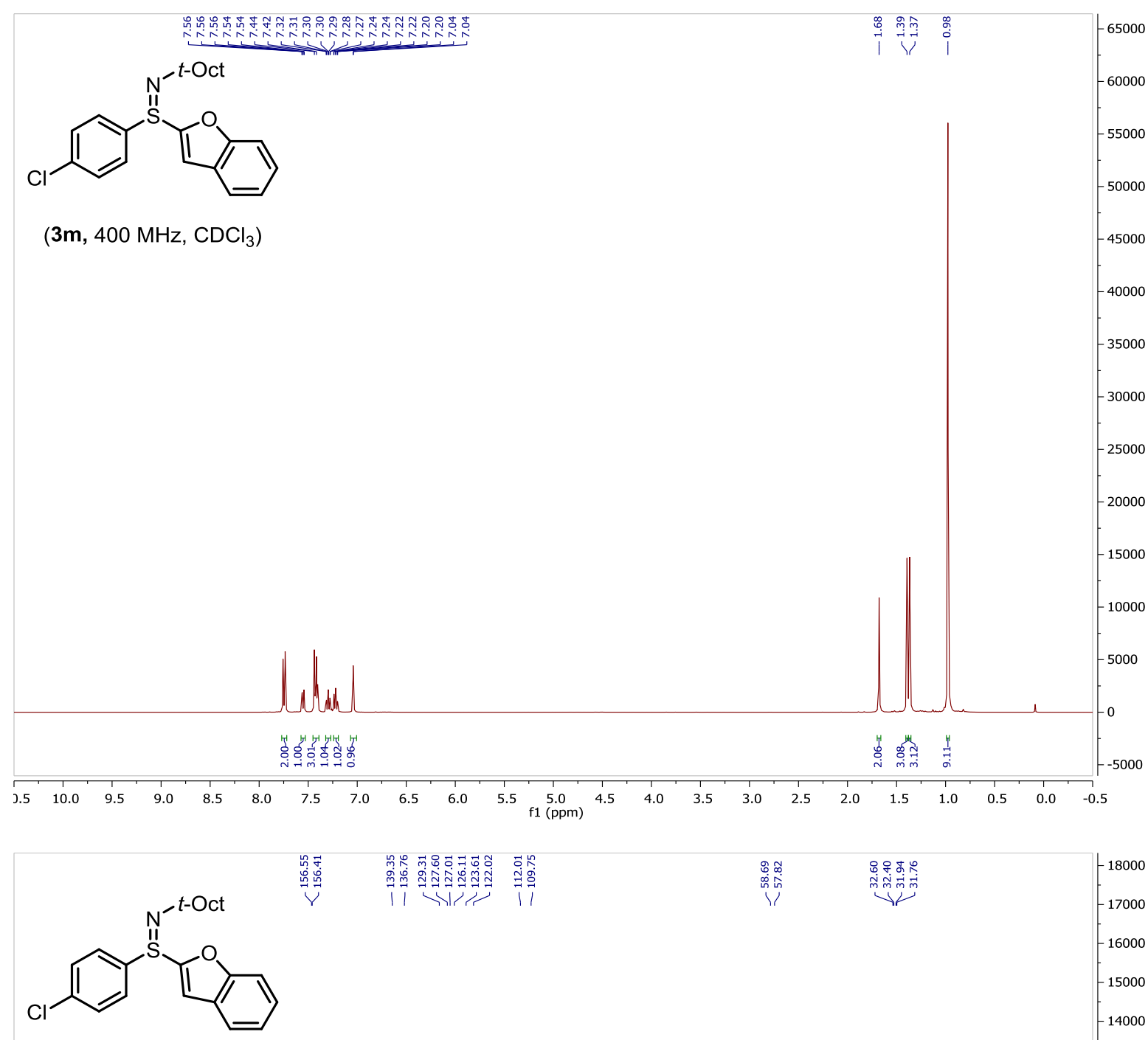

(3m, $100 \mathrm{MHz}, \mathrm{CDCl}_{3}$ )

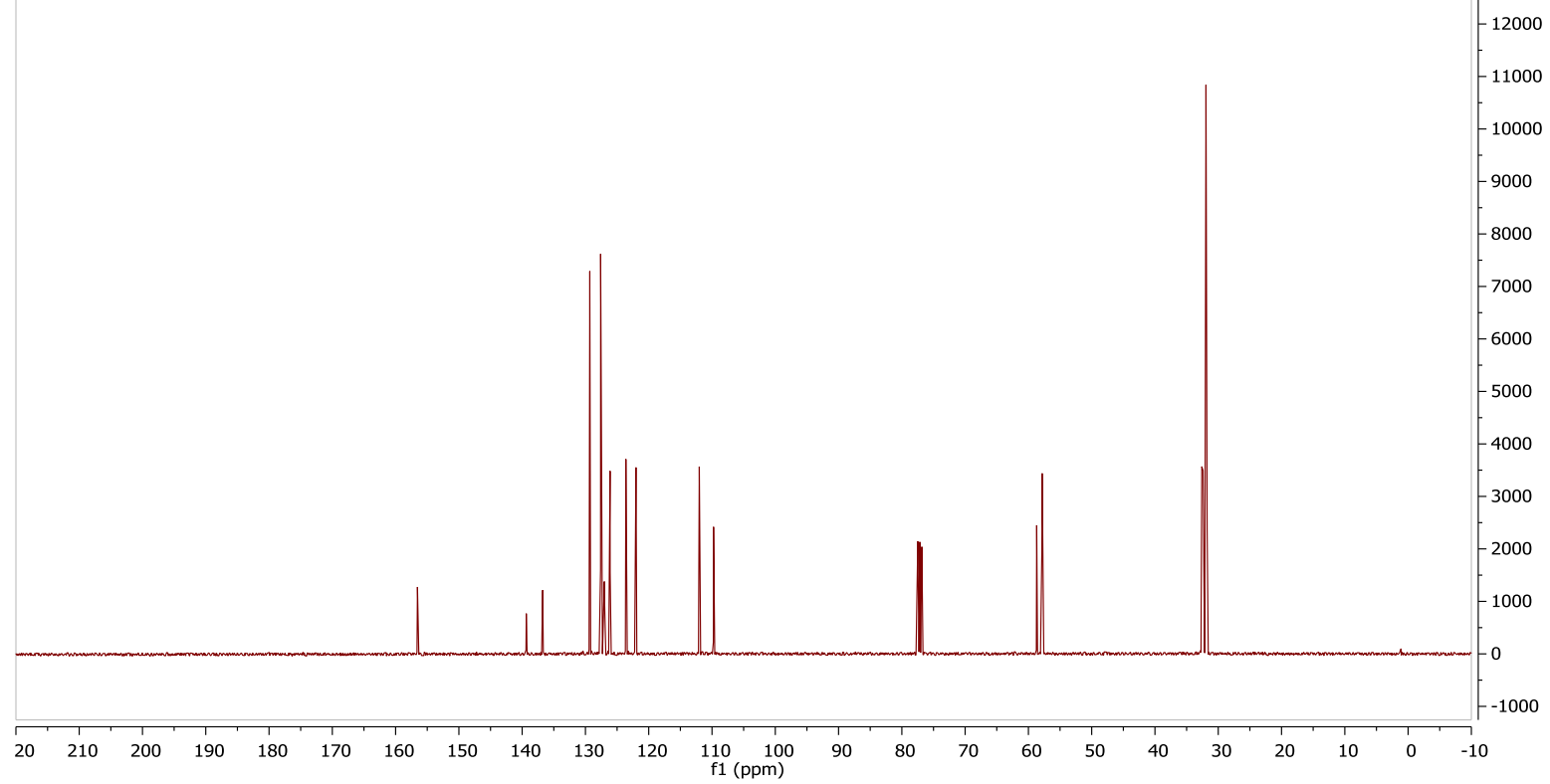




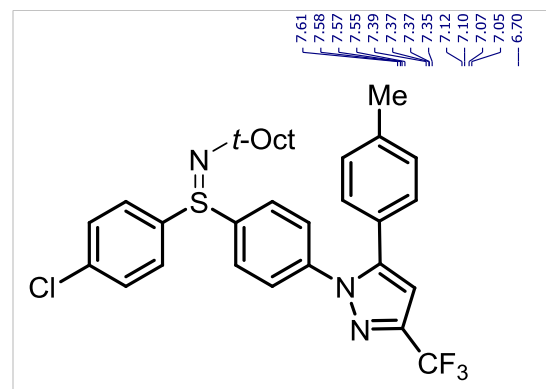

(3n, $400 \mathrm{MHz}, \mathrm{CDCl}_{3}$ )
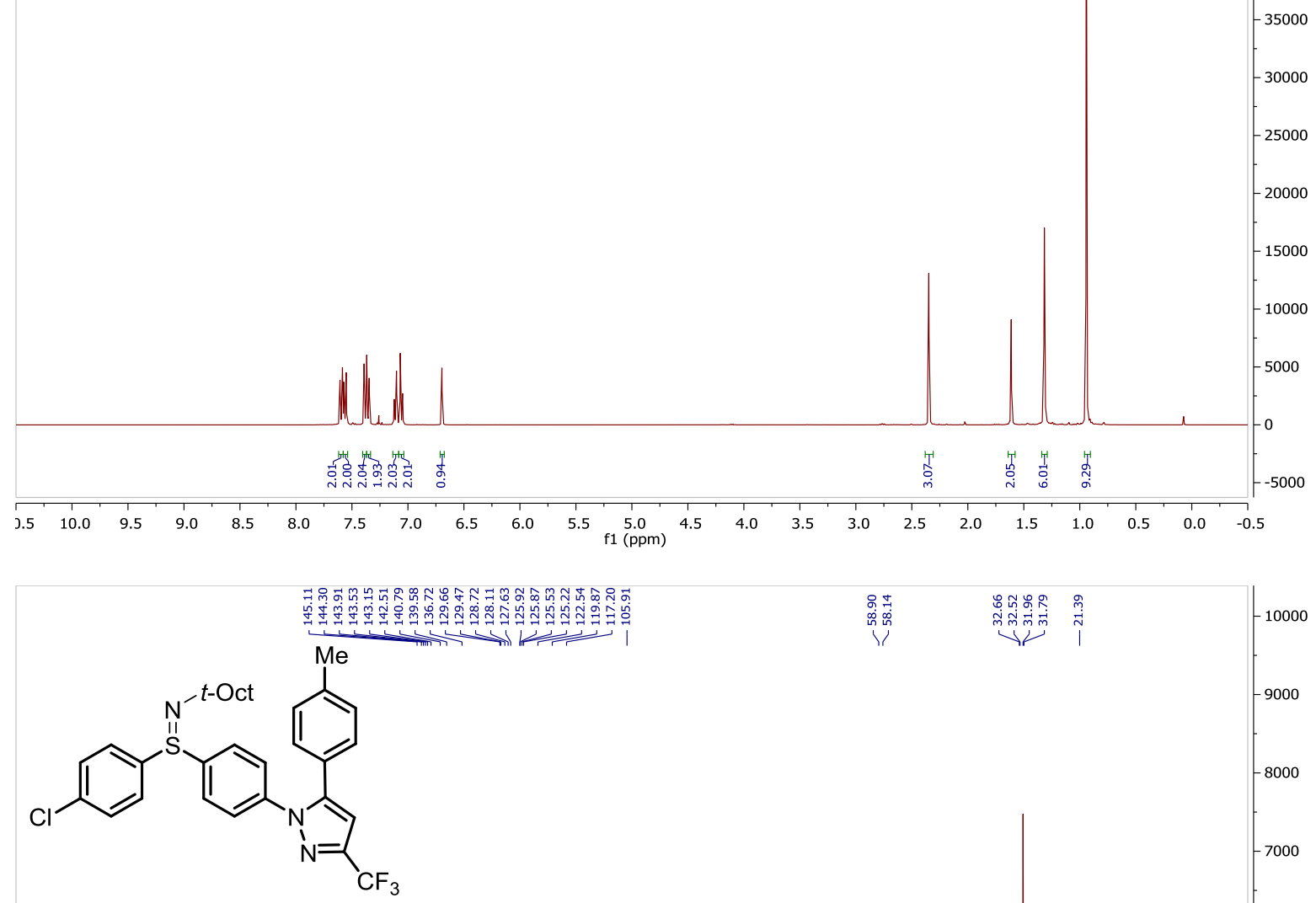

(3n, $100 \mathrm{MHz}, \mathrm{CDCl}_{3}$ )
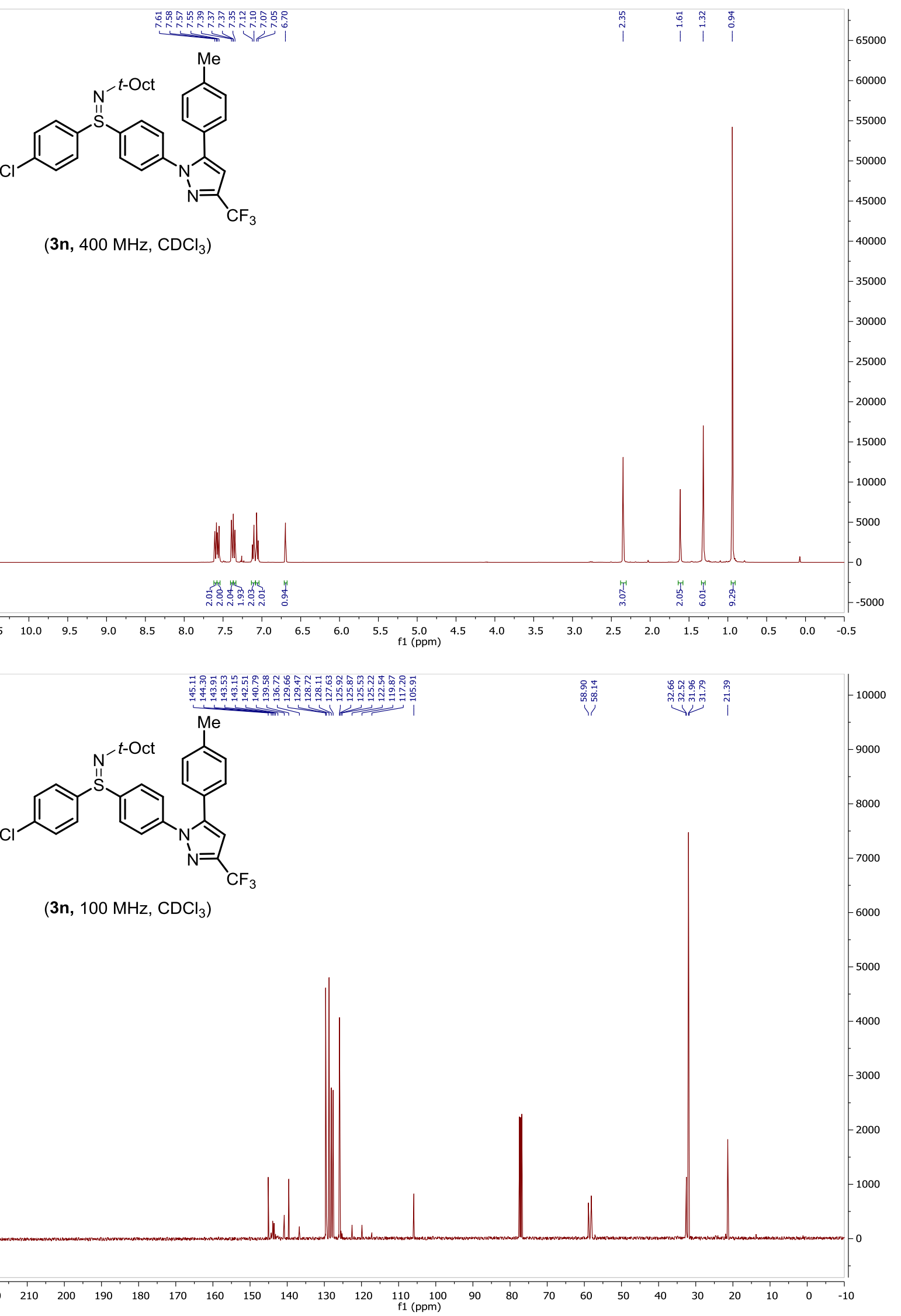


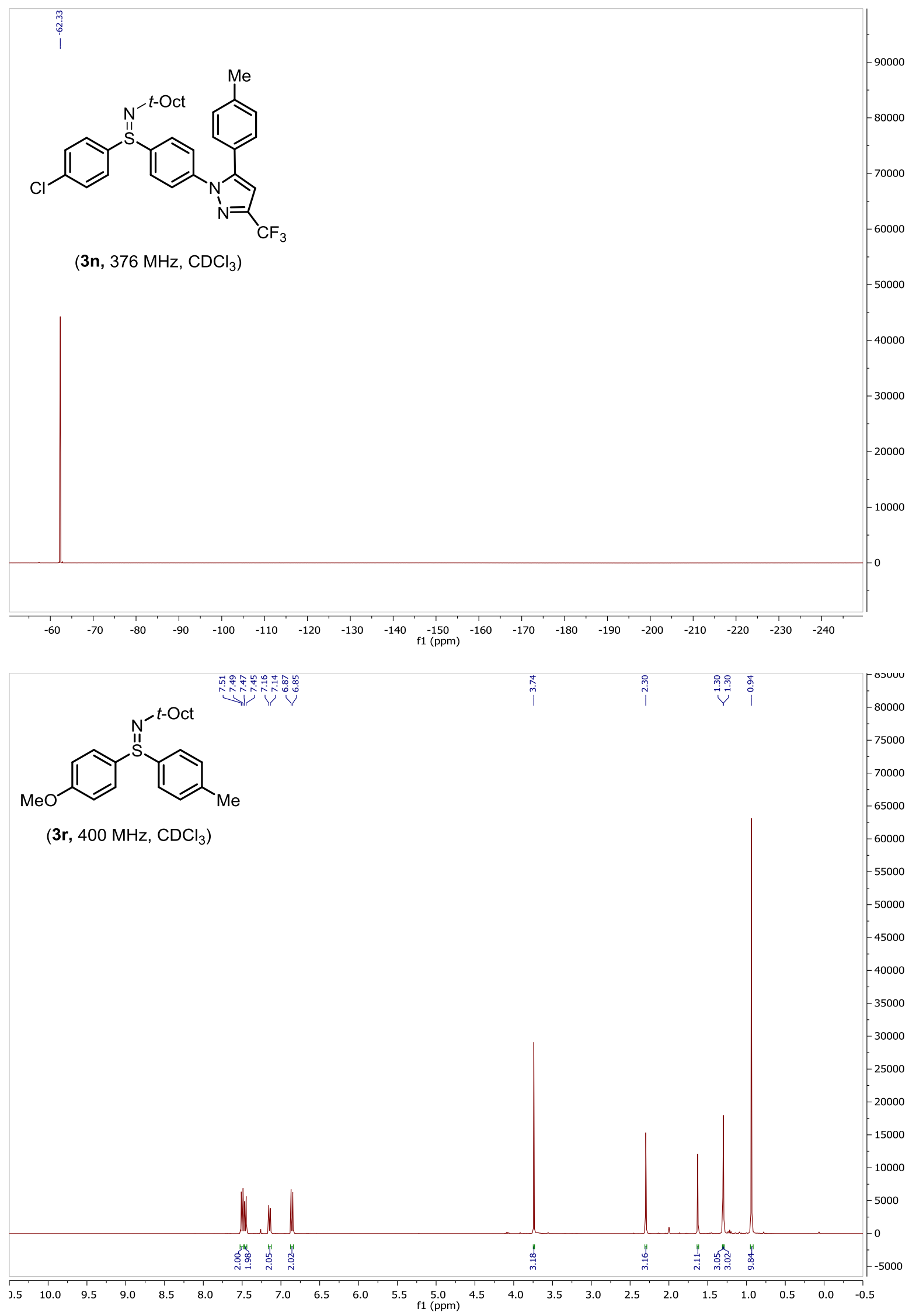



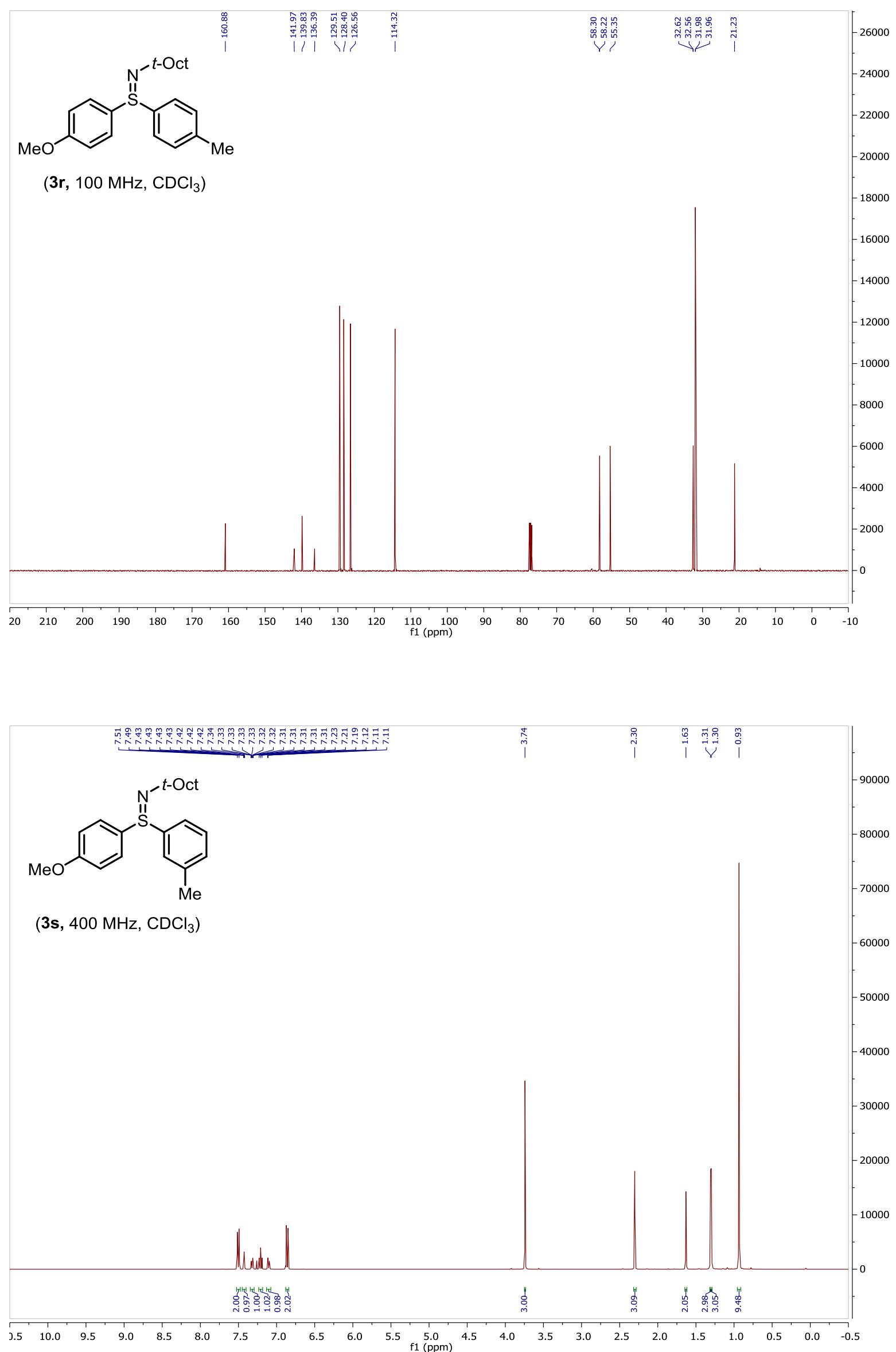

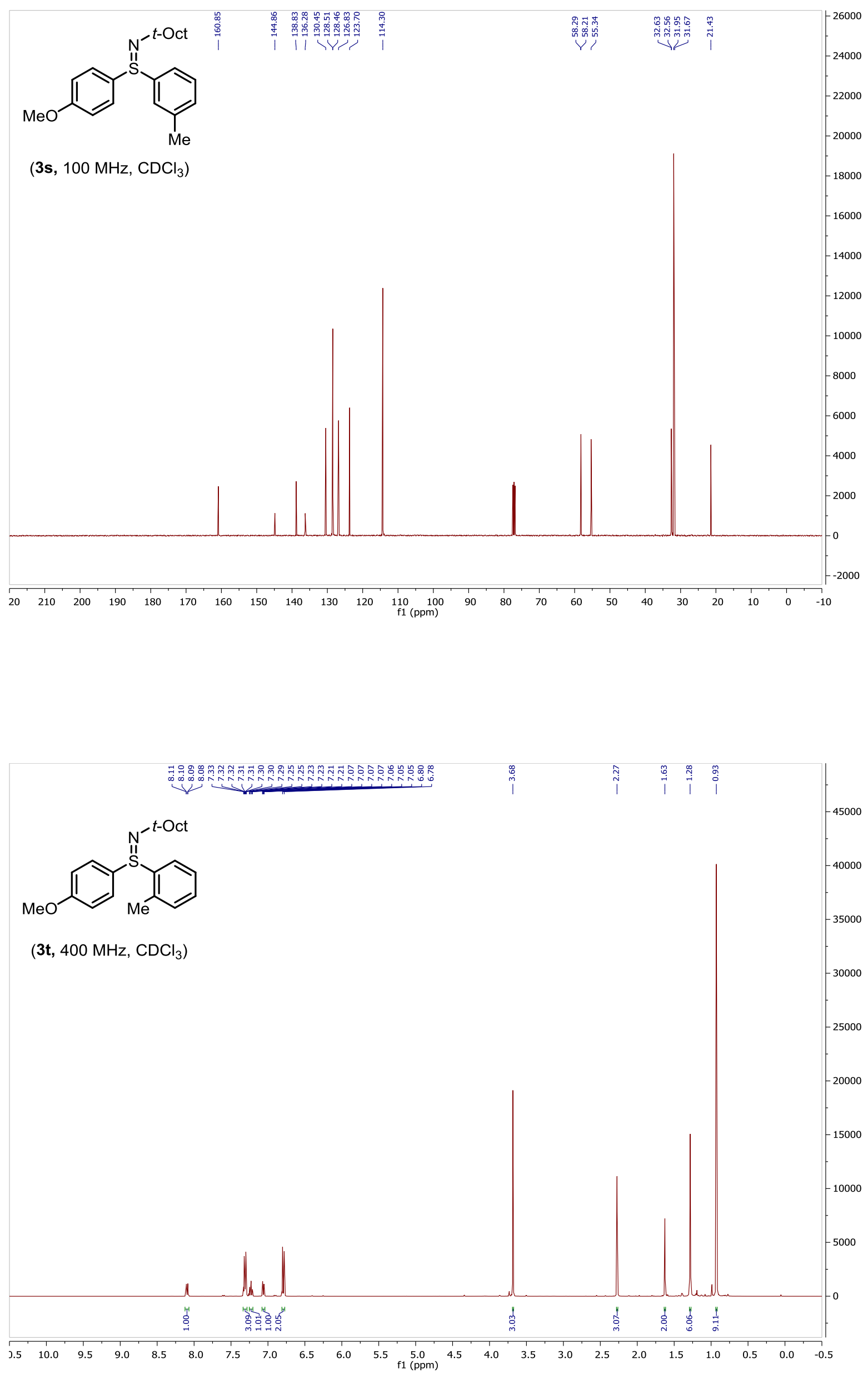

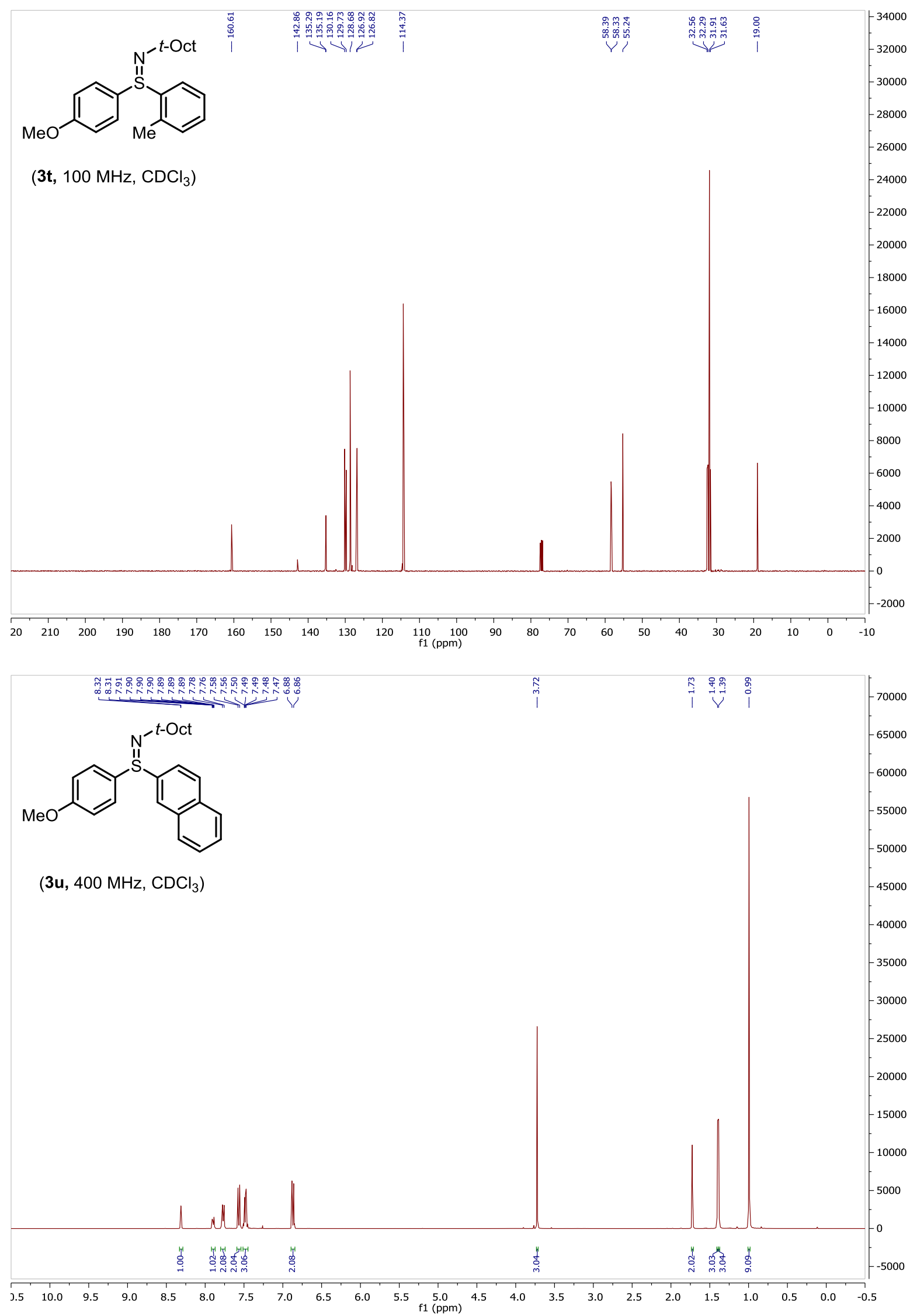

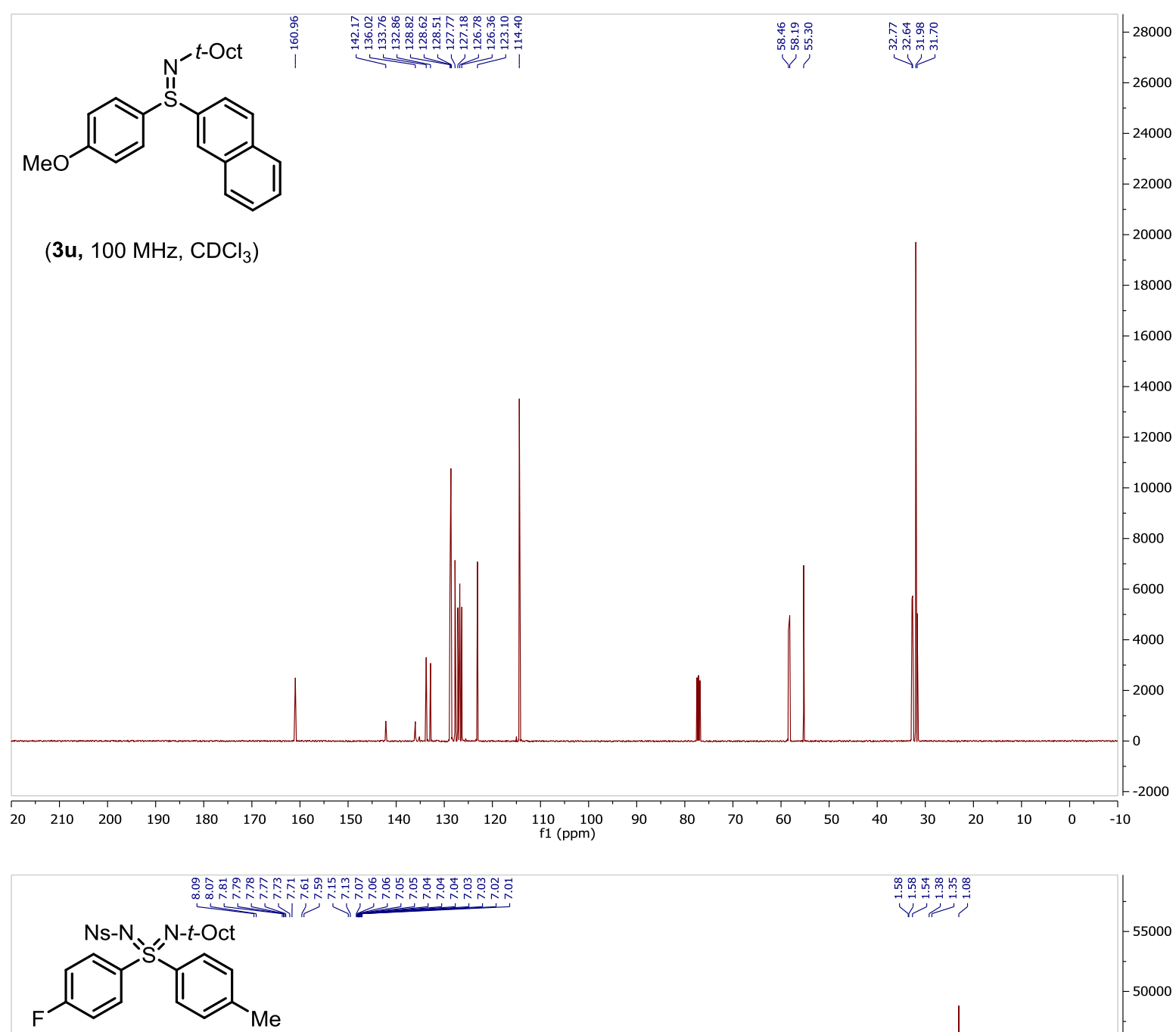

(4a, $400 \mathrm{MHz}, \mathrm{CDCl}_{3}$ )

\section{تص象}

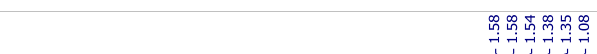



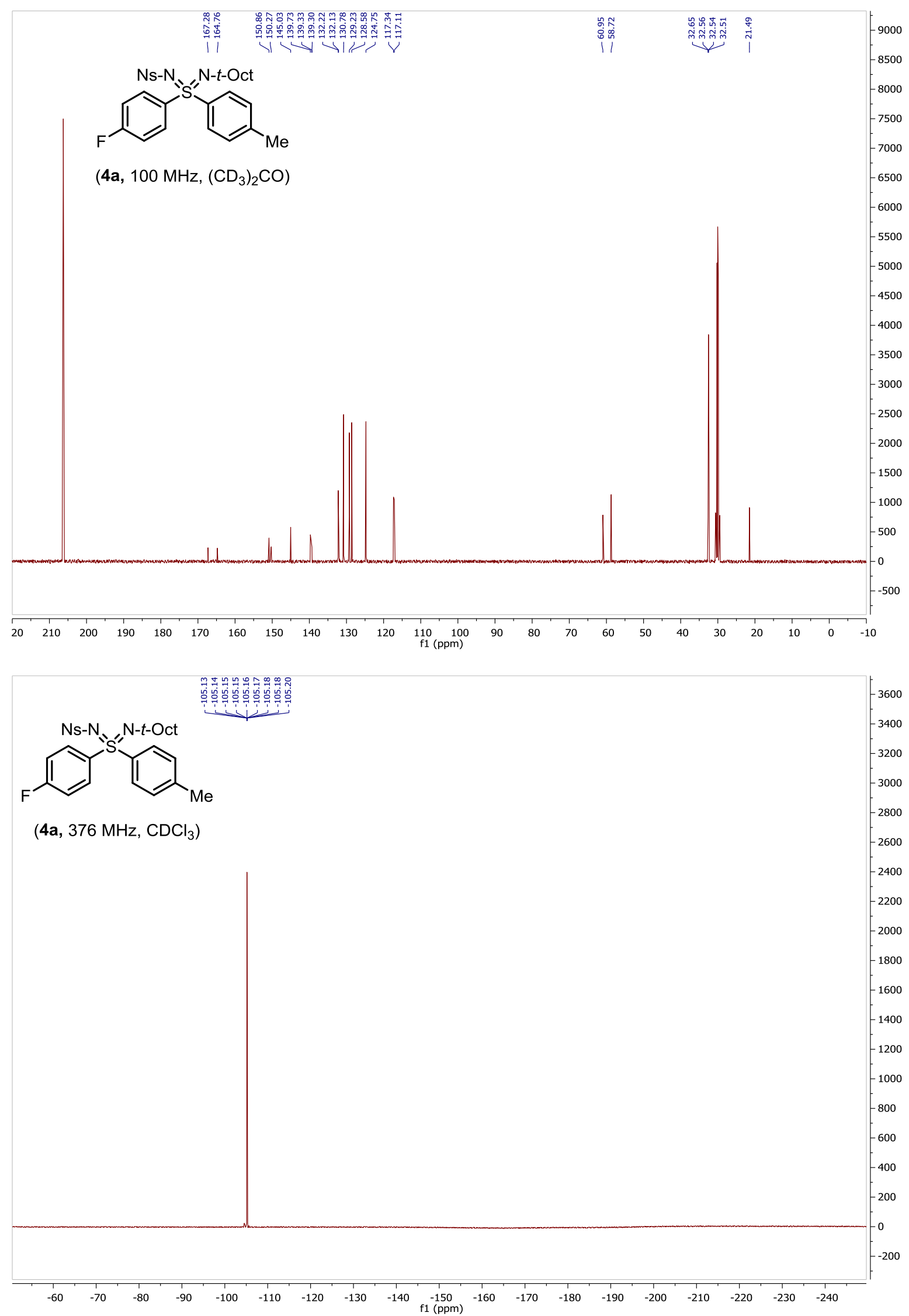


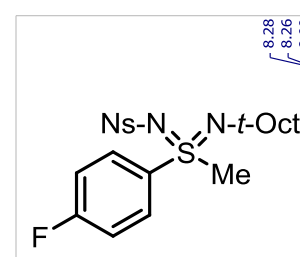

(4b, $400 \mathrm{MHz}, \mathrm{CDCl}_{3}$ )
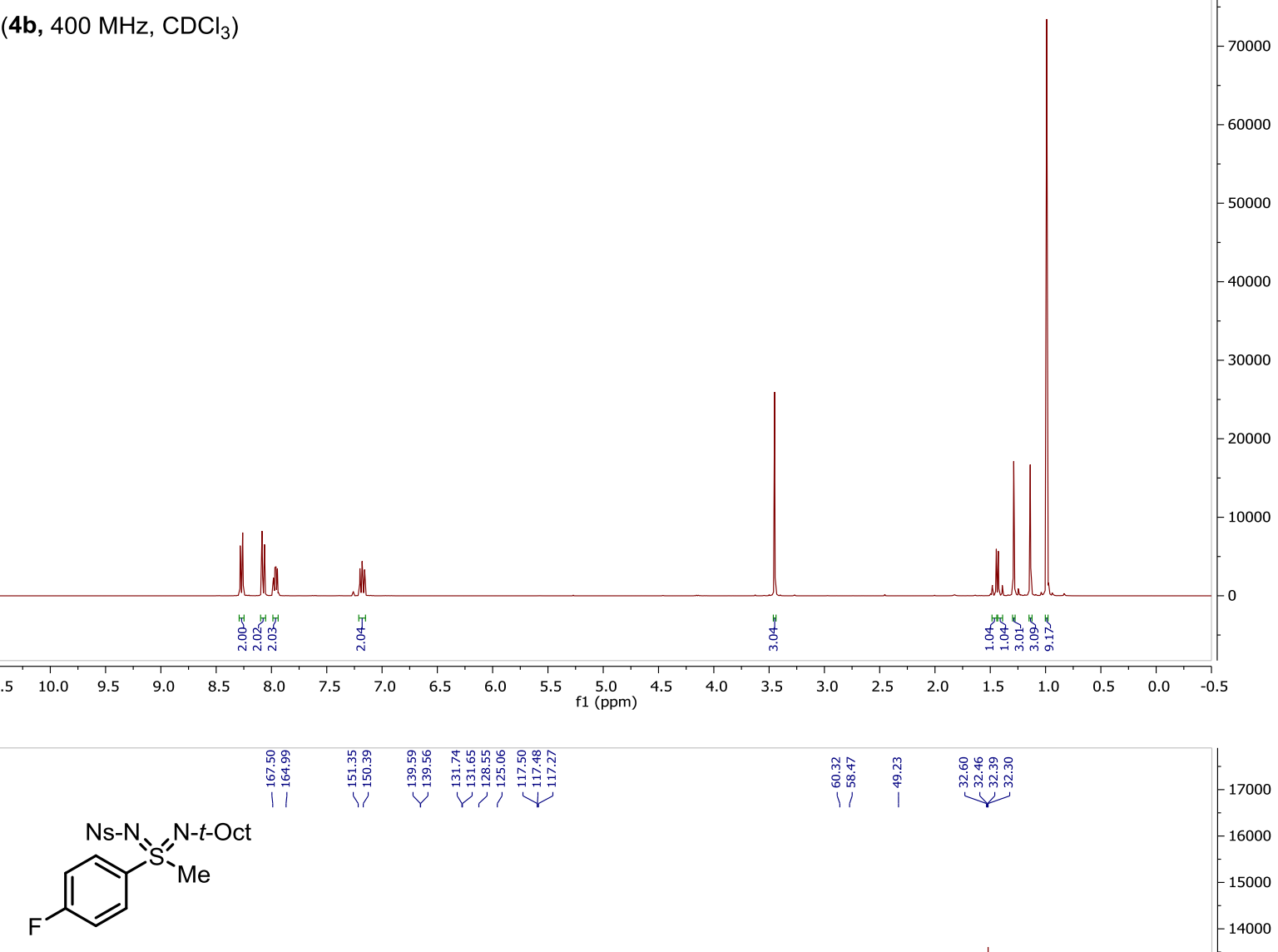

(4b, $\left.100 \mathrm{MHz},\left(\mathrm{CD}_{3}\right)_{2} \mathrm{CO}\right)$

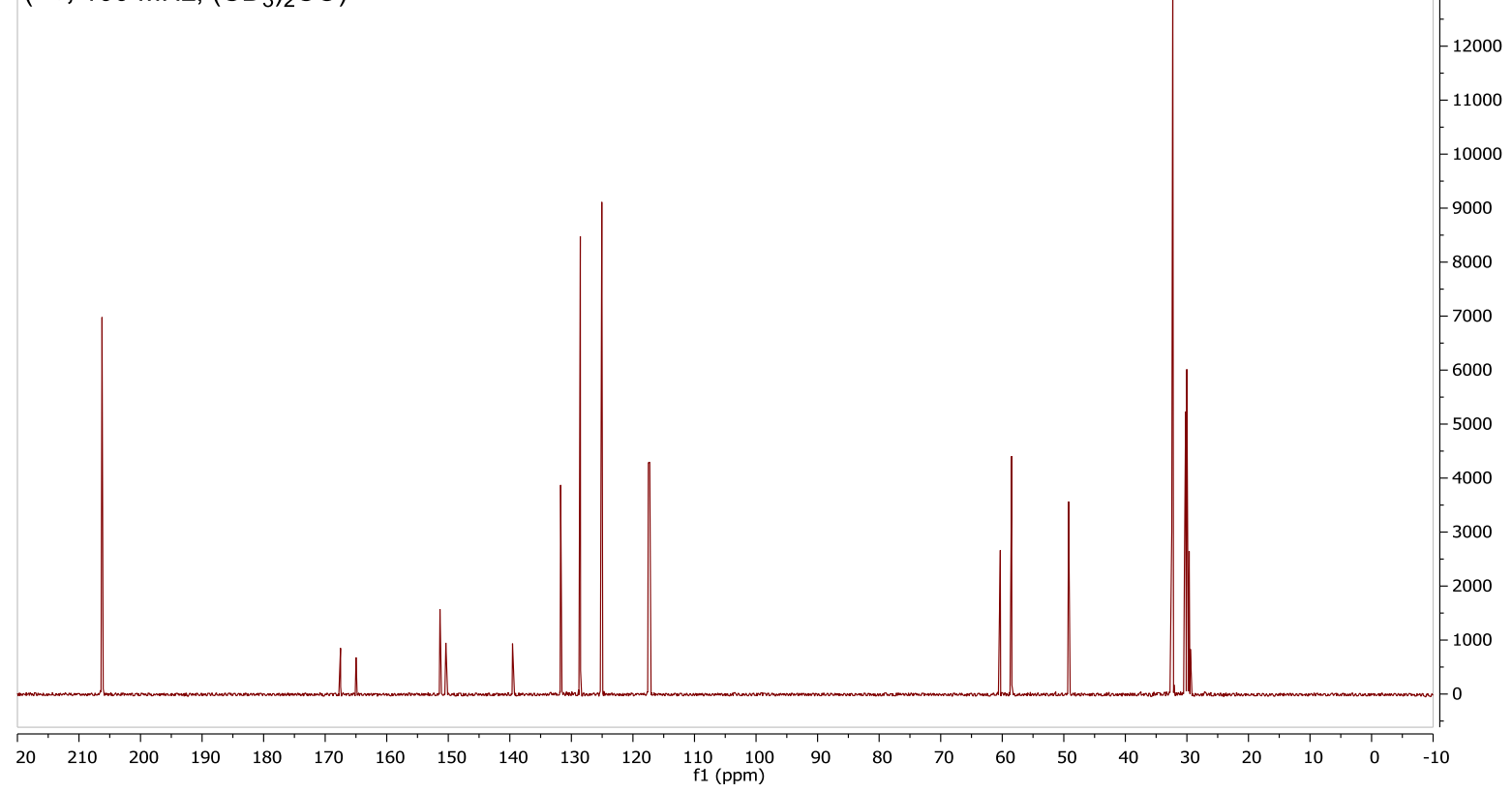




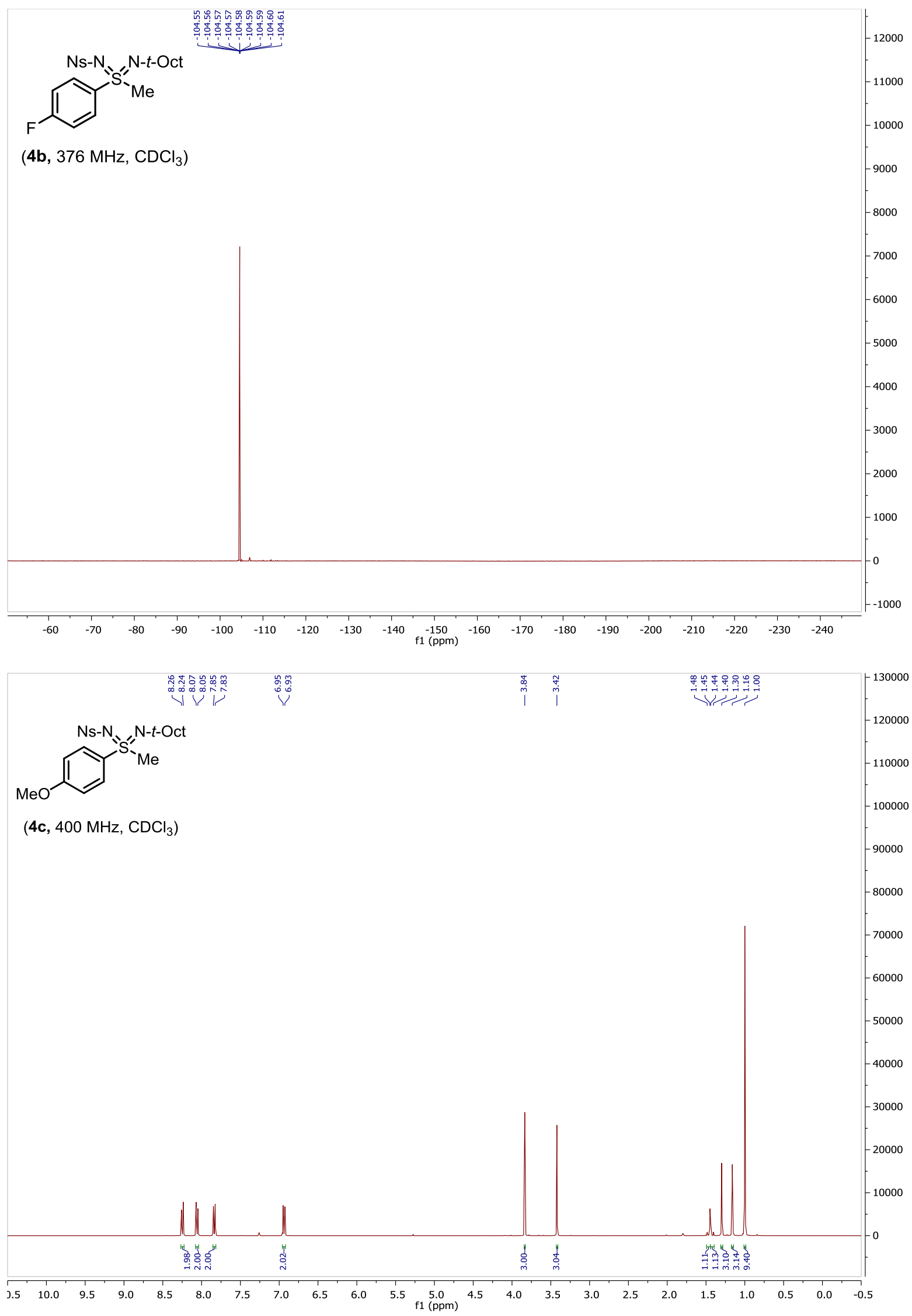




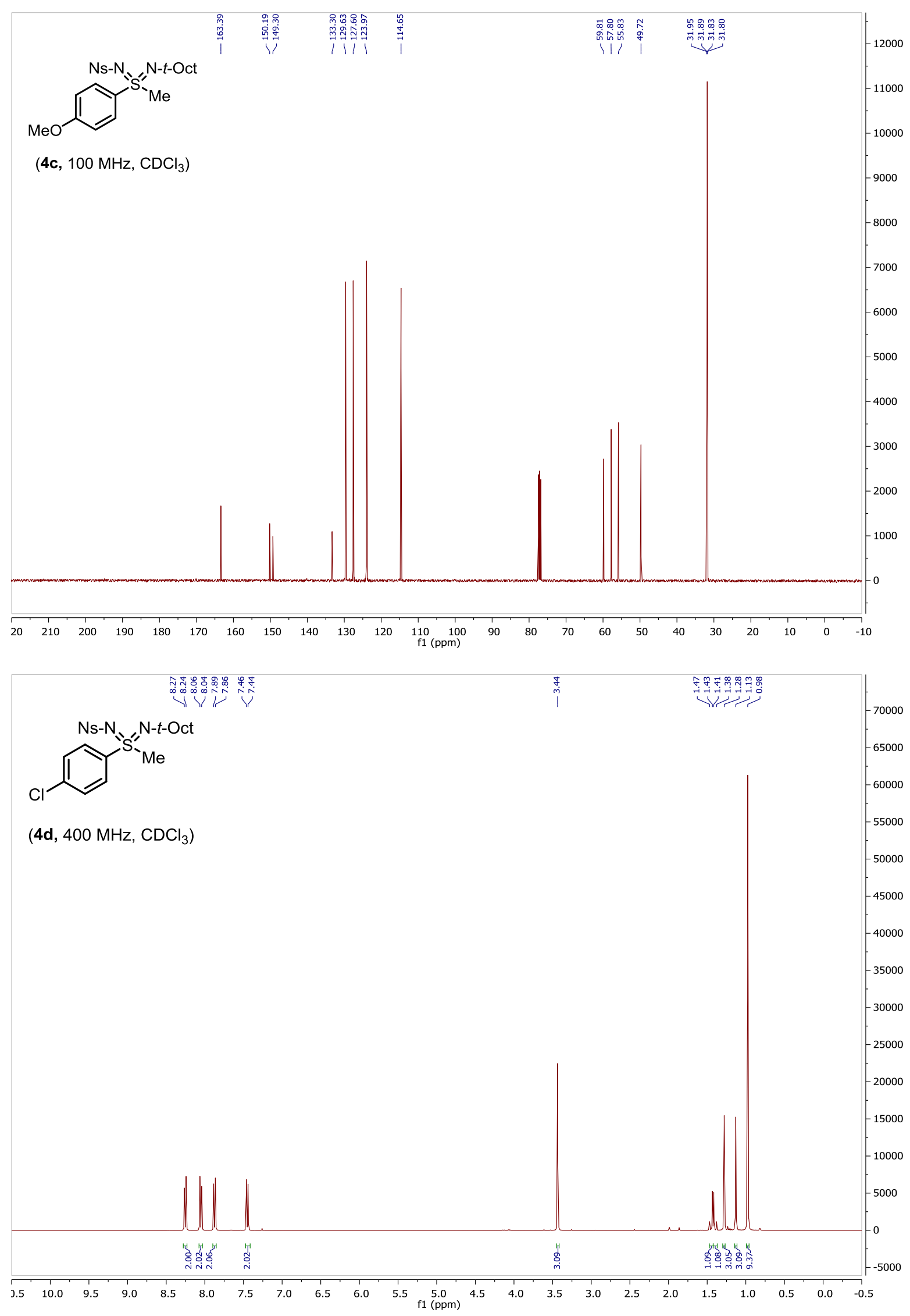




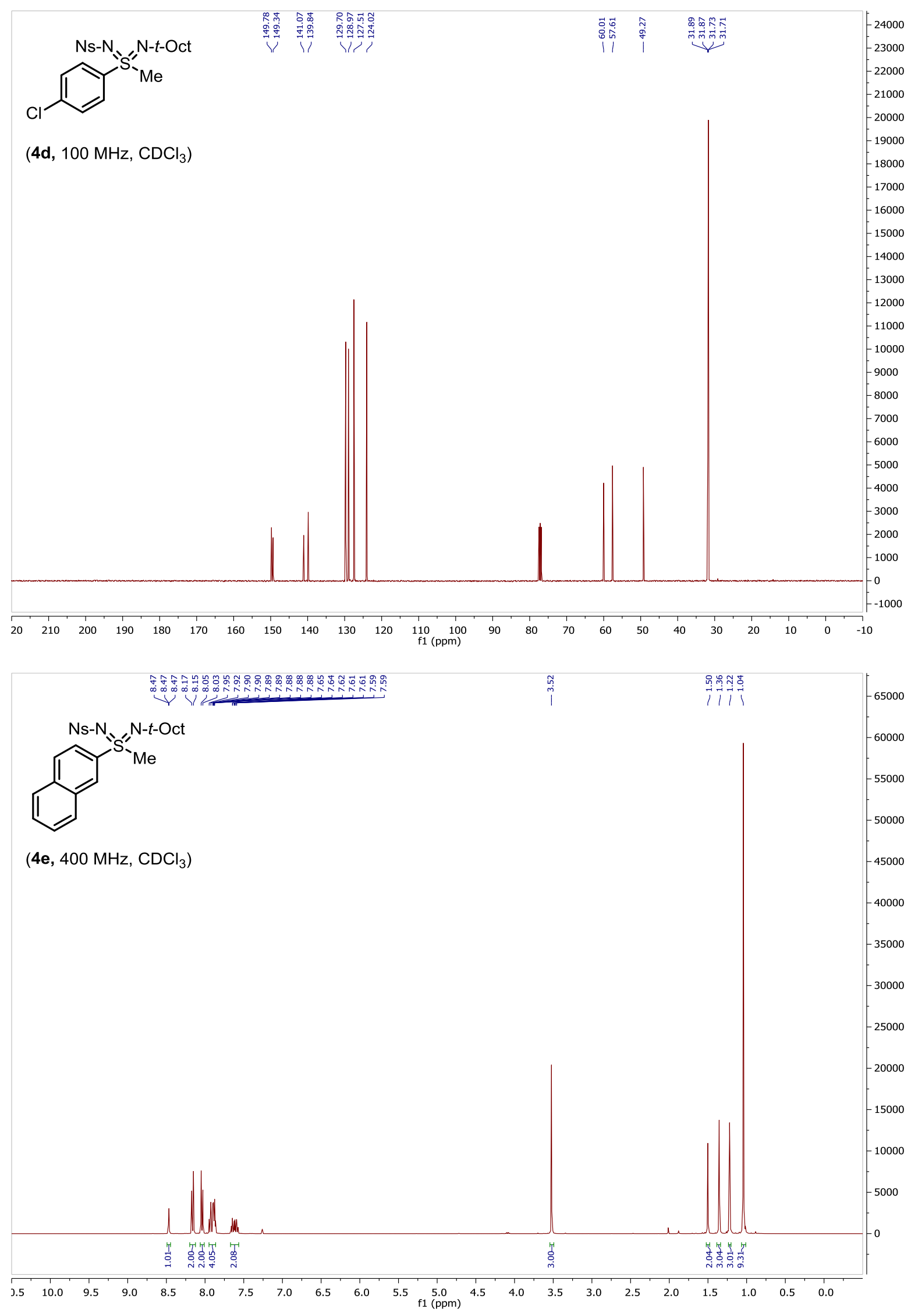




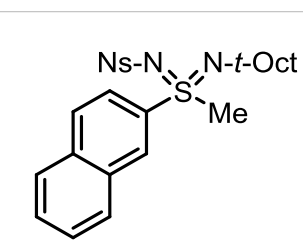

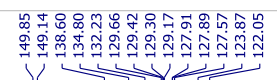

(4e, $100 \mathrm{MHz}, \mathrm{CDCl}_{3}$ )
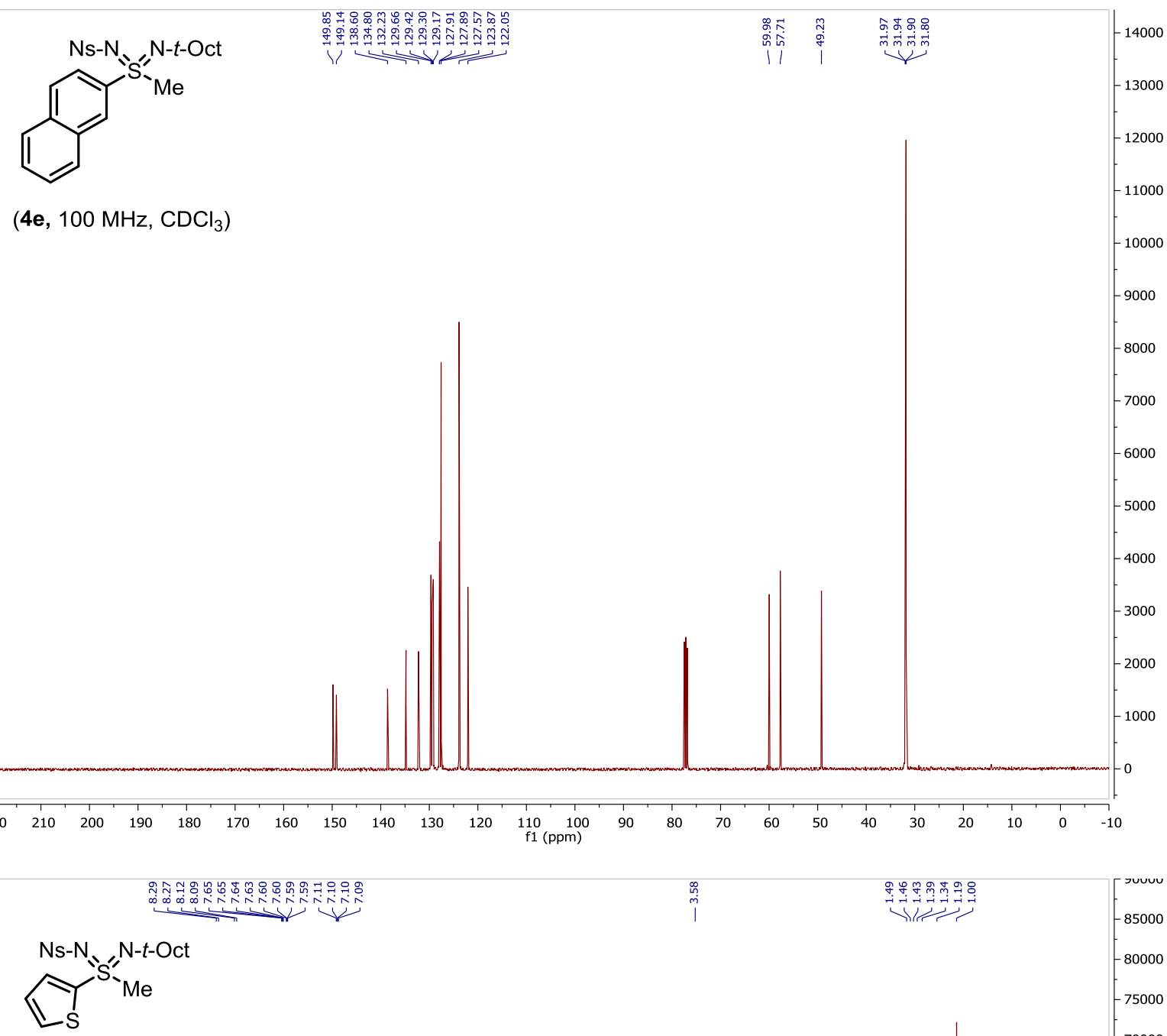

(4f, $400 \mathrm{MHz}, \mathrm{CDCl}_{3}$ )

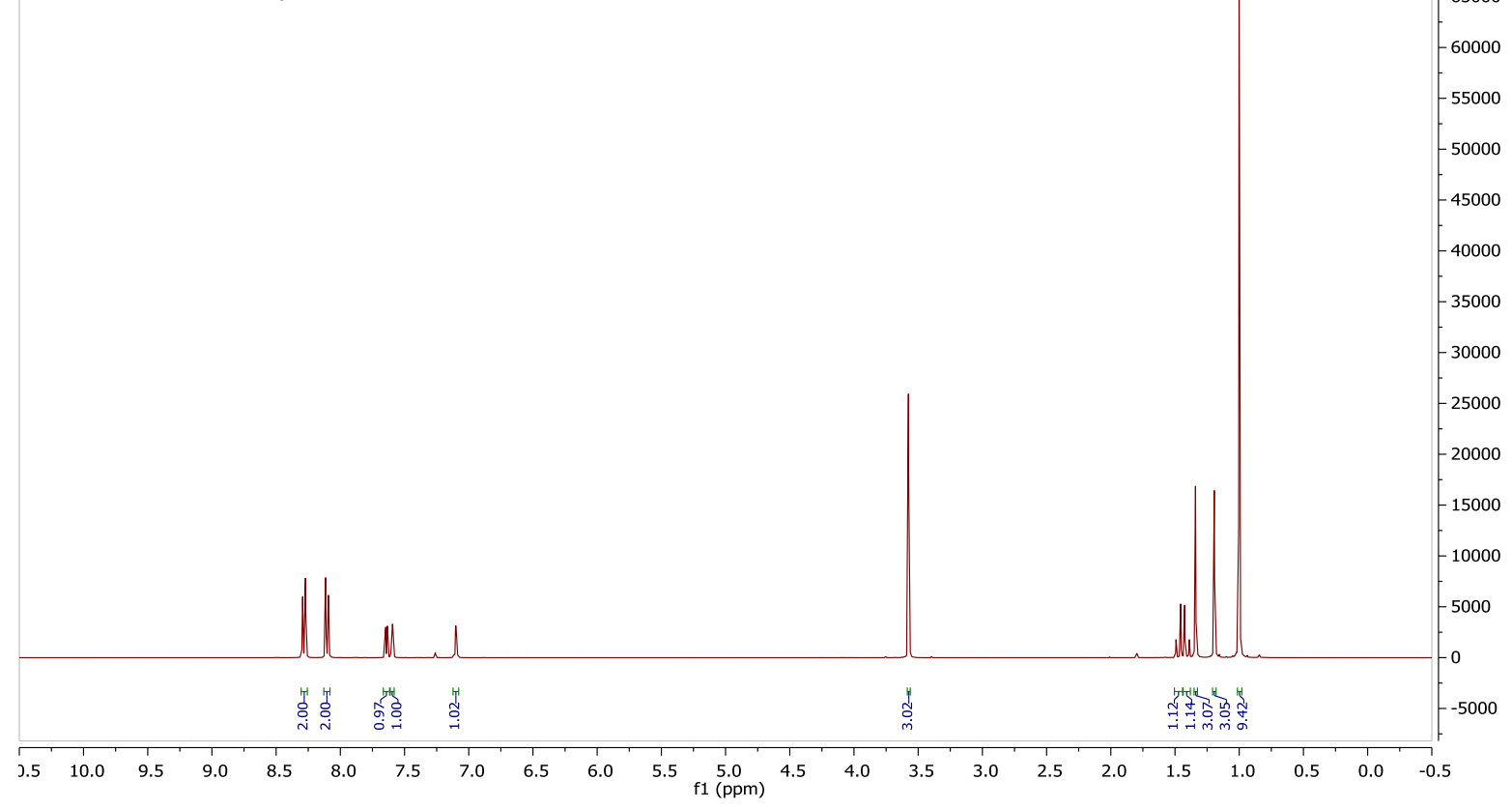




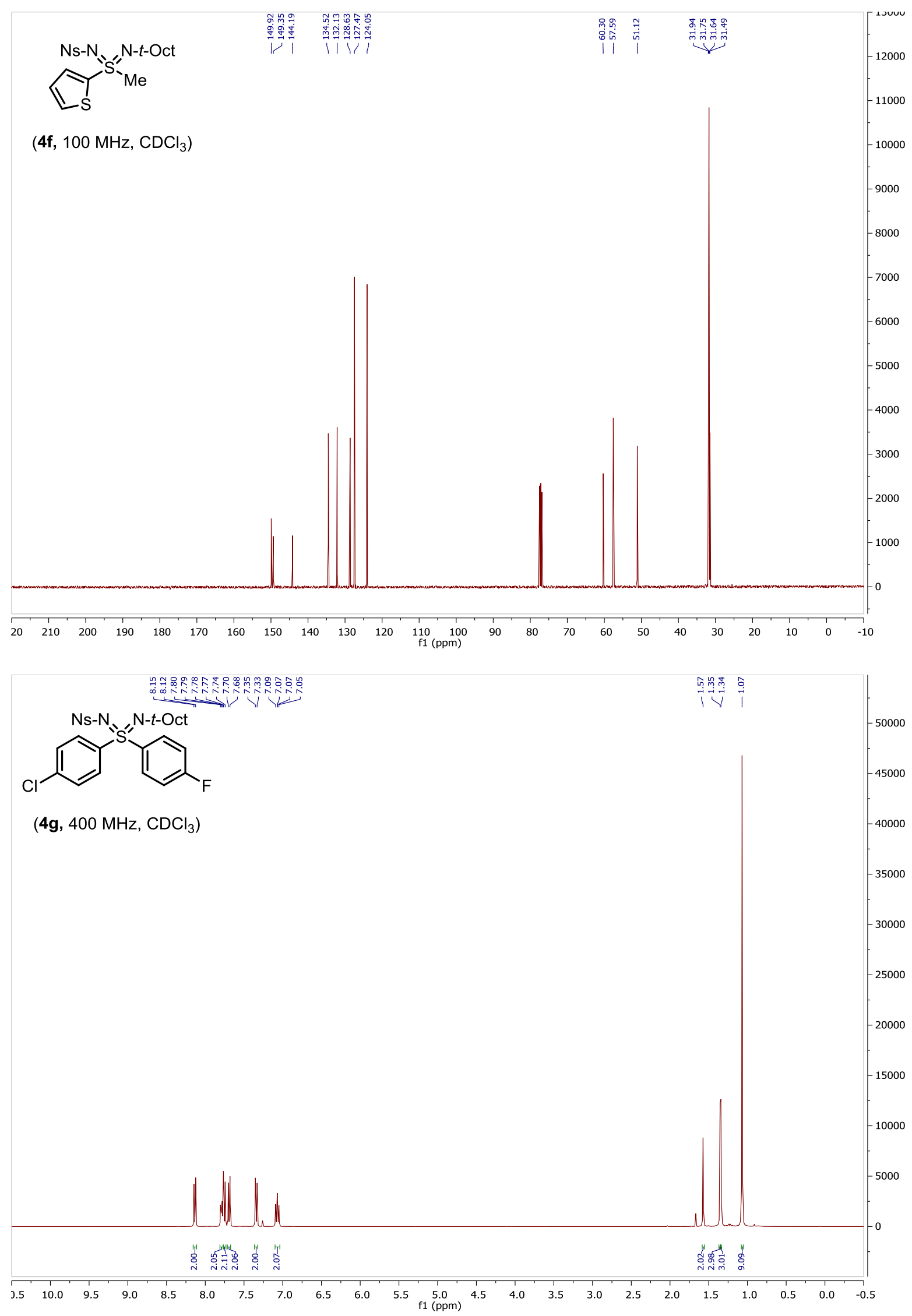



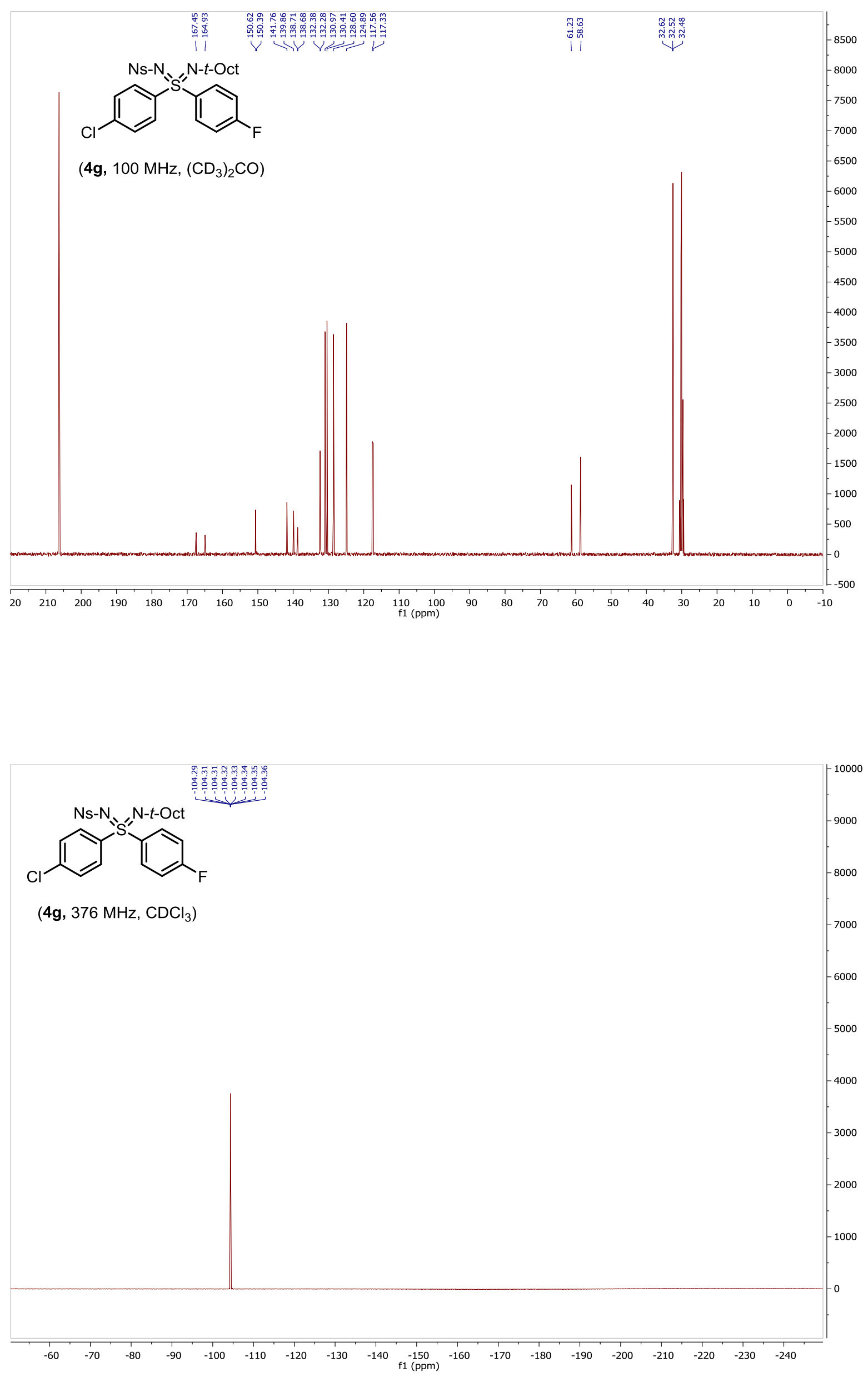

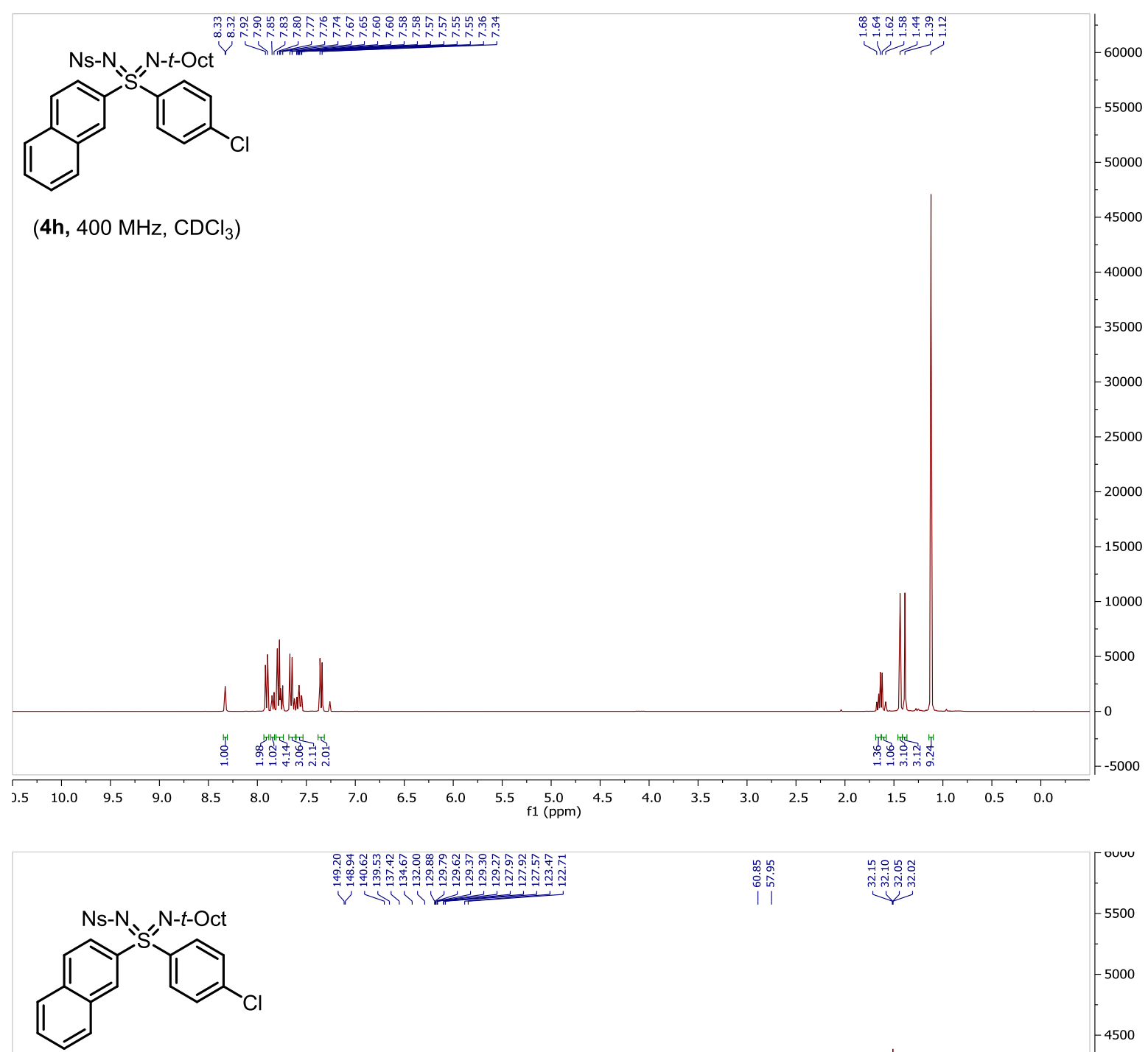

(4h, $100 \mathrm{MHz}, \mathrm{CDCl}_{3}$ )
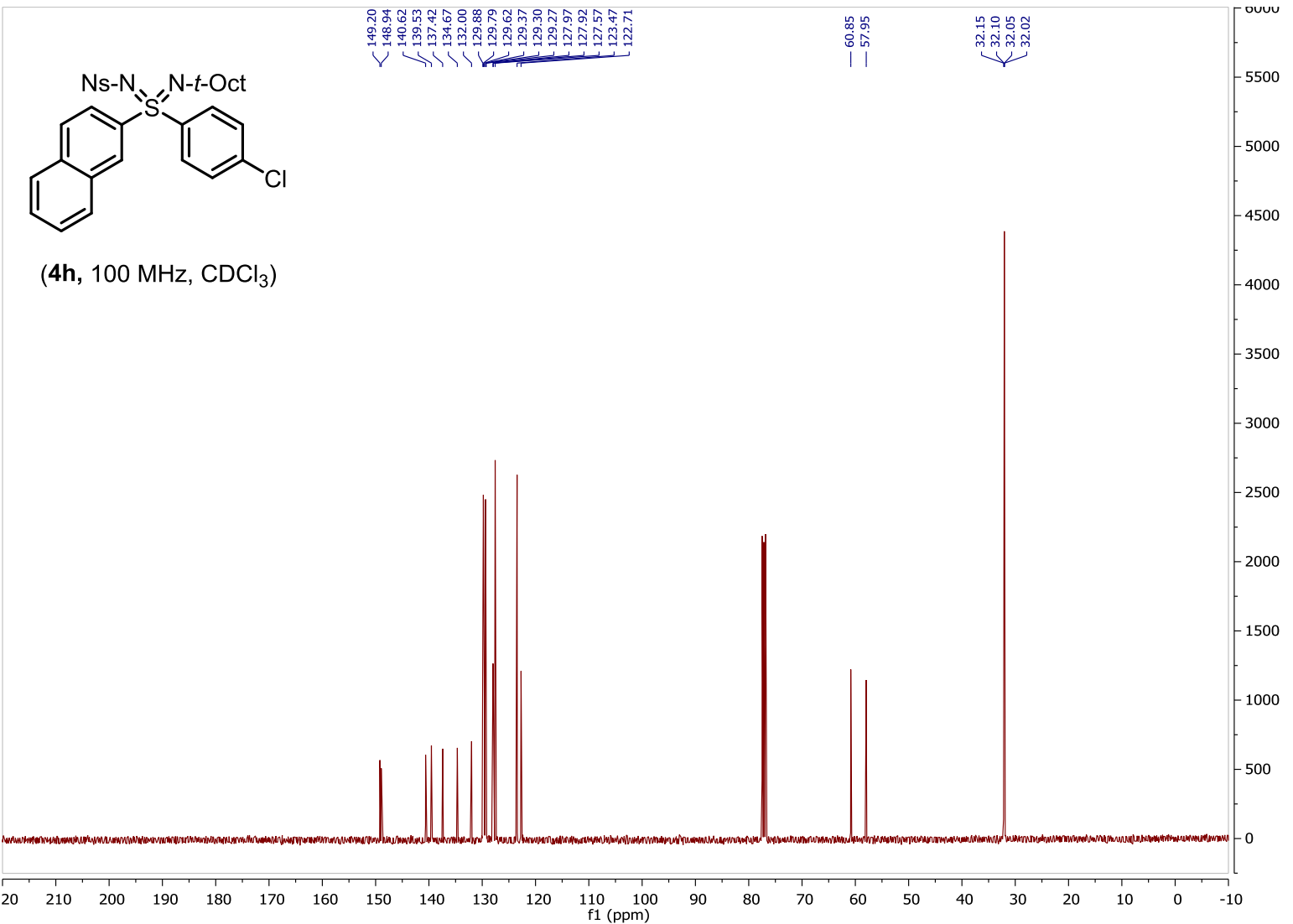

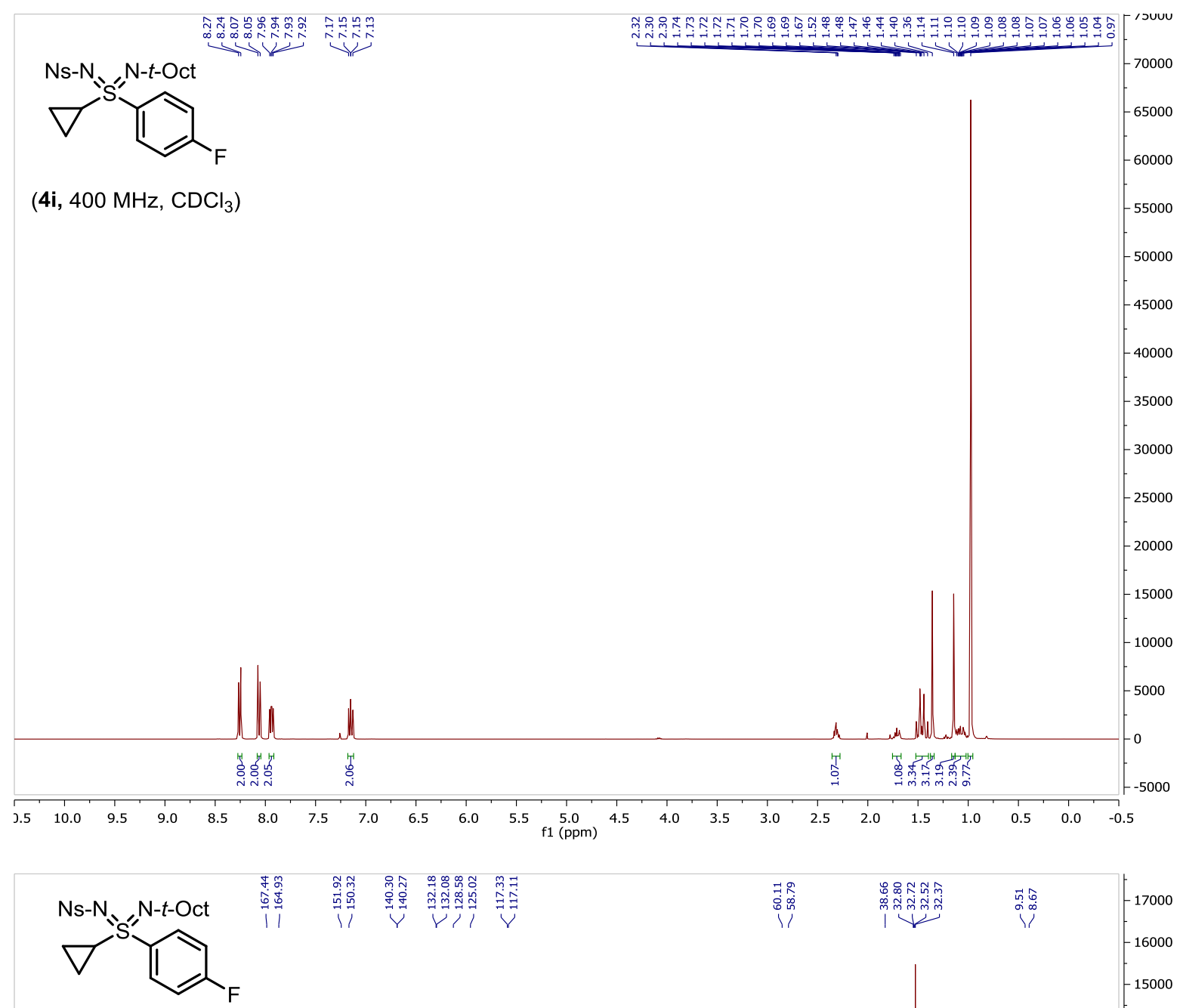

(4i, $\left.100 \mathrm{MHz},\left(\mathrm{CD}_{3}\right)_{2} \mathrm{CO}\right)$

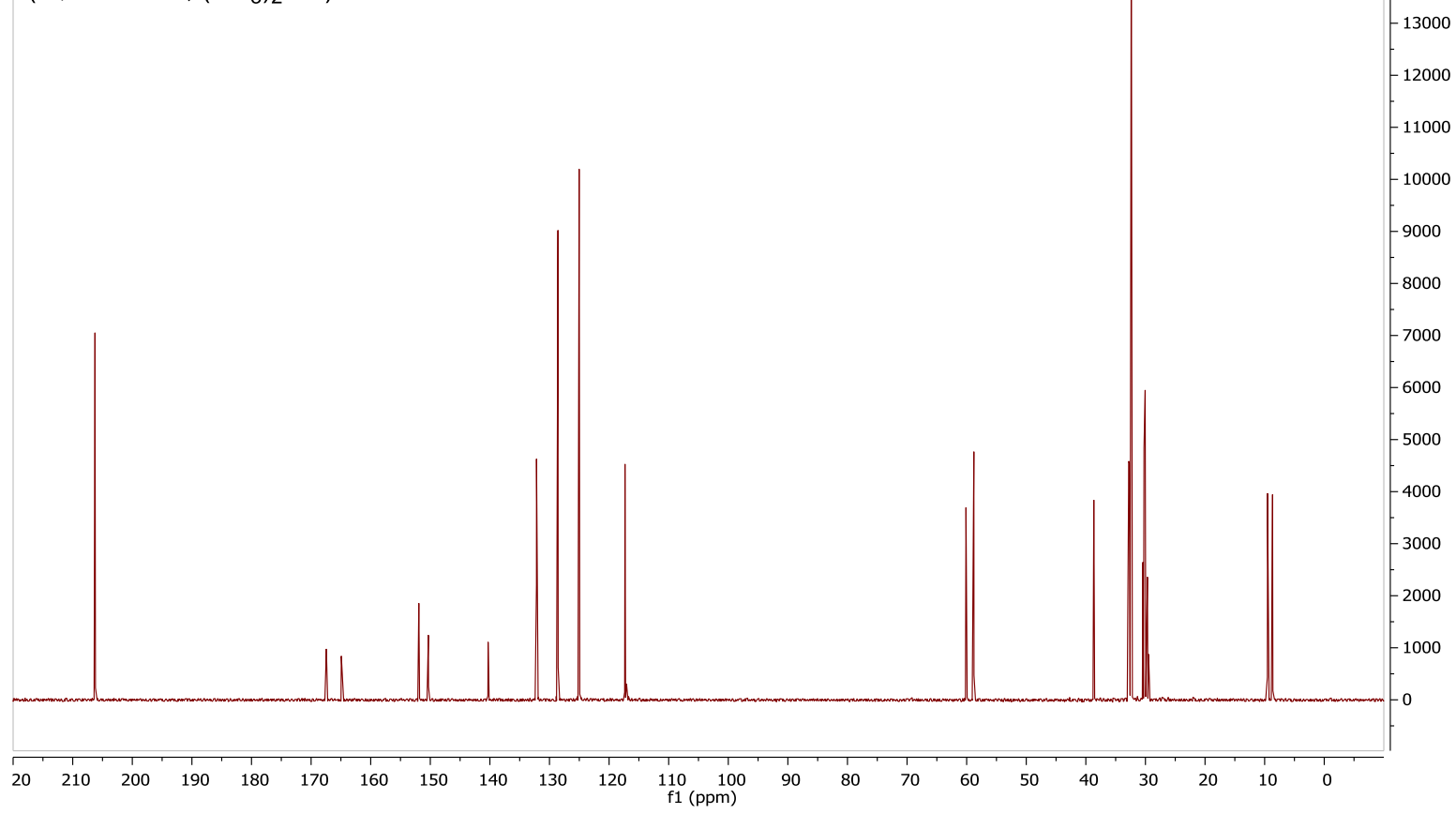



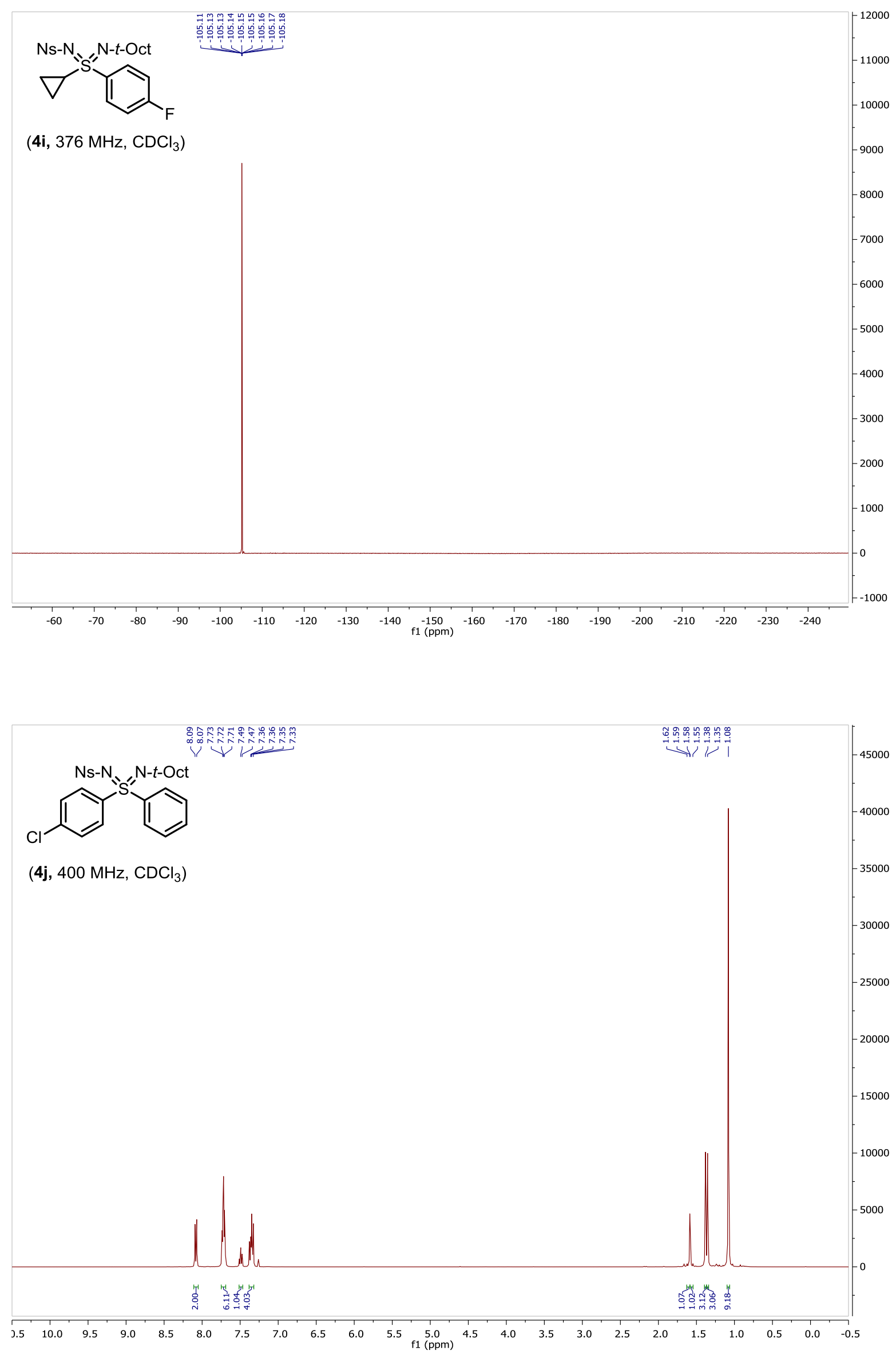


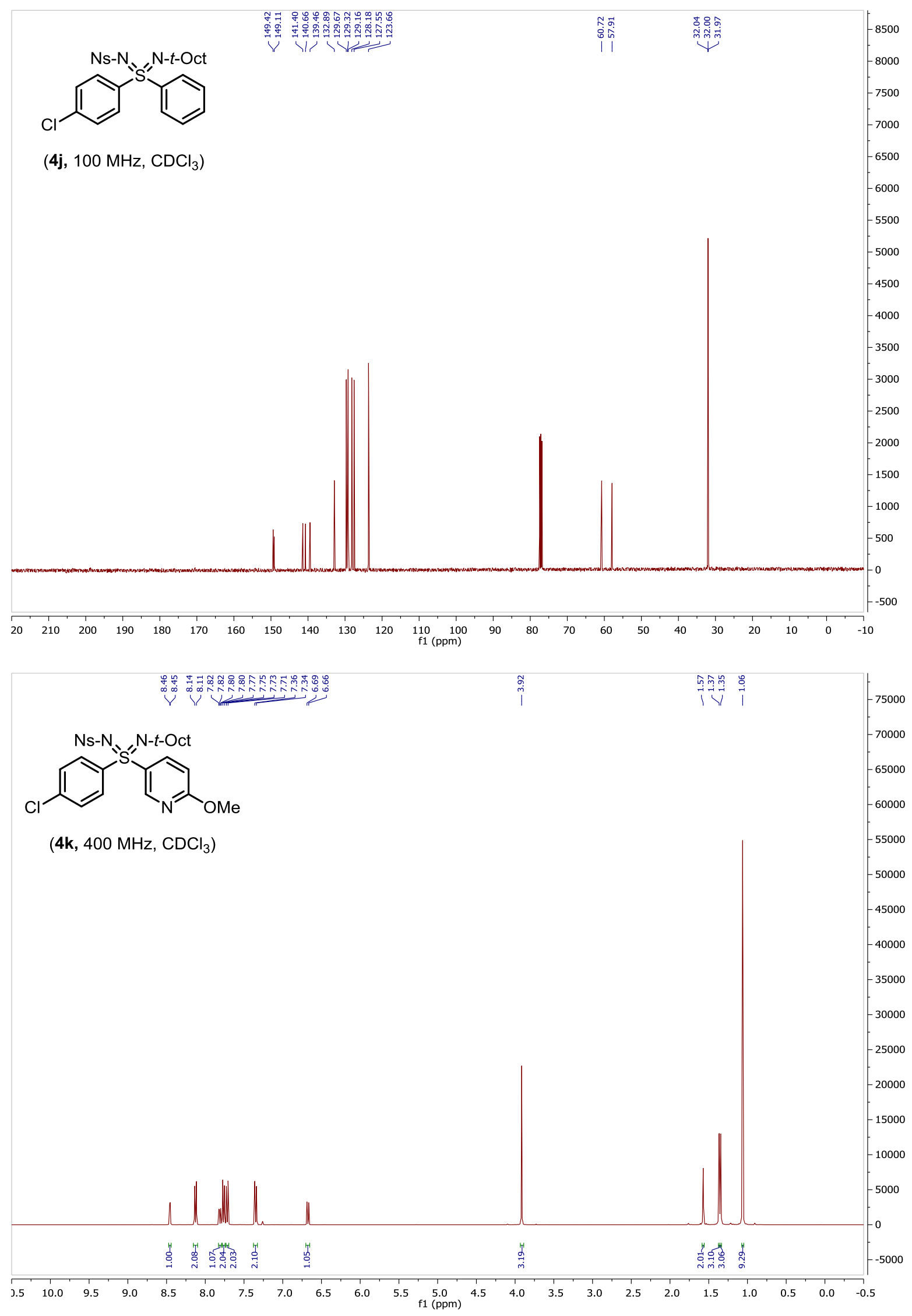



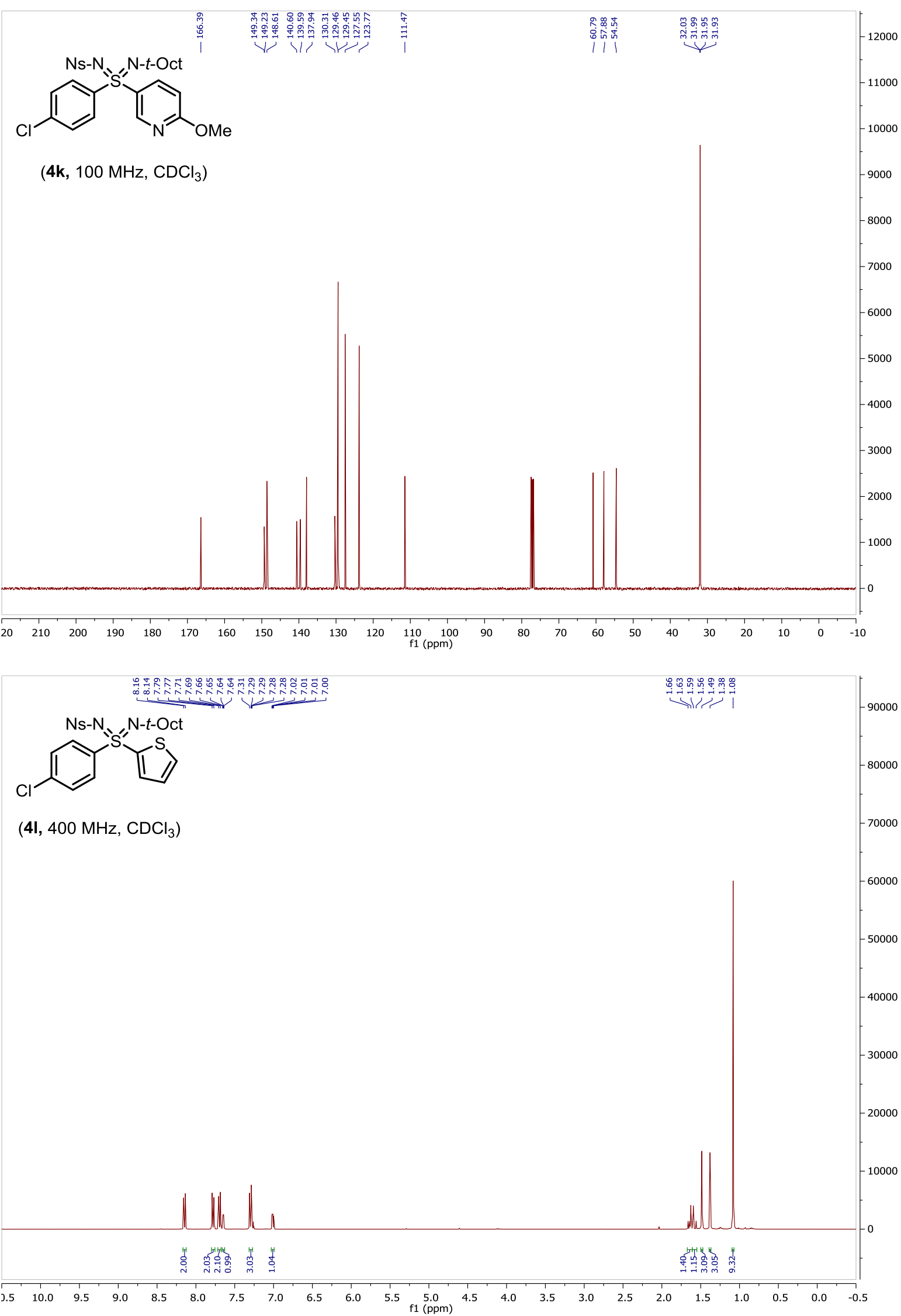

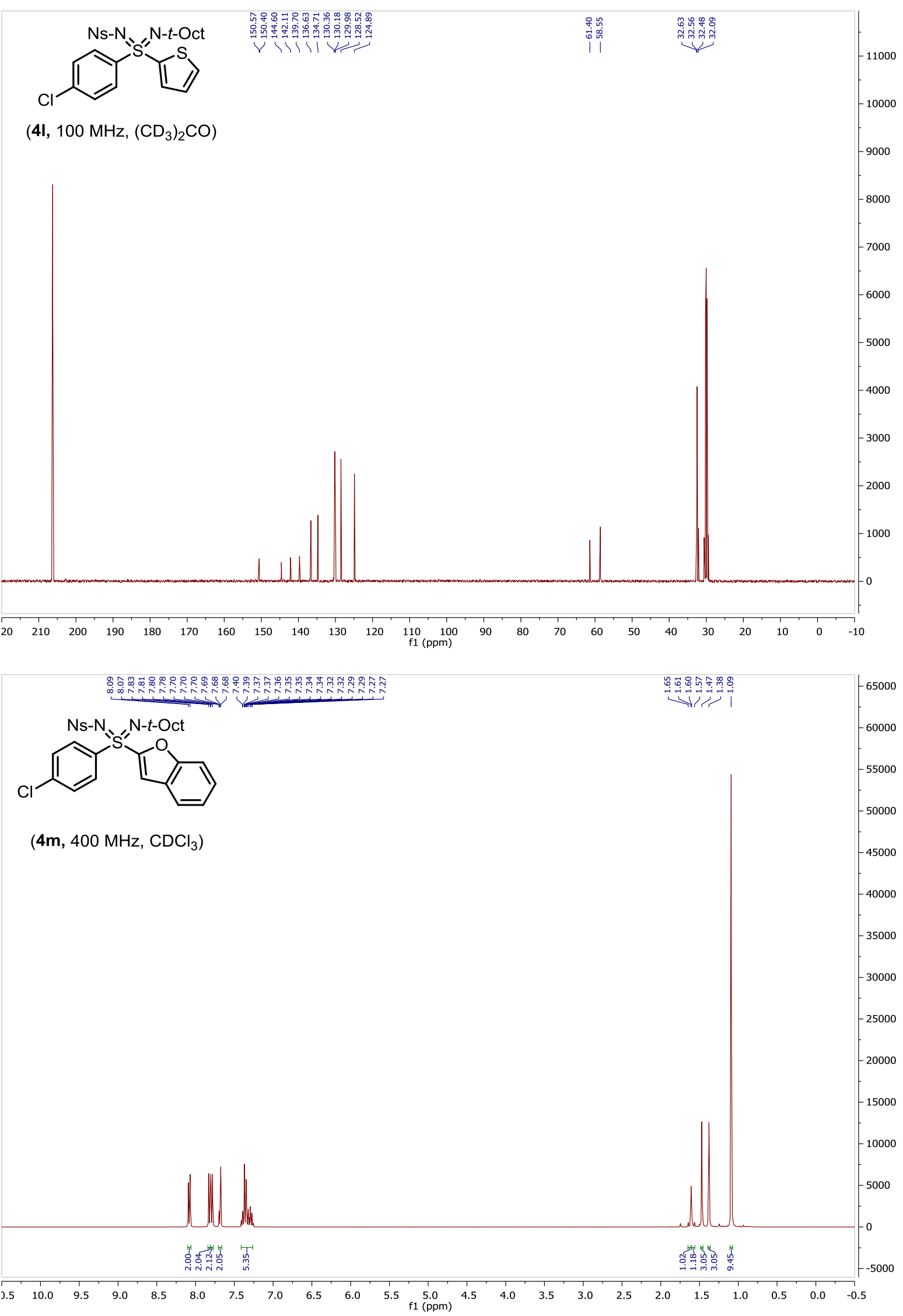

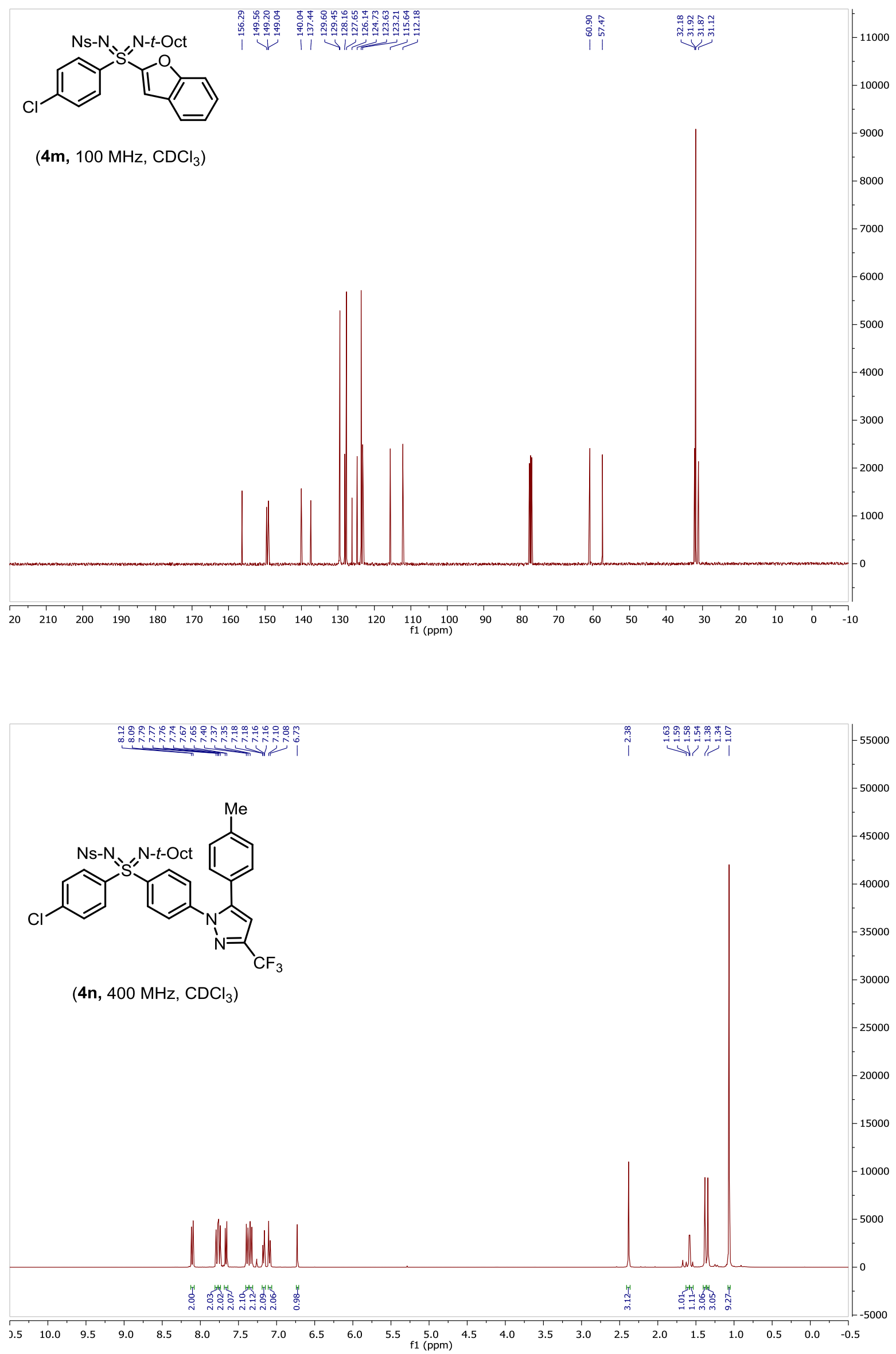

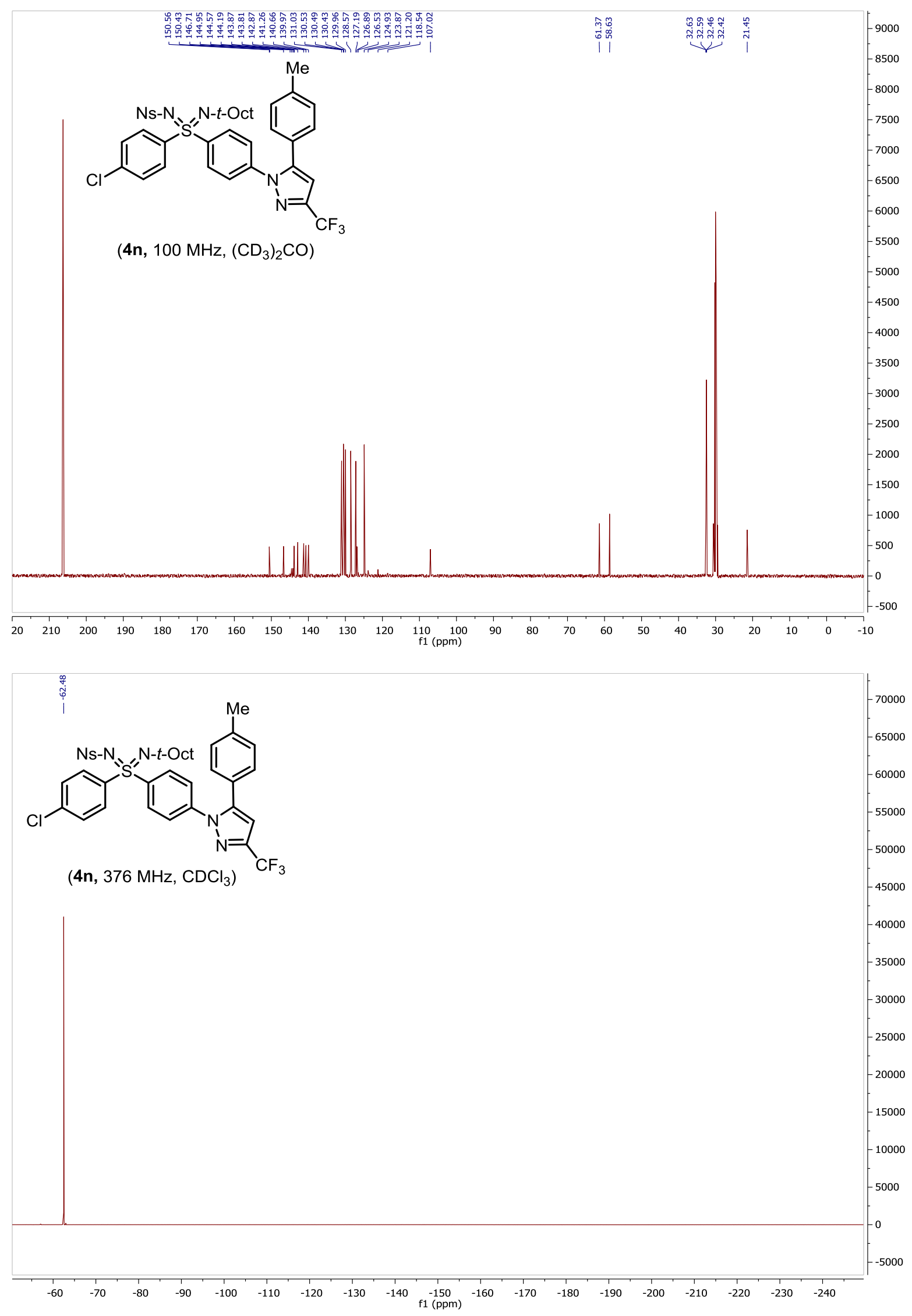


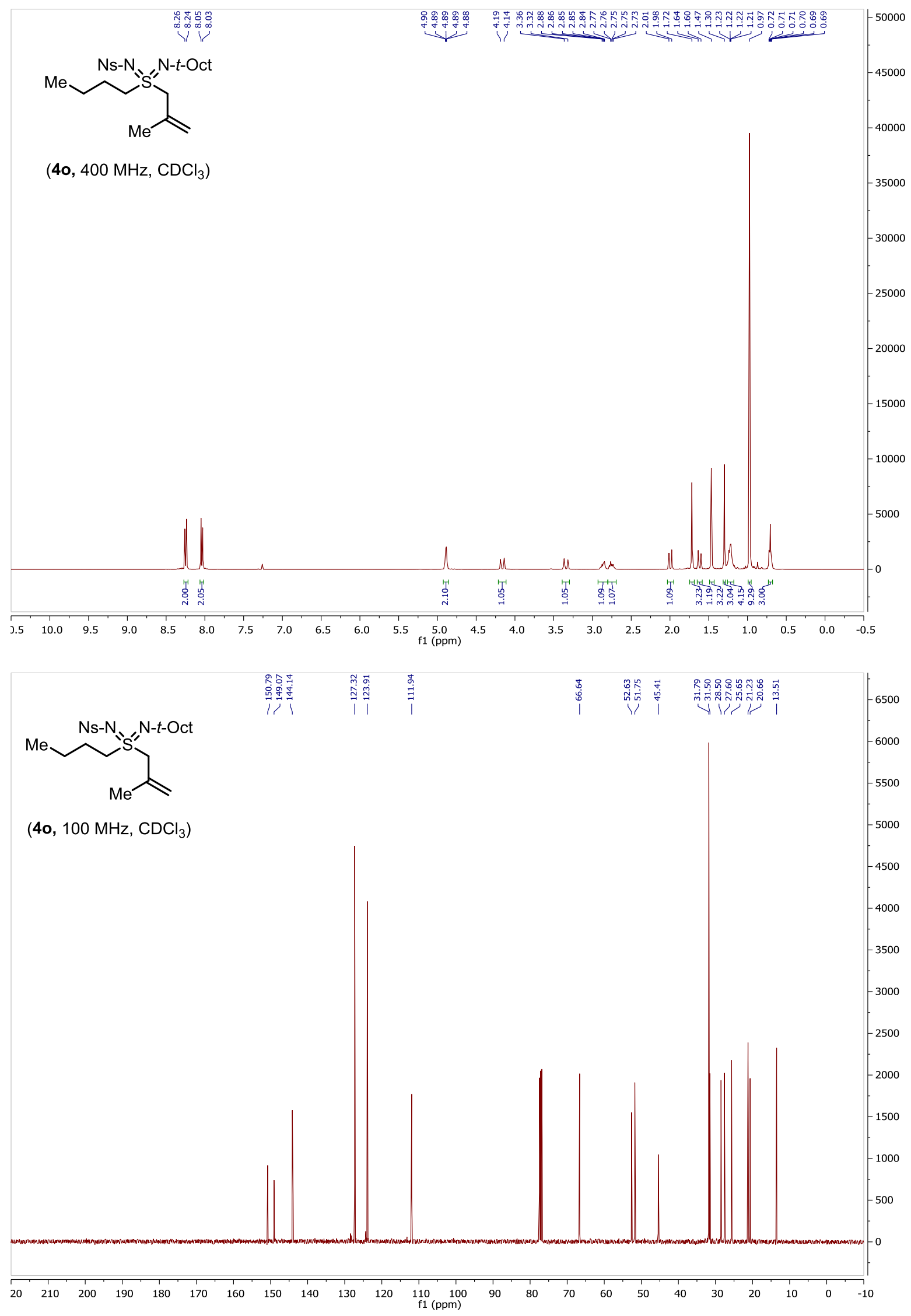



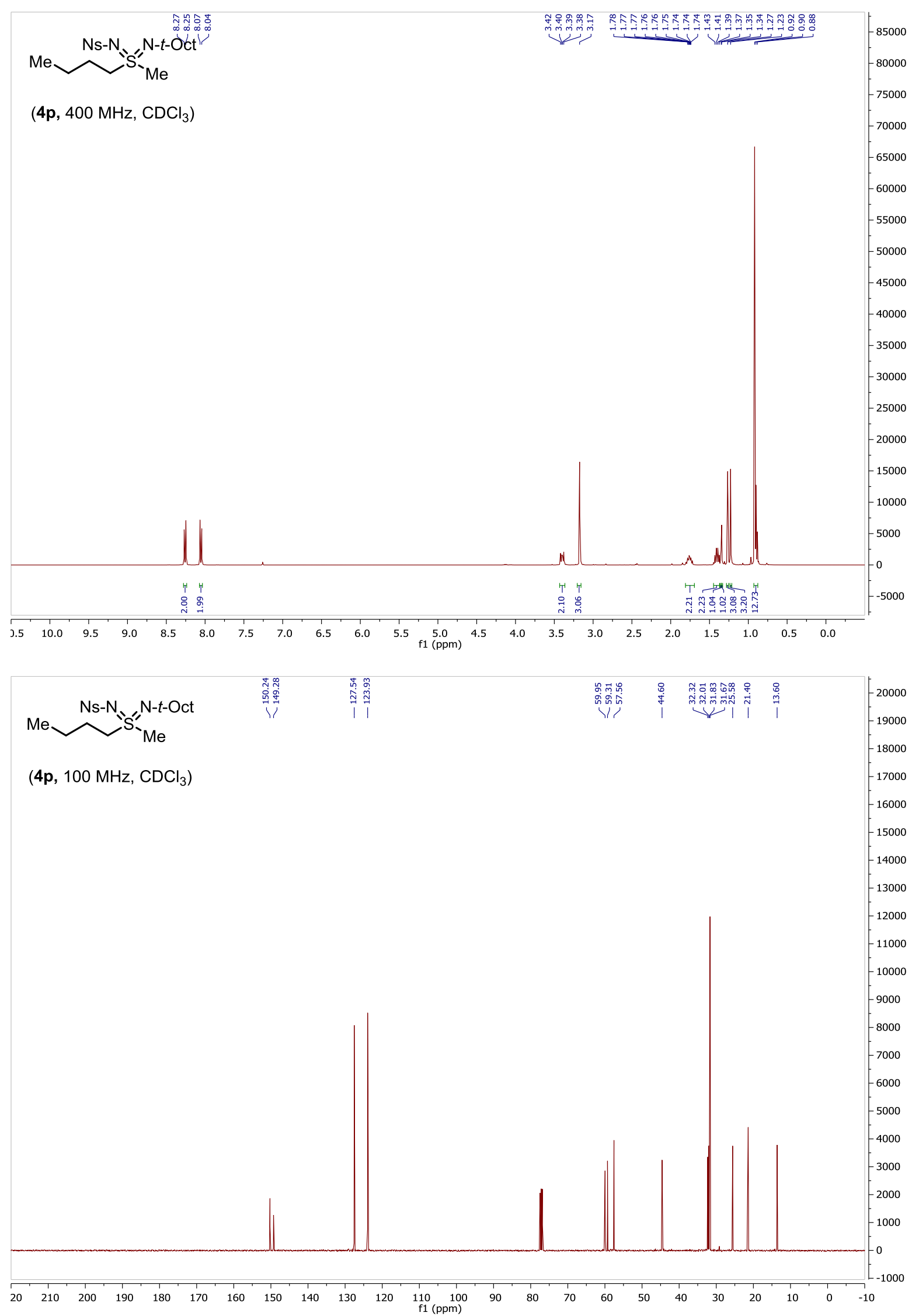


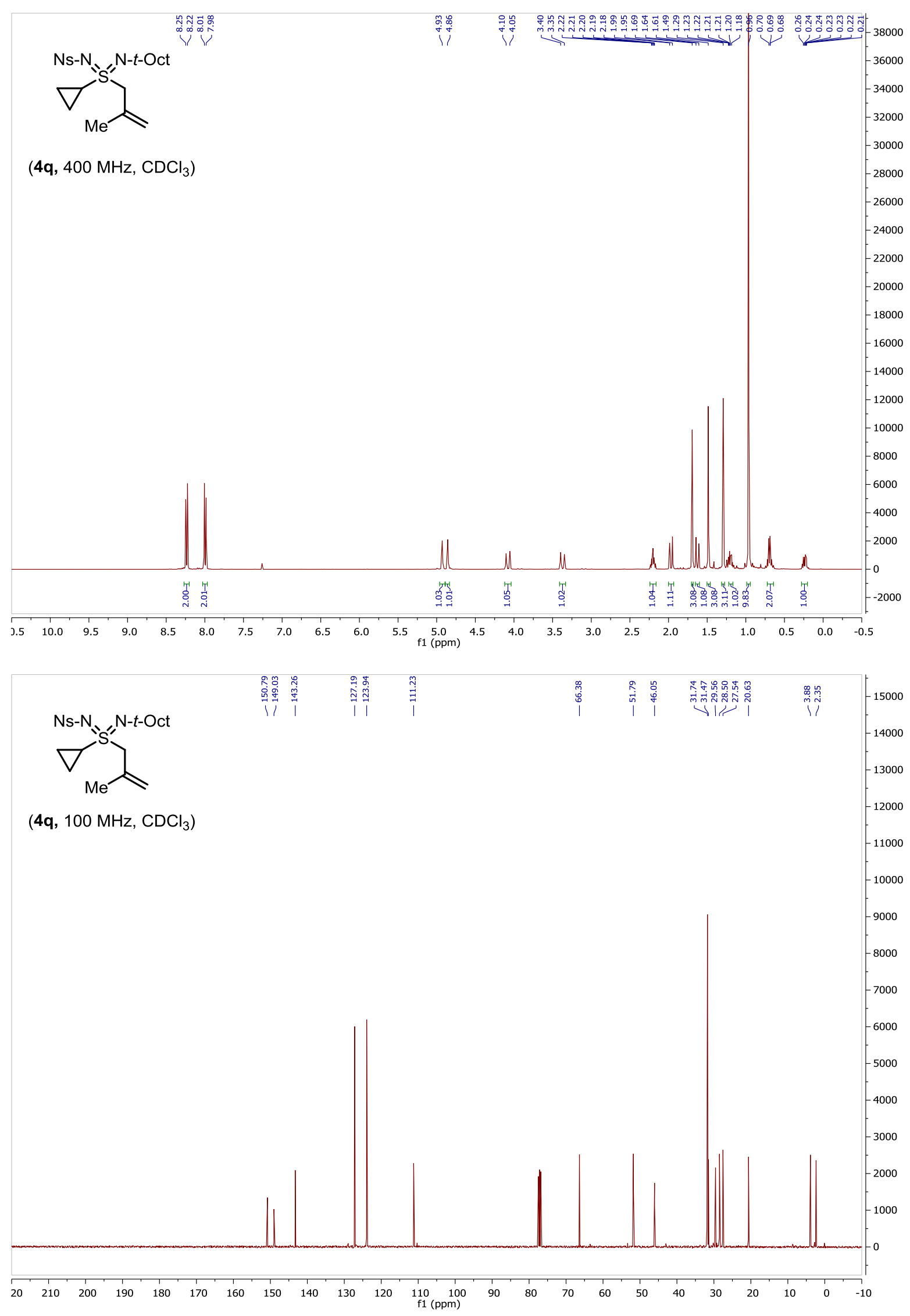




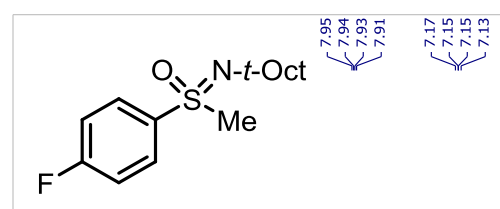

(5a, $400 \mathrm{MHz}, \mathrm{CDCl}_{3}$ )

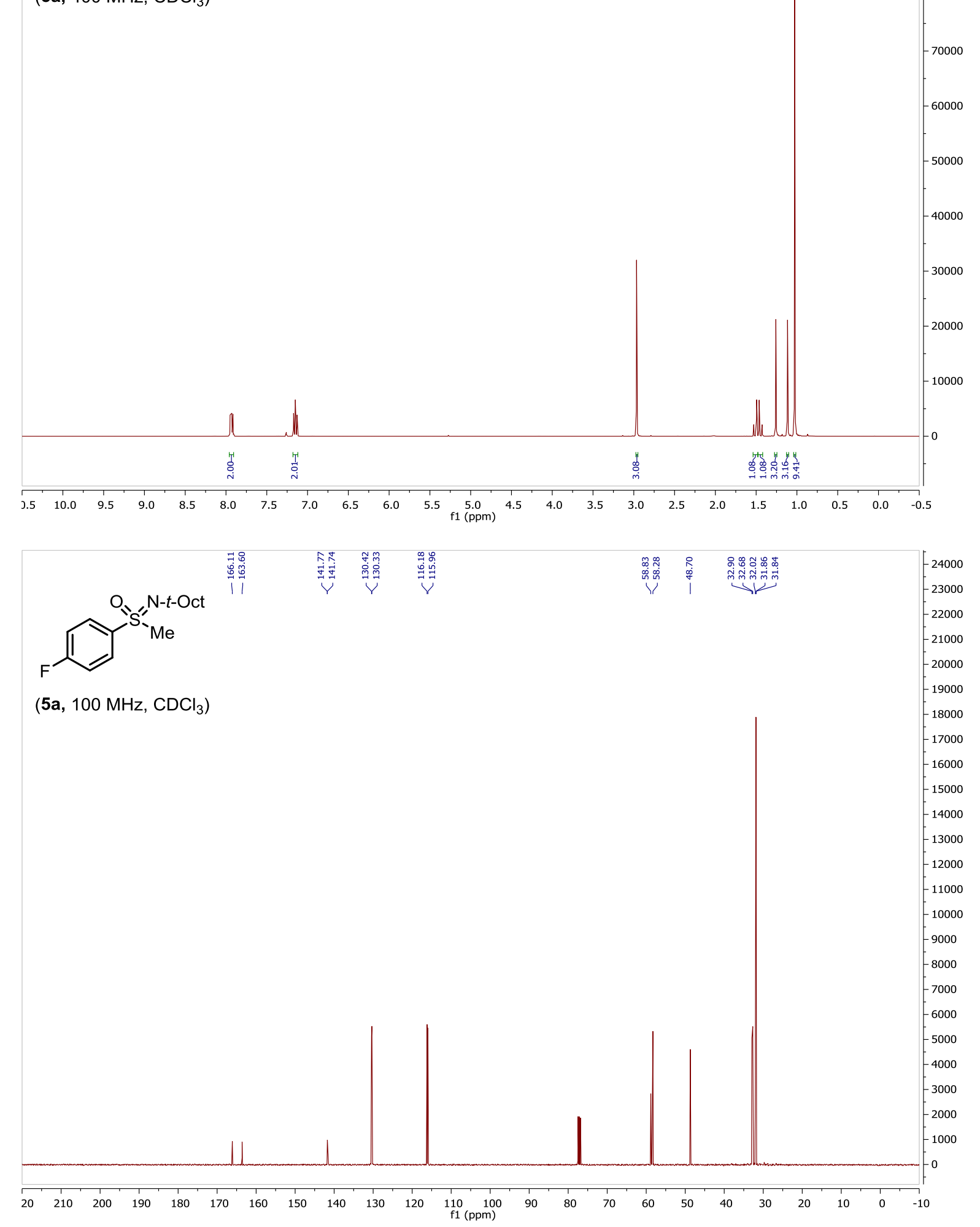



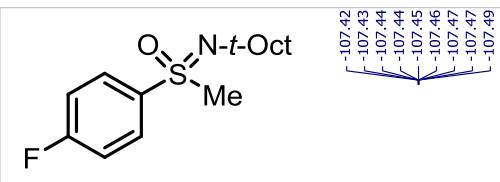

(5a, $376 \mathrm{MHz}^{\mathrm{CDCl}_{3}}$ )

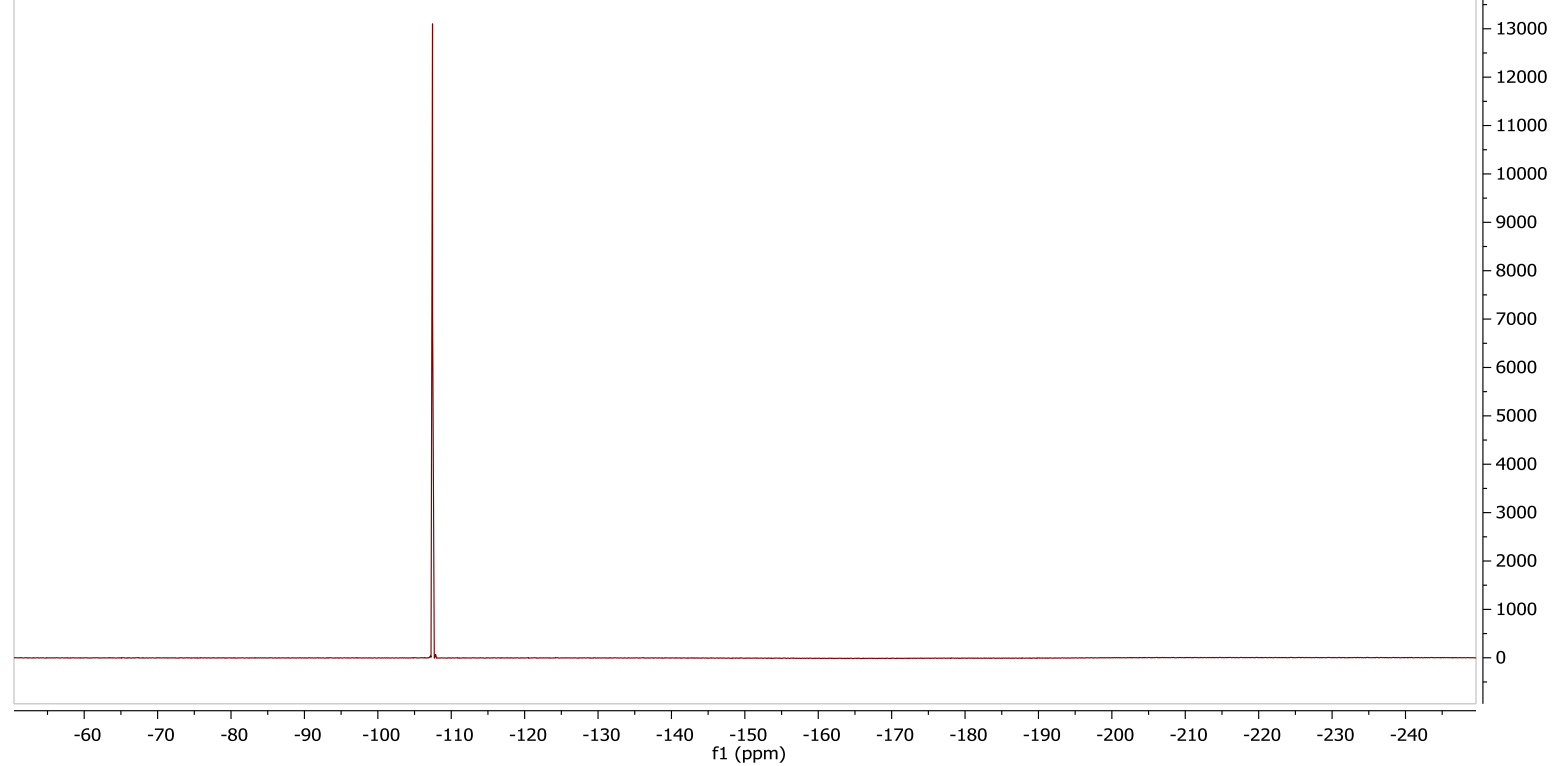

1000

10000

000

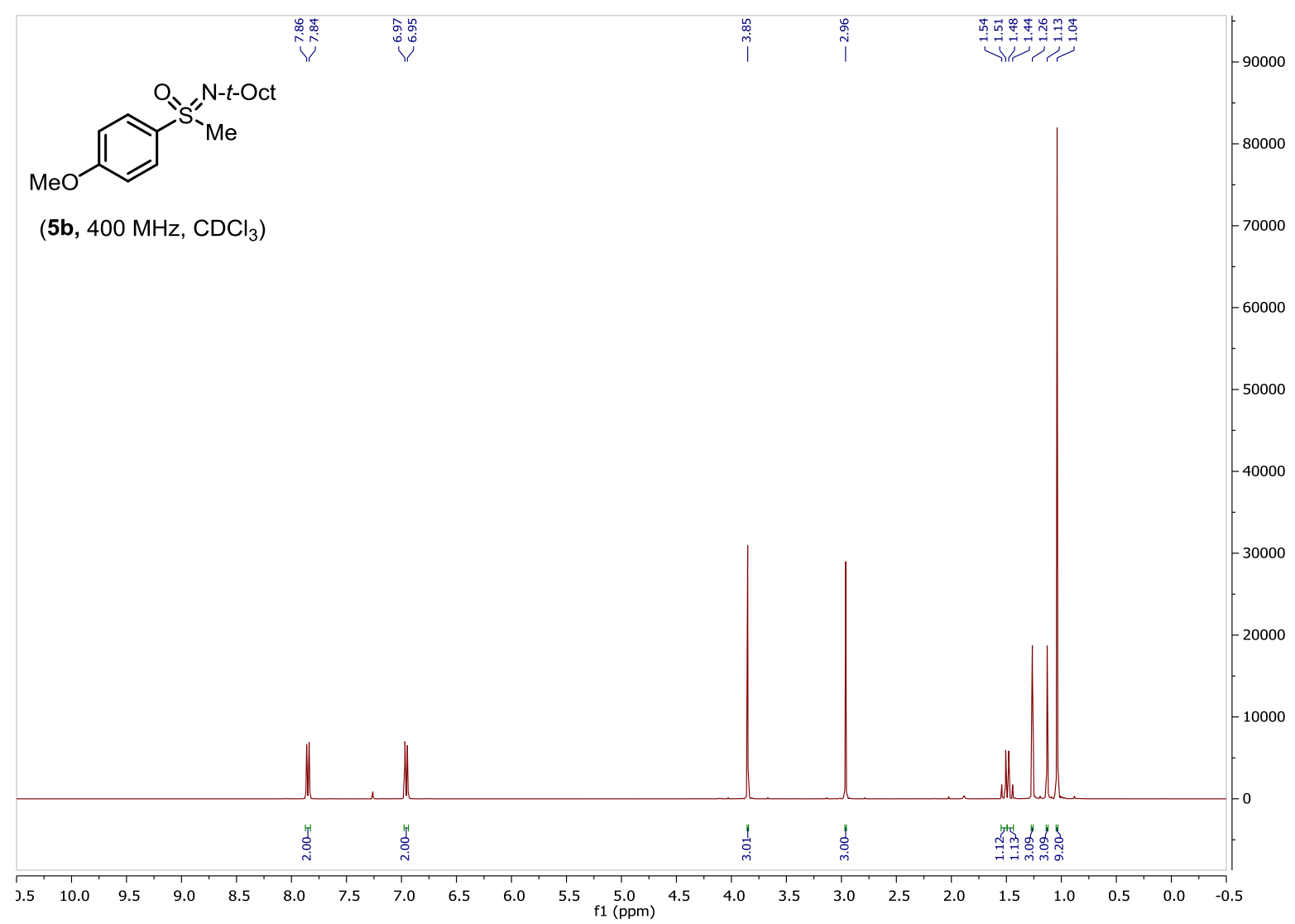



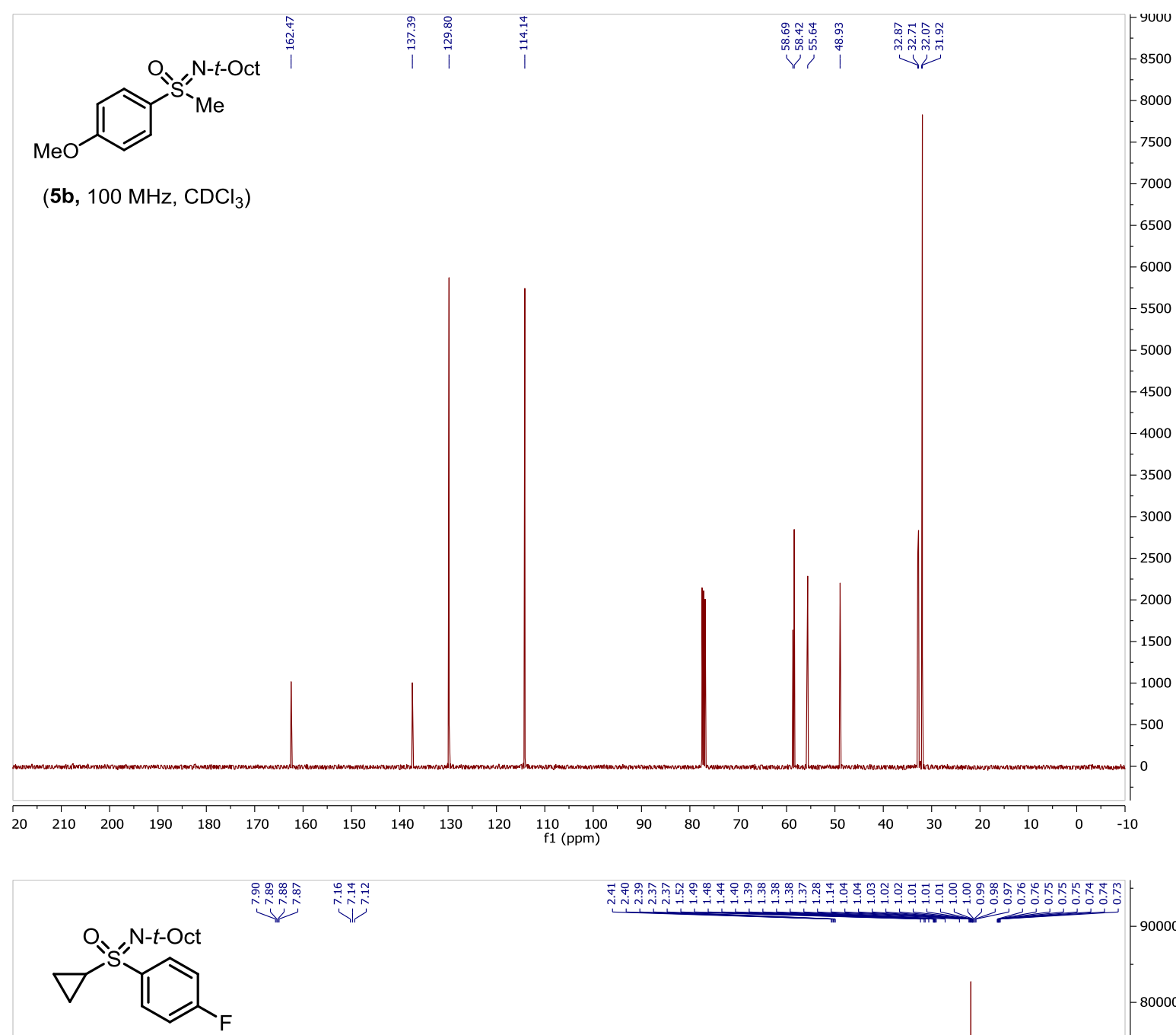

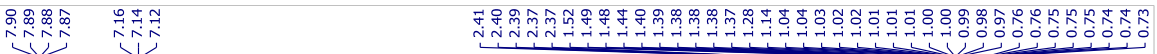

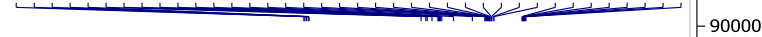

(5c, $400 \mathrm{MHz}, \mathrm{CDCl}_{3}$ )

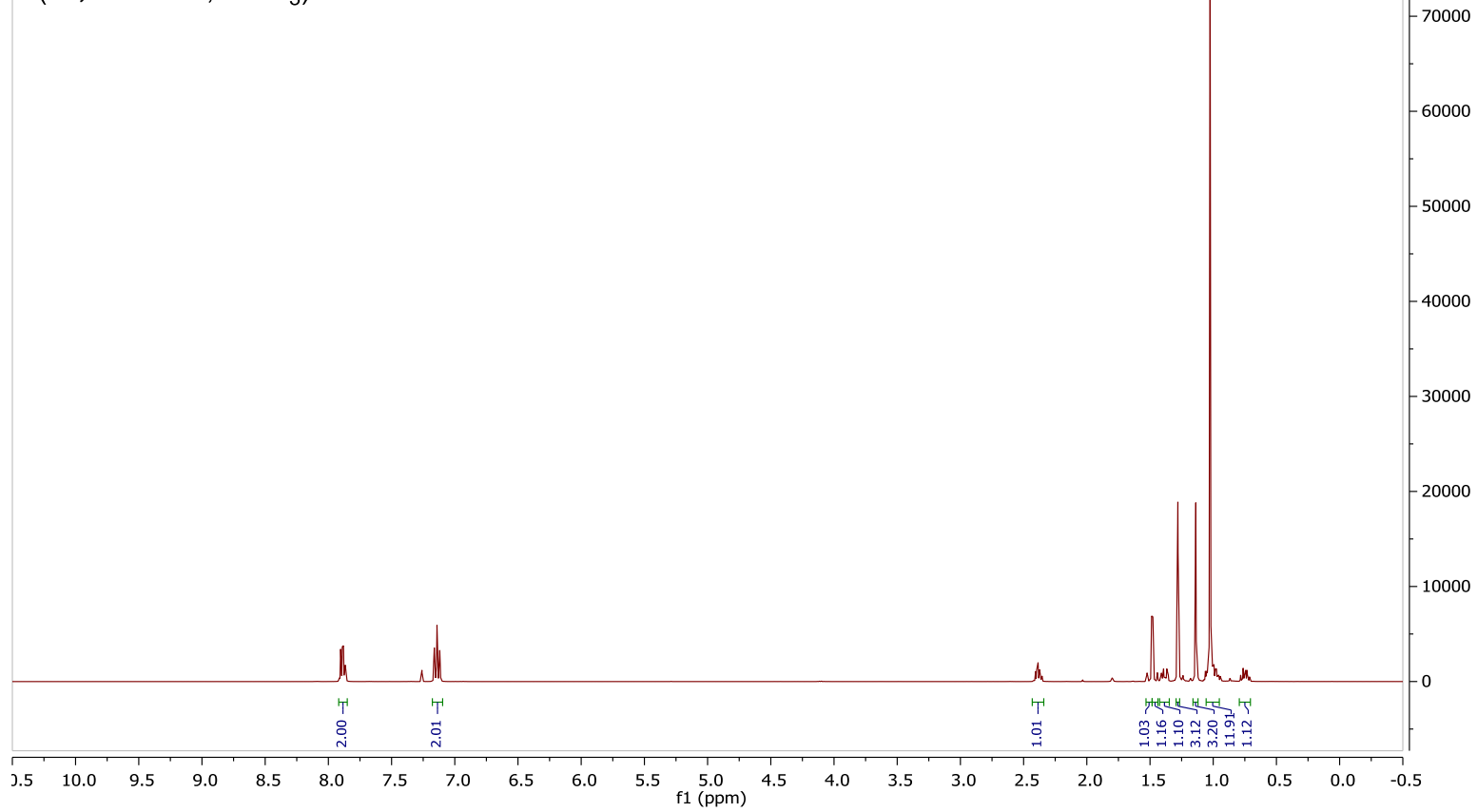



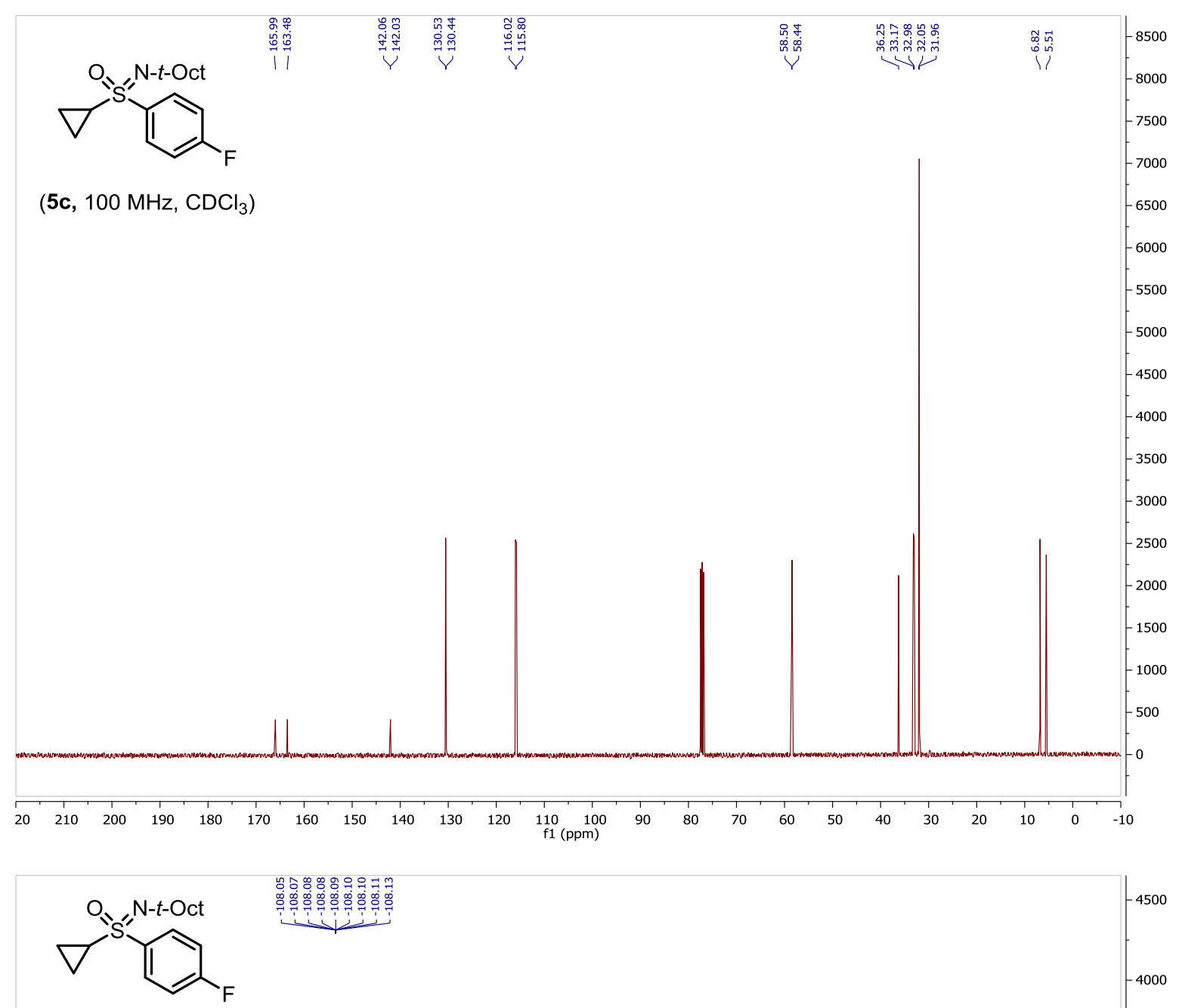

(5c, $376 \mathrm{MHz}, \mathrm{CDCl}_{3}$ )

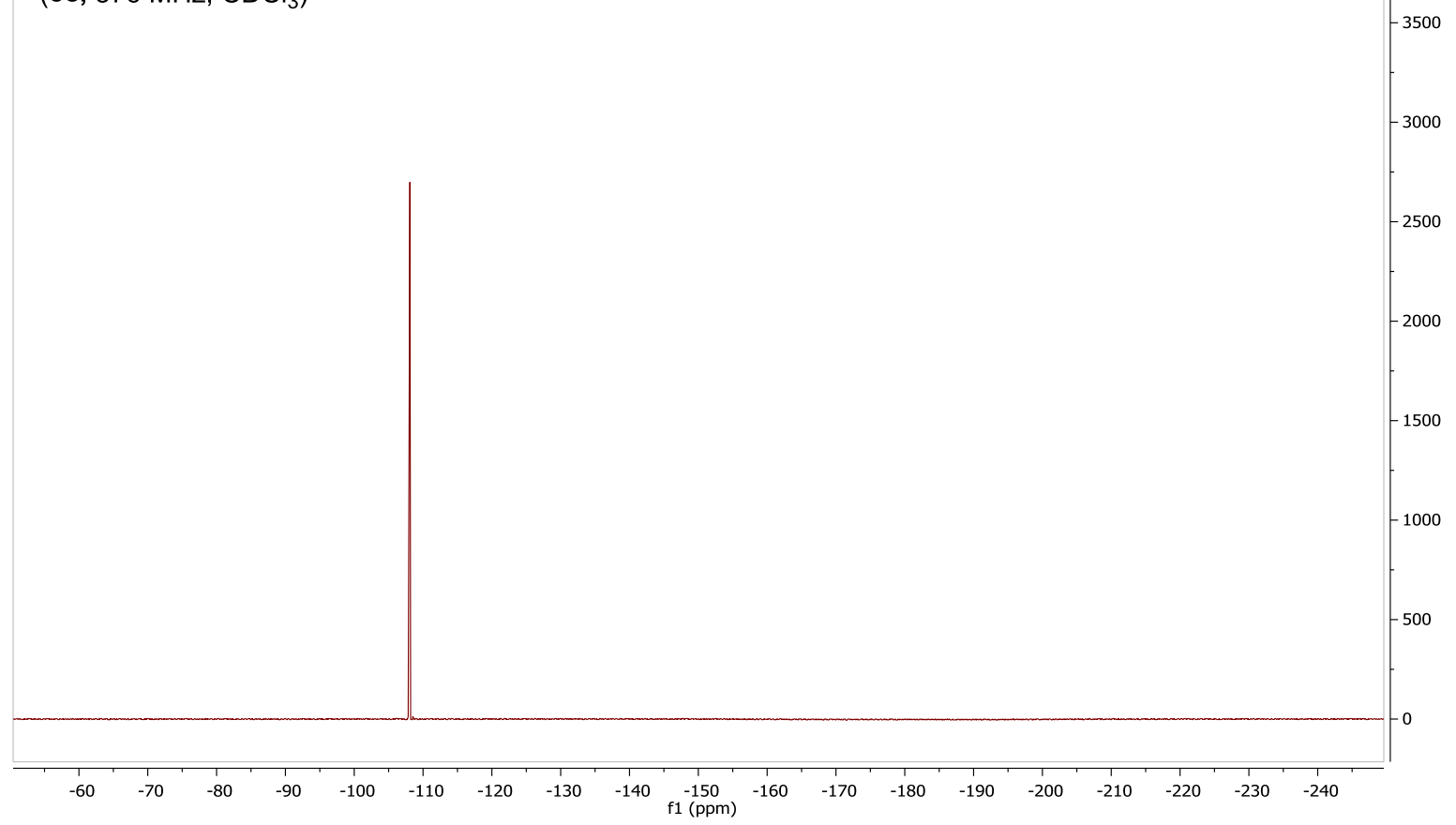




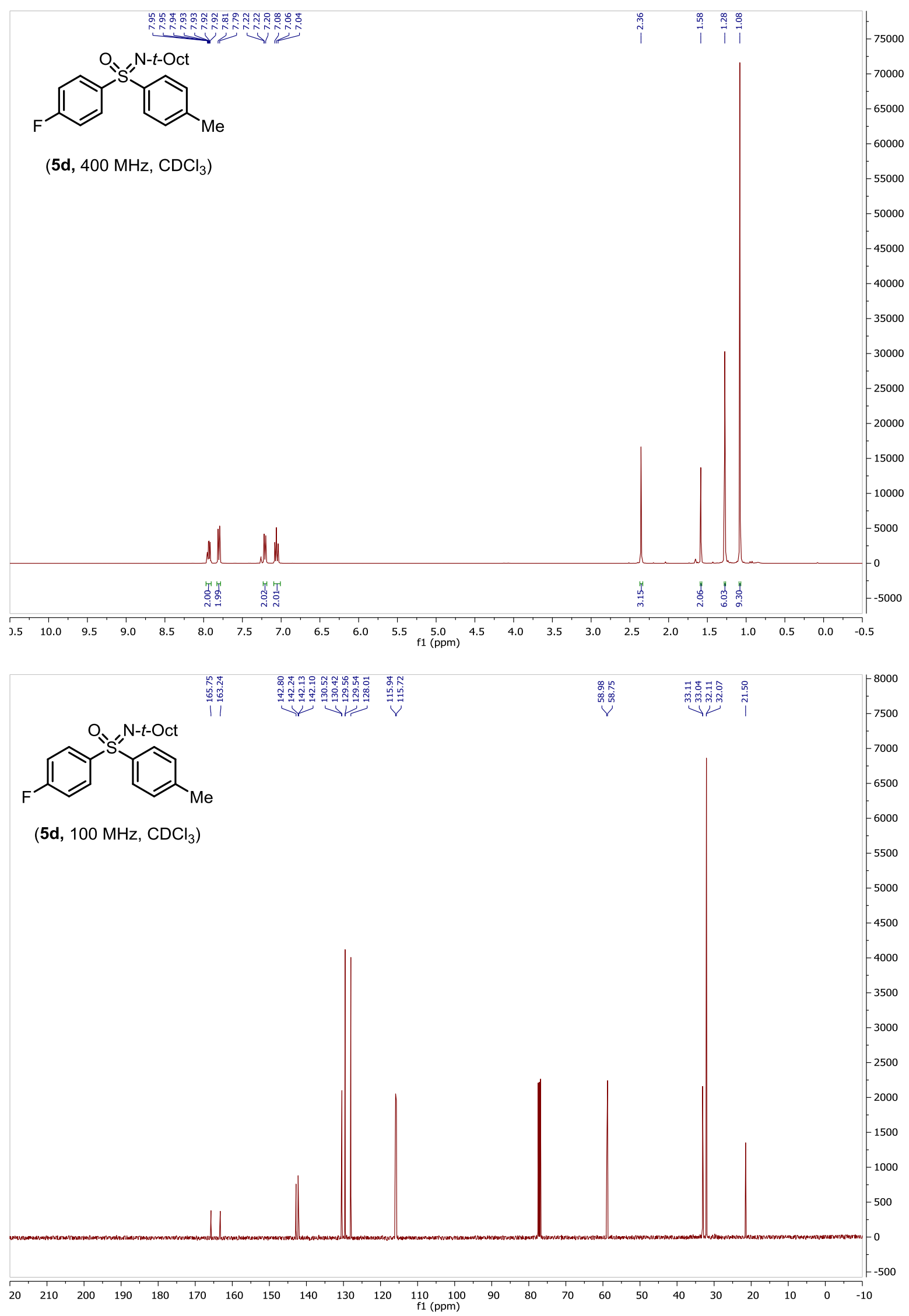



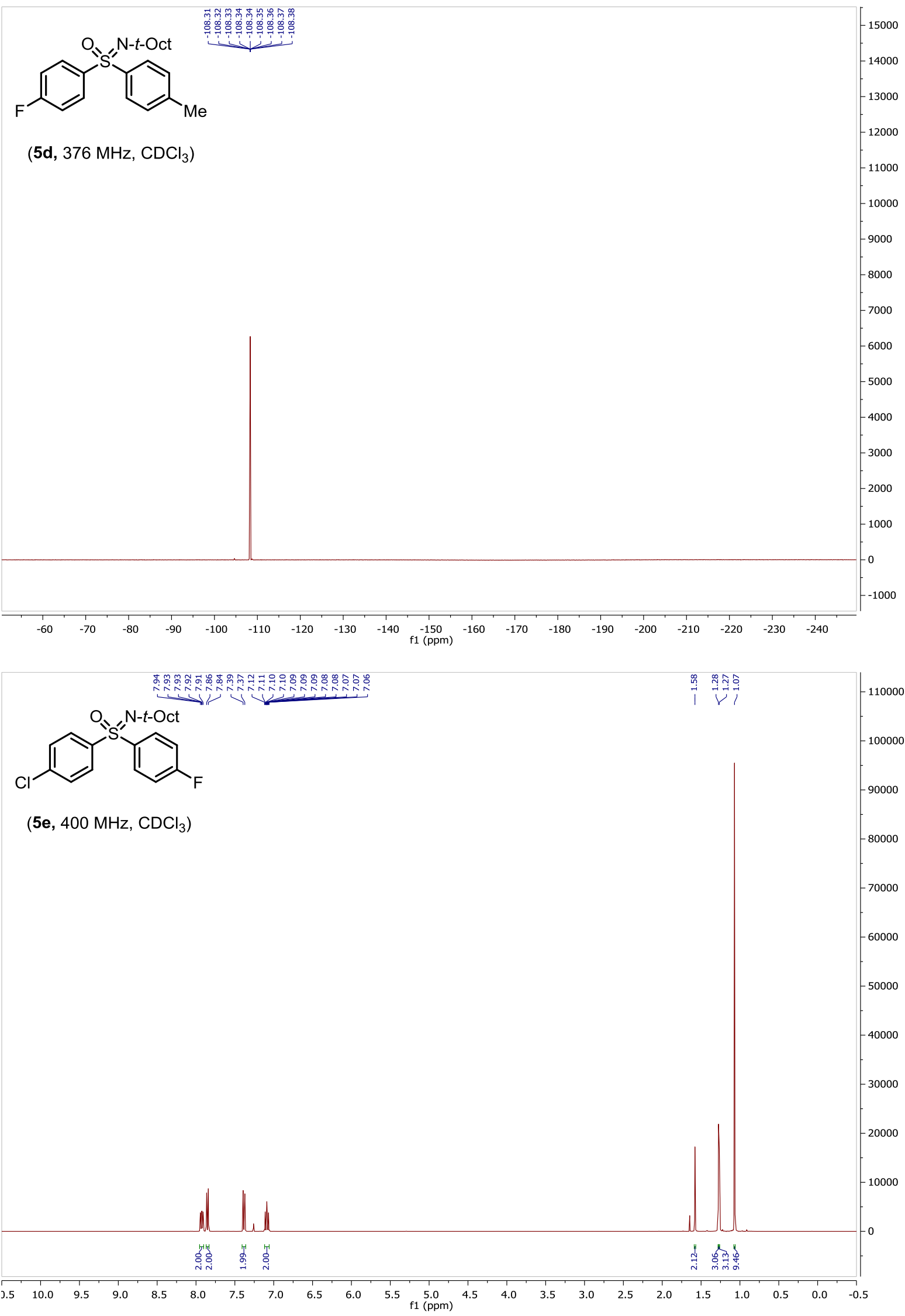

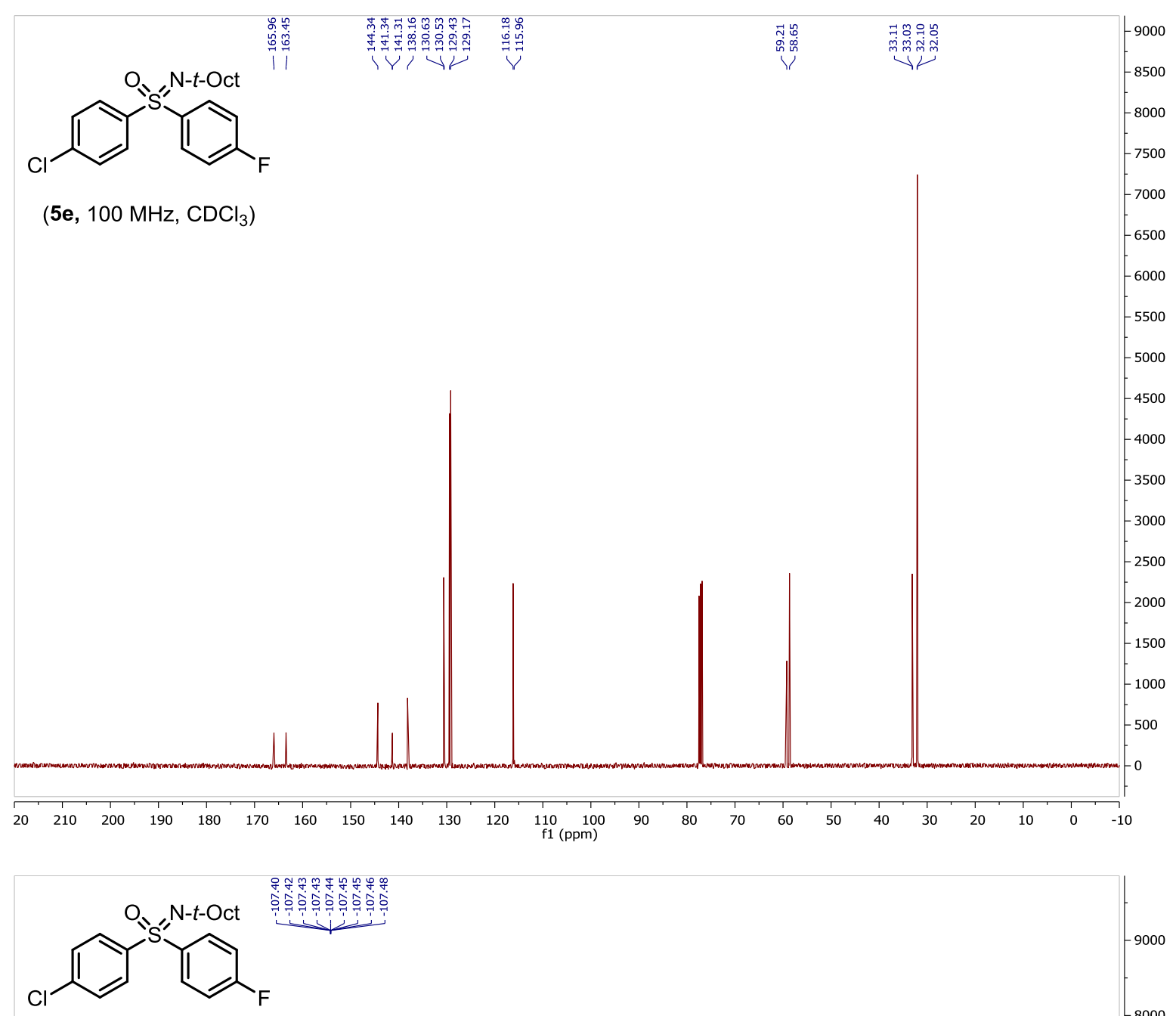

(5e, $376 \mathrm{MHz} \mathrm{CDCl}_{3}$ )

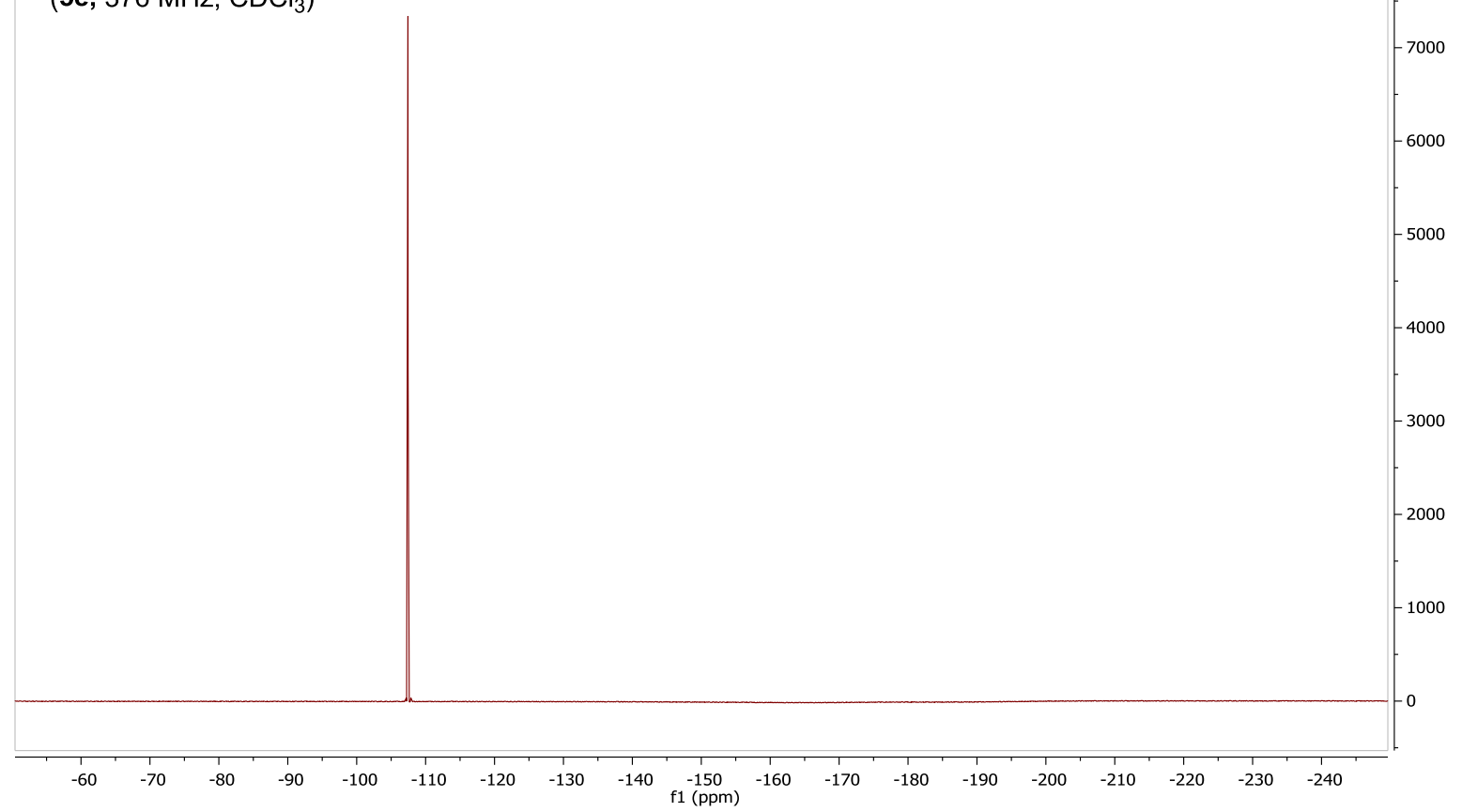




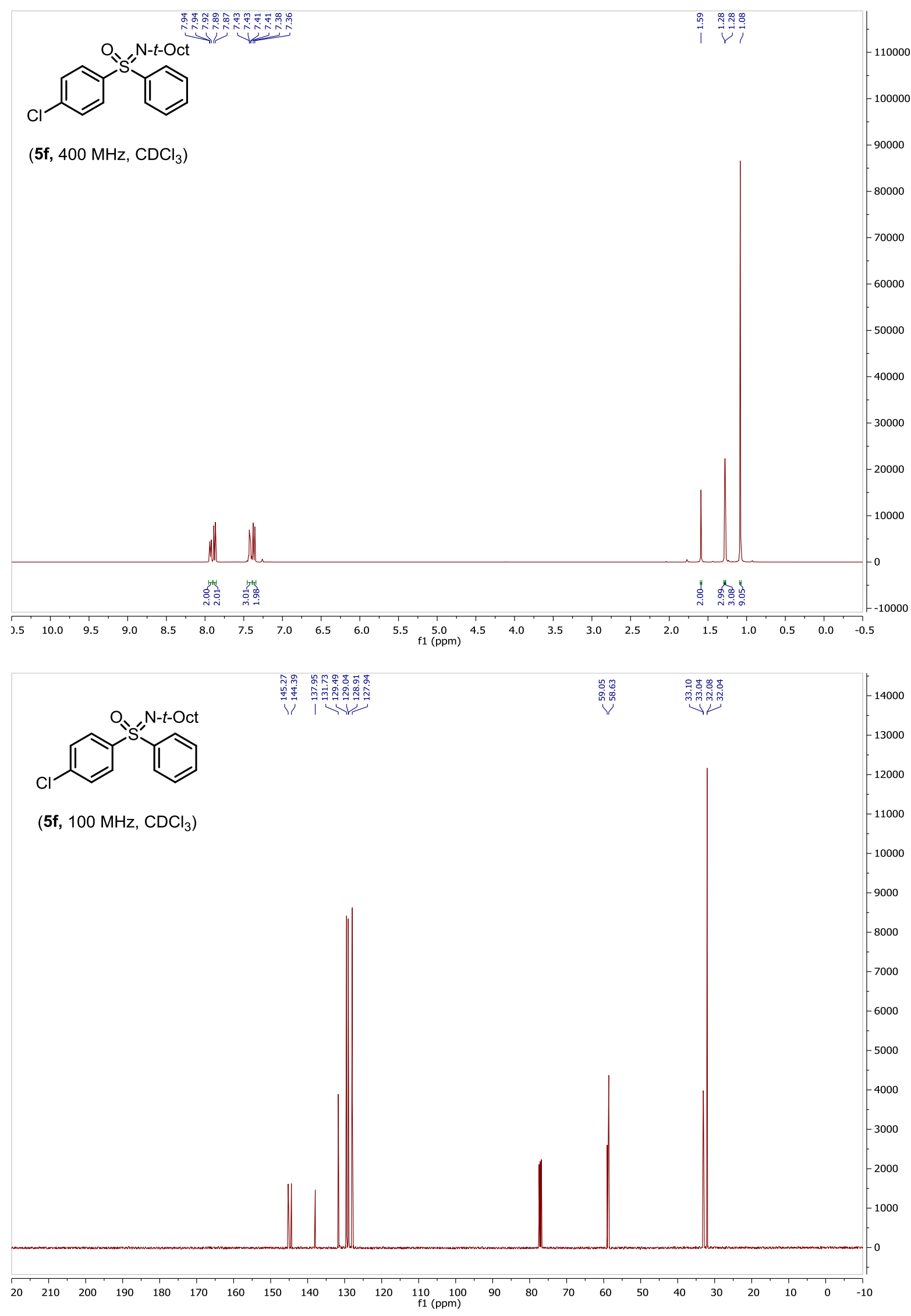




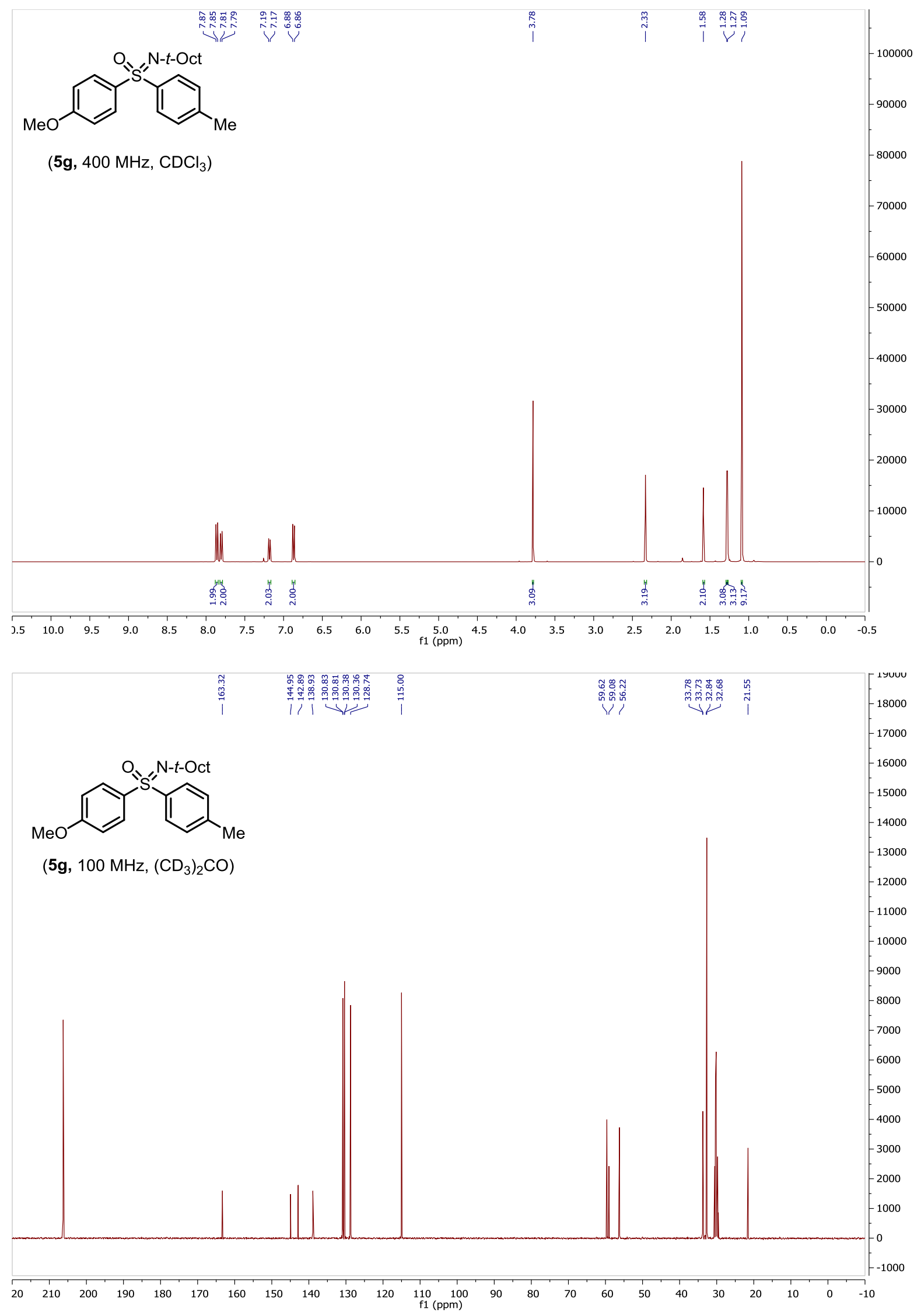



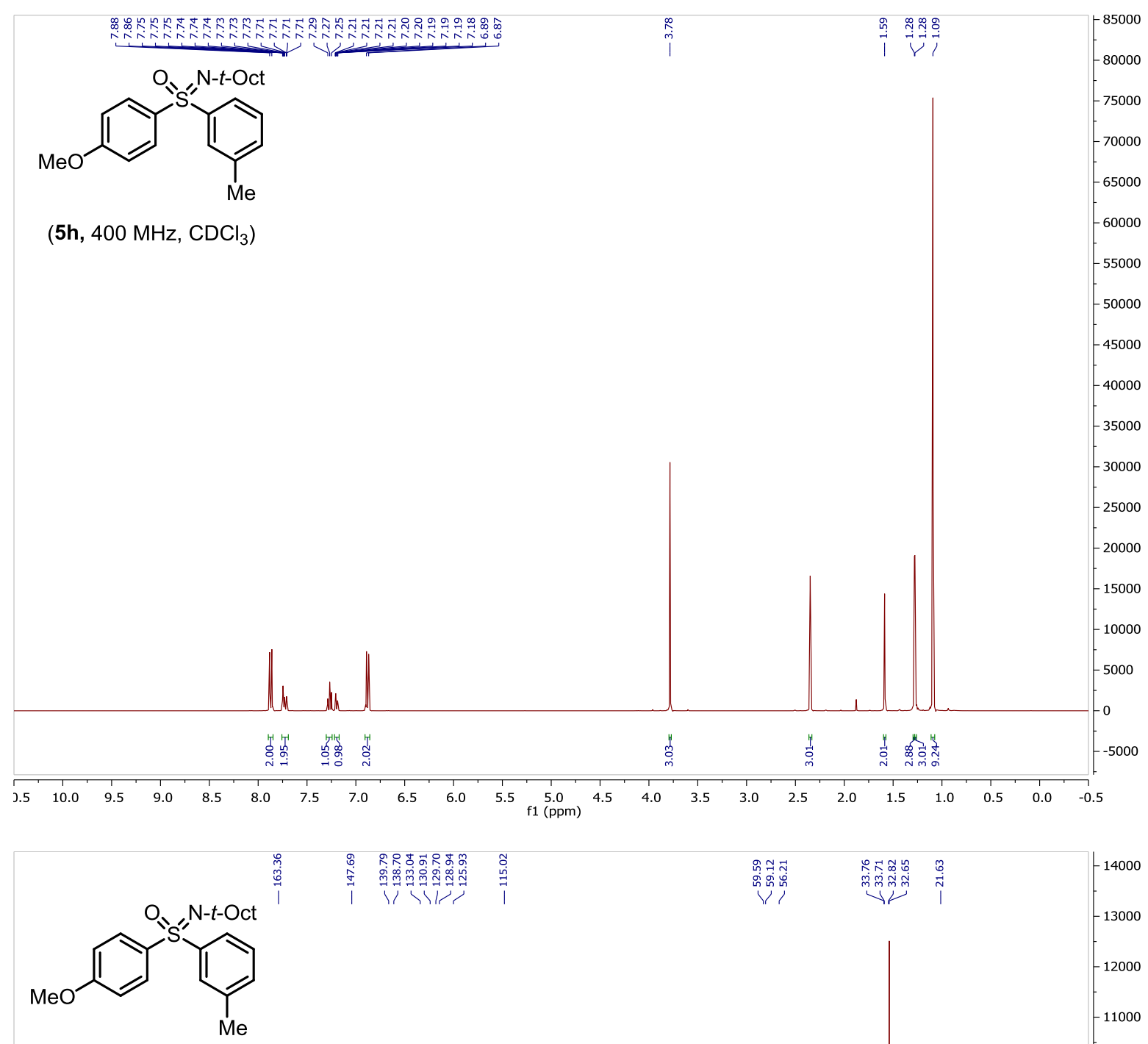

(5h, $\left.100 \mathrm{MHz},\left(\mathrm{CD}_{3}\right)_{2} \mathrm{CO}\right)$

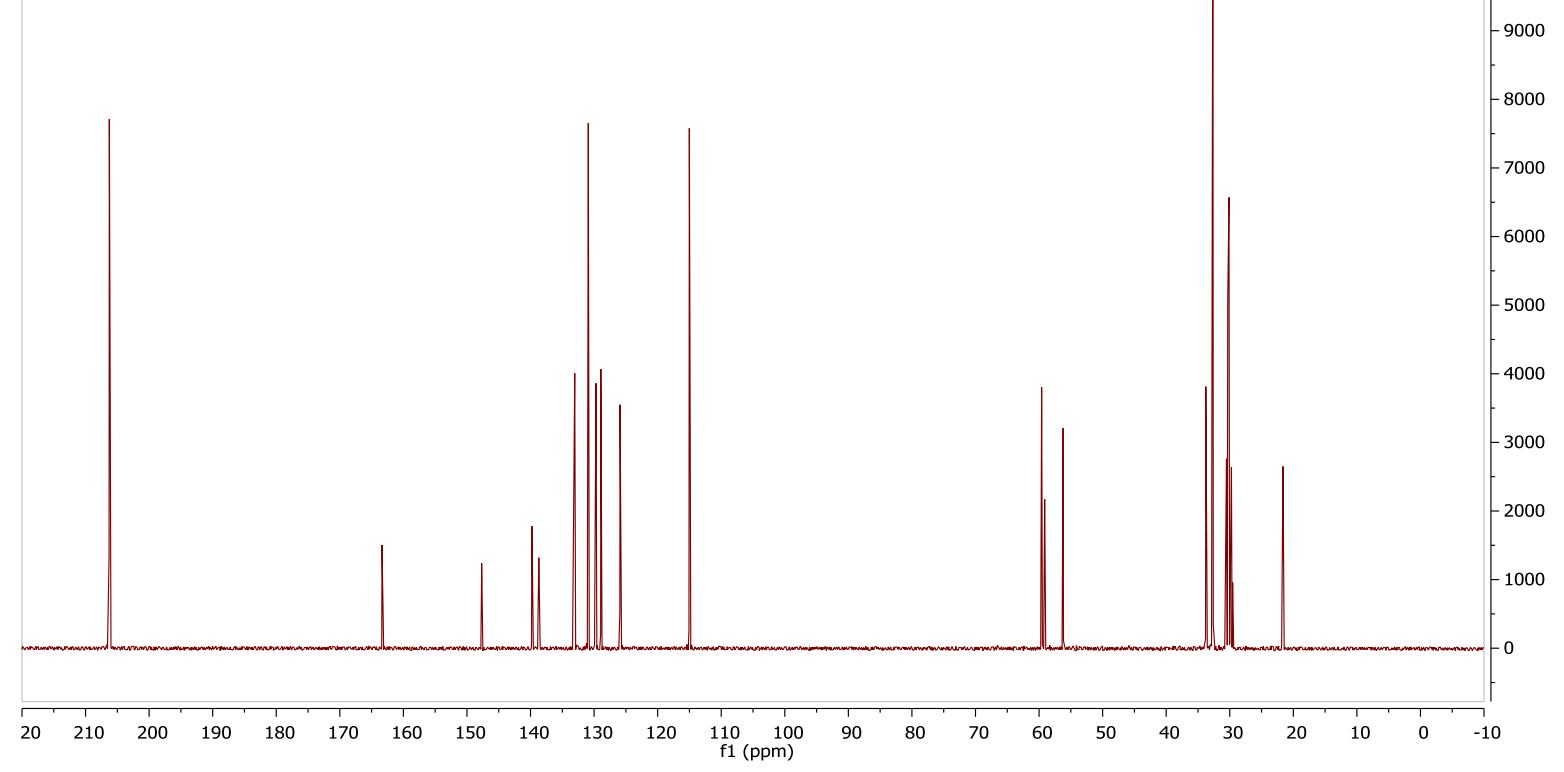



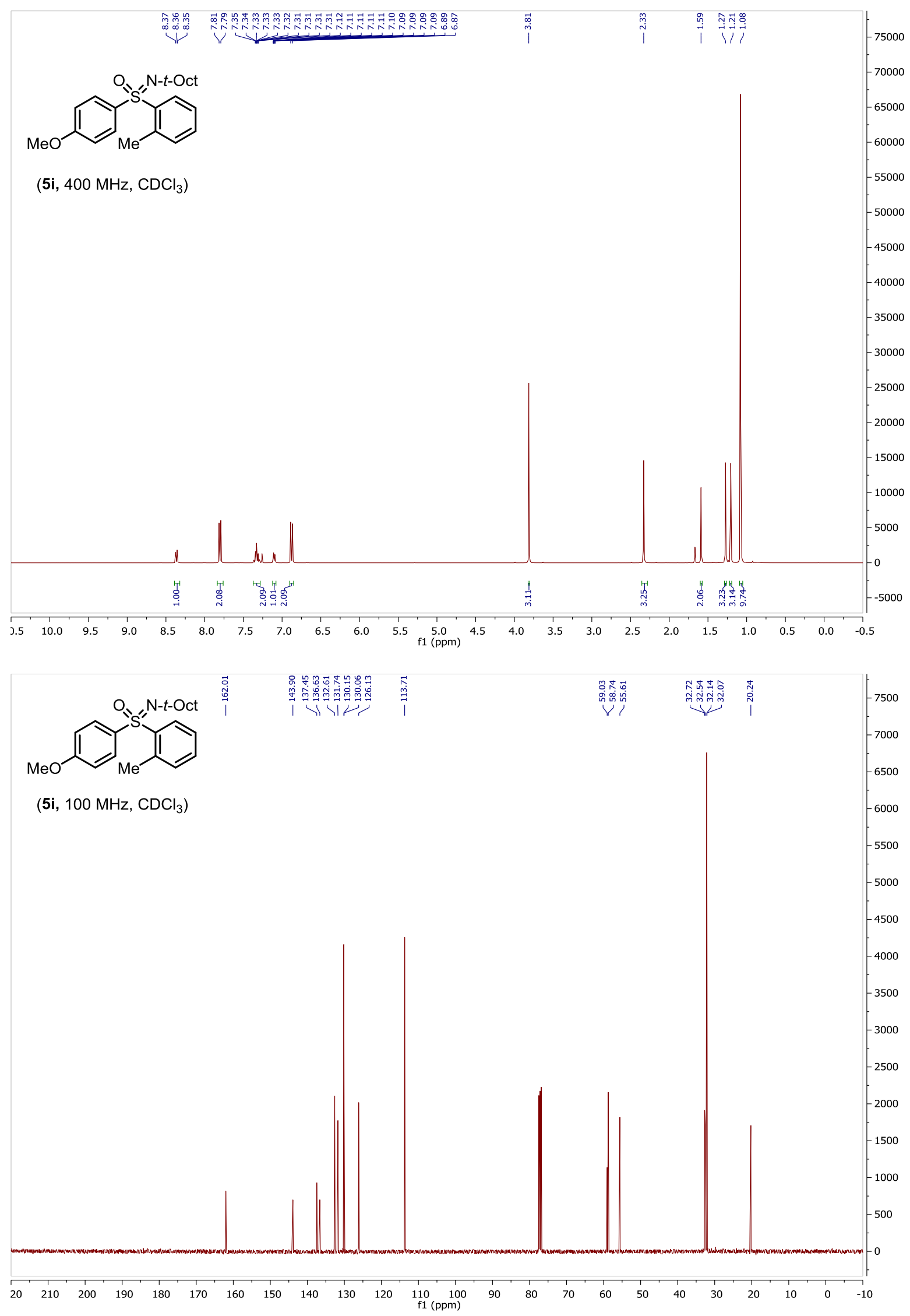

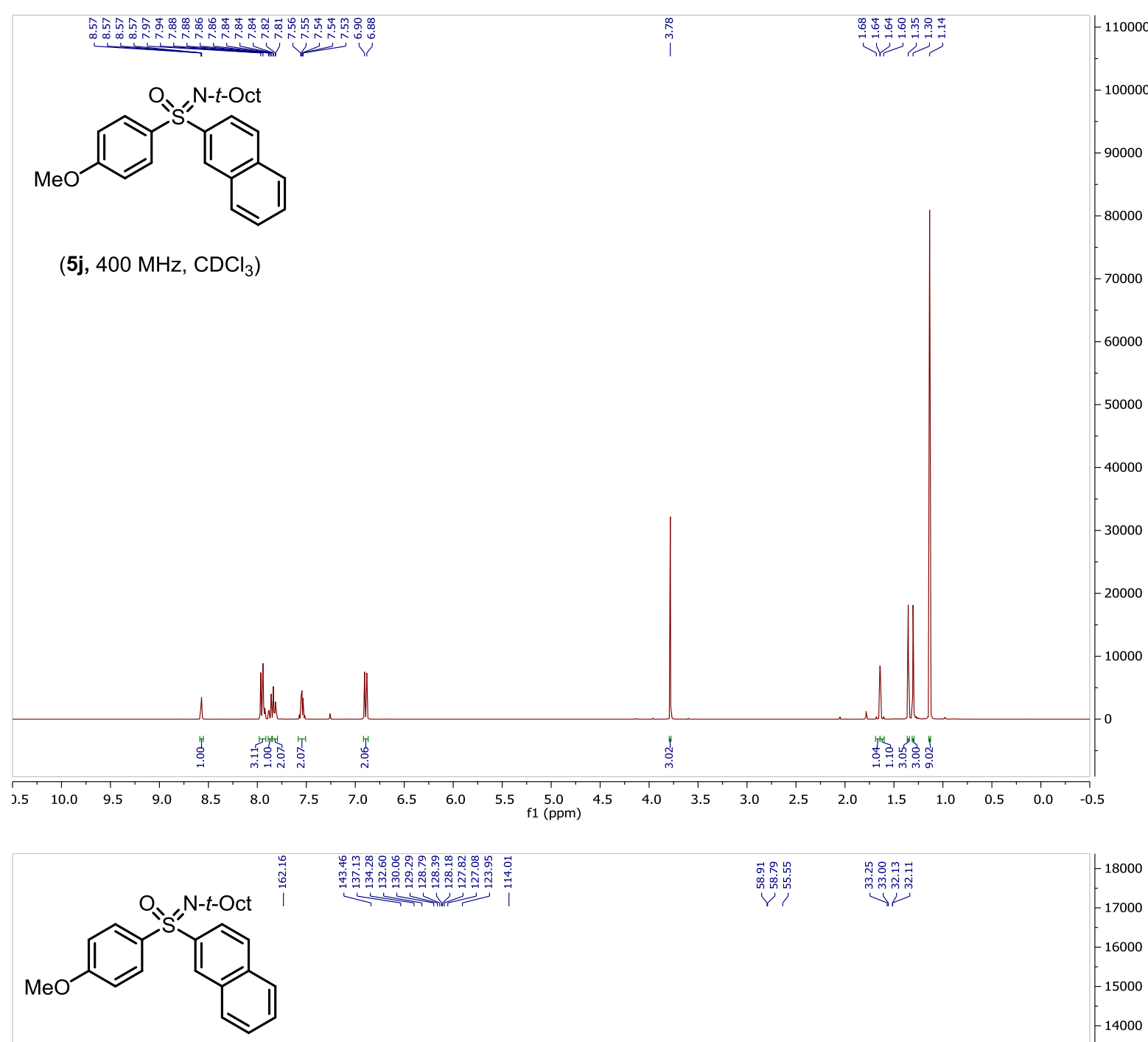

(5j, $100 \mathrm{MHz}, \mathrm{CDCl}_{3}$ )

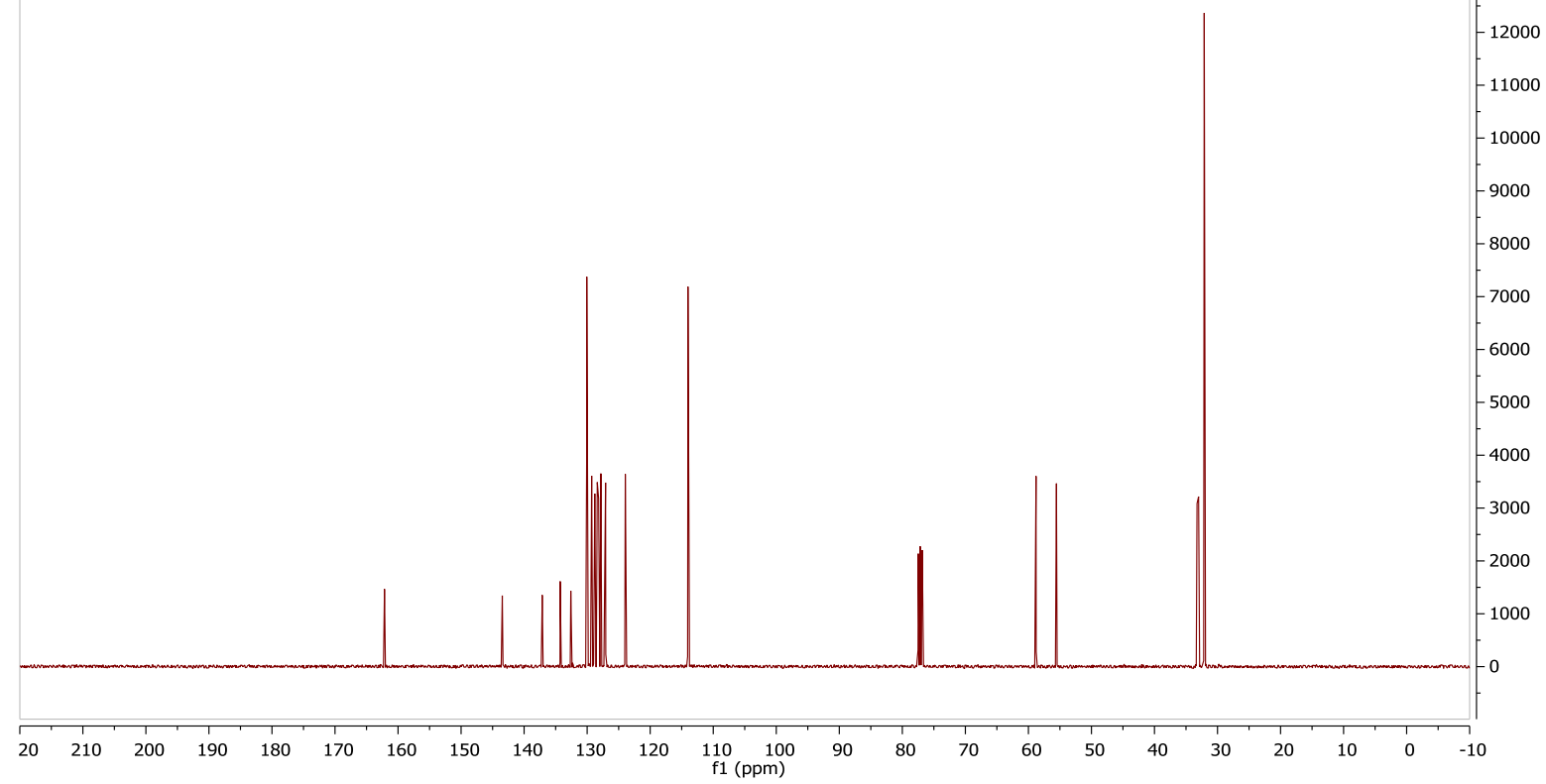



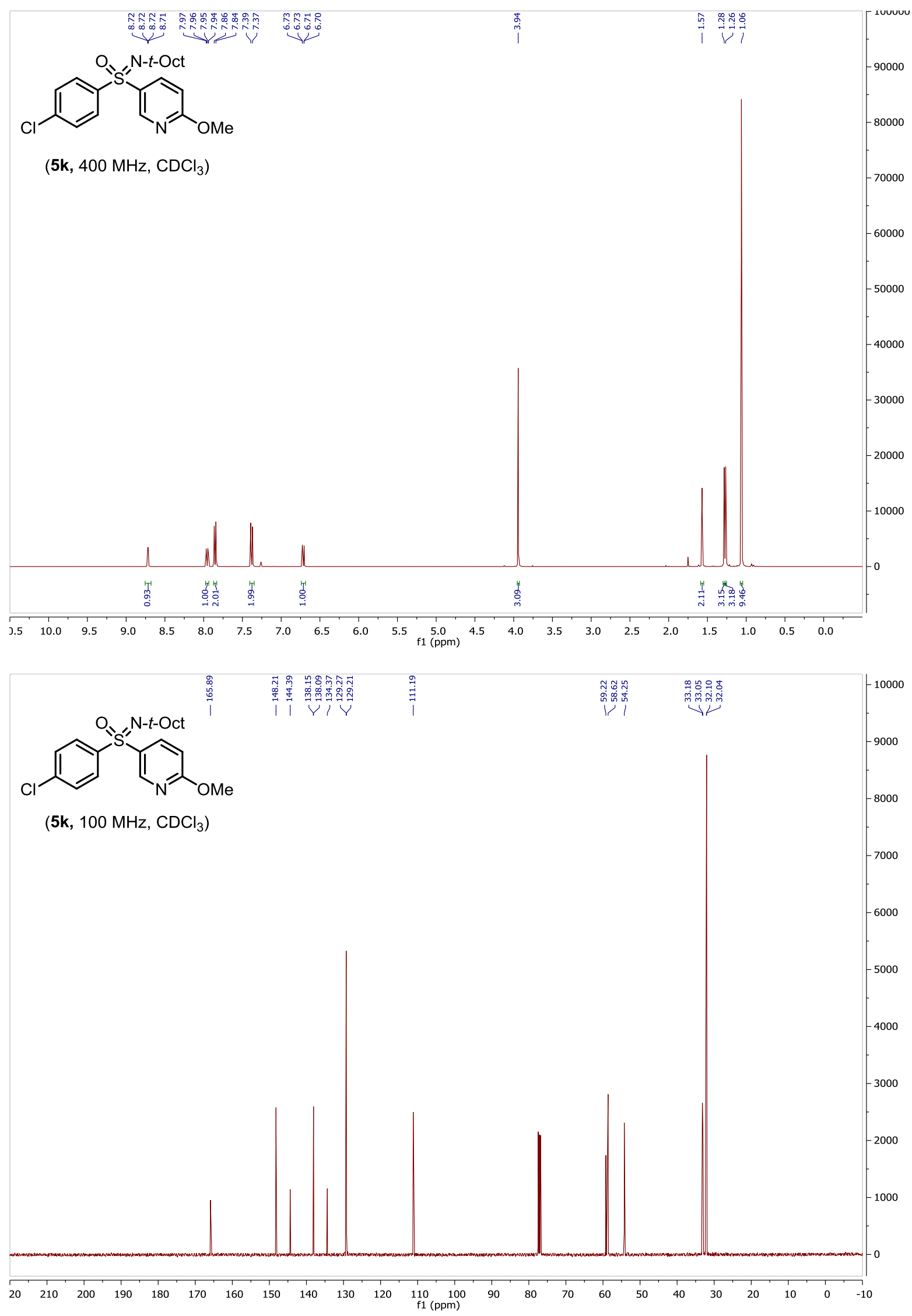

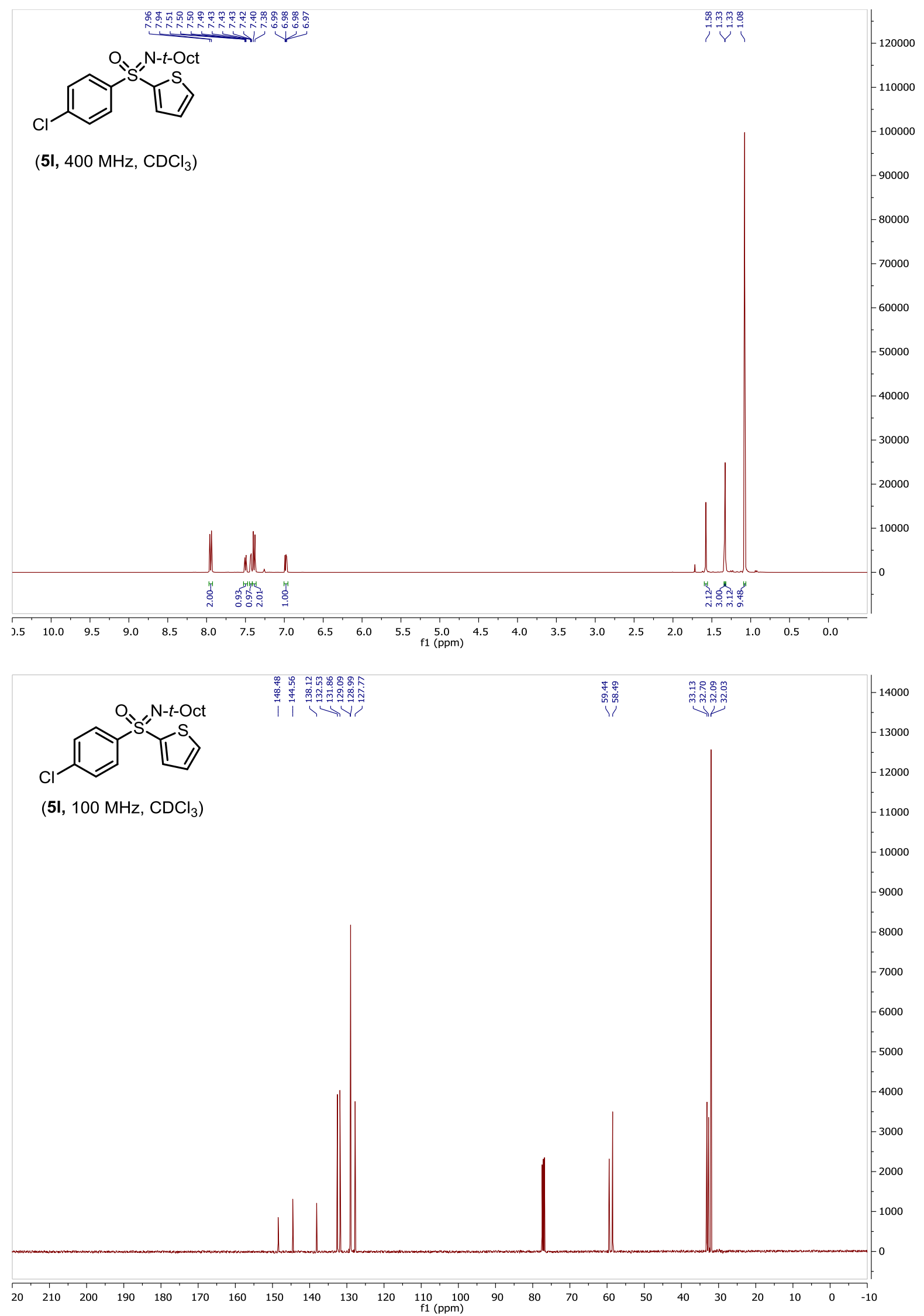


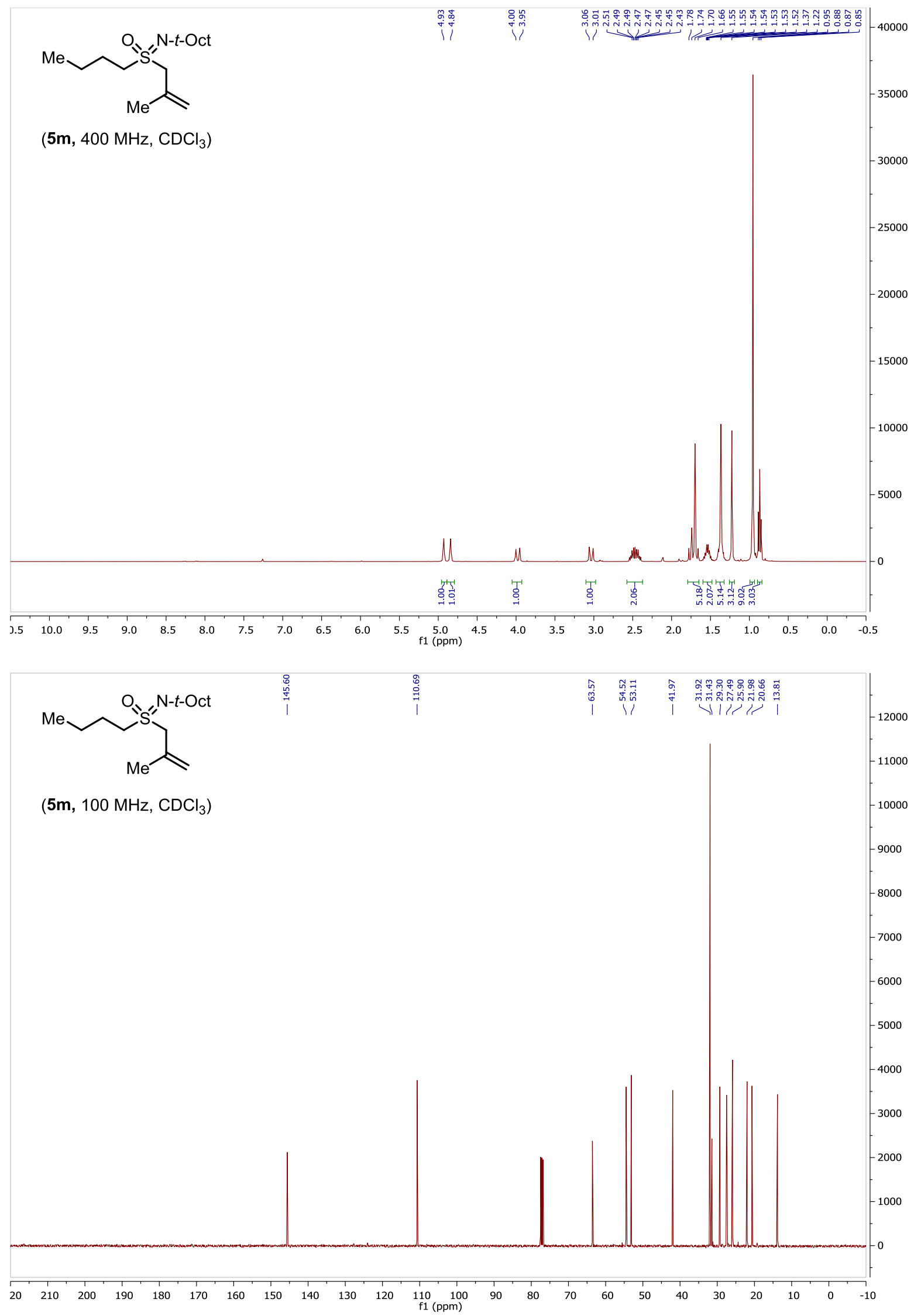




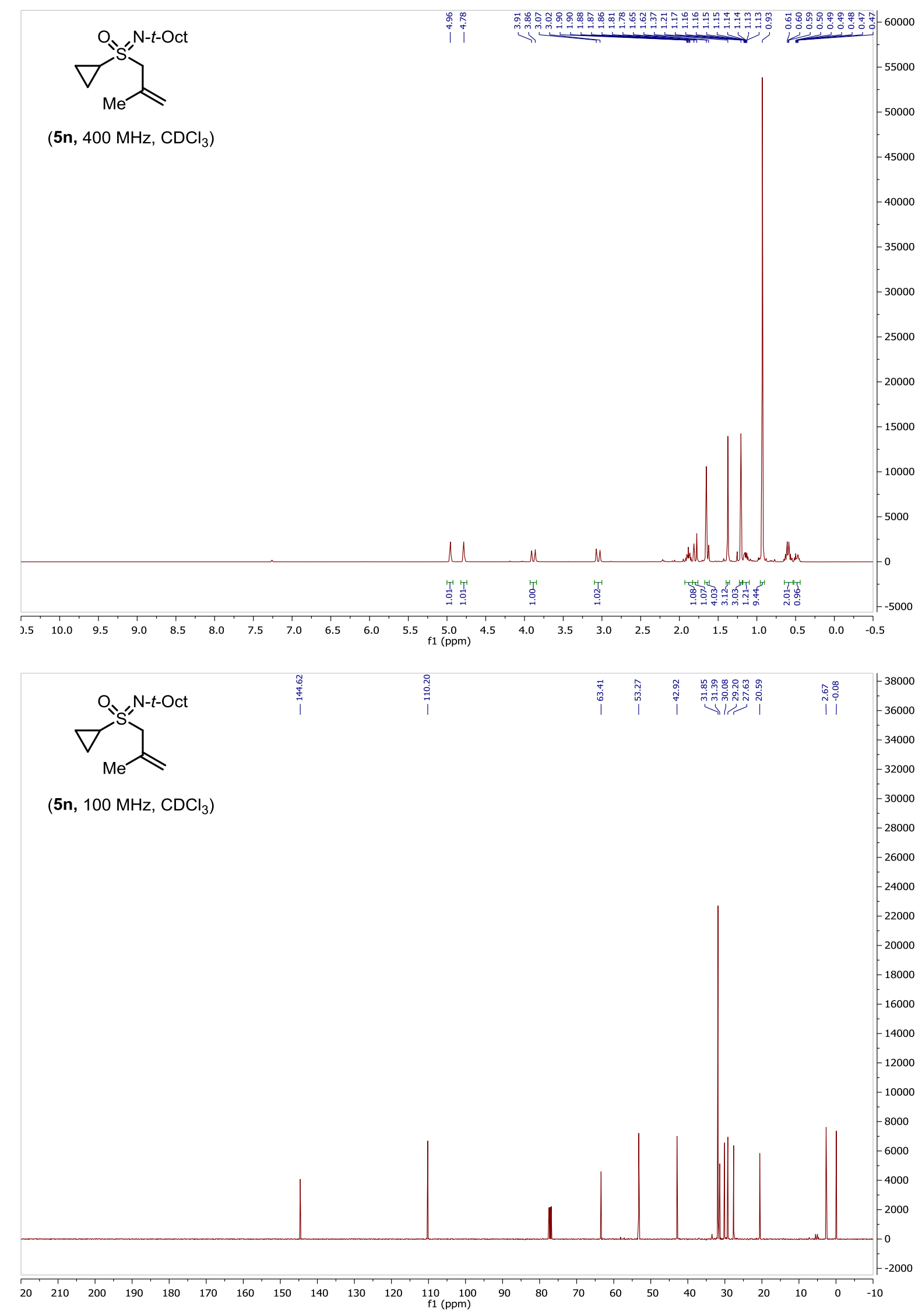




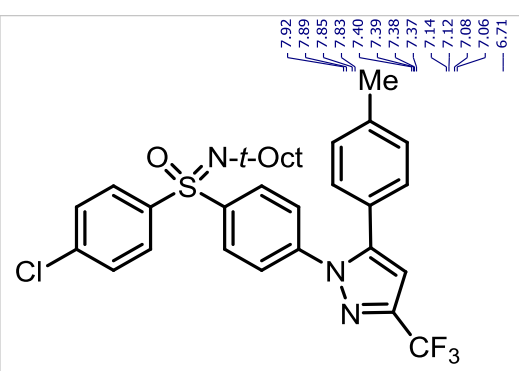

(5o, $400 \mathrm{MHz}, \mathrm{CDCl}_{3}$ )
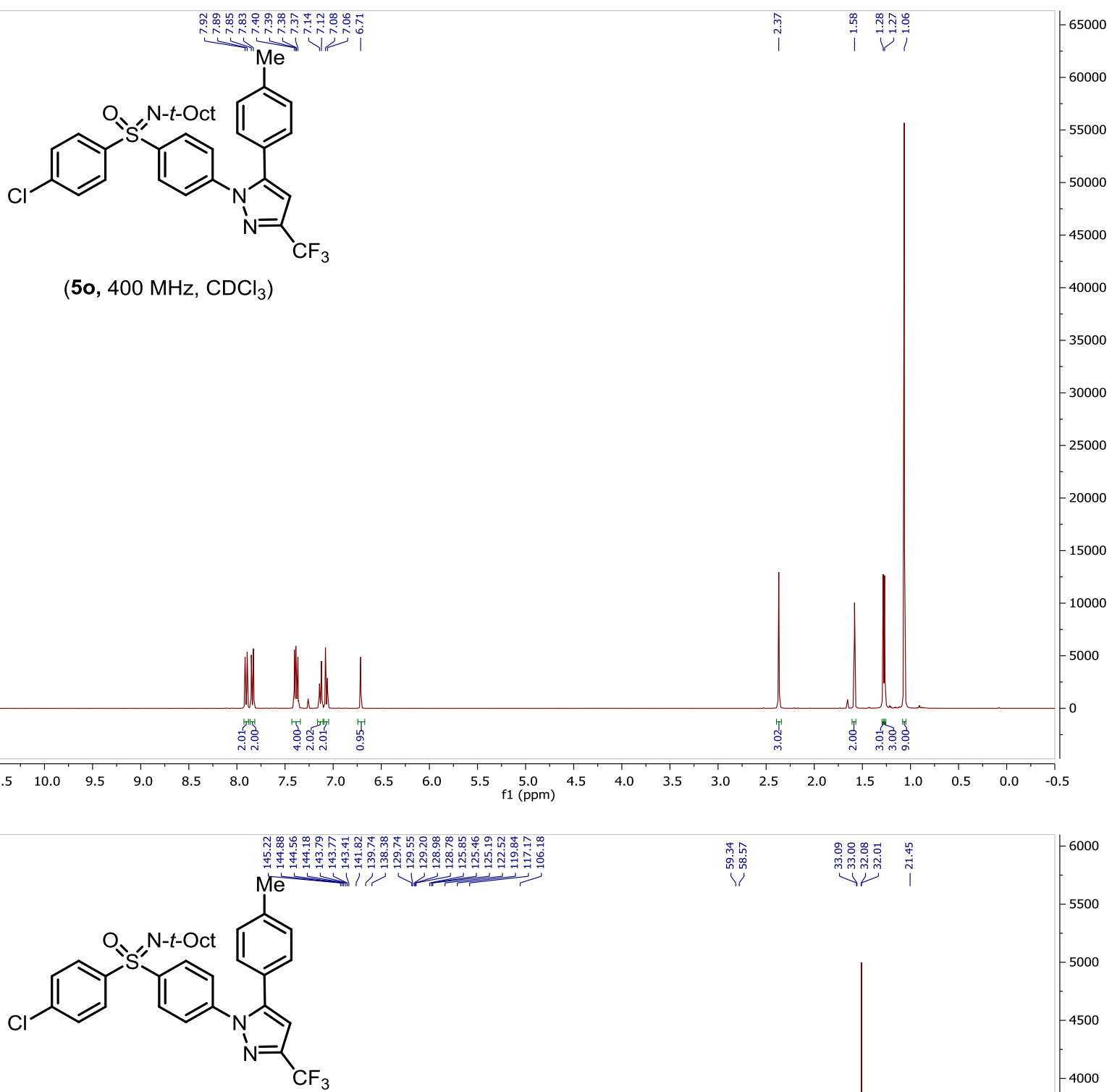

(5o, $100 \mathrm{MHz}, \mathrm{CDCl}_{3}$ )
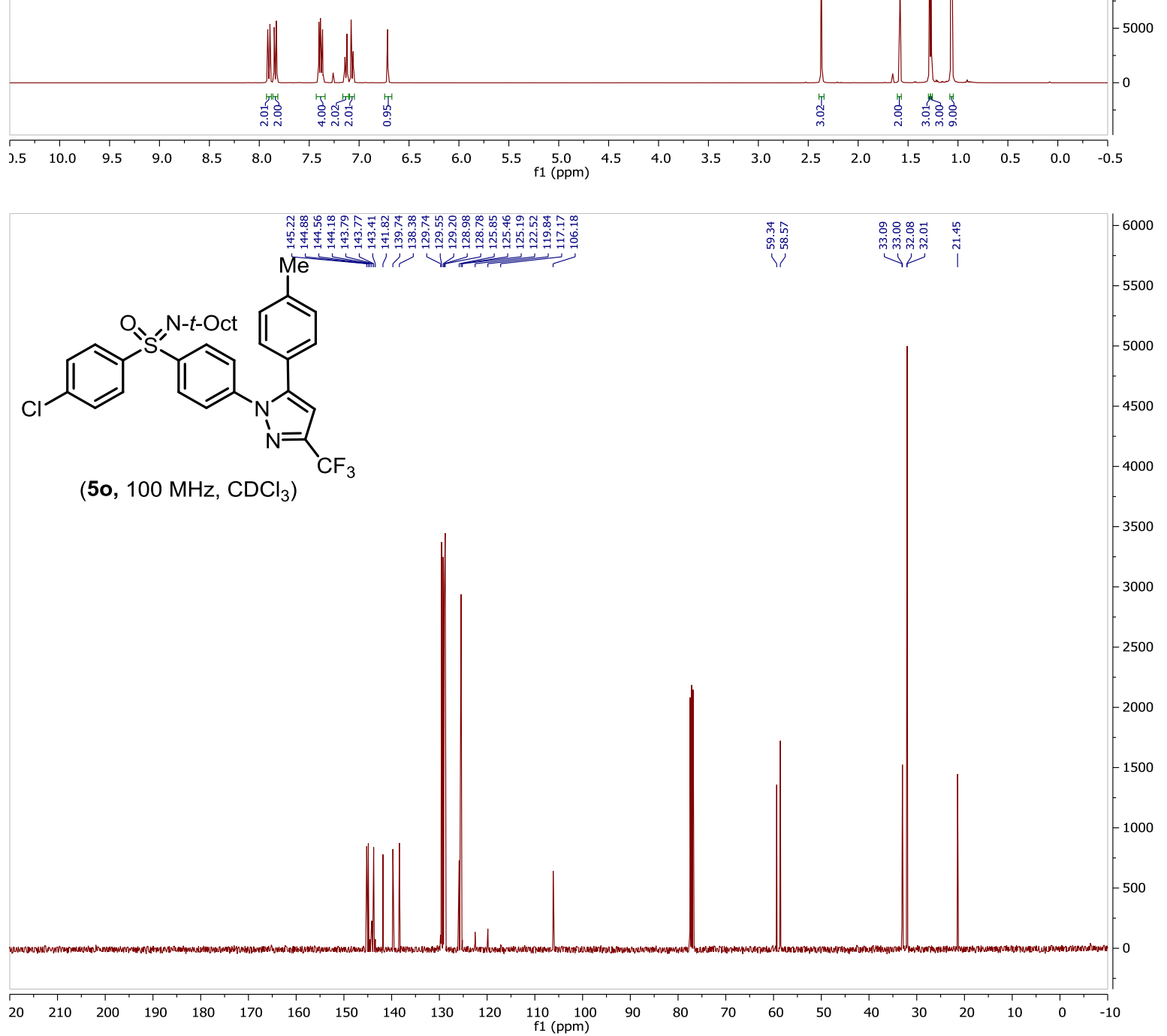


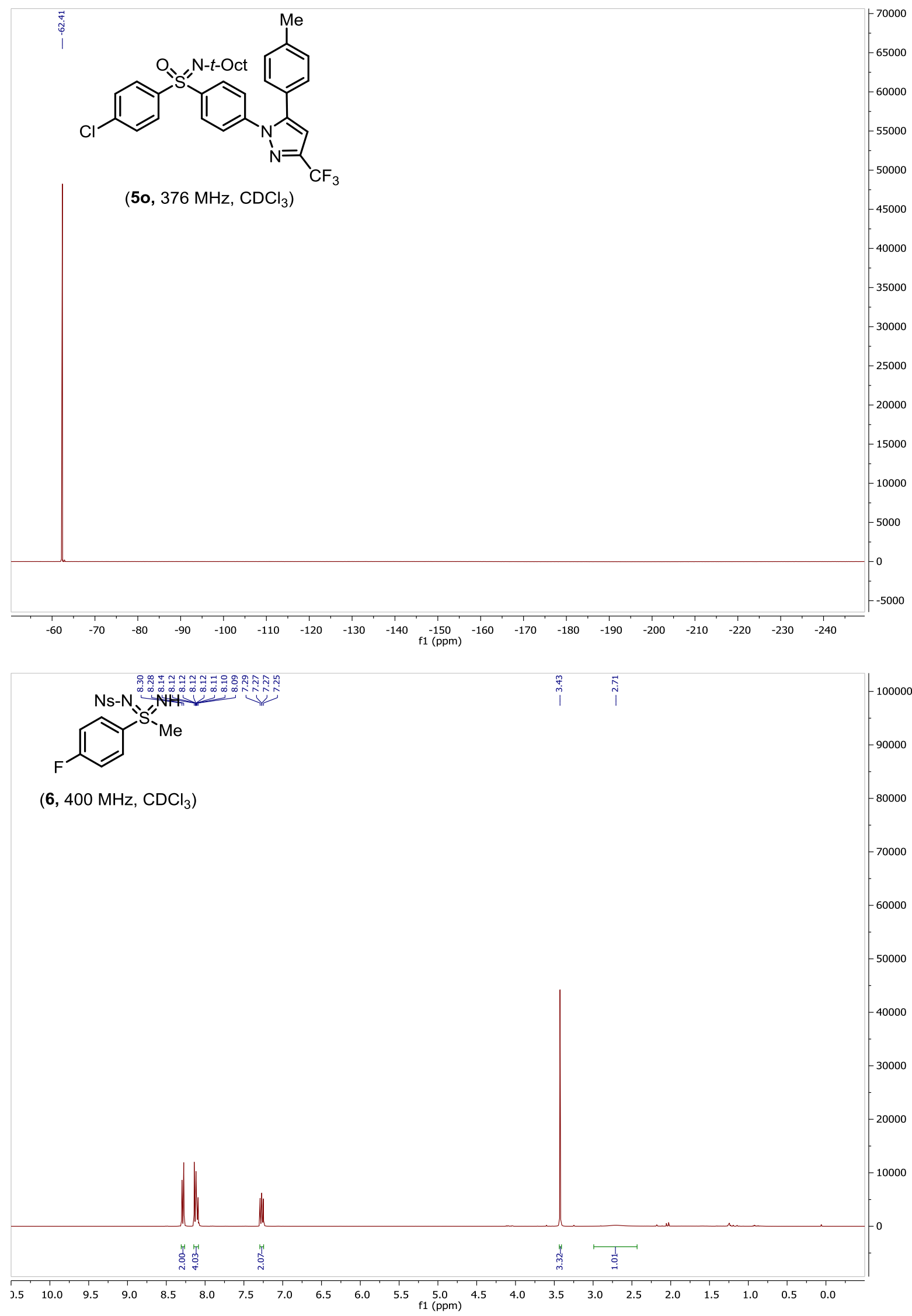



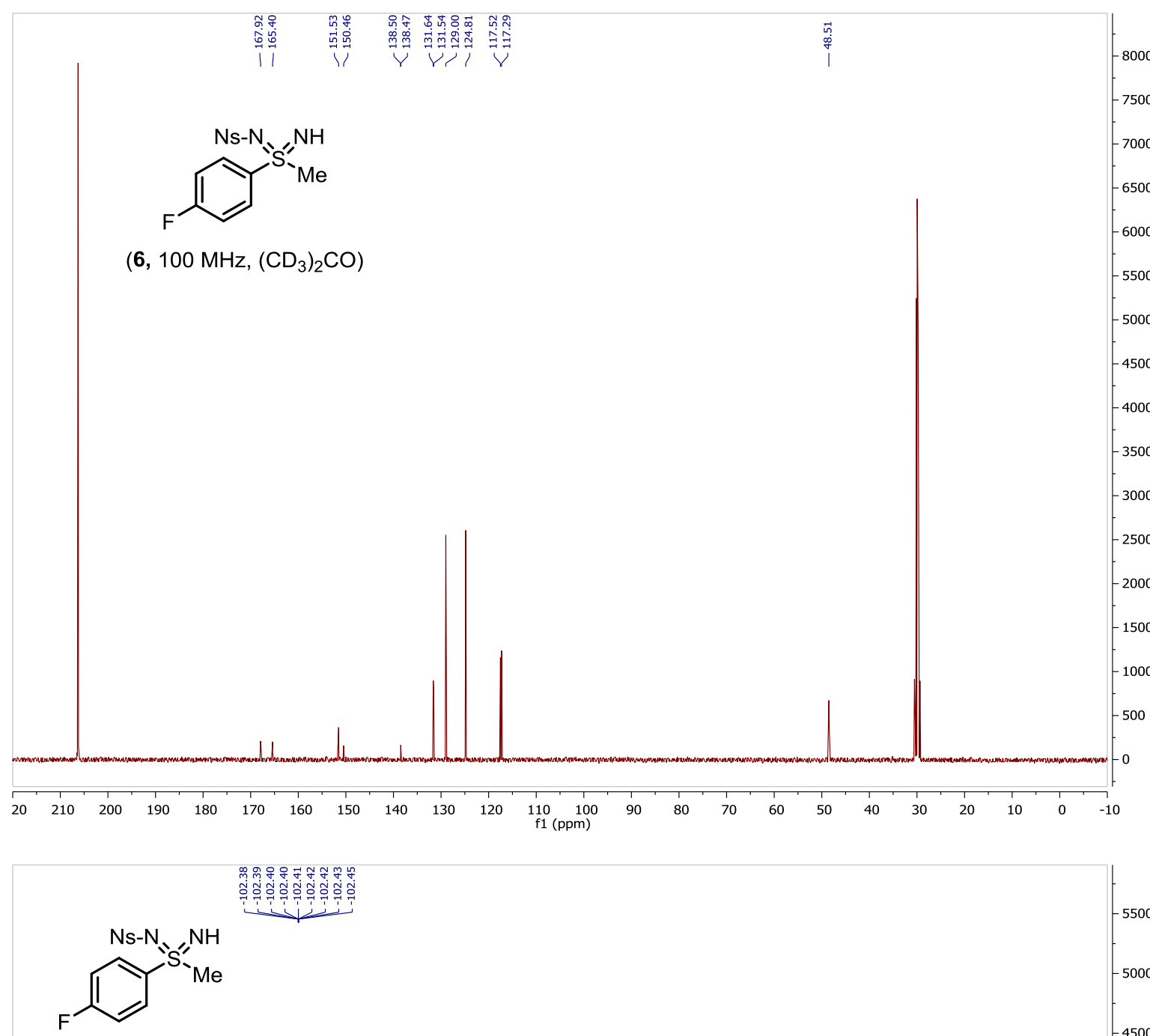

(6, $376 \mathrm{MHz}, \mathrm{CDCl}_{3}$ )

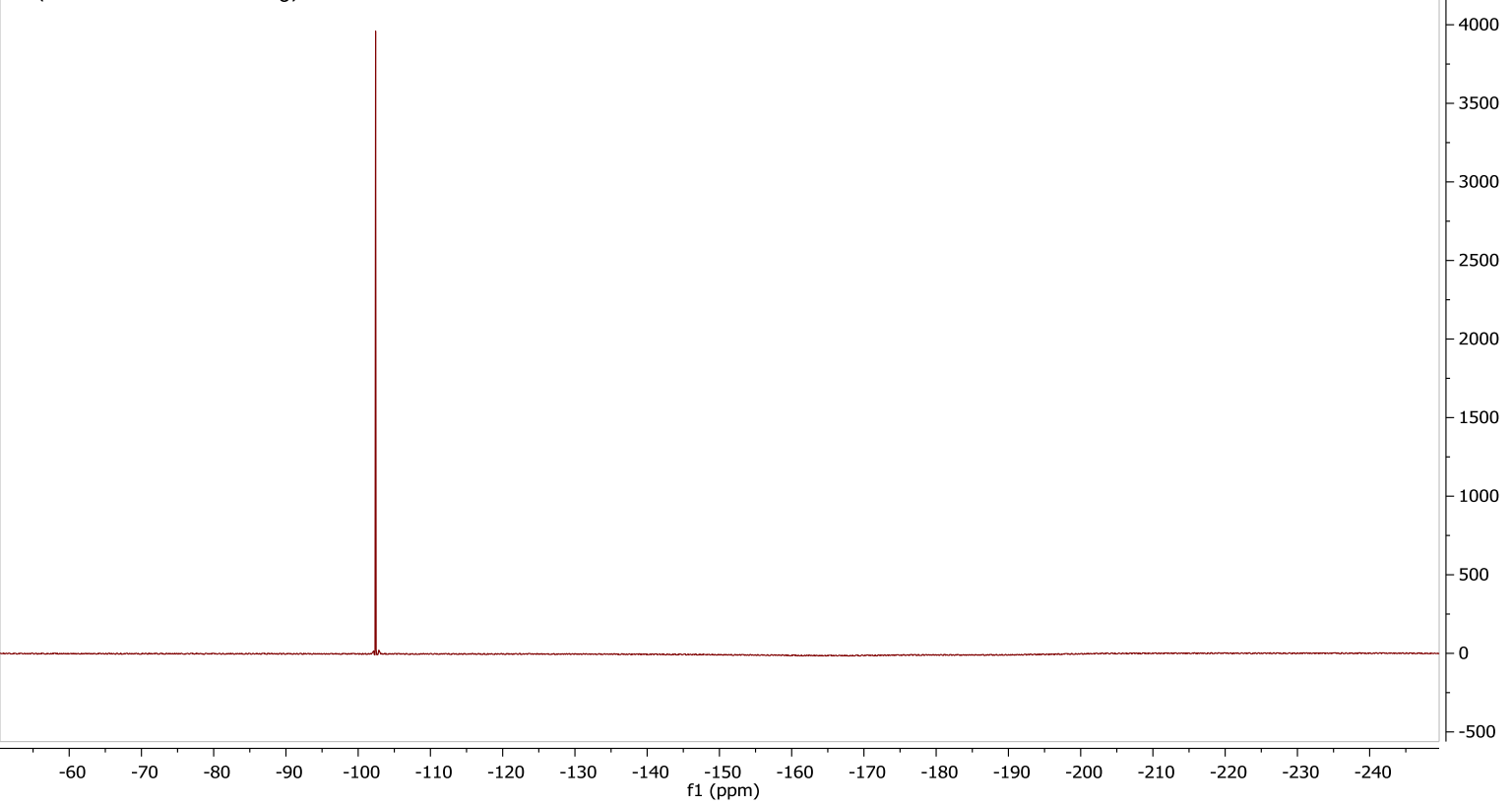



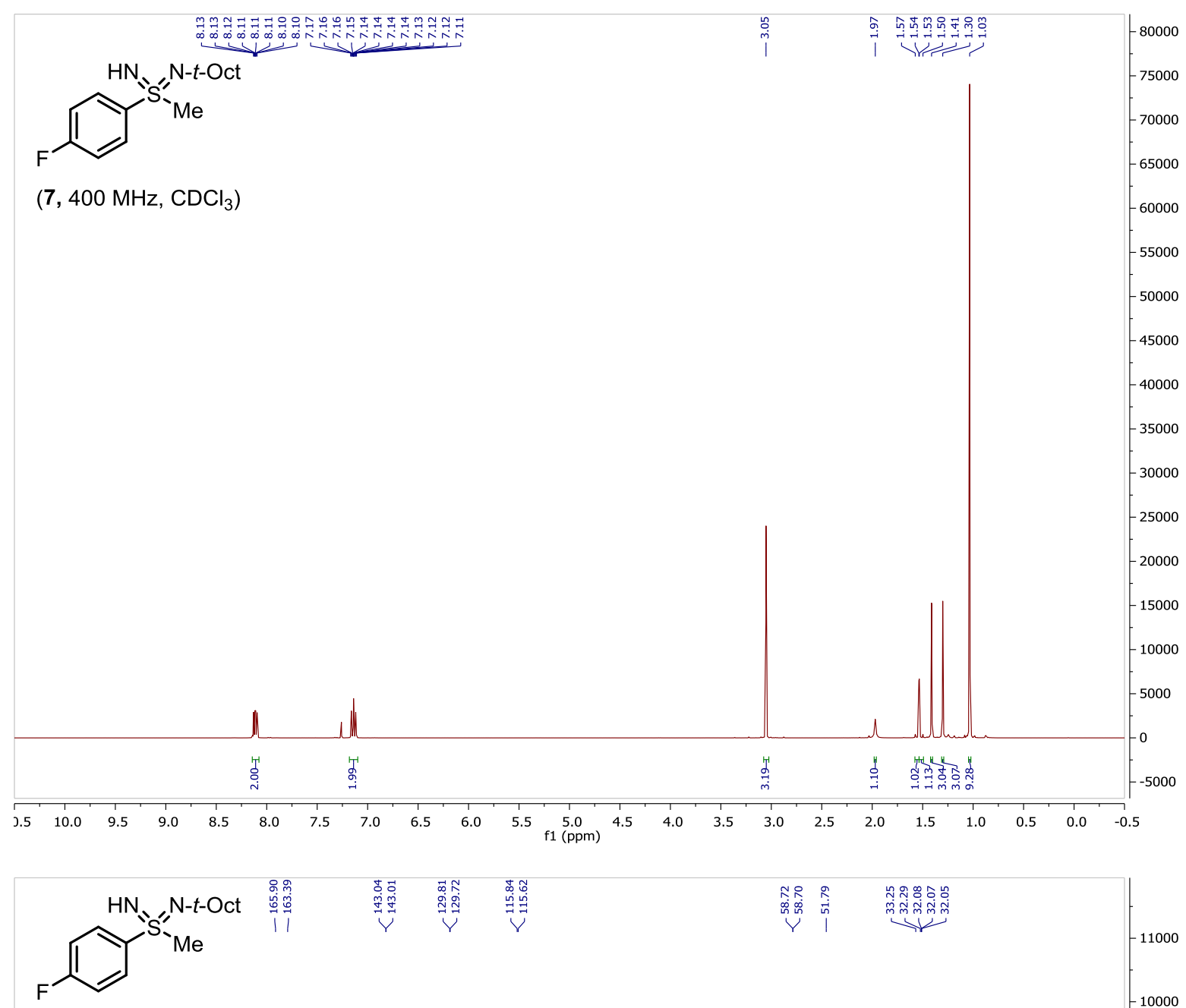

(7, $\left.100 \mathrm{MHz}, \mathrm{CDCl}_{3}\right)$

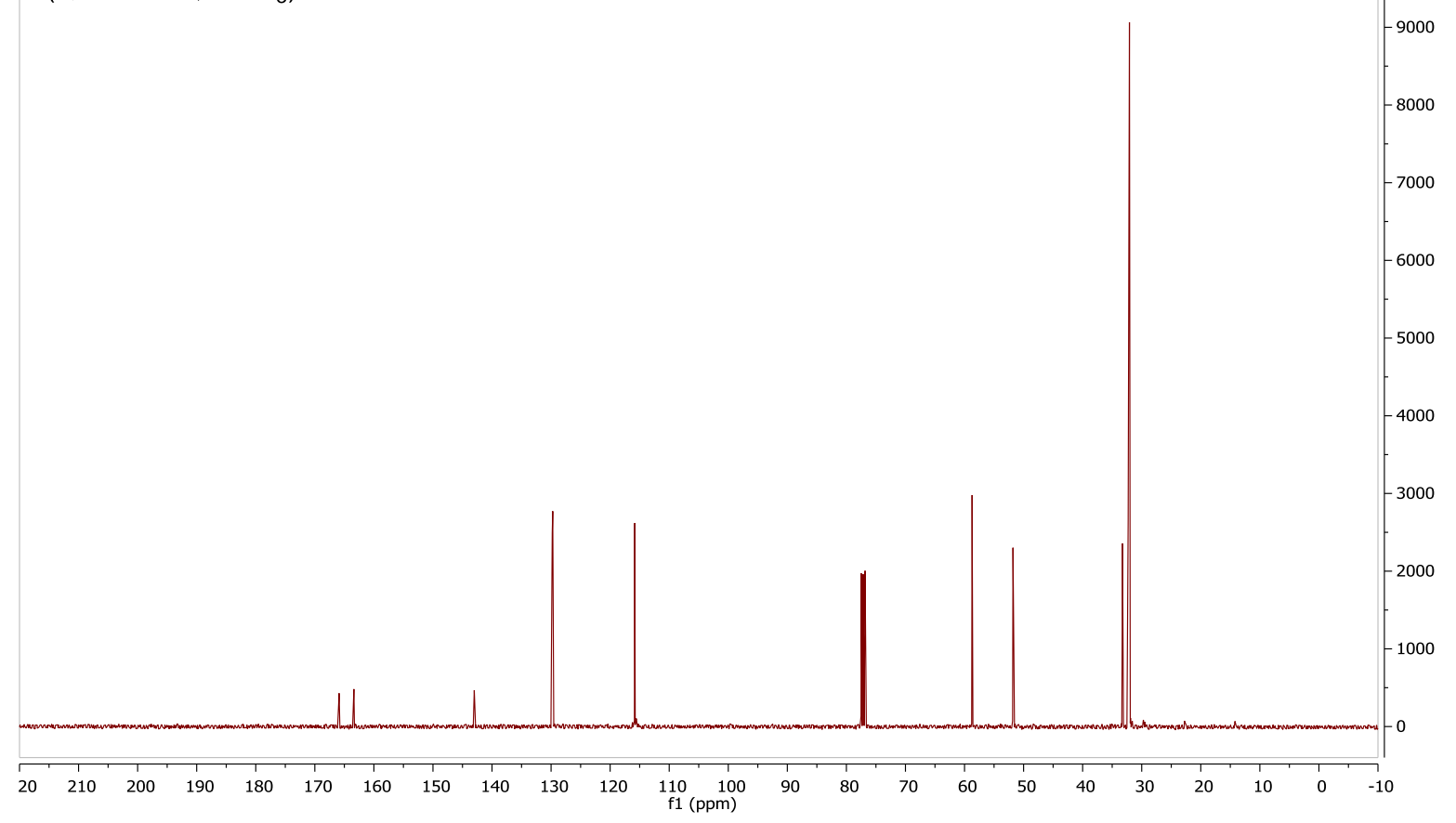



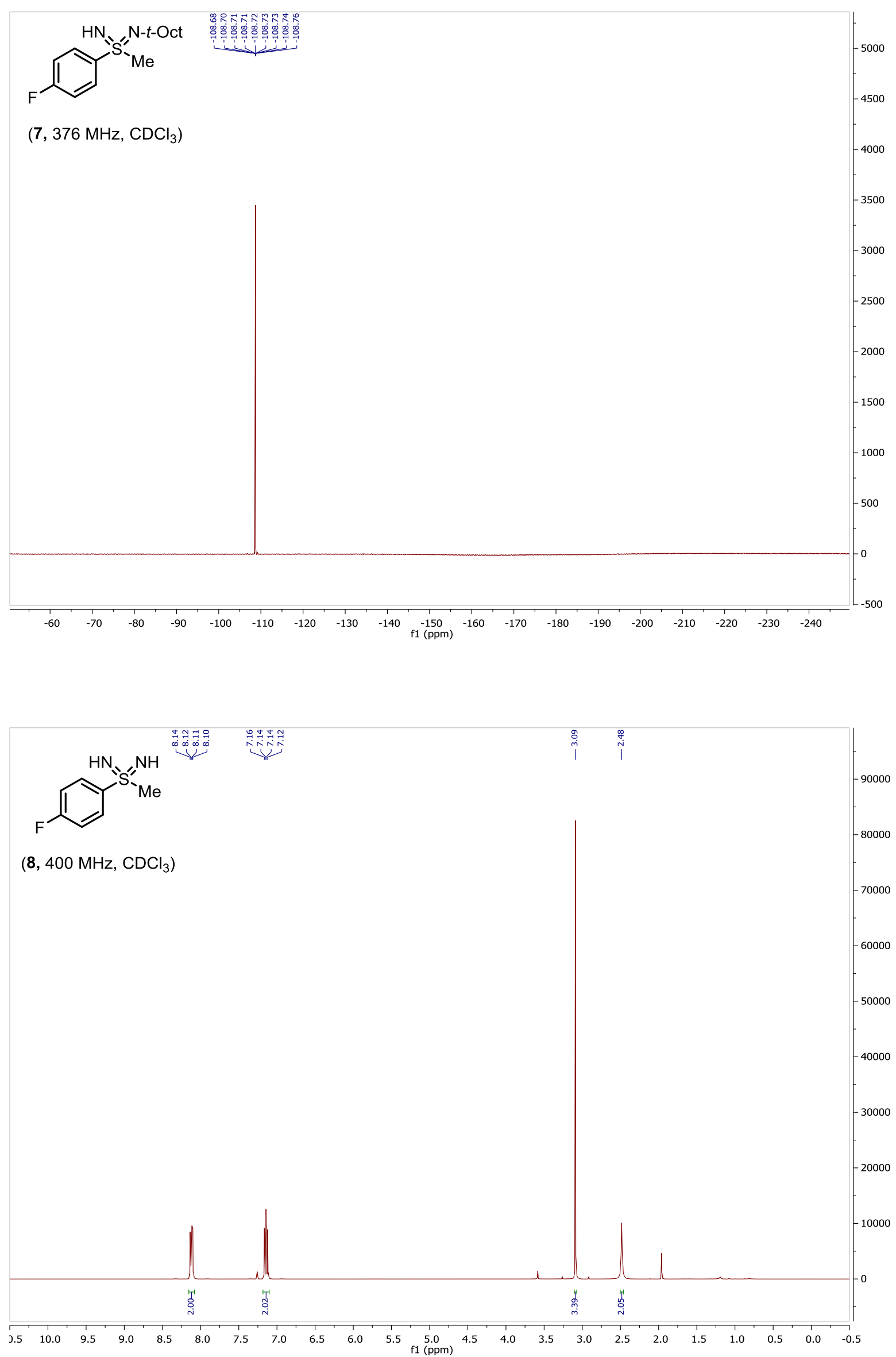

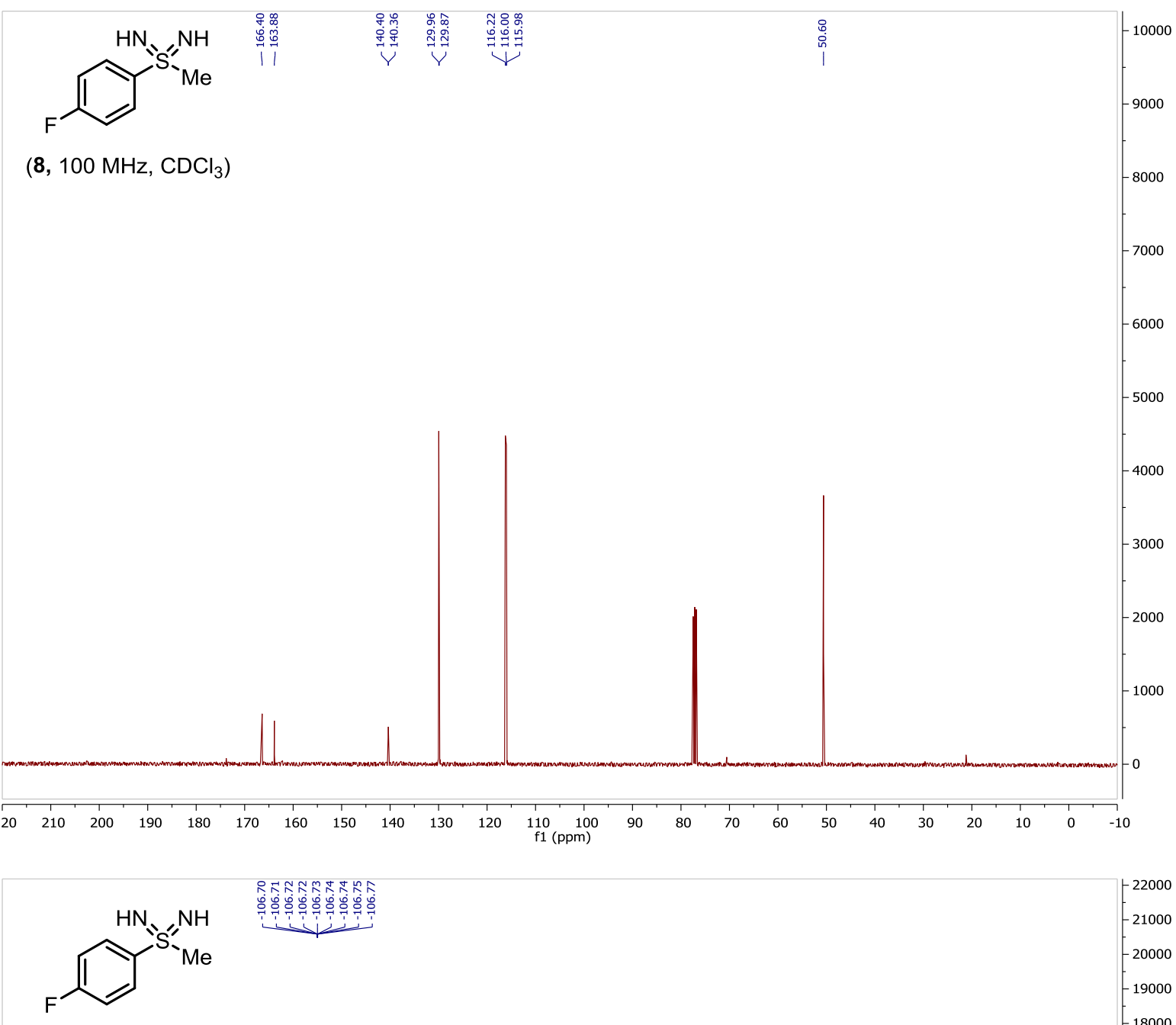

(8, $\left.376 \mathrm{MHz}, \mathrm{CDCl}_{3}\right)$

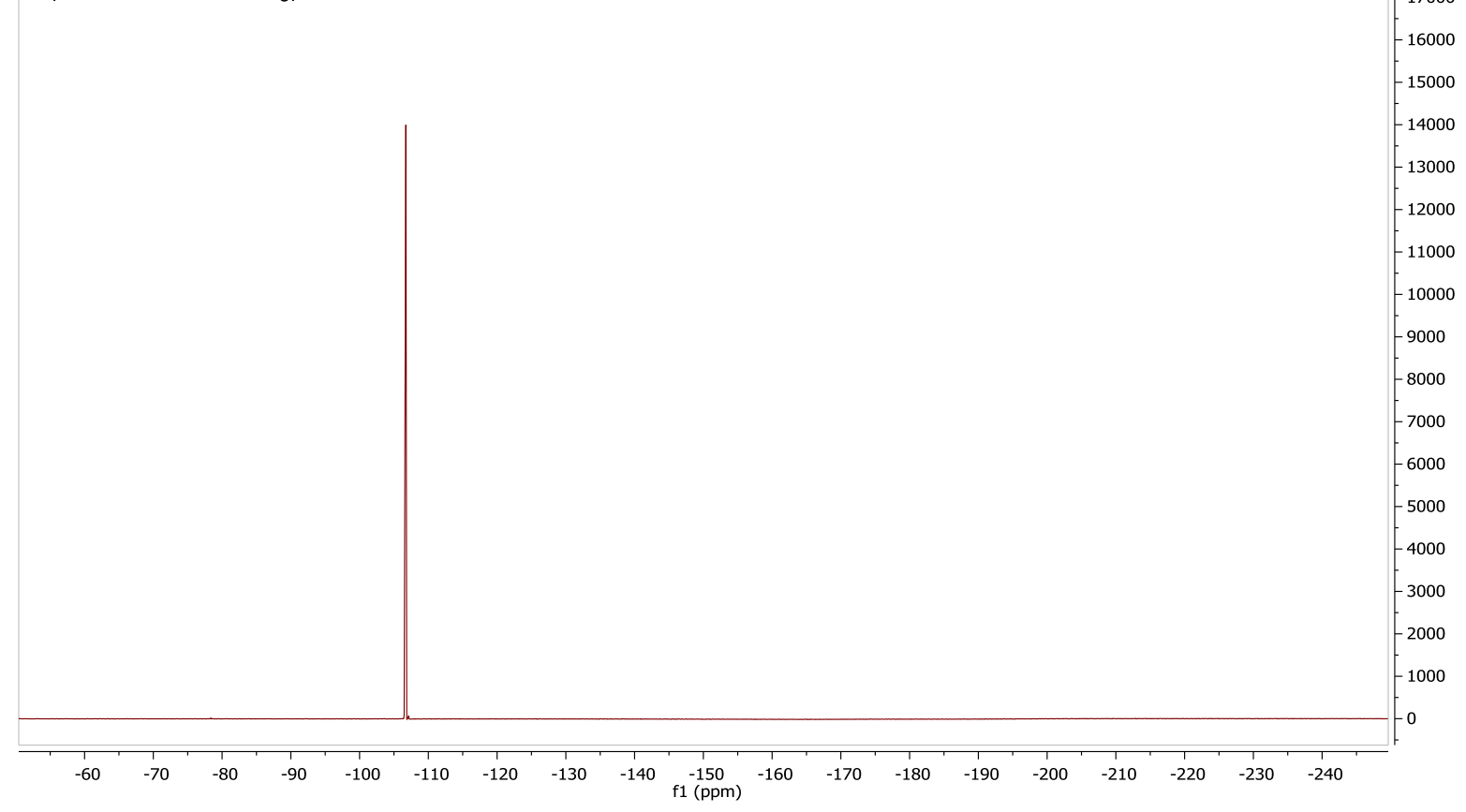



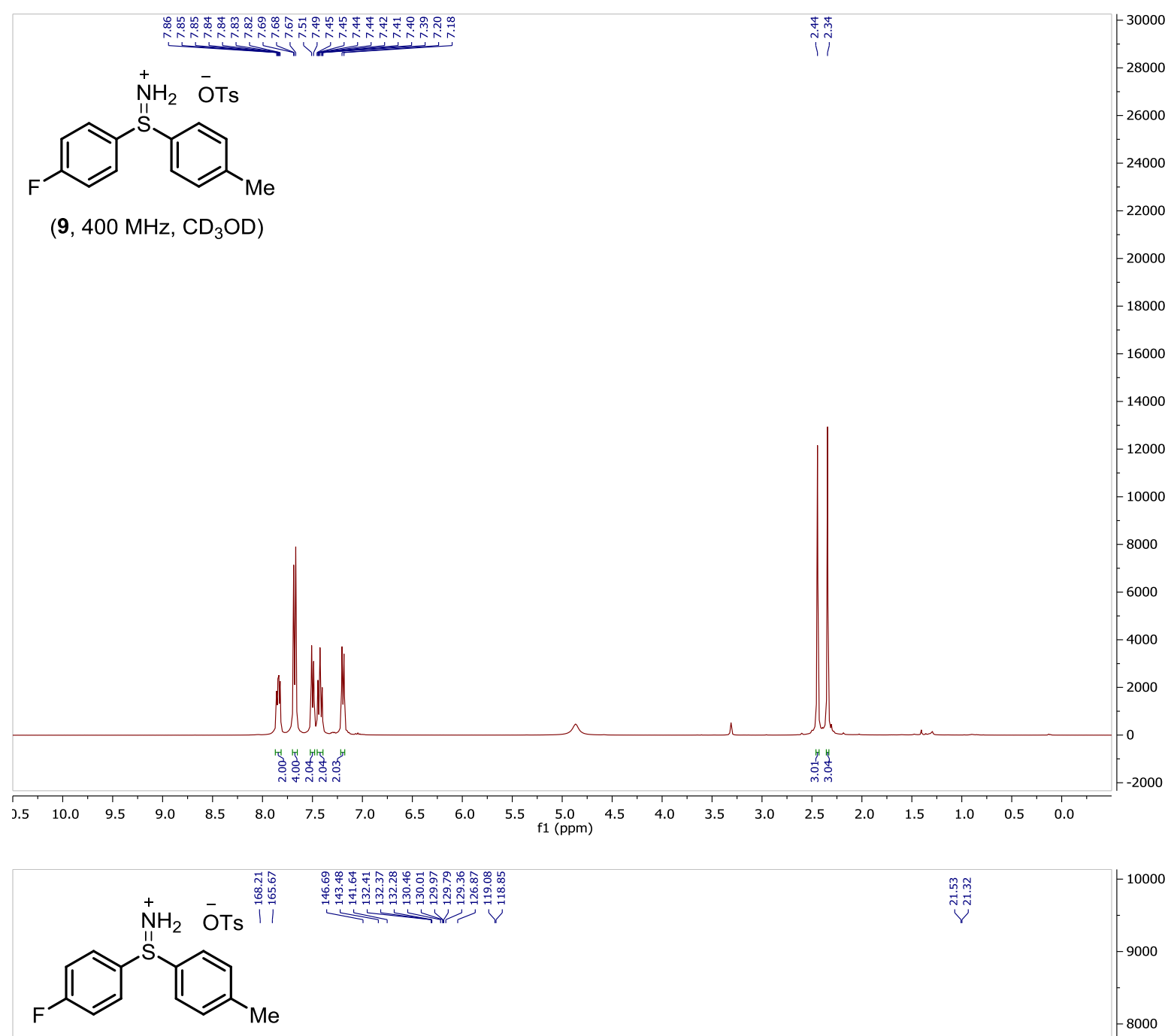

(9, $\left.100 \mathrm{MHz}, \mathrm{CD}_{3} \mathrm{OD}\right)$

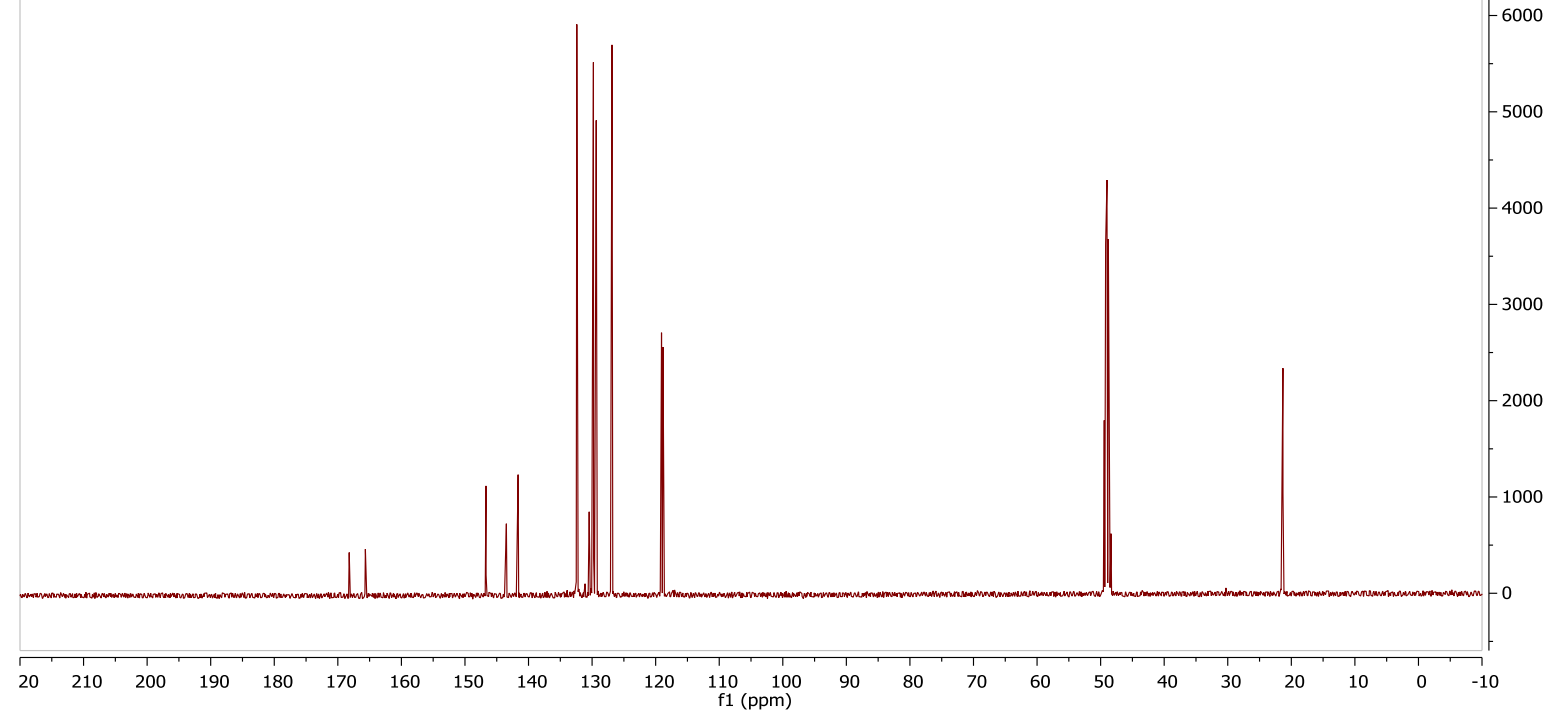




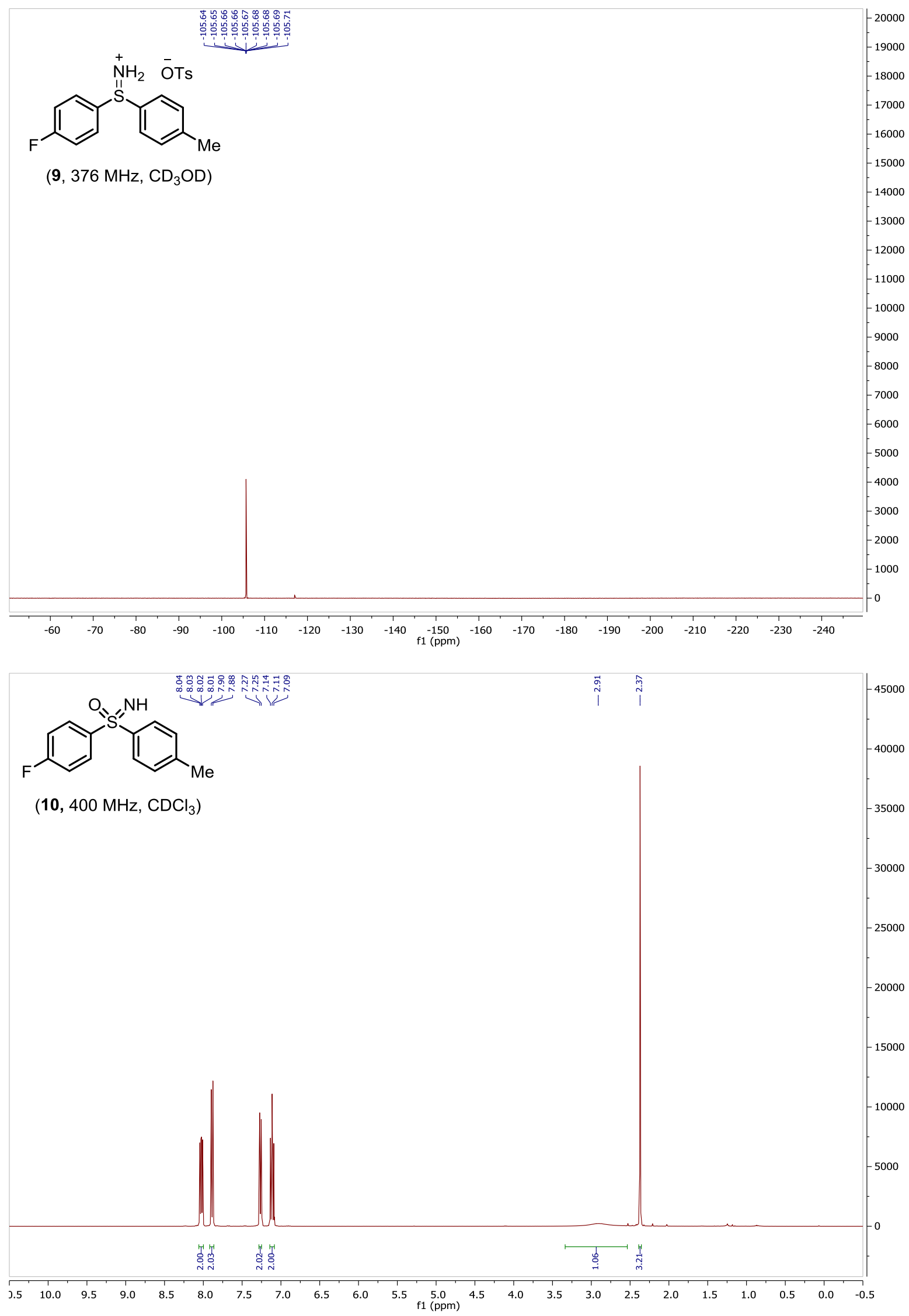



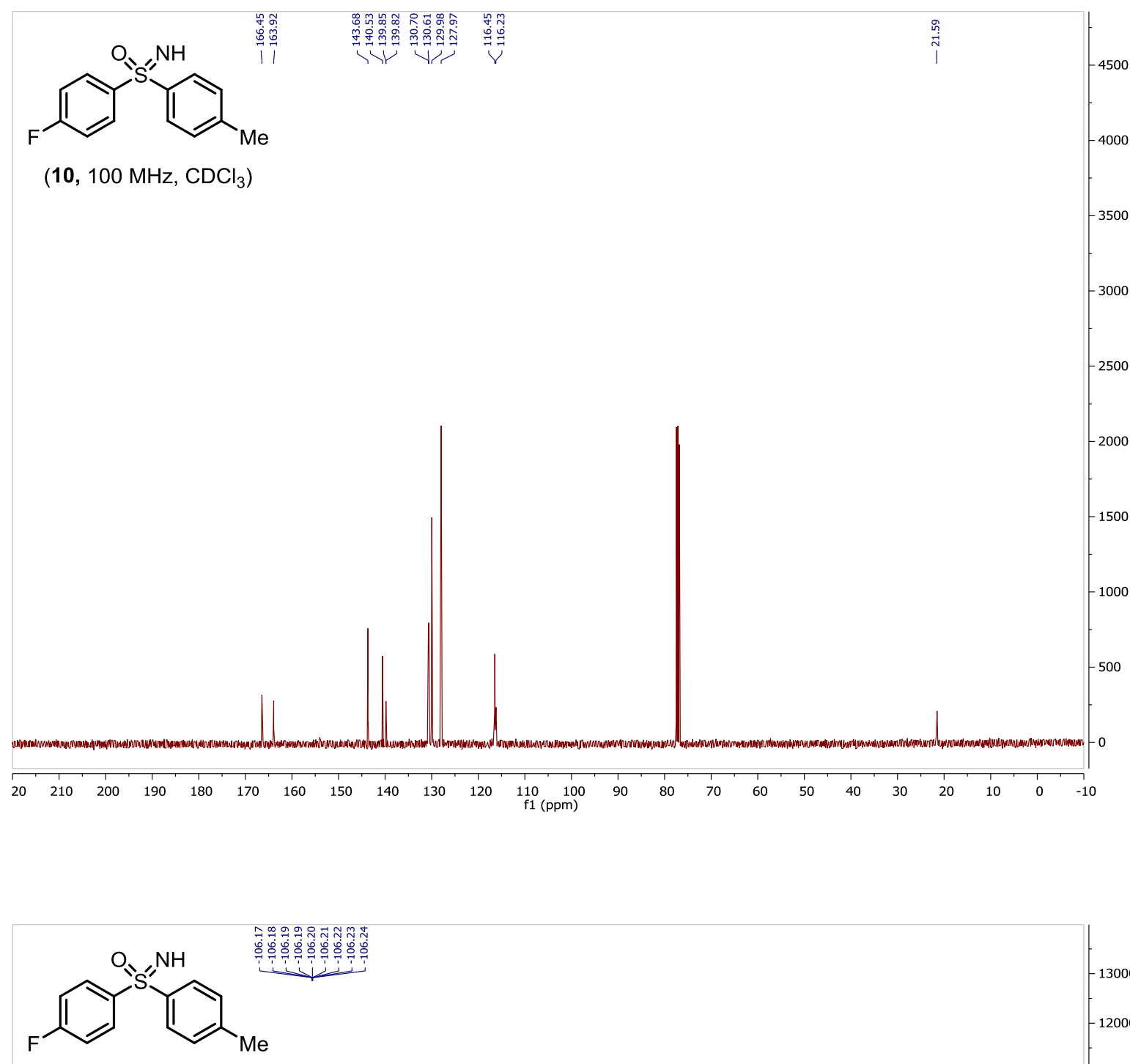

$\left(10,376 \mathrm{MHz}, \mathrm{CDCl}_{3}\right)$

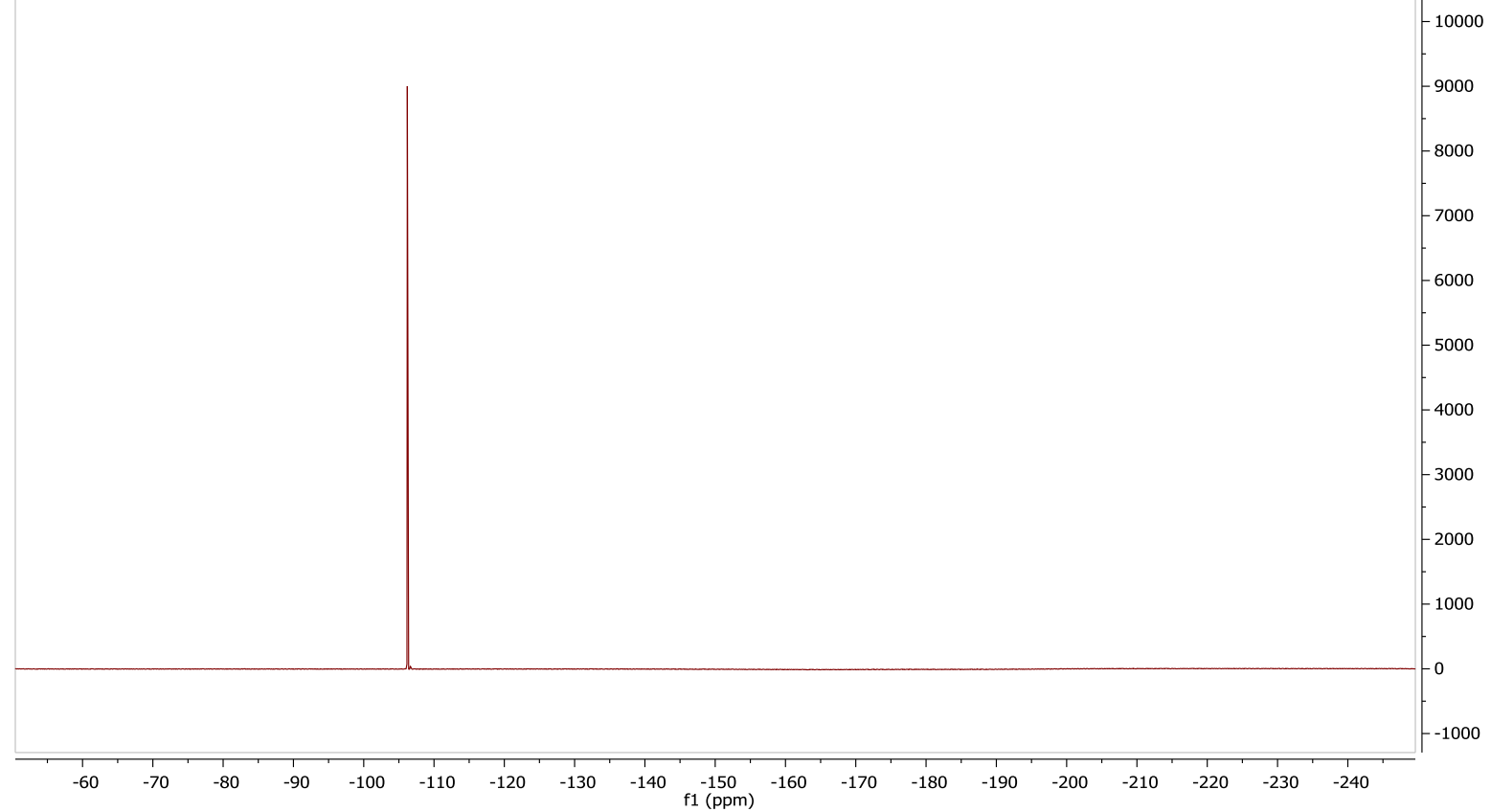




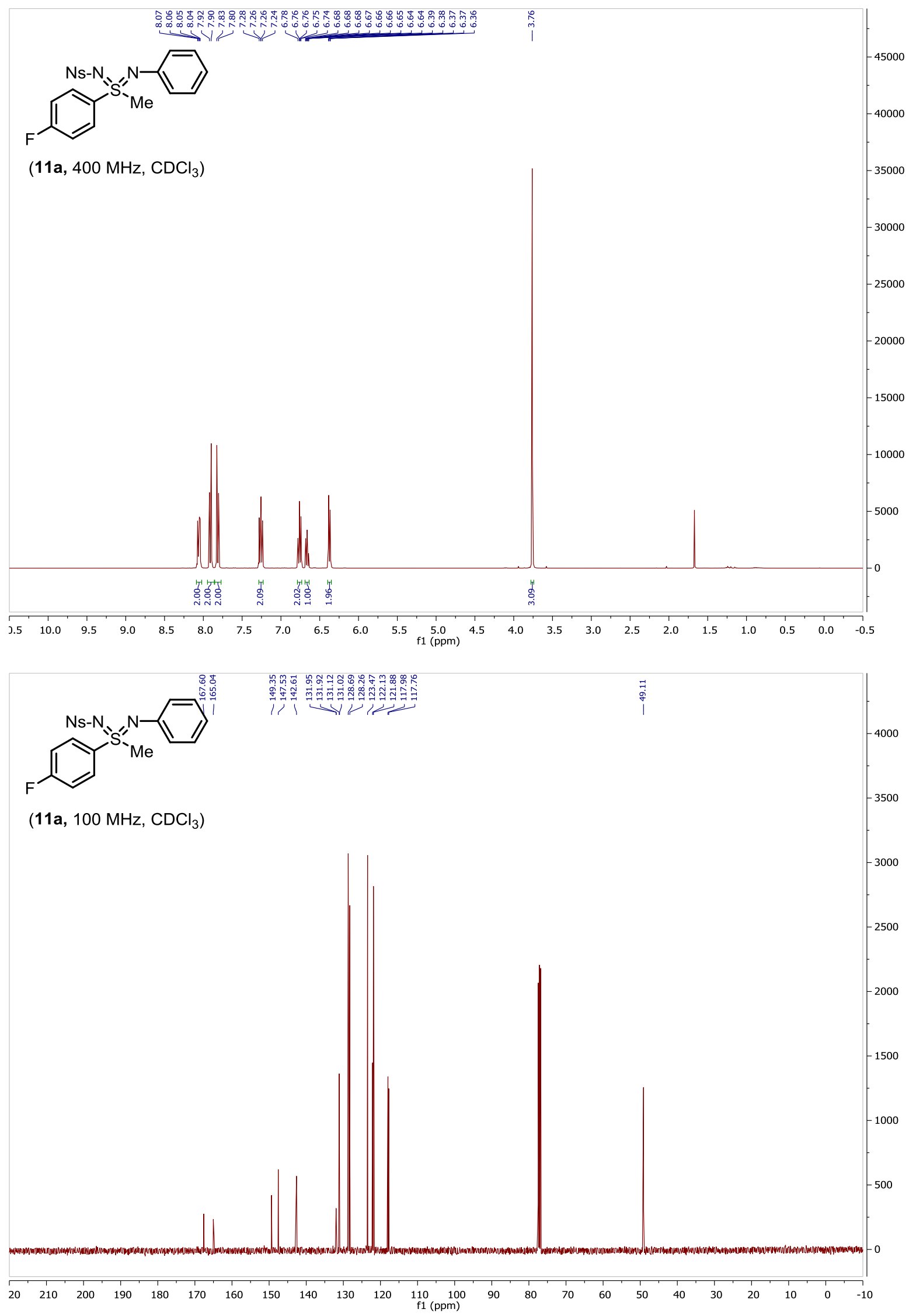




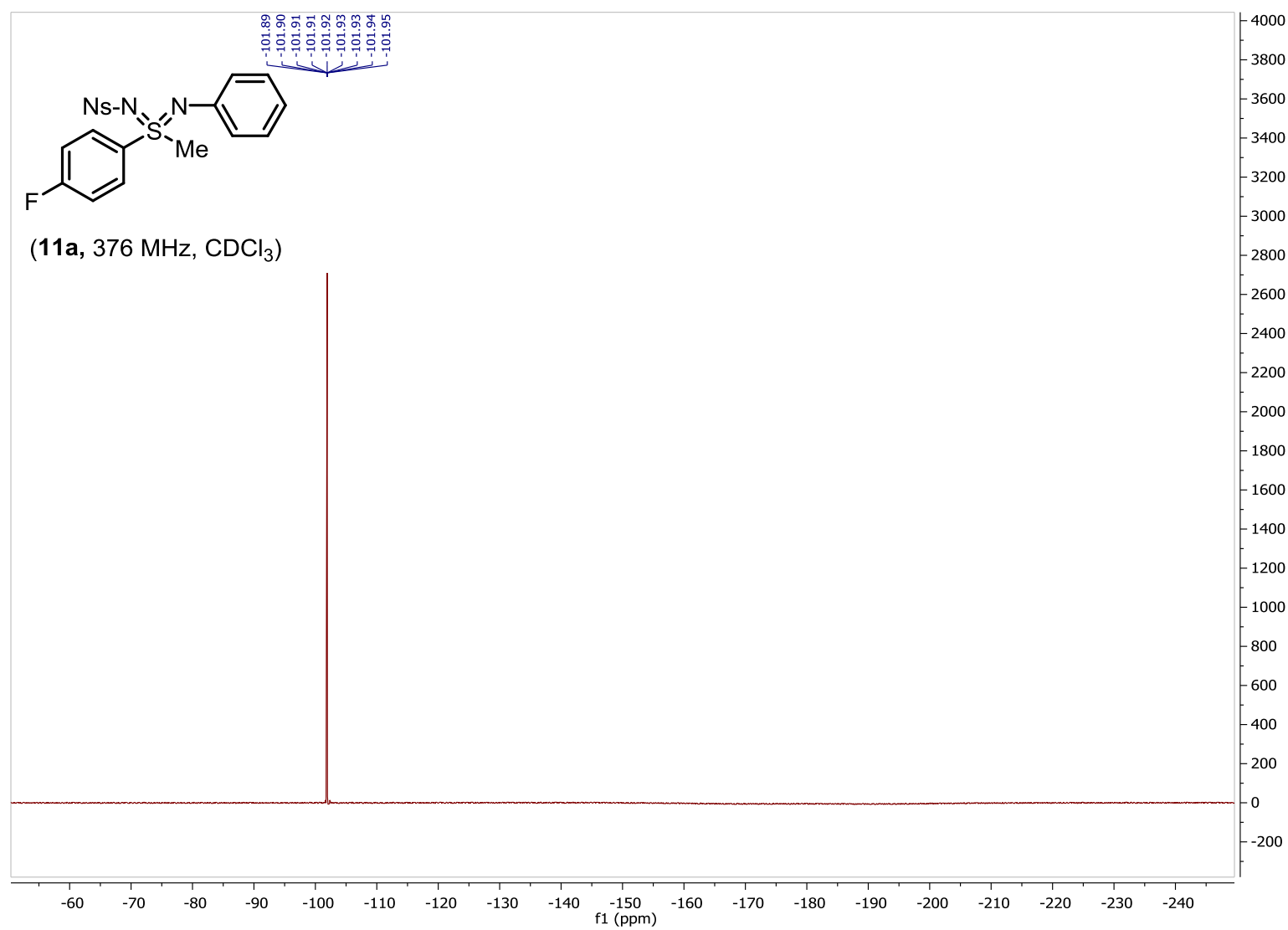

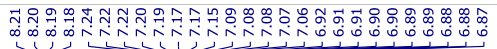

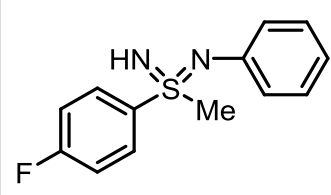

$\left(11,400 \mathrm{MHz}, \mathrm{CDCl}_{3}\right)$

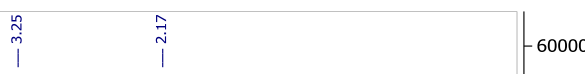

50000

45000

40000

$-35000$

$-30000$

25000

$-20000$

5000

$-10000$

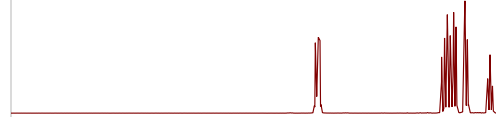

$-5000$

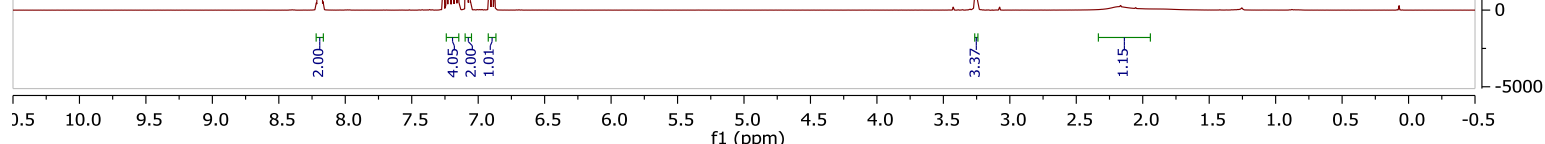




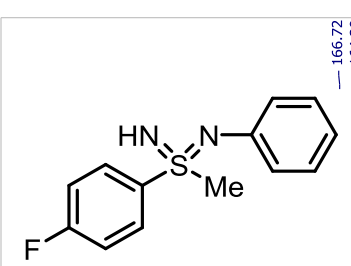

$\overrightarrow{0}$
o.
0

$\left(11,100 \mathrm{MHz}, \mathrm{CDCl}_{3}\right)$
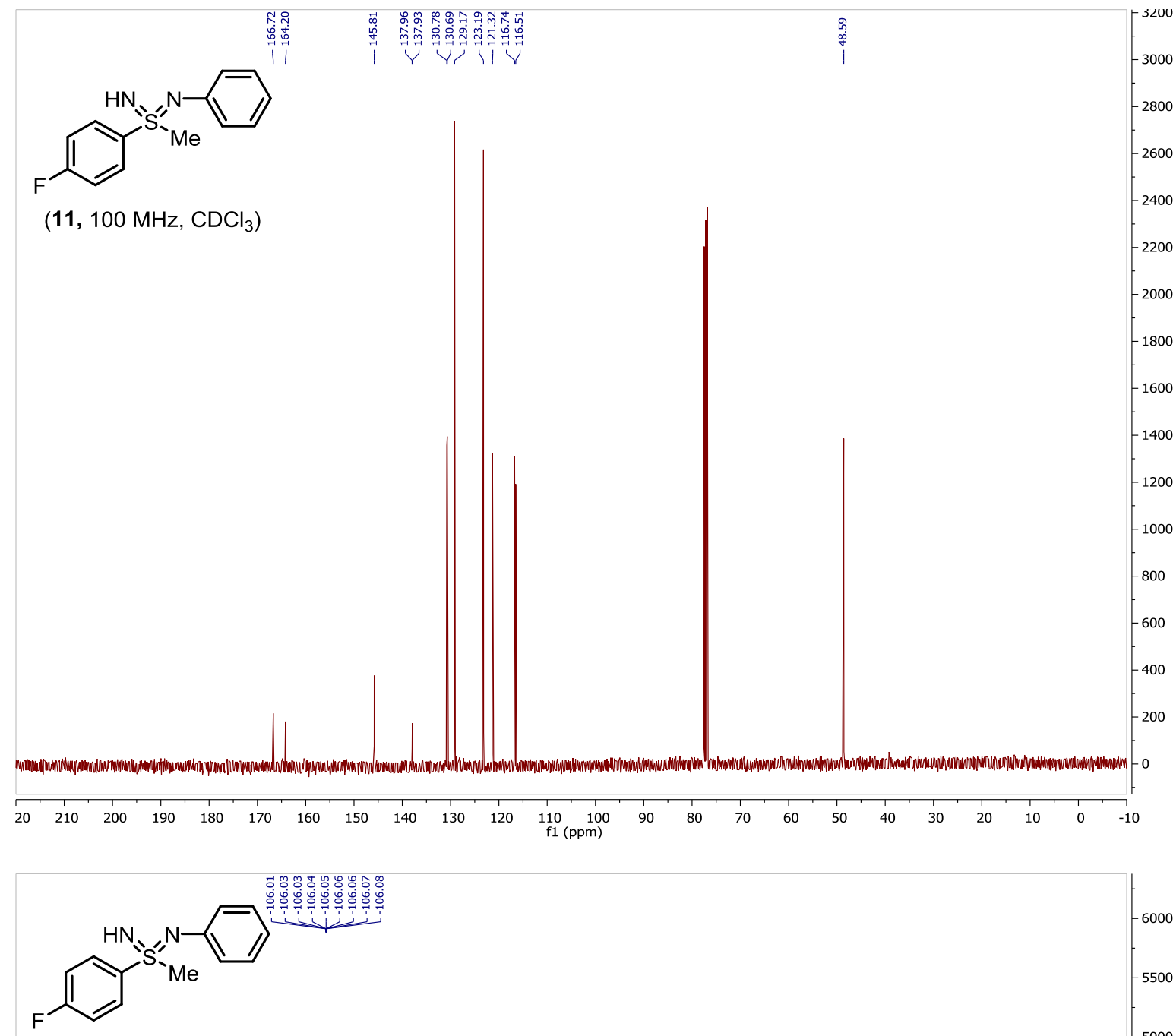

$\left(11,376 \mathrm{MHz}, \mathrm{CDCl}_{3}\right)$

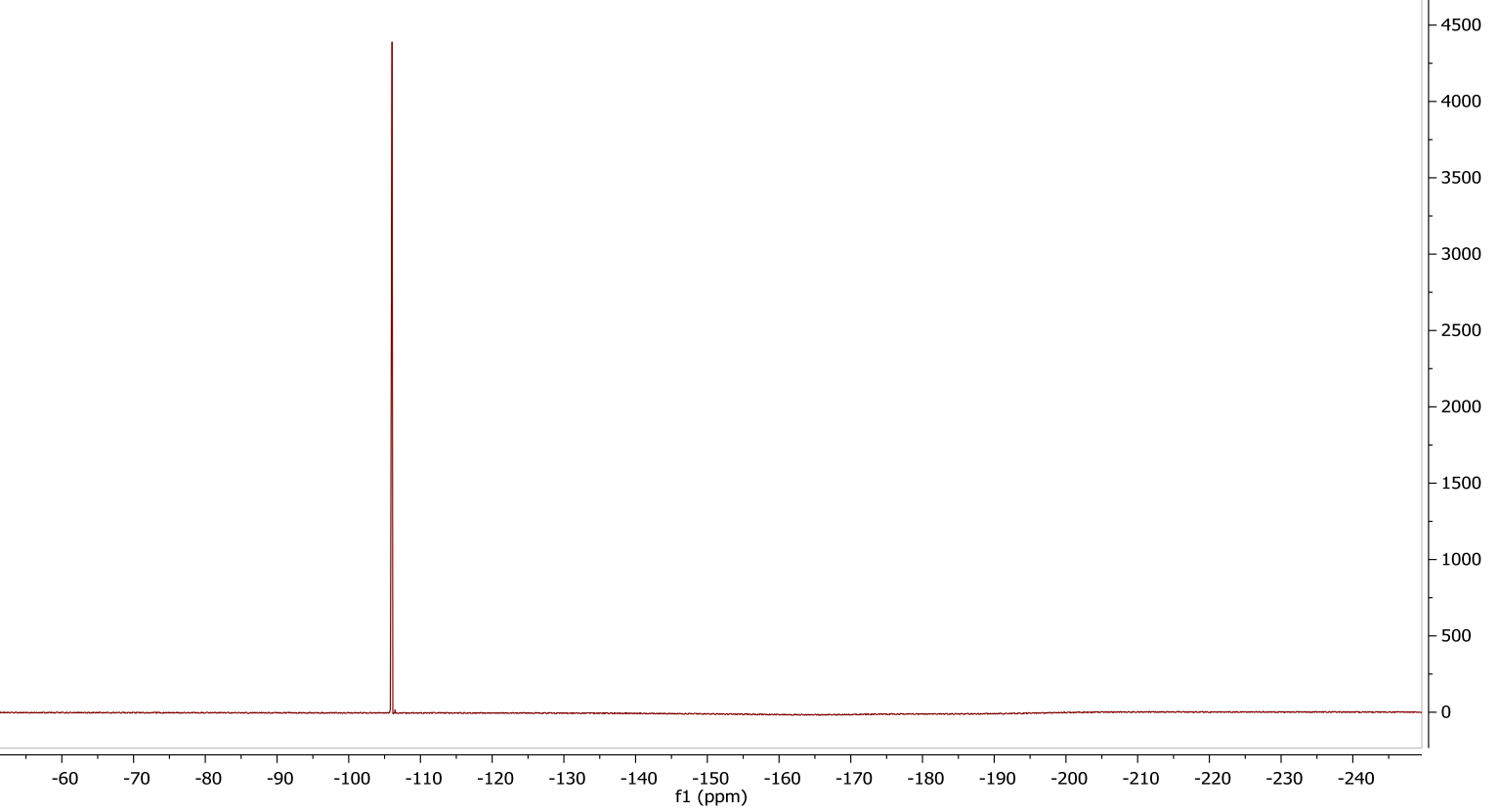



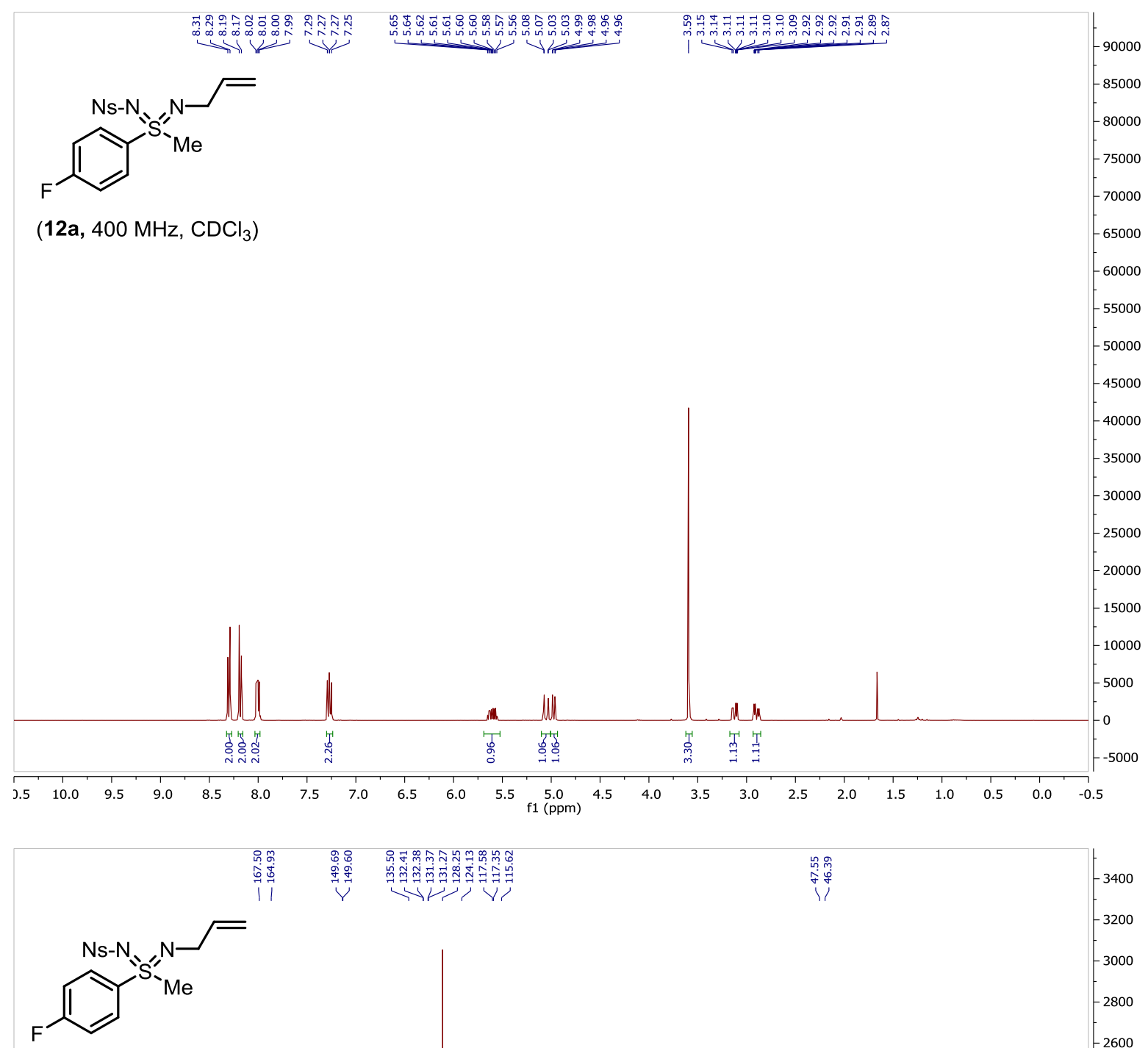

(12a, $100 \mathrm{MHz}, \mathrm{CDCl}_{3}$ )

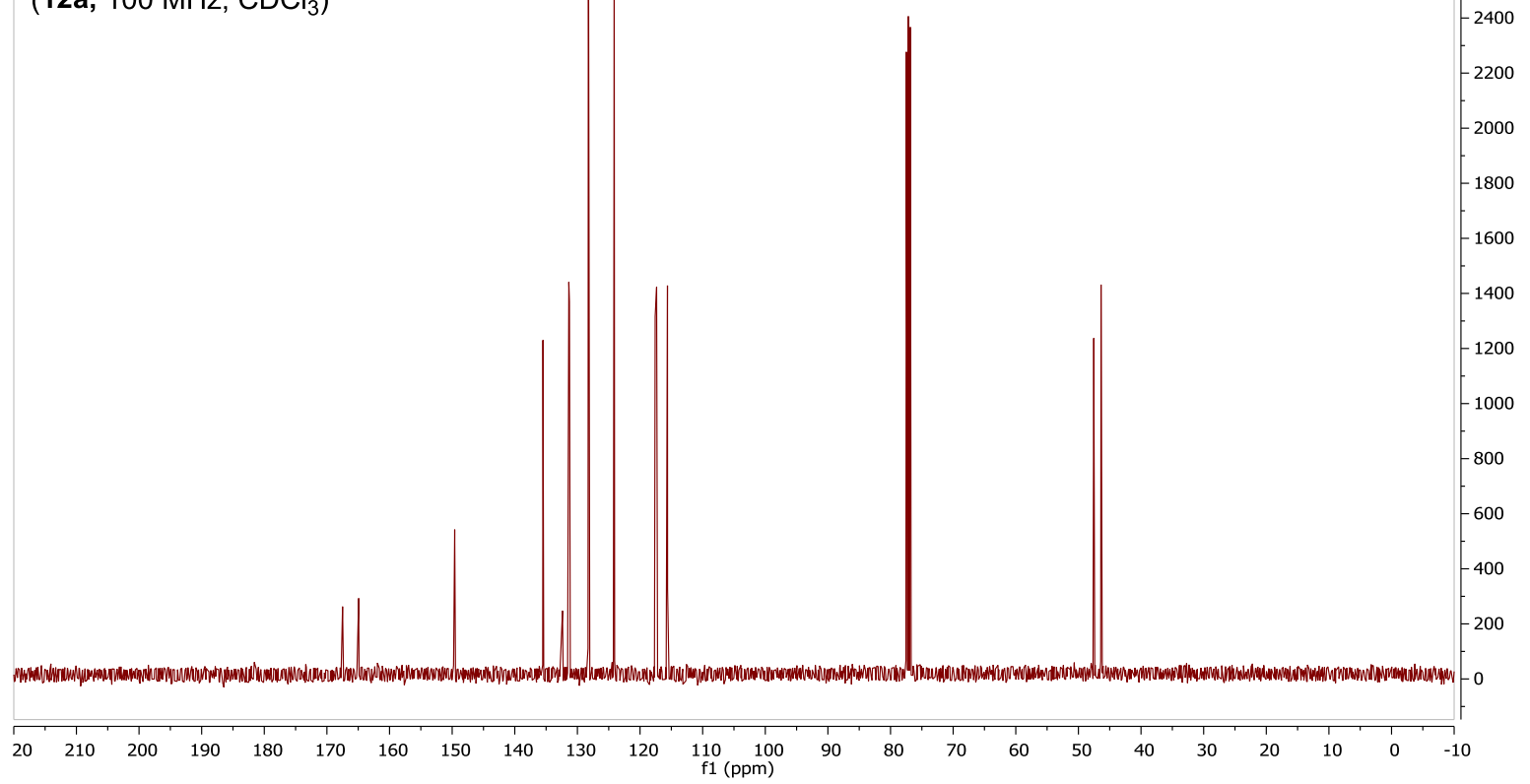




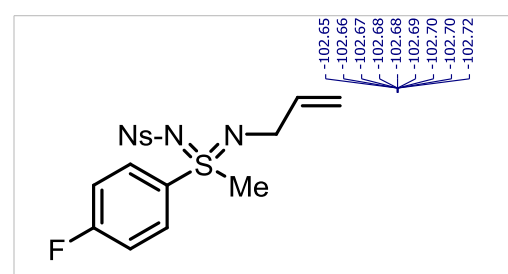

(12a, $100 \mathrm{MHz}, \mathrm{CDCl}_{3}$ )

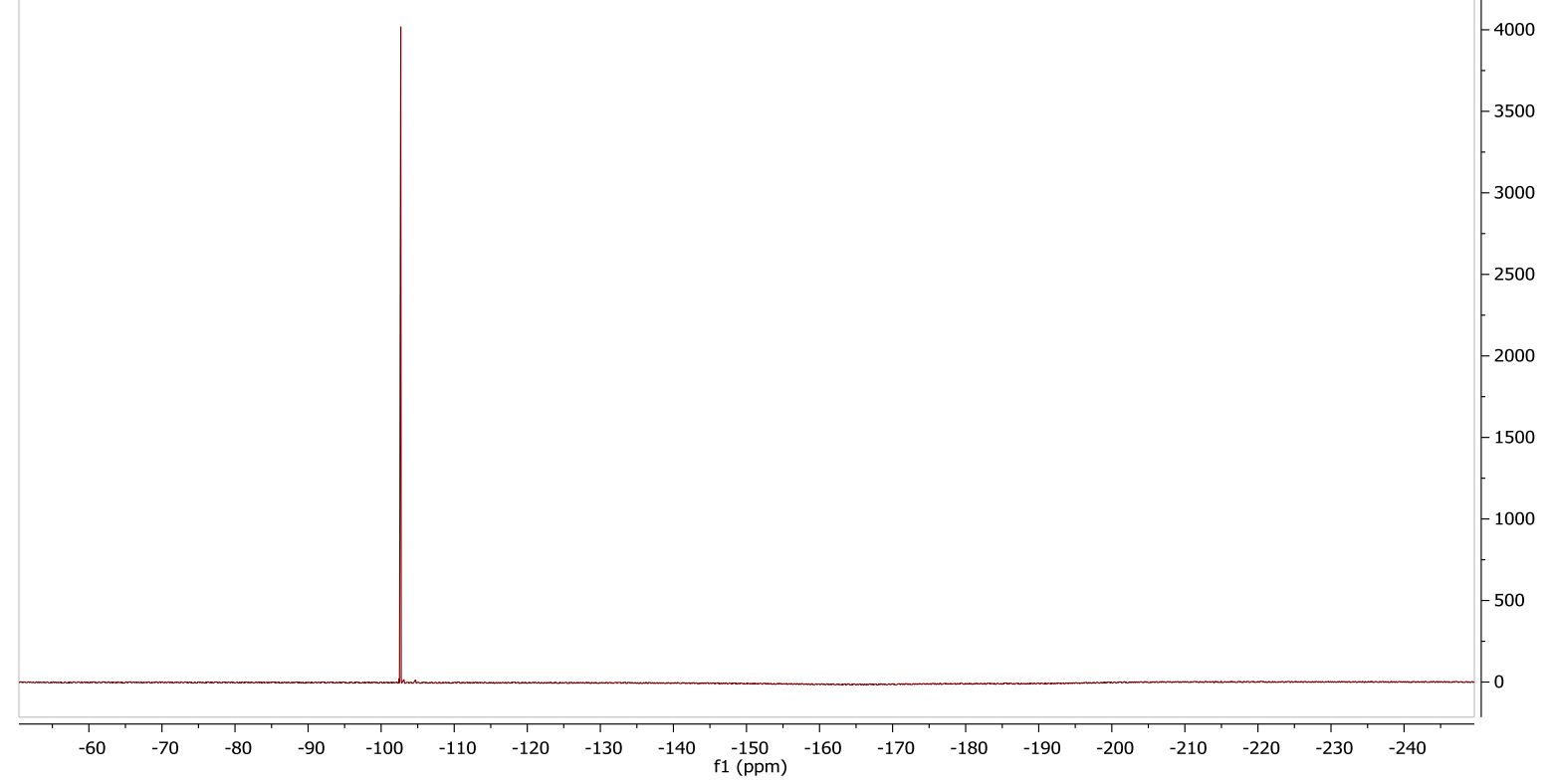

بـ<smiles>C=CCN=S(N)(=O)c1ccc(F)cc1</smiles>

$\left(12,400 \mathrm{MHz}, \mathrm{CDCl}_{3}\right)$

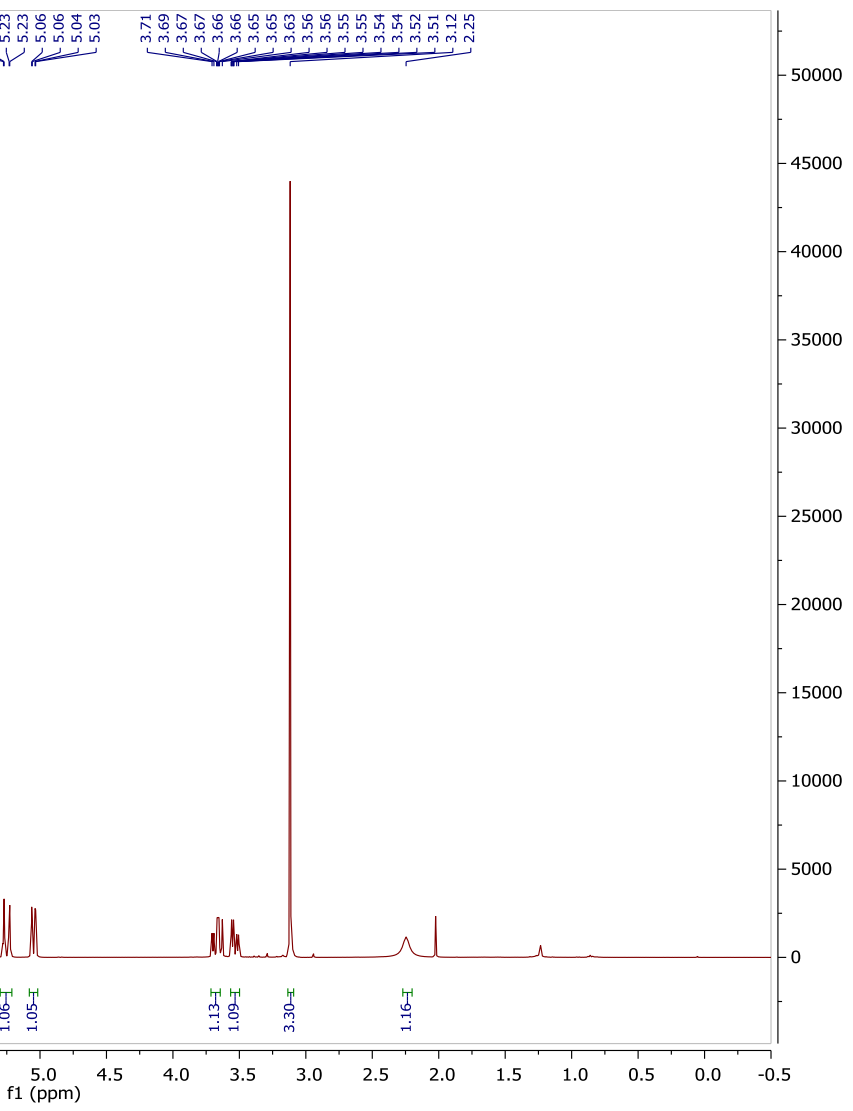



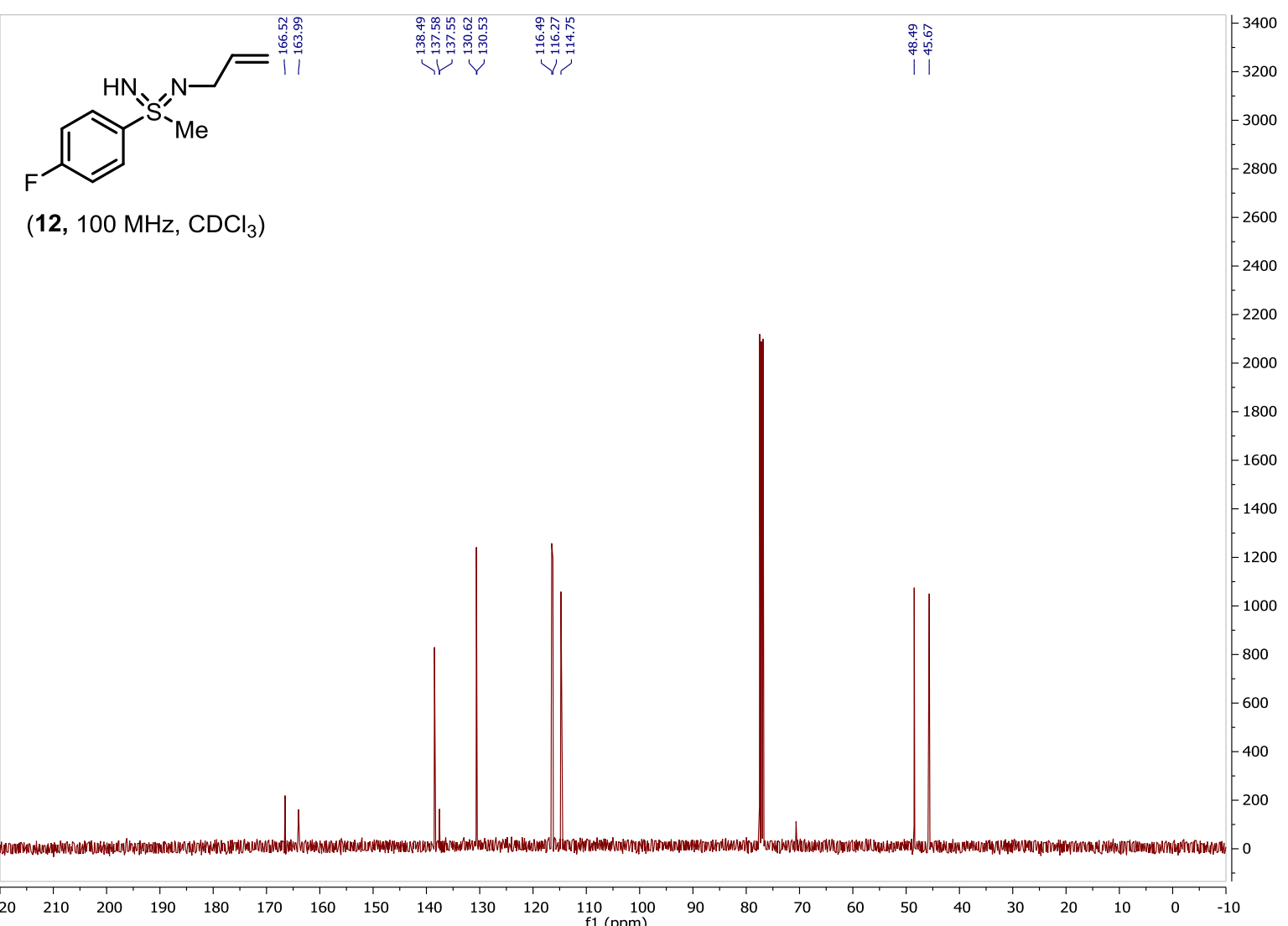

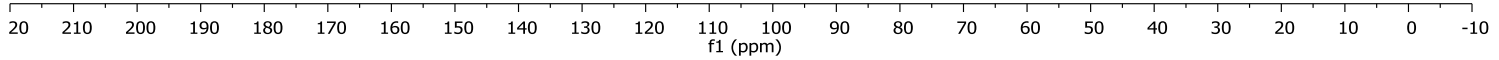

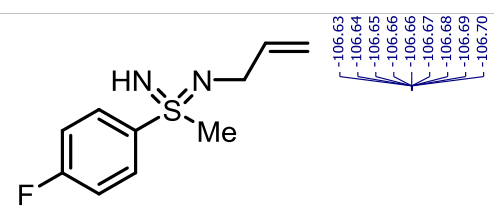

$\left(12,376 \mathrm{MHz}, \mathrm{CDCl}_{3}\right)$

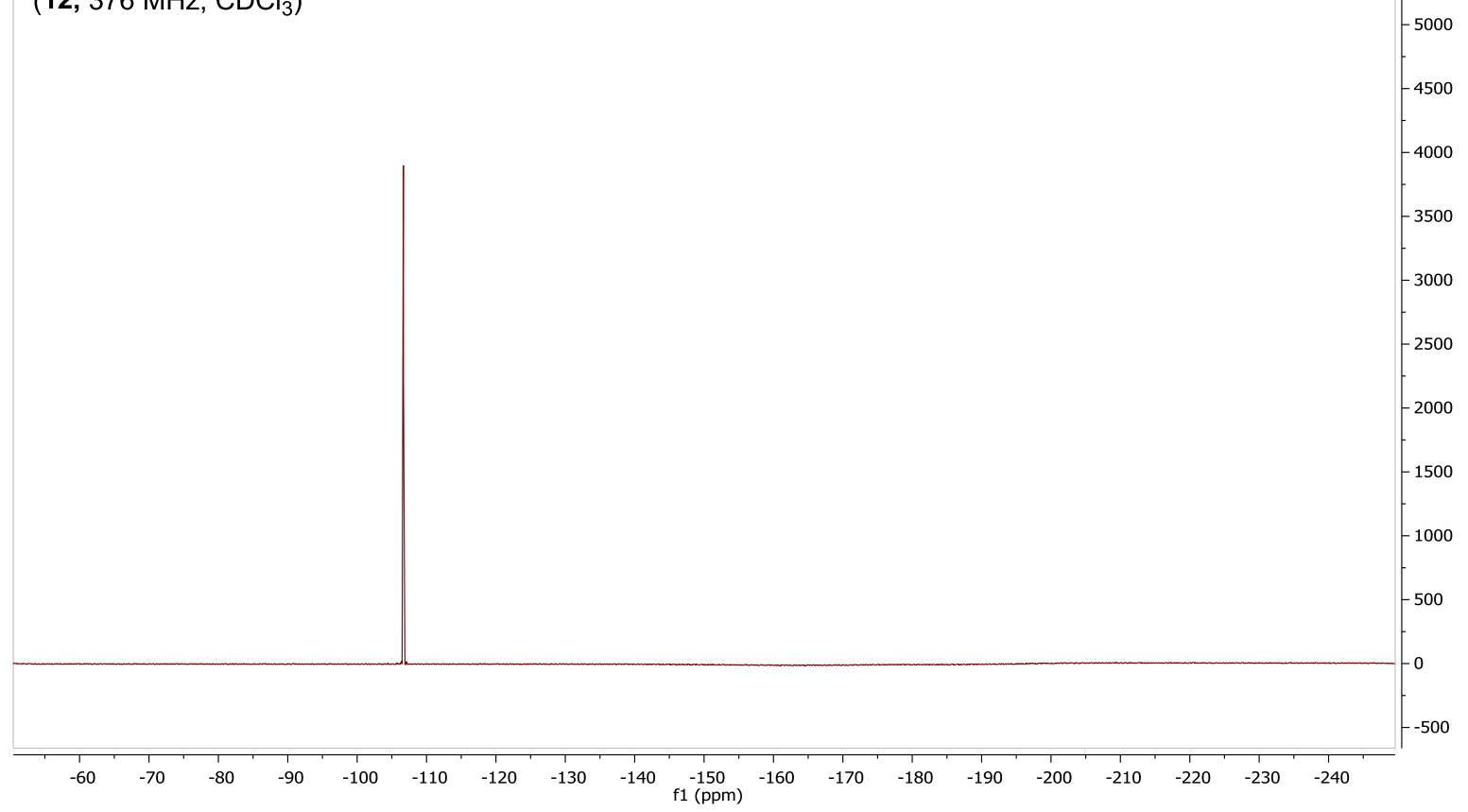




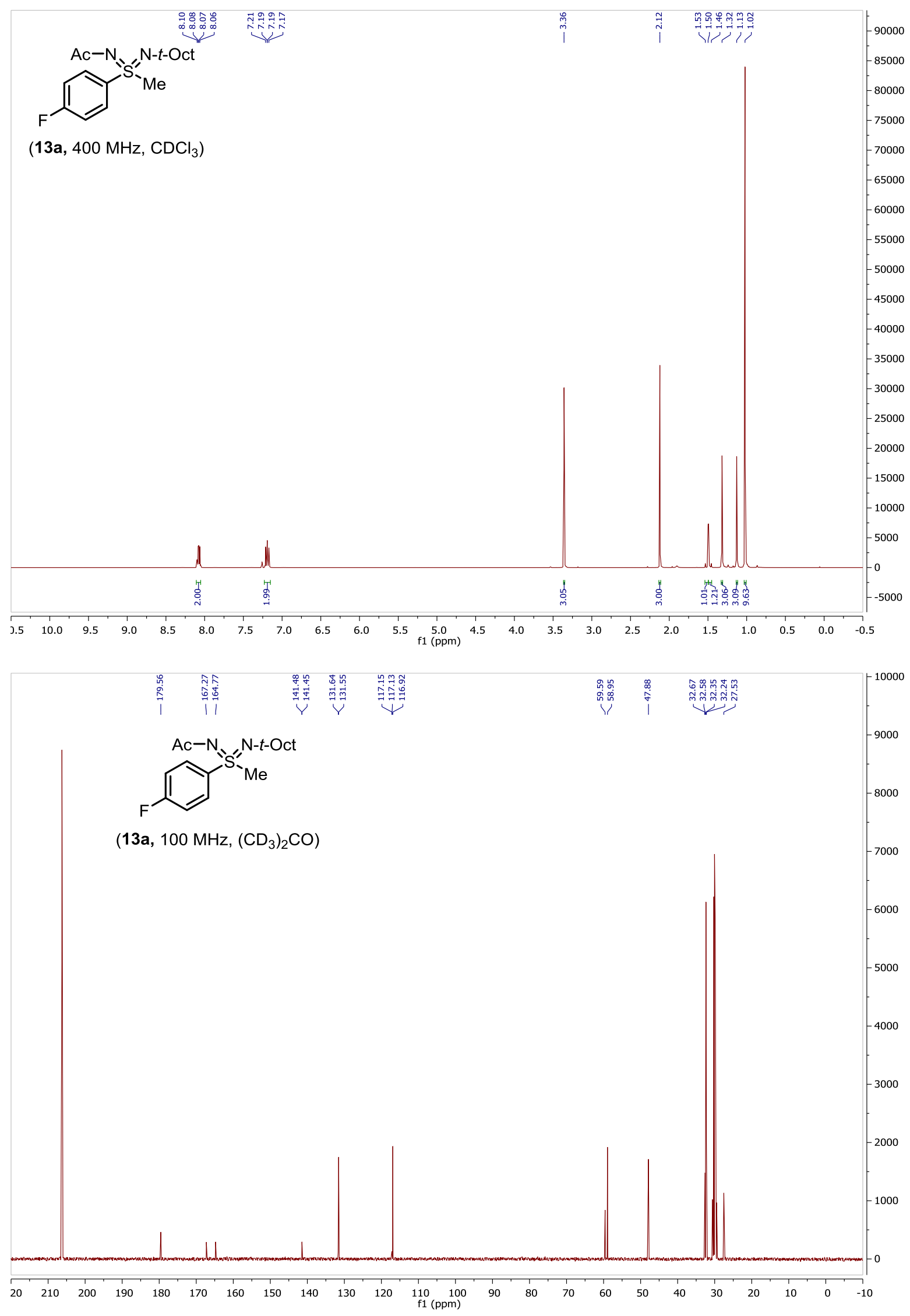



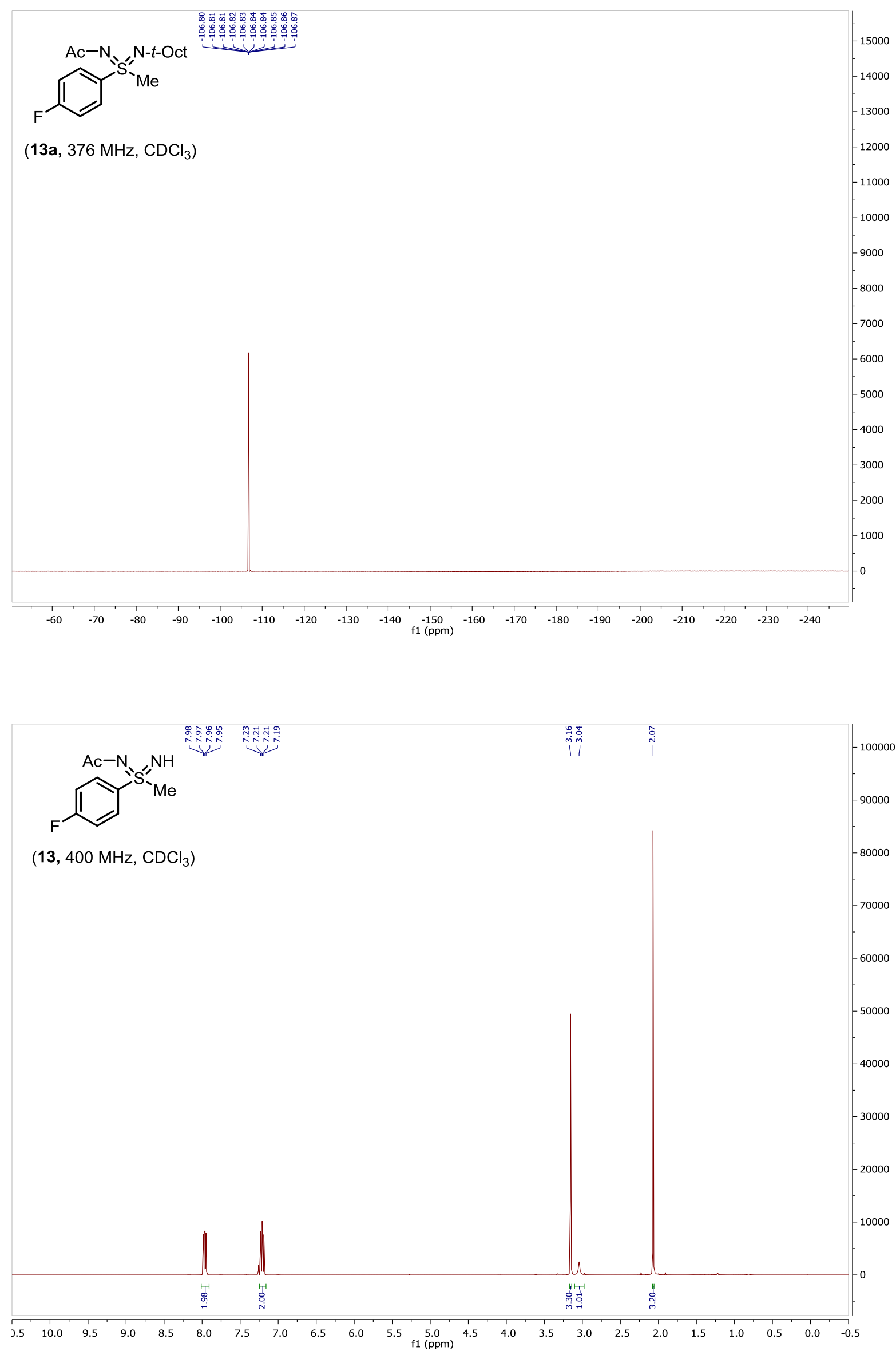


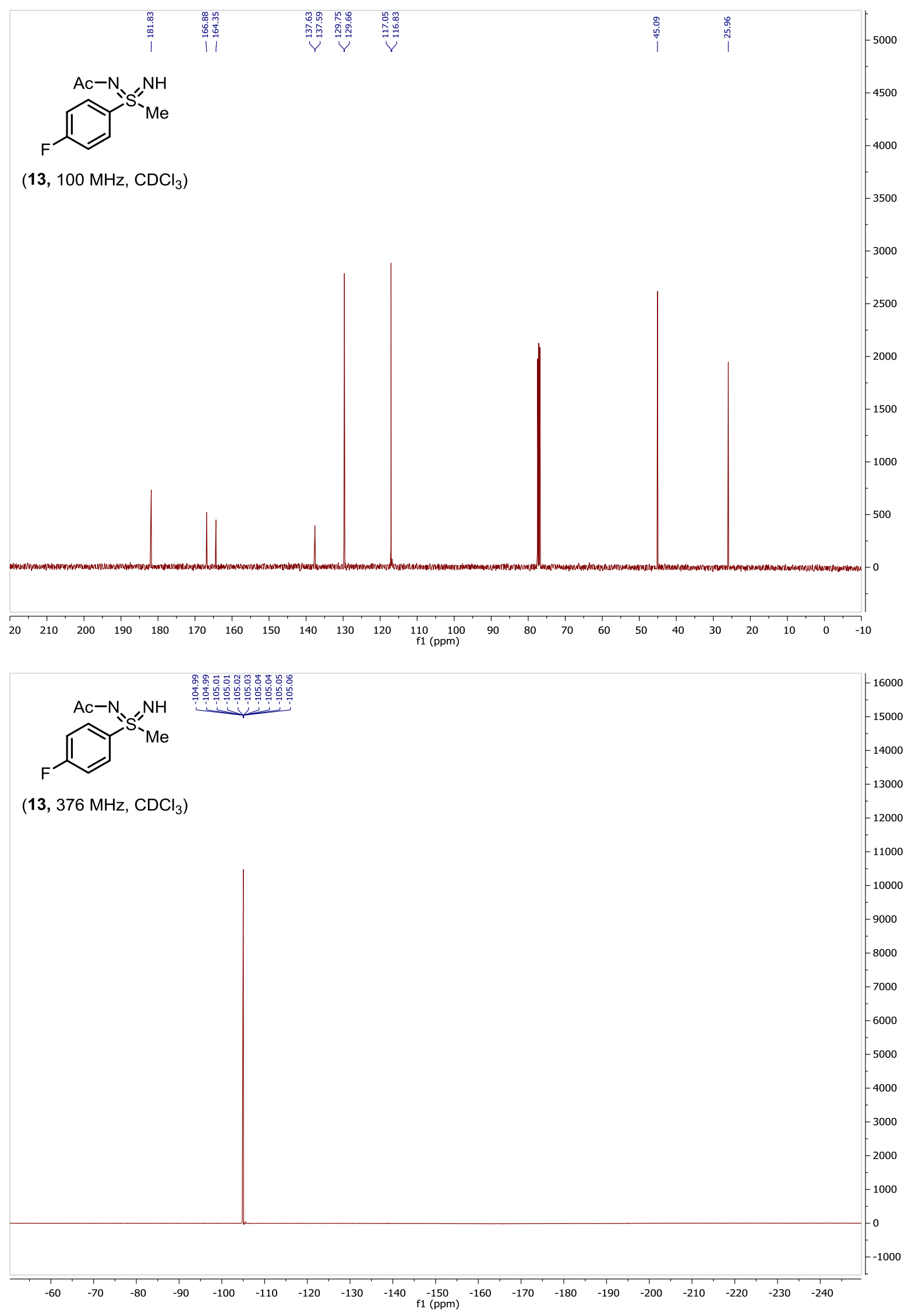



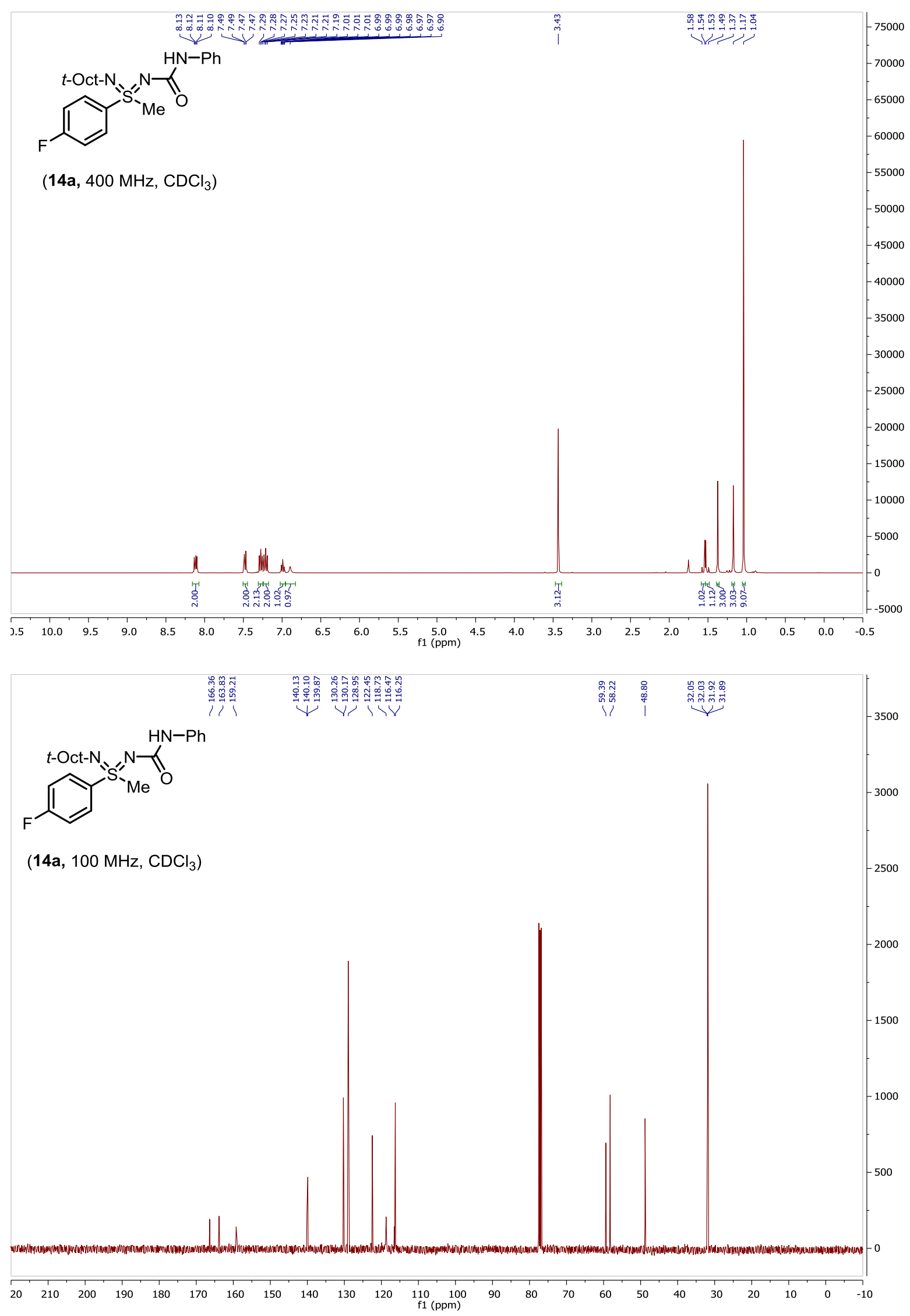

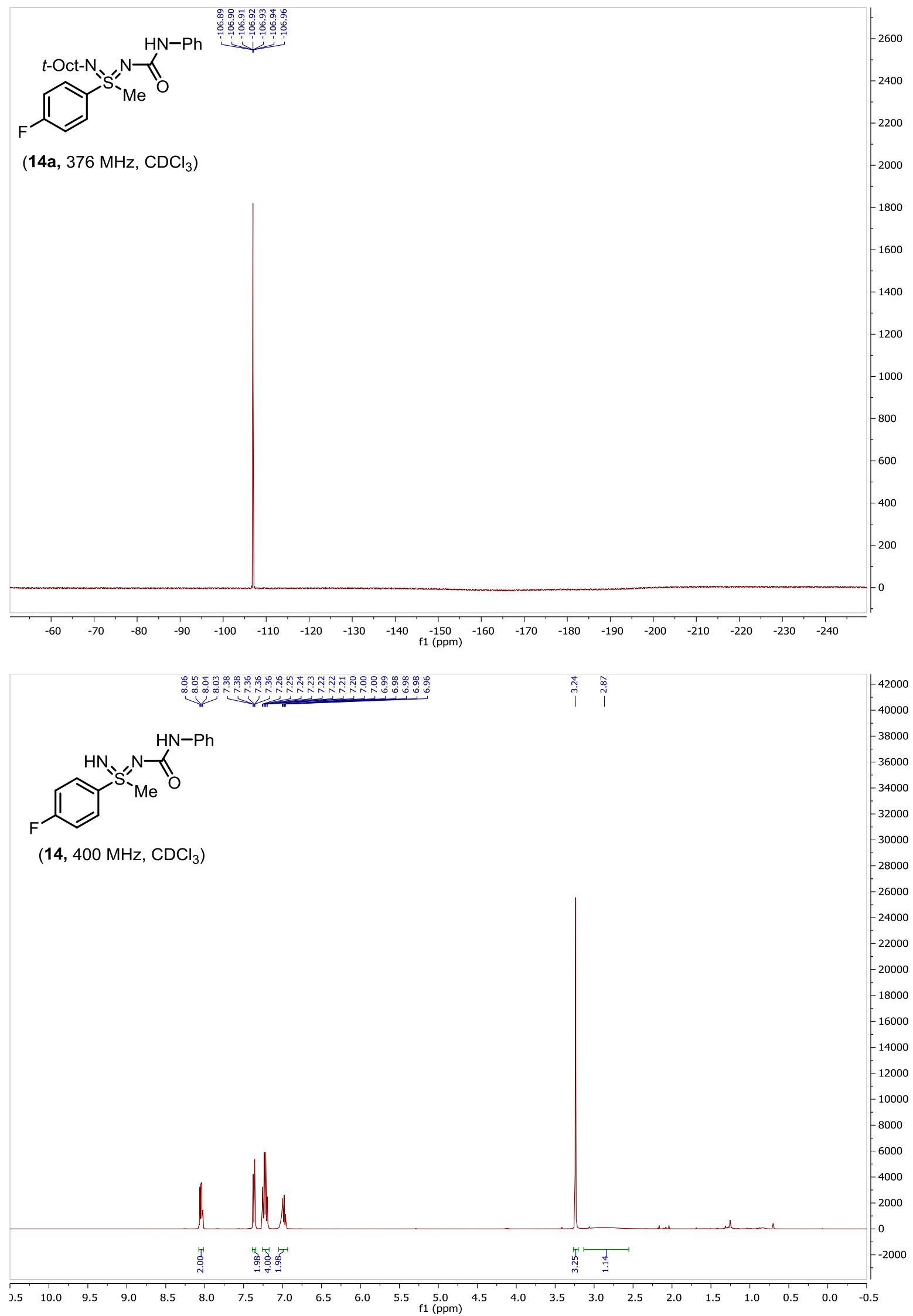


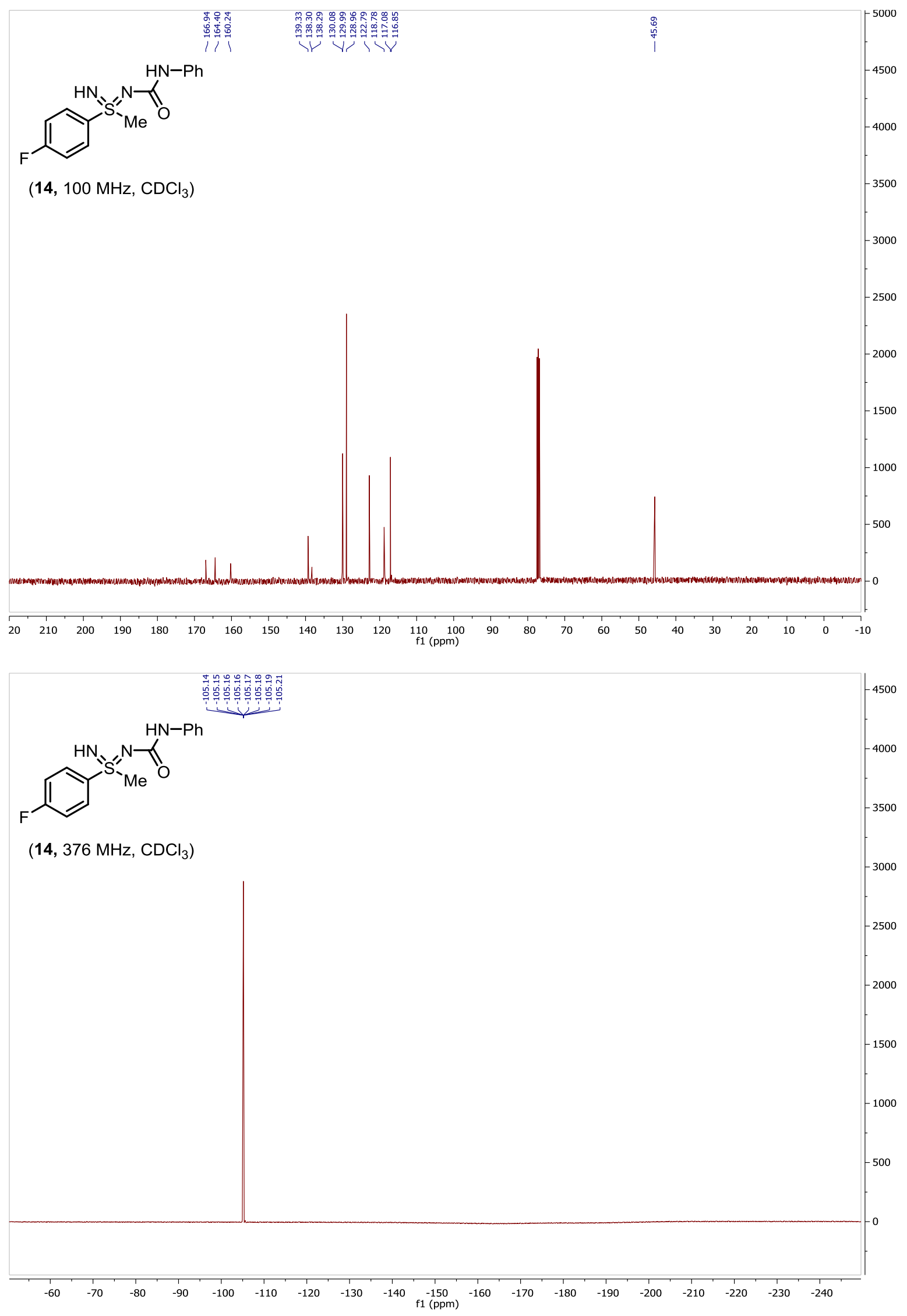



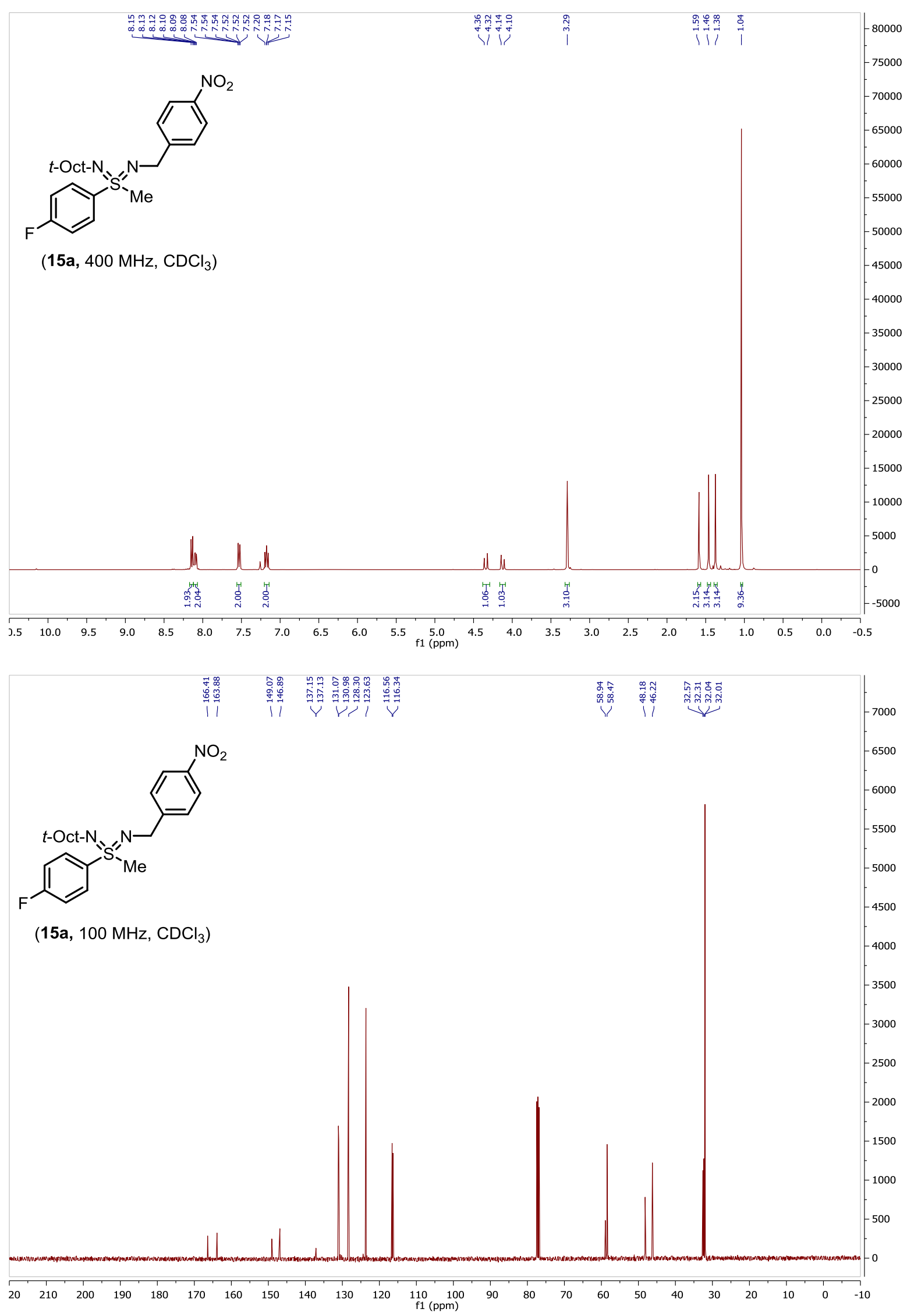

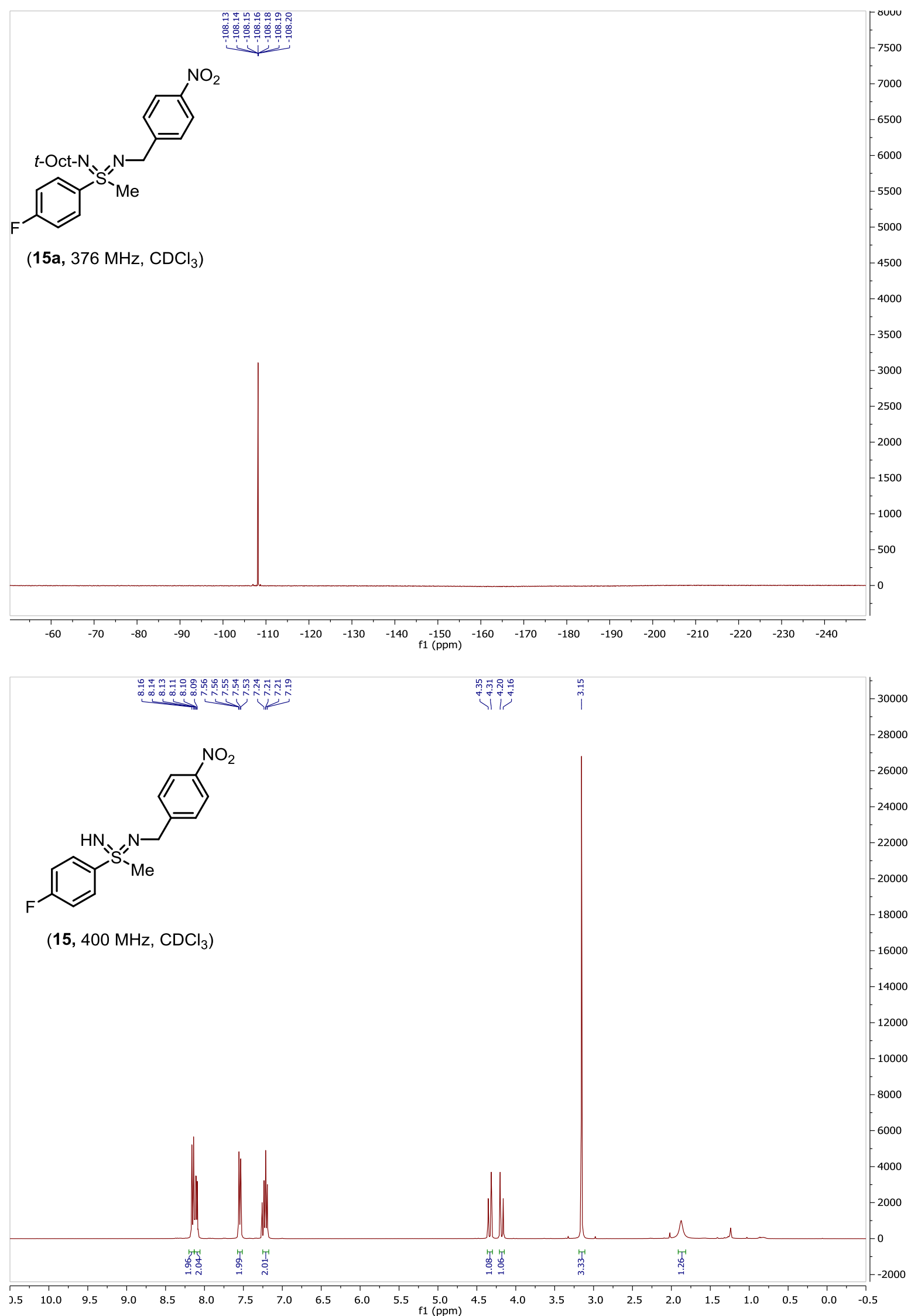

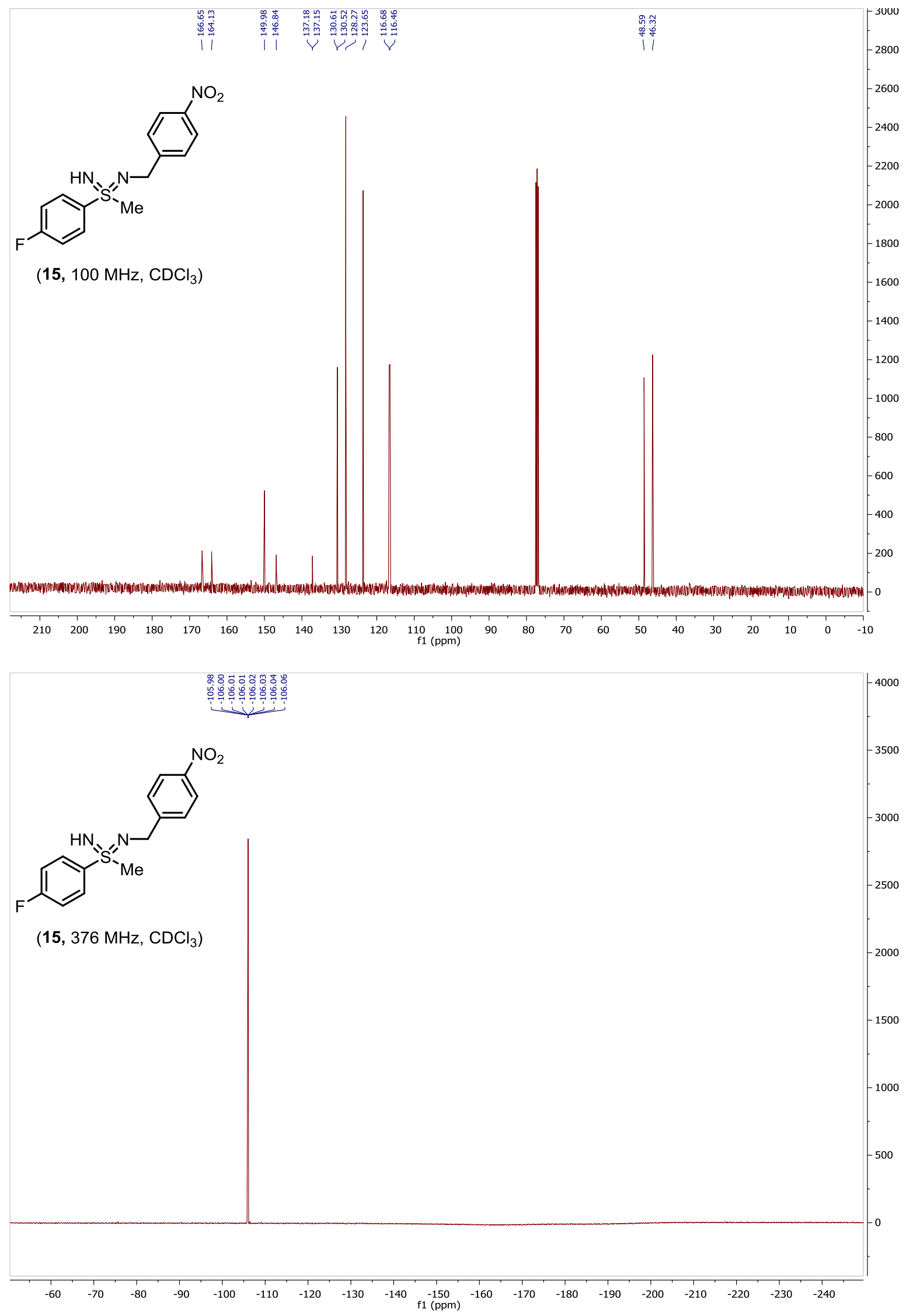


\section{References}

1 Love, B. E.; Jones, E. G. J. Org. Chem. 1999, 64, 3755.

2 Uddin, M. J.; Rao, P. N. P.; Knaus, E. E., Bioorg. Med. Chem. 2003, 11, 5273. 